\title{
THROMBOPROPHYLAXIS FOLLOWING HIP AND KNEE ARTHROPLASTY
}

\author{
Corinne Mirkazemi \\ B. Pharm Hons \\ This thesis is submitted to the University of Tasmania in fulfilment of the requirements for the \\ degree of Doctorate of Philosophy
}

University of Tasmania

School of Pharmacy

November 2013 


\section{DECLARATION OF ORIGINALITY}

This thesis contains no material which has been accepted for the award of any other degree or diploma by the university or any other institution, except by way of background information and duly acknowledged in the thesis. To the best of my knowledge and belief, this thesis contains no material previously published or written by another person except where due acknowledgement is made in the text of the thesis, nor does the thesis contain any material that infringes copyright.

Corinne Mirkazemi

November 2013 


\section{StATEMENT OF AUTHORITY OF ACCESS}

This thesis may be made available for loan and limited copying in accordance with the Copyright Act of 1968.

Corinne Mirkazemi

November 2013 


\section{STATEMENT OF CO-AUTHORSHIP}

The following people and institutions contributed to the publication of work undertaken as part of this thesis:

Corinne Mirkazemi, School of Pharmacy = Candidate

Professor Gregory Peterson, University of Tasmania = Author 1

Dr Luke Bereznicki, University of Tasmania= Author 2

\section{Author details and their roles:}

Paper 1, Thromboprophylaxis following hip and knee arthroplasty.

Located in Chapter 3: Candidate was the primary author and with author 1 and author 2 contributed to the idea, its formalisation and development.

\section{Paper 2, Are the national orthopaedic thromboprophylaxis guidelines appropriate?}

Located in Chapter 6: Candidate was the primary author and with author 1 and author 2 contributed to the idea, its formalisation and development.

\section{Paper 3, Response to Re: Are the national orthopaedic thromboprophylaxis guidelines} appropriate?

Located in Chapter 6: Candidate was the primary author and with author 1 and author 2 contributed to the idea, its formalisation and development.

We the undersigned agree with the above stated "proportion of work undertaken" for each of the above published (or submitted) peer-reviewed manuscripts contributing to this thesis:

Signed:

(Professor G. Peterson)

Supervisor

School Of Pharmacy

University of Tasmania

(Dr. Luke Bereznicki)

Head of School

School of Pharmacy

University of Tasmania

Date: 


\section{ACKNOWLEDGEMENTS}

I have been very fortunate to be guided and assisted by many experienced and supportive individuals in conducting my research. Professor Gregory Peterson, Dr Luke Bereznicki and Dr Emily Hansen have together provided the necessary leadership and guidance to support me throughout my candidature, and I thank each of them.

I am indebted to Professor Peterson for encouraging me to embark on my candidature; you've always going above and beyond what was necessary, to ensure that everything was in place personally and professionally to enable me to complete it. You were a great honours supervisor, and an even better PhD supervisor - I sincerely thank you.

Thank you to Dr Bereznicki for your constant encouragement, your eye for detail and your great sense of humour, which always uplifted the atmosphere of our meetings. You are a trailblazer for our generation of pharmacists and I appreciate your careful mentorship over these years.

I would like to express my gratitude to Dr Hansen for teaching me how to look past the numbers and find meaning in my data. Your positive attitude always left me feeling better after our meetings - a greatly appreciated and invaluable trait in a supervisor.

I would like to thank the University of Tasmania School of Pharmacy and the Royal Hobart Hospital Research Fund for providing me with the financial means to complete the projects.

And finally, my family: Thank you to my grandparents Dr Heshmat Ta'eed, Mrs Nosrat Ta'eed, Mr David Garnett and Mrs Pat Garnett, for laying the firm foundation on which I now stand. Without you, I am nothing, and I dedicate any small measure of success I have to you.

I am forever grateful to my parents Mr Fuad Ta'eed and Dr Gillian Ta'eed who have lent me their support over the years in many countless ways. Thank you to my brothers Dr Vahid Ta'eed, Mr Collis Ta'eed and Dr Anis Ta'eed for their love. I am so proud of each and every one of you and hope that I too can make you proud.

And last but not least, thank you to my husband Mr Arman Mirkazemi. This PhD was your idea, and you subsequently endured the long process with me, always offering your assistance, support, encouragement, love and your unique sense of humour. You've always believed in me, and inspired me to strive for more. Without you, none of this would have been possible. 


\section{CONTENTS}

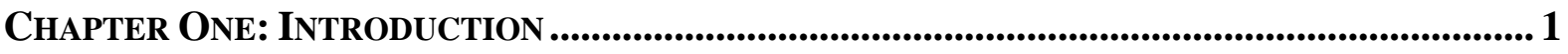

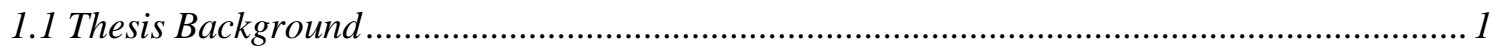

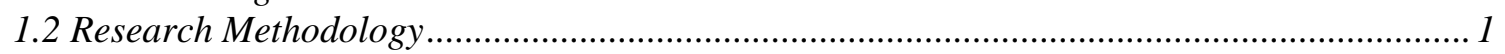

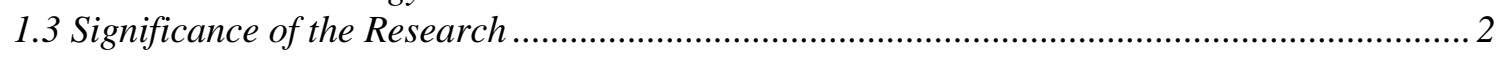

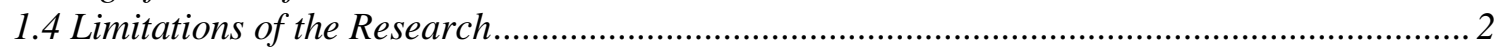

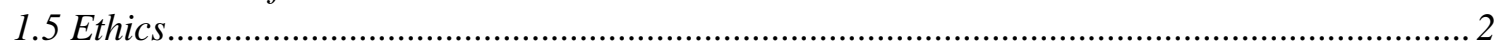

ChaPTER TWO: THROMbOPROPHYLAXIS FOLlOWING HIP AND KNEE ARTHROPLASTY ..... 3

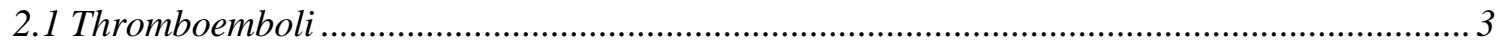

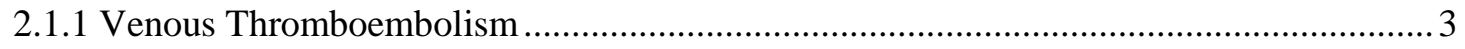

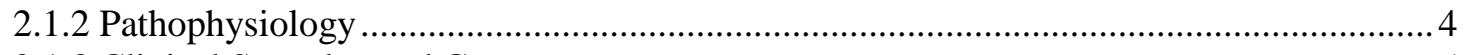

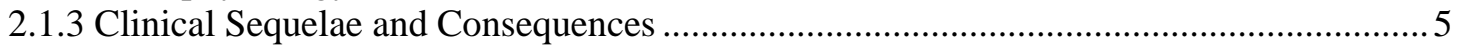

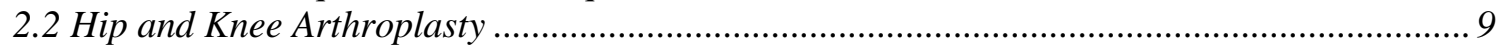

2.2.1 Postoperative Venous Thromboemboli ………............................................................ 9

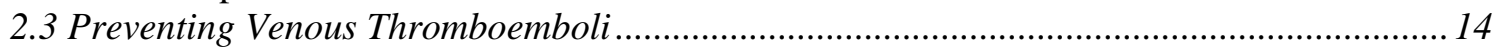

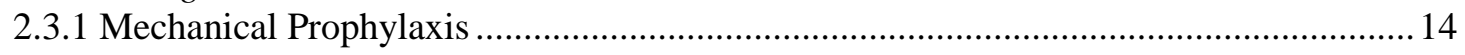

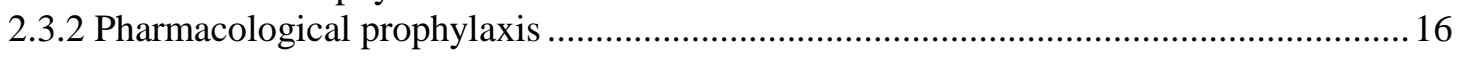

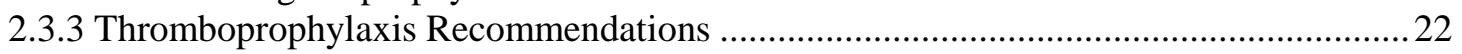

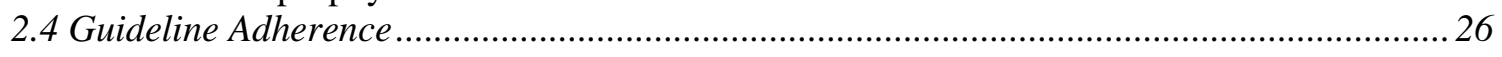

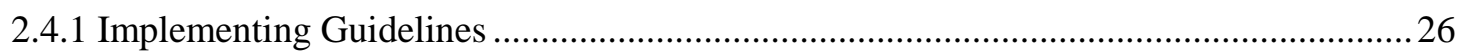

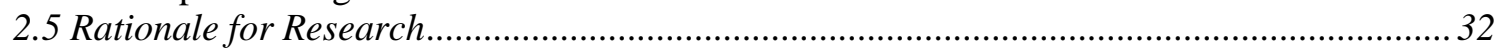

Chapter Three: Local Thromboprophylaxis Prescribing ...................................33

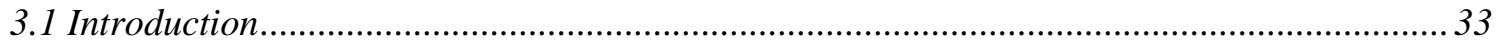

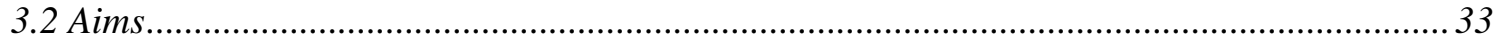

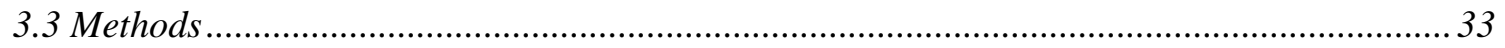

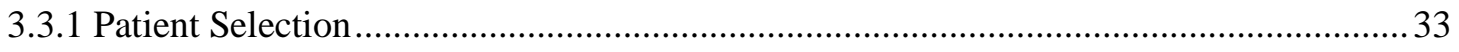

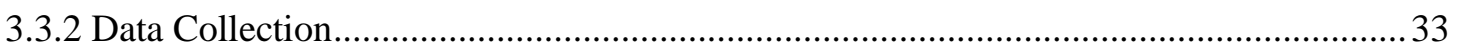

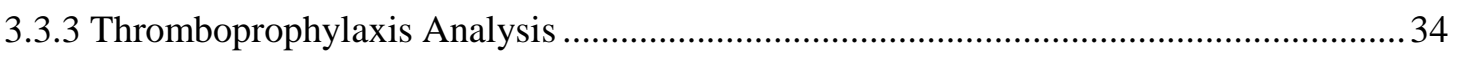

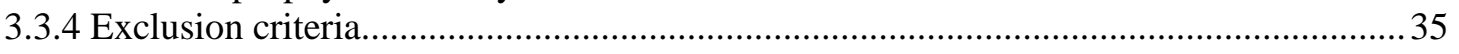

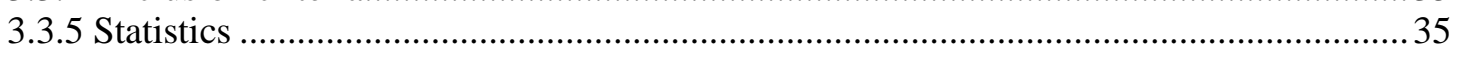

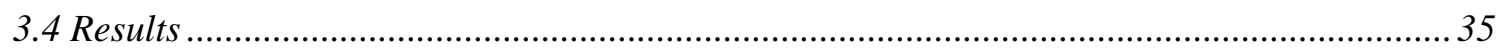

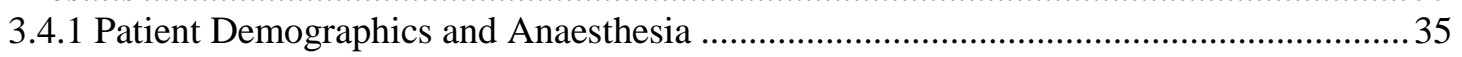

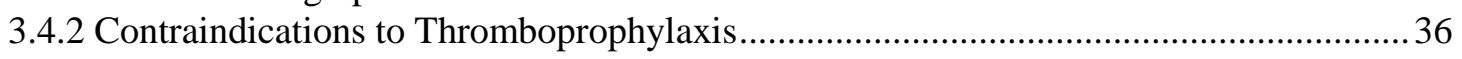

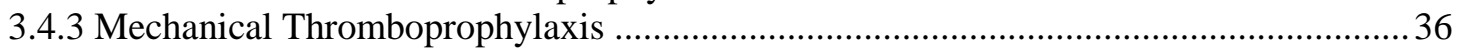

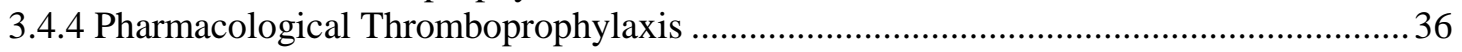

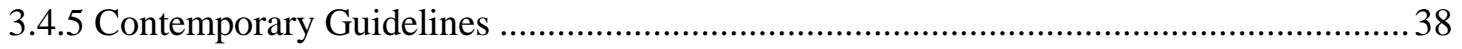

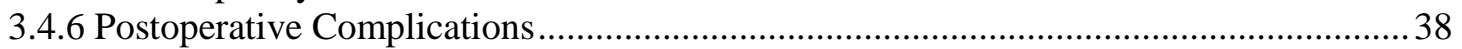

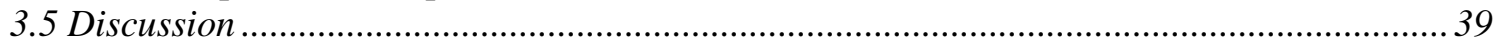

Chapter Four: Prescribing in Australian Public Hospitals...................................... 42

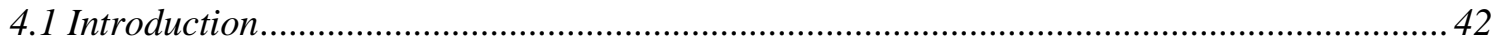

4.2 Aim

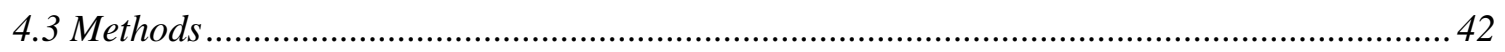

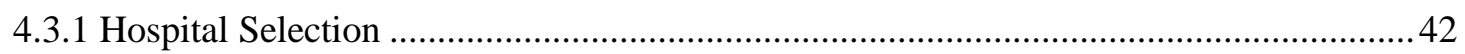

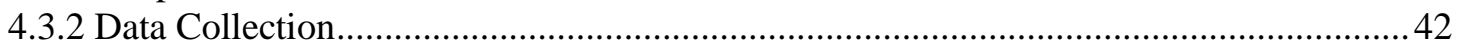

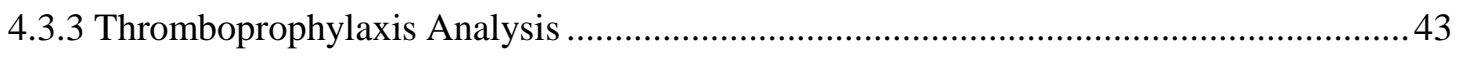

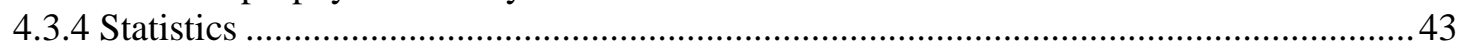

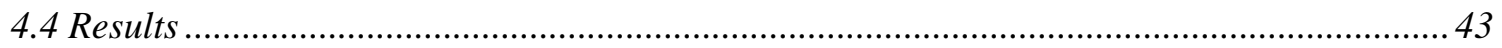




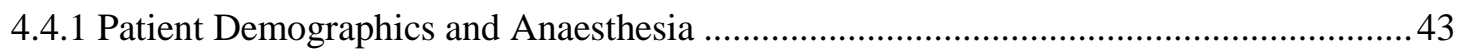

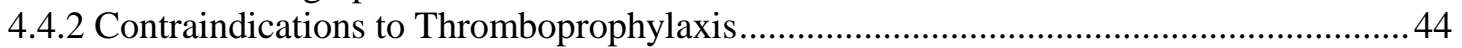

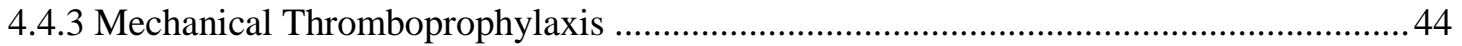

4.4.4 Pharmacological Thromboprophylaxis ....................................................................... 44

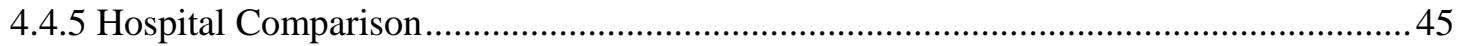

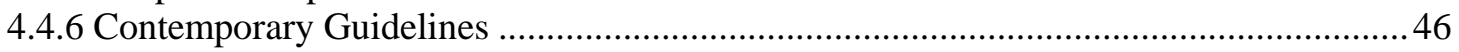

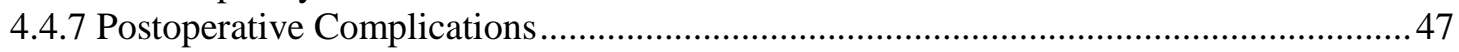

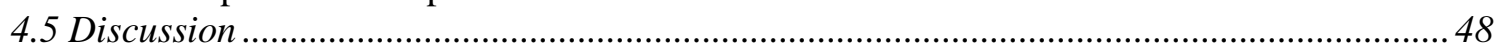

Chapter five: Australian Hospital Protocols......................................................50

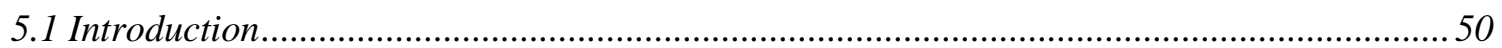

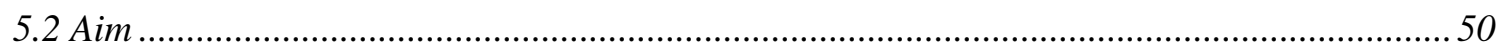

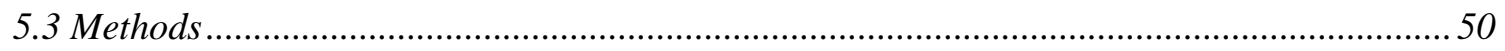

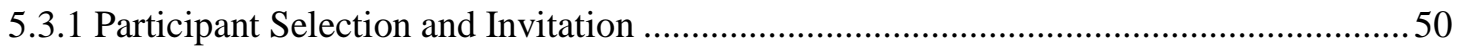

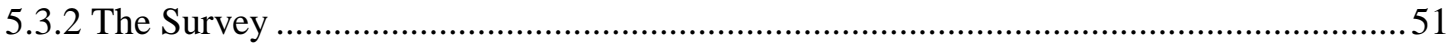

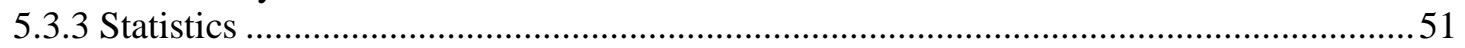

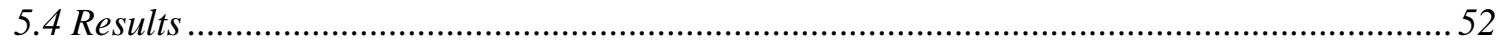

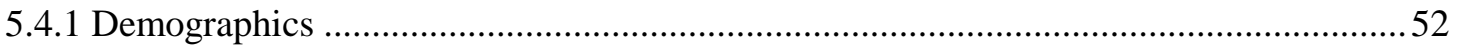

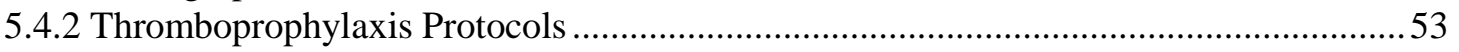

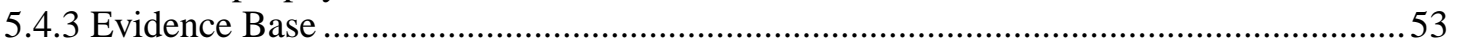

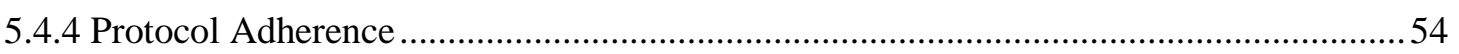

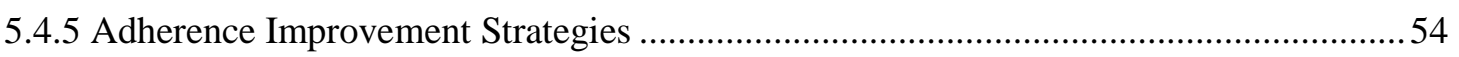

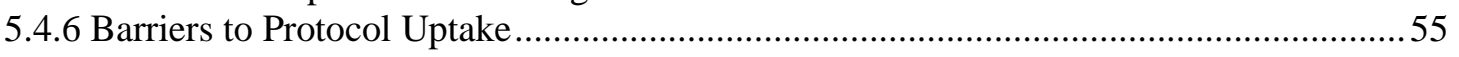

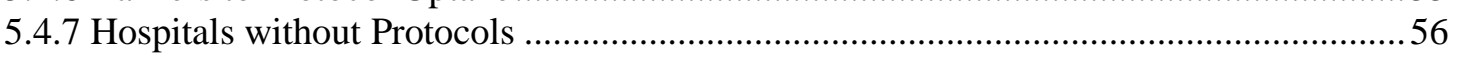

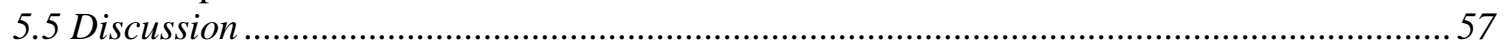

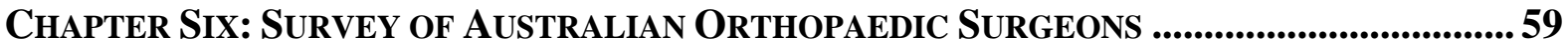

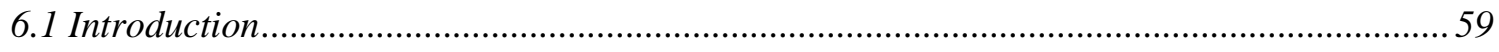

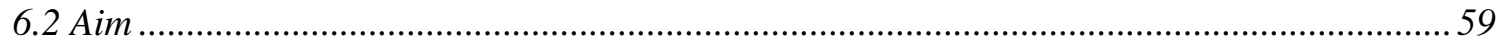

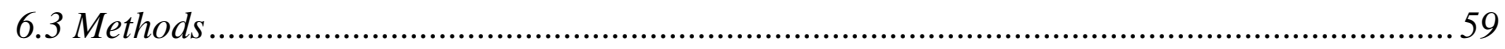

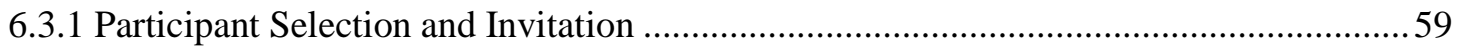

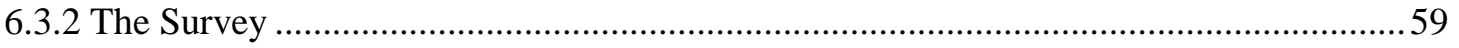

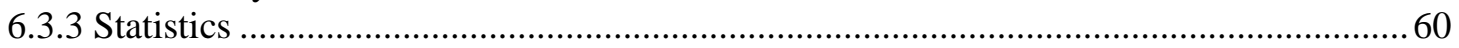

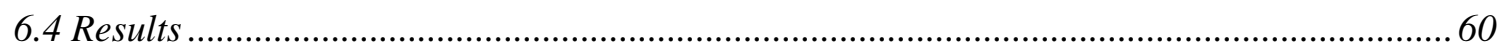

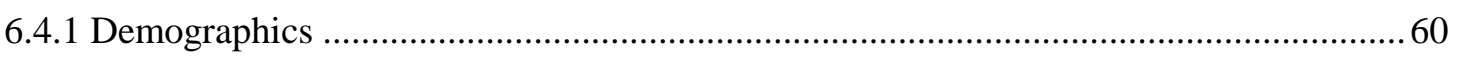

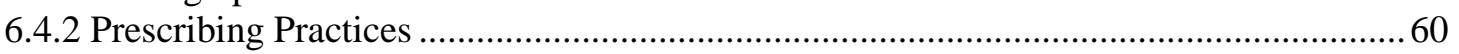

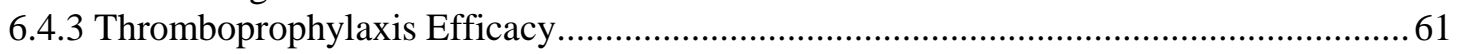

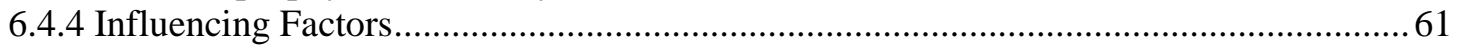

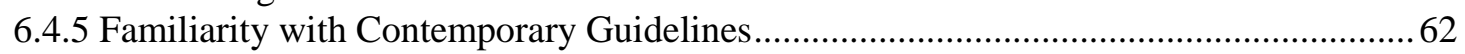

6.4.6 Case Studies and Thromboprophylaxis Prescribing...................................................63

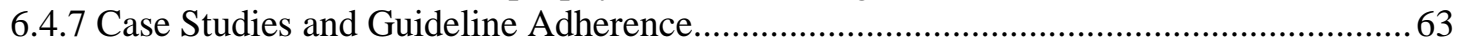

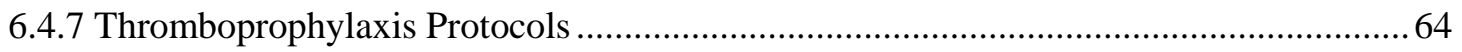

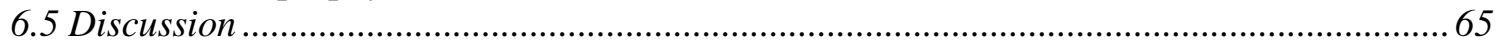

Chapter Seven: Health Professionals' Perceptions ......................................................... 68

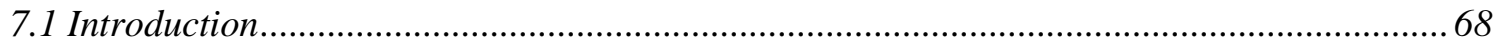

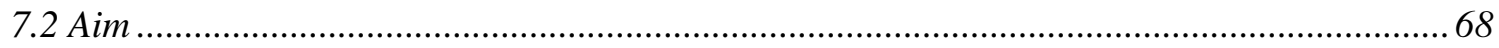

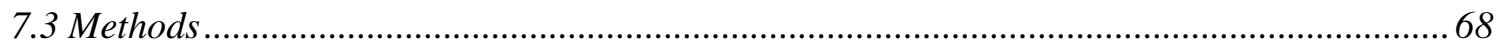

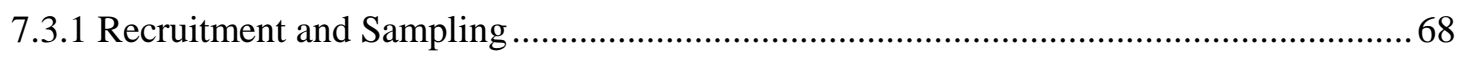

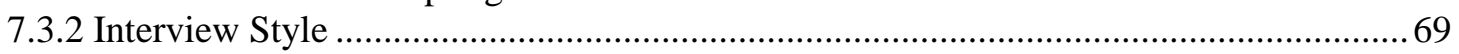

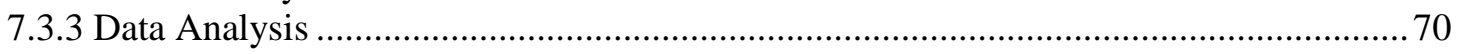

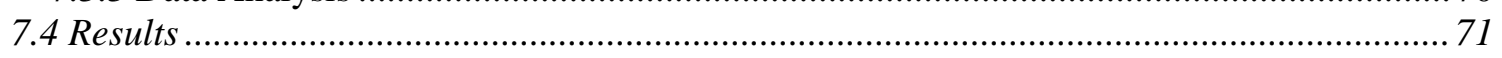

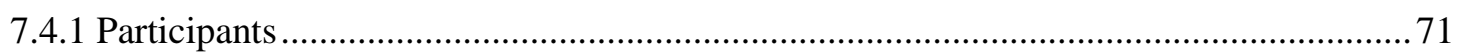

7.4.2 Surgical and Postoperative Changes ............................................................................ 71

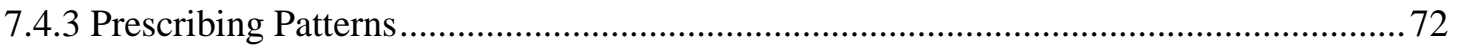




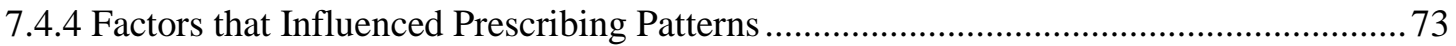

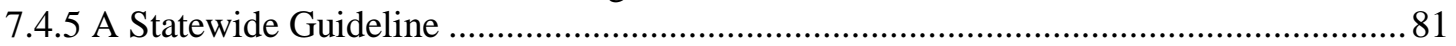

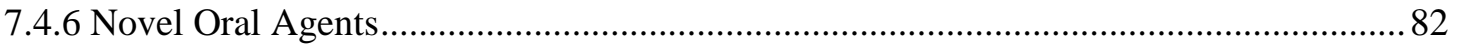

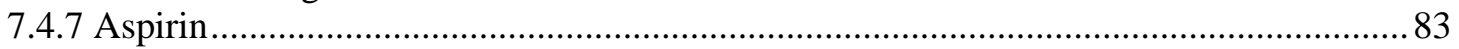

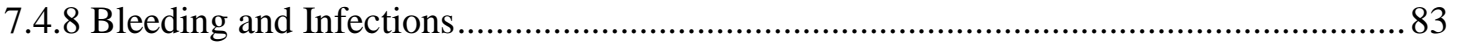

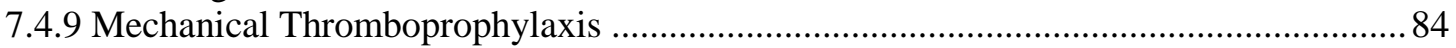

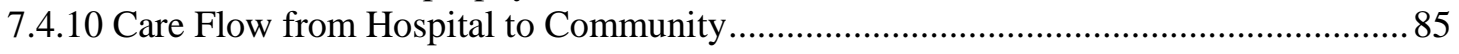

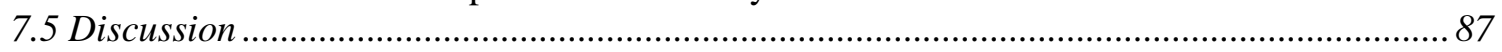

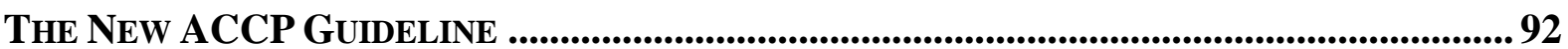

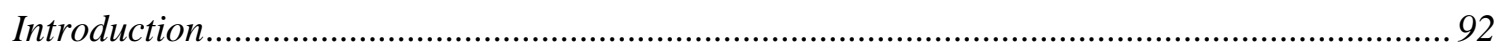

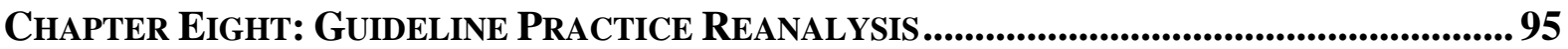

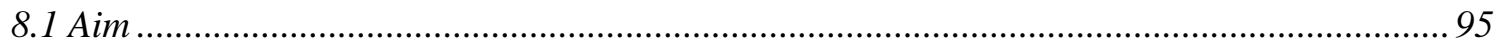

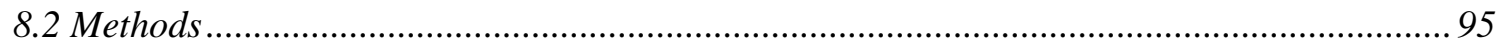

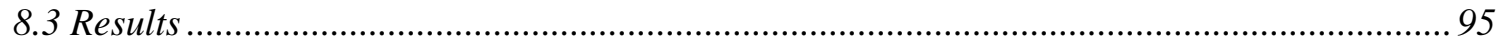

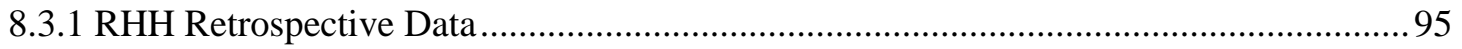

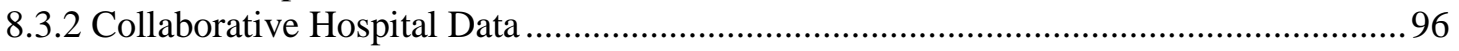

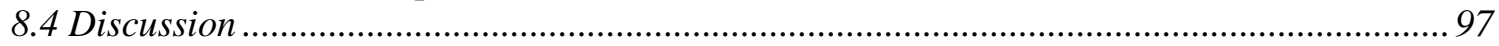

ChaPTER Nine: SURgeONS AND THE ACCP 9TH ED. GUIDELINE .....................................98

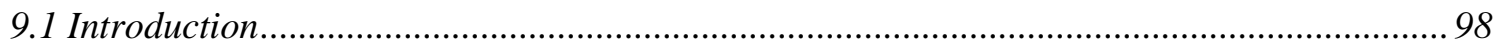

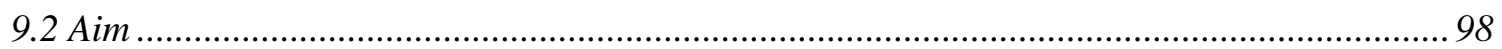

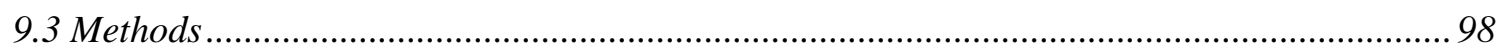

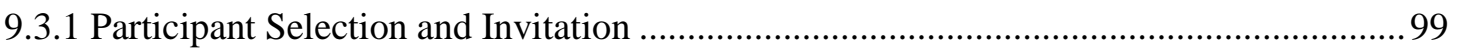

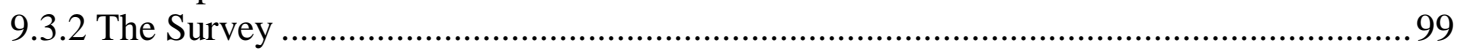

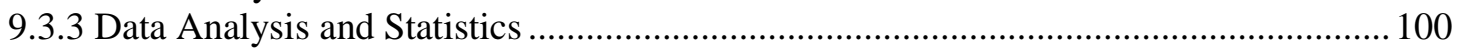

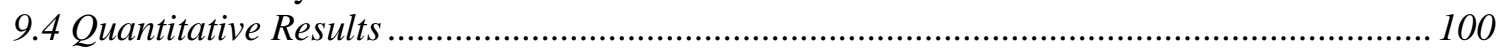

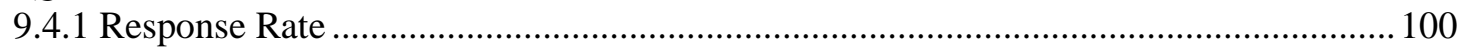

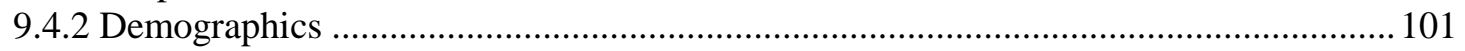

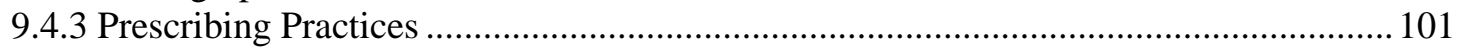

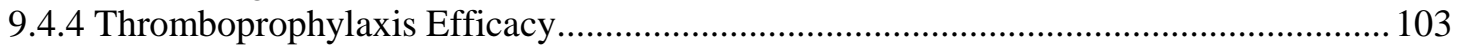

9.4.5 Factors that Influence Thromboprophylaxis Prescribing ............................................. 103

9.4.6 Familiarity with Contemporary Guidelines................................................................... 105

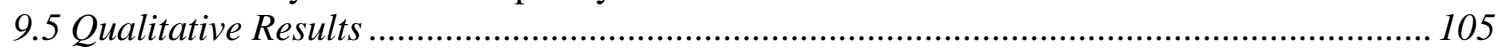

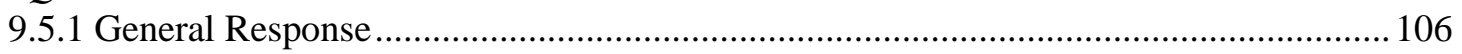

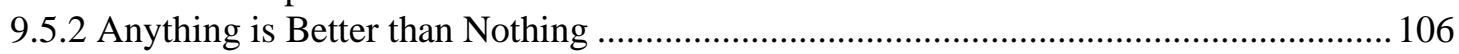

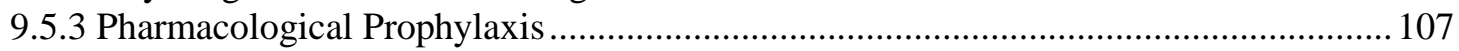

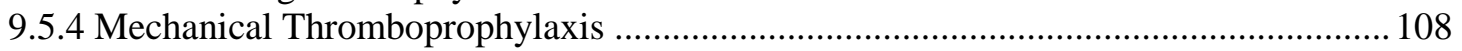

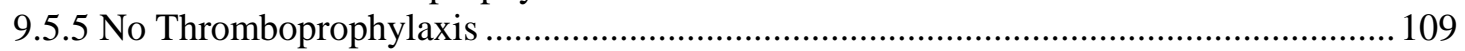

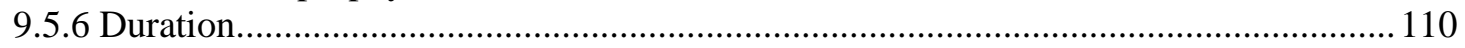

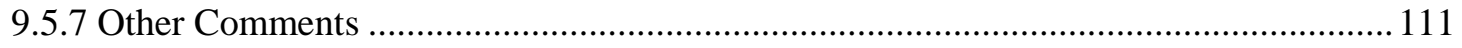

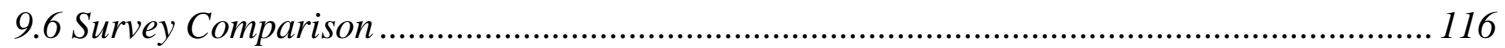

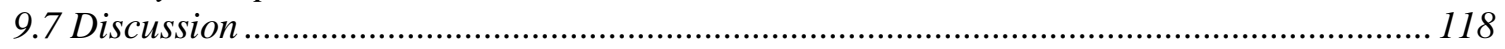

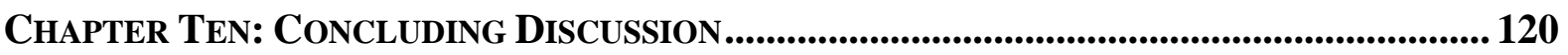

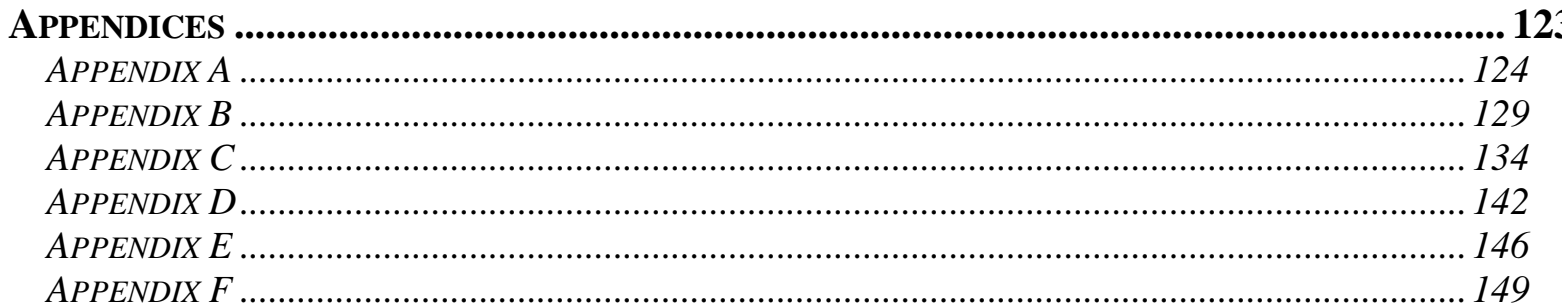




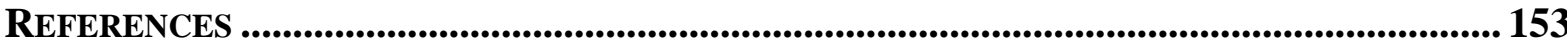




\section{AbSTRACT}

Hip and knee arthroplasty are increasingly common surgical procedures in Australia, with more than 85,000 expected to be performed this year. Compared to the general public, people who undergo a hip or knee arthroplasty are at an increased risk of developing blood clots (also known as a venous thromboemboli or VTE) as both procedures activate all three aspects of Virchow's risk triad during the pre-, peri- and postoperative period.

Importantly, VTE are considered one of the most common causes of preventable death in Australian hospitals. Whilst there are mechanical and pharmacological measures that can be employed to prevent VTE (known collectively as thromboprophylaxis), and many national and international bodies have released thromboprophylaxis guidelines over the years, previous studies have reported consistent underutilisation of thromboprophylaxis both in Australia and overseas. The overarching objective of this thesis was to examine thromboprophylaxis prescribing patterns in Australia, and the factors that influence them.

To achieve this aim, a number of complementary studies were conducted, including both qualitative and quantitative work. Retrospective reviews were conducted at six hospitals across three states to explore prescribing patterns; the results indicated that while anticoagulant thromboprophylaxis is routinely used in hospital, its continuation at discharge is not habitual despite recommendations by contemporary guidelines to extend therapy usually beyond the inpatient period. Interestingly, prescribing patterns were generally found to be more aligned with recent contemporary recommendations than recommendations existent at the time of prescribing, indicating practice preceded guidelines.

A survey exploring hospital protocols and local prescribing practices identified that only $50 \%$ of the 143 Australian hospitals surveyed had thromboprophylaxis protocols and that their alignment with published literature varied considerably. Surprisingly, private hospitals tended to base their guidelines on the National Health and Medical Research Council (NHMRC) Guideline, whereas public hospitals were more likely to favour individual or collective surgeon preferences. Where they existed, adherence to hospital protocols was apparently high (86\%), and predictably trended to increase in hospitals with adherence strategies in place (91\% vs. $79 \%$ ).

Two online surveys were conducted with orthopaedic surgeons practising in Australia. Whilst the first survey was limited by its small number of respondents (25), it identified that individual prescribing preferences vary significantly, with many surgeons perceiving guideline recommendations (including those of the NHMRC Guideline) as inappropriate. Although few respondents believed that pharmacological prophylaxis prevents fatal pulmonary emboli, and many 
responded that VTE risk is low following arthroplasty, all participants routinely prescribed pharmacological prophylaxis postoperatively. In addition to bleeding concerns and a perceived low VTE risk, the third strongest barrier to thromboprophylaxis use was the belief that study evidence is not applicable to real-world inpatient populations. Along this line, a number of surgeons expressed suspicions that industry funding was heavily biasing guideline recommendations, either directly by sponsoring guideline authors or indirectly by sponsoring associated research. The second survey had a significantly larger number of respondents (221), and maintained the results of the first study.

Semi-structured interviews were used to explore surgeons' preferences, opinions and experiences in greater detail. Moreover, to complement these views and to gain a better understanding of real practice, these interviews were supplemented by interviews with Tasmanian based orthopaedic interns, general practitioners (GPs) and clinical pharmacists. While a surgeon's opinion was identified to be greatly moulded by their training and collective experience, and not by contemporary guidelines, other health professionals' opinion were more inclined to be shaped by guidelines. The interviews also identified that even though participants differed in their thromboprophylaxis preferences and opinions, they all strongly agreed that thromboprophylaxis should be tailored to the individual patient. All surgeons expressed familiarity with at least one contemporary guideline; however, other health professionals generally did not. This lack of knowledge and familiarity naturally impairs their ability to contribute to postoperative care. Additionally, hospital to community communication was described as slow and unreliable by participants, further disadvantaging GPs from being able to effectively contribute to VTE prevention after discharge.

Rather than awaiting the formulation of a Tasmanian based protocol (which was attempted by an outside group early on in the research), another strategy to optimise thromboprophylaxis use would be to instigate a statewide strategy to increase the uptake of the NHMRC Guideline. One of the biggest barriers identified in this research to the adoption of guidelines such as the NHMRC Guideline is the perception that they impose a 'one size fits all' approach to thromboprophylaxis by making 'carte blanche recommendations'. In reality, the NHMRC Guideline takes a comprehensive approach to VTE prophylaxis and, unbeknown to many health professionals, actually addresses the many concerns they expressed in the studies in this thesis. A quick readyreference tool developed from the NHMRC Guideline could assist greatly in overcoming communication and knowledge obstacles described in this thesis; and a suggestion of what this ready-reference tool could look like is included.

Passively disseminating clinical practice guidelines has been ineffective at optimising thromboprophylaxis use to date, both in Tasmania and interstate, potentially leaving many patients exposed to a higher risk of VTE. A clear understanding of the factors that influence thromboprophylaxis prescribing and guideline adoption is essential. Together, the studies 
contained in this thesis identified many barriers to thromboprophylaxis use and guideline adoption, including: concerns over bleeding complications (and associated potential underreporting), the scope of industry influence, and the threat of guidelines being too prescriptive, threatening professional autonomy and thereby posing a potential medico-legal liability to surgeons. These barriers should be addressed in forthcoming editions of the NHMRC Guideline to encourage uptake of its recommendations and ensure optimal outcomes for patients. 


\section{Publications}

Mirkazemi C, Bereznicki LRE, Peterson GM. Thromboprophylaxis following hip and knee arthroplasty. Internal Medicine Journal. 2013; 43(2):124-129

Mirkazemi C, Bereznicki LR, Peterson GM. Are the national orthopaedic thromboprophylaxis guidelines appropriate? ANZ Journal of Surgery. 2012;82(12):913-917.

Mirkazemi C, Bereznicki LRE, Peterson GM. Response to Re: Are the national orthopaedic thromboprophylaxis guidelines appropriate? ANZ Journal of Surgery. 2013;83(5):395-396. 


\section{LIST OF FigURES}

Figure 1. Proportion of suspected DVT confirmed with venography.......................................... 4

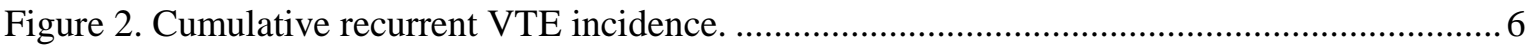

Figure 3. Pharmacological prophylaxis and VTE incidence following major orthopaedic surgery. 11

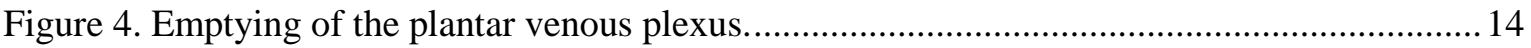

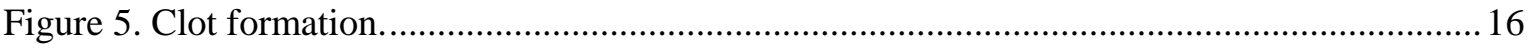

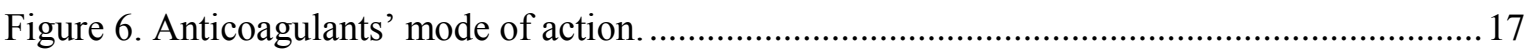

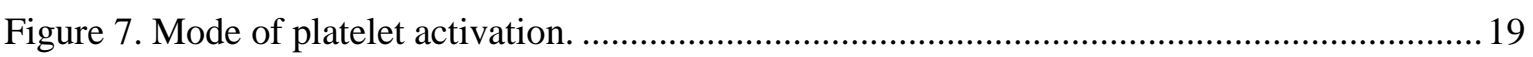

Figure 8. Graphic representation of patients screened and proportioned to study groups. .............. 20

Figure 9. Graphic representation of patients screened and proportioned to study groups. ...............21

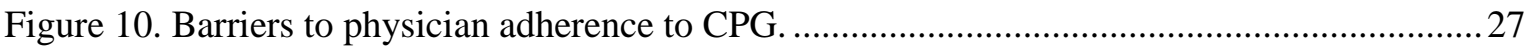

Figure 11. Factors that increase the likelihood of prescribing pharmacological prophylaxis ..........28

Figure 12. Factors that increase the likelihood of prescribing pharmacological agents (1999),......29

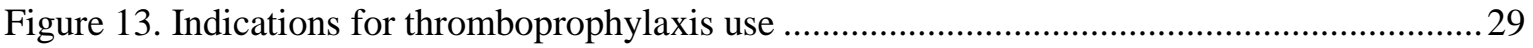

Figure 14. Factors that might decrease the use of pharmacological prophylaxis.............................. 30

Figure 15. Barriers to pharmacological thromboprophylaxis use .................................................. 31

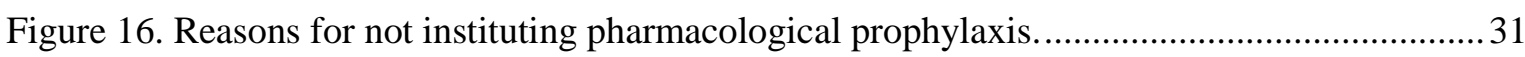

Figure 17. Proportion discharged home with ACCP recommended prophylaxis ............................. 38

Figure 18. Proportion of patients who received NHMRC recommended prophylaxis ......................46

Figure 19. Proportion of patients who received NHMRC recommended prophylaxis ..................... 47

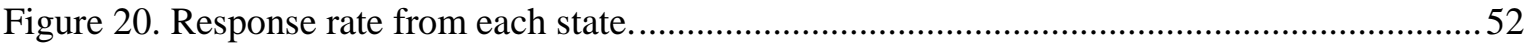

Figure 21. Basis of thromboprophylaxis protocols in responding hospitals .....................................54

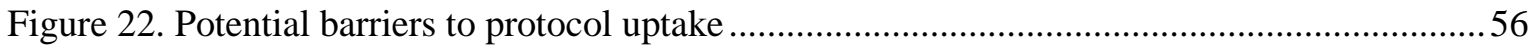

Figure 23. Basis of thromboprophylaxis protocols in responding hospitals ....................................56

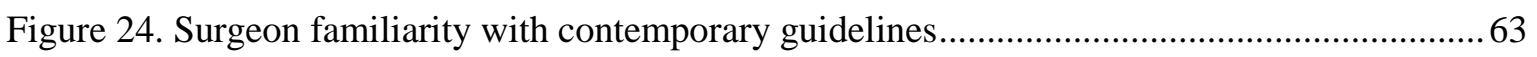

Figure 25. Comparison of RHH prescribing practices with the ACCP $9^{\text {th }}$ Ed. Guideline................. 95

Figure 26. Comparison of multicentre prescribing practices with the ACCP $9^{\text {th }}$ Ed. Guideline...... 96

Figure 27. Comparison of multicentre prescribing practices with the ACCP $9^{\text {th }}$ Ed. Guideline...... 96

Figure 28. Surgeon familiarity with contemporary guidelines..................................................... 105

Figure 29. Proportion of surgeons very familiar with contemporary guidelines. .......................... 118 


\section{LIST OF TABLES}

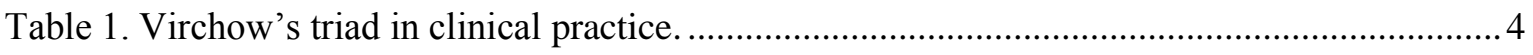

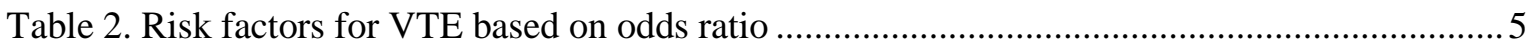

Table 3. Relative cumulative risk of recurrent VTE within four years ............................................. 6

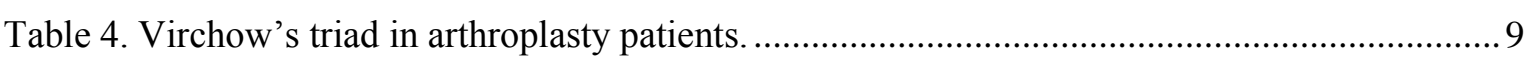

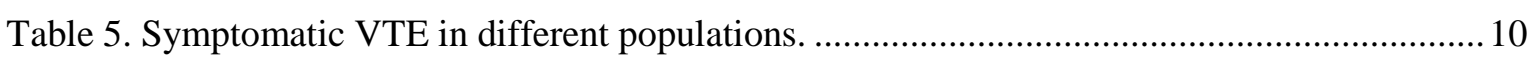

Table 6. Australian incidence of symptomatic VTE following arthroplasty.................................... 11

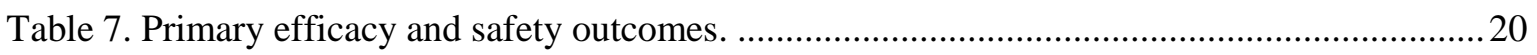

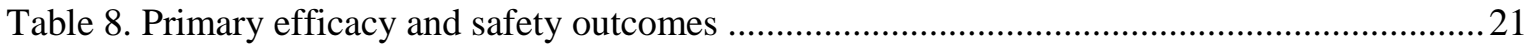

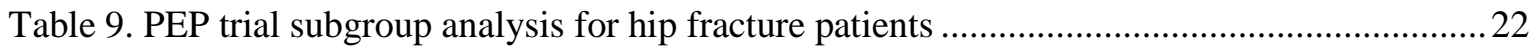

Table 10. Thromboprophylaxis recommendations in late 2009.................................................... 24

Table 11. Updated AAOS, ACCP and ASA thromboprophylaxis recommendations.......................25

Table 12. Guideline recommendations during the study period. ….................................................. 34

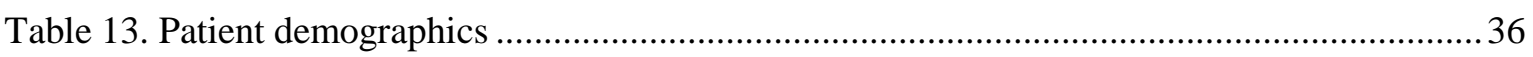

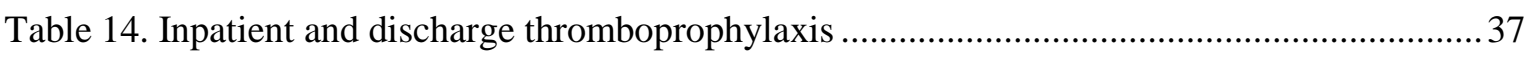

Table 15. Symptomatic postoperative VTE incidence .................................................................... 39

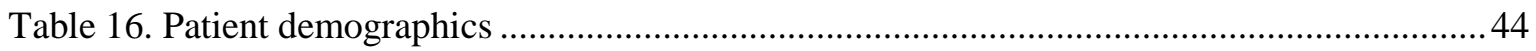

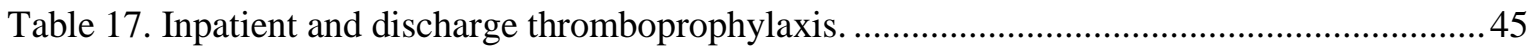

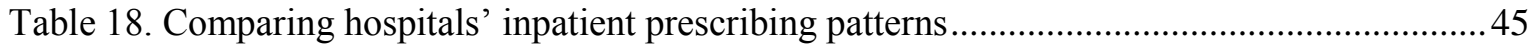

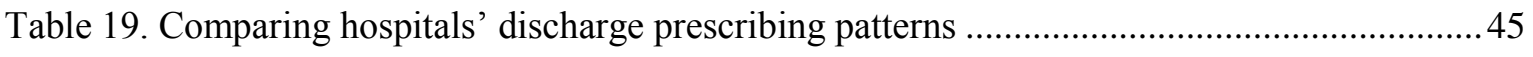

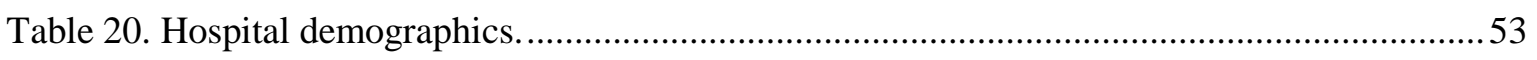

Table 21. Reported hospital policies to encourage protocol adherence ............................................55

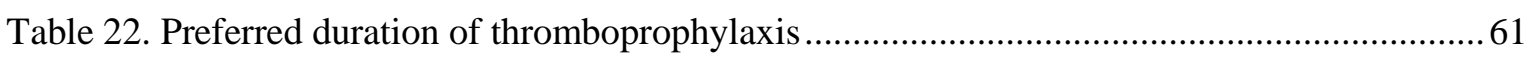

Table 23. Patient factors that prompt pharmacological prophylaxis use........................................... 62

Table 24. Perceptions that limit pharmacological prophylaxis use ..................................................62

Table 25. Why the NHMRC recommendations were deemed inappropriate...................................... 64

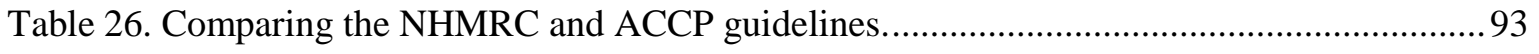

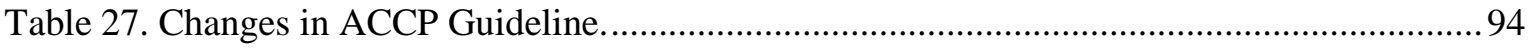

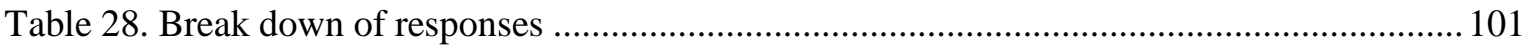

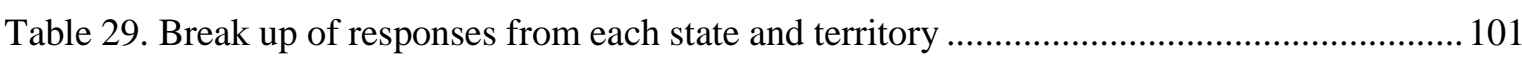

Table 30. Primary pharmacological preferences (\%) between ASA and non-ASA members ........ 102

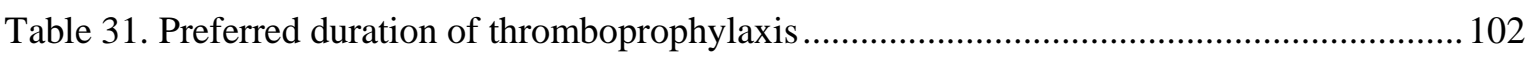

Table 32. Opinions on efficacy of pharmacological prophylaxis................................................... 103 
Table 33. Patient factors that prompt surgeons to prescribe pharmacological prophylaxis ............ 104

Table 34. Factors that limit pharmacological prophylaxis use........................................................ 104

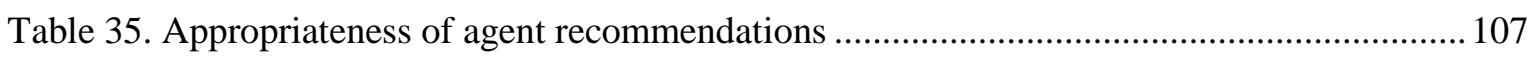

Table 36. ASA members' opinions on efficacy of pharmacological prophylaxis. …...................... 116

Table 37. Patient factors that prompt surgeons to prescribe pharmacological prophylaxis ............ 117

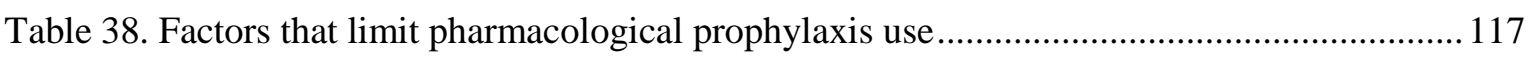




\section{AbBreviations}

$\begin{array}{ll}\sim & \text { approximately } \\ \% & \text { per cent }\end{array}$

AAOS American Academy of Orthopaedic Surgeons

ACCP American College of Chest Physicians

ADP adenosine diphosphate

ANZWP Australia and New Zealand Working Party

AOA Australian Orthopaedic Association

ASA Arthroplasty Society of Australia

ATP adenosine triphosphate

AUD Australian dollar

BMI body mass index

cAMP cyclic adenosine monophosphate

cf. compared with/to

CI confidence interval

CNM clinical nurse manager (orthopaedic)

CPG clinical practice guideline

$\mathrm{CrCl}$ creatinine clearance

DC discharge

DHHS Department of Health and Human Services

DVT deep vein thrombosis

e.g. for example

GCS graduated compression stockings

GIT gastrointestinal tract

GP general practitioner

HA hip arthroplasty

HRT hormone replacement therapy

IPCD intermittent pneumatic compression device

HOD head of department

JD junior orthopaedic doctor

KA knee arthroplasty

LMWH low molecular weight heparin

LOS length of stay

min. minimum

NOA novel oral agents

NHMRC National Health and Medical Research Council 


$\begin{array}{ll}\text { NICE } & \text { National Institute of Clinical Excellence } \\ \text { NSAID } & \text { non-steroidal anti-inflammatory drug } \\ \text { NSW } & \text { New South Wales } \\ \text { NZ } & \text { New Zealand } \\ \text { PCI } & \text { percutaneous coronary intervention } \\ \text { PE } & \text { pulmonary embolism } \\ \text { PGI }_{2} & \text { prostacyclin } \\ \text { PTS } & \text { post thrombotic syndrome } \\ \text { RCT } & \text { randomised controlled trial } \\ \text { RHH } & \text { Royal Hobart Hospital } \\ \text { SD } & \text { standard deviation } \\ \text { SeCr } & \text { serum creatinine } \\ \text { TEDs } & \text { thromboembolic deterrent stockings } \\ \text { TXA } & \text { thromboxane } A_{2} \\ \text { UFH } & \text { unfractionated heparin } \\ \text { VFP } & \text { venous foot pump } \\ \text { VKA } & \text { vitamin K antagonist } \\ \text { vs. } & \text { versus } \\ \text { VTE } & \text { venous thromboembolism } \\ \text { yr } & \text { year }\end{array}$




\section{CHAPTER ONE: INTRODUCTION}

\subsection{Thesis Background}

Venous thromboemboli (VTE) are the most common cause of preventable death in Australian hospitals. ${ }^{1}$ There are a number of factors that increase a person's risk of developing VTE, including advanced age, obesity, medications and surgery. ${ }^{2}$ Two such surgical procedures that significantly increase VTE risk, independent of other risk factors, are hip and knee arthroplasty. ${ }^{2}$

Fortunately, there are mechanical and pharmacological measures that can be employed to prevent VTE, known collectively as thromboprophylaxis. The American Agency for Healthcare Research and Quality refers to these measures as their 'number one patient safety practice'. ${ }^{3}$ Many national and international bodies have released thromboprophylaxis guidelines in recent years; however, despite their wide promulgation, studies indicate that thromboprophylaxis is underutilised both in Australia and internationally. ${ }^{4-11}$

This thesis reports on work that investigated thromboprophylaxis prescribing following hip and knee arthroplasty in Australia, as well as barriers and enablers to its widespread use.

\subsection{Research Methodology}

A mixed methods approach to the overall research methodology was utilised, gathering together both quantitative and qualitative data to provide new insights into what drives thromboprophylaxis prescribing following hip and knee arthroplasty. The quantitative data included retrospective reviews and surveys. The qualitative data included open ended survey data and semi-structured interviews.

A mixed methods approach was utilised to overcome the potential deficiencies inherent in both qualitative and quantitative research methods. For example, quantitative research methods can sometimes produce findings of 'little consequence' because they tend to generalise findings rather than taking into account peoples' varied experiences or the way they interpret and make sense of their experiences. ${ }^{12}$ On the other hand, the often relatively small sample sizes used in qualitative research methods can sometimes limit the generalisability of their findings. Regardless of the type of data utilised in a study, exclusive reliance on only one dataset to answer a research question can bias or distort one's picture of the particular slice of reality being investigated. ${ }^{13}$

Triangulation attempts to map out, or explain more fully, the richness and complexity of human behaviour by studying it from more than one standpoint. ${ }^{13}$ Methodological triangulation (the use of two or more different kinds of methods in a single line of inquiry) was employed in this 
research to provide a more complete understanding of the slice of reality being studied. ${ }^{14}$ For example, surgeons' perceptions of thromboprophylaxis were captured in closed and free-text survey questions, while semi-structured interviews with surgeons (who may or may not have participated in the survey) and other stakeholders (e.g. general practitioners) also contributed information on how thromboprophylaxis following hip and knee arthroplasty is perceived and prescribed. In this way a more holistic view of prescribing and factors that influence prescribing was obtained.

\subsection{Significance of the Research}

Passively disseminating clinical practice guidelines has been ineffective at improving thromboprophylaxis use in Australia. ${ }^{15}$ A clear understanding of the factors that influence thromboprophylaxis prescribing and guideline adoption is essential for the optimal use of thromboprophylaxis, and subsequent optimal care and outcomes for patients undergoing hip and knee arthroplasty in Australia. Significantly, this research explored these factors in greater depth than previously reported. The findings and benefits of this research will accrue indirectly and in the long term.

\subsection{Limitations of the Research}

This research explored thromboprophylaxis prescribing patterns and factors that influence them. As such the research was not designed to examine, at least as a primary outcome, the effectiveness of thromboprophylaxis in Australian arthroplasty patients.

\subsection{Ethics}

The research associated with this thesis abides by the international and Australian codes on human and animal experimentation. This research received approval from the Human Research Ethics Committee (Tasmania) Network (approval reference numbers: H0010881, H0011165 and H0011265, including amendments), the Hunter New England Research Ethics \& Governance Unit (11/12/14/5.07), the Austin Health Research Ethics Committee (EER 04471) and the Western Health Ethics Committee (QA 2012.34). 


\section{CHAPTER TWO: THROMBOPROPHYLAXIS FOLLOWING HIP AND KNEE ARTHROPLASTY}

\subsection{Thromboemboli}

A blood clot can be classified as either an arterial or venous thromboembolism depending on the type of blood vessel it originates from. While both are composed of platelets and fibrin, arterial thrombi tend to occur at sites of arterial plaque rupture where blood flow is high. ${ }^{16}$ They are usually platelet-rich and are referred to as 'white thrombi'. In contrast, venous thromboemboli originate in the venous system where blood flow and shear rates are low. Furthermore, they tend to occur at sites where the vein wall is often healthy. Venous thromboemboli (VTE) are rich in red blood cells and fibrin, and are often termed 'red thrombi'. ${ }^{16}$ This thesis specifically focuses on work exploring VTE prevention following hip and knee arthroplasty.

\subsubsection{Venous Thromboembolism}

VTE is an evolving, multi-factorial disease that can result in either partial or complete block of blood flow to the surrounding tissues. ${ }^{17}$ Deep vein thrombosis (DVT) and pulmonary embolism (PE) are two aspects of this one disease process. A DVT usually starts in the calf veins, from where it may extend to the proximal veins; it can then subsequently break free to travel to the pulmonary blood vessels to result in a PE. ${ }^{17,18}$ In general, PE occur less commonly, with approximately one third of symptomatic VTE manifesting as PE and two thirds manifesting as DVT alone. ${ }^{19,20}$

Many VTE will occur without symptoms and are known as asymptomatic clots. VTE which do become symptomatic will vary in their presentation depending on both the type and severity of the clot. Symptoms of DVT include pain, tenderness, erythema, and swelling. Furthermore, DVTs can lead to chronic venous insufficiency and eventually venous ulcers. Symptoms of PE can include shortness of breath, bloody sputum, chest pain, faintness, and heart failure. Massive PE are life threatening and remain one of the most common causes of preventable death in Australia. ${ }^{17}$

The manifestation of symptoms depends on a number of factors. These include: the extent of thrombosis, the adequacy of collateral blood vessels, and the severity of the associated vascular occlusion and inflammation. ${ }^{18}$ Additionally, the physiological capacity of the patient to tolerate the thrombosis plays an important role in not only the manifestation of symptoms, but also the clinical outcome. For example, a moderately sized PE may cause no symptoms in an otherwise healthy individual, but severe symptoms or even death may result from the same PE in an individual with severe cardiopulmonary disease. ${ }^{18}$ 


\subsubsection{Pathophysiology}

Thrombus formation and propagation depend on the presence of three interdependent factors. These factors are collectively named Virchow's triad after the nineteenth century German pathologist Rudolph Virchow who is credited with 'discovering' PE. ${ }^{21}$ Virchow's triad of factors are endothelial injury or dysfunction, haemostasis, and hypercoagulability. Elements that increase VTE risk through instigating one or more of Virchow's triad are collectively referred to as VTE risk factors (Table 1).

Table 1. Virchow's triad in clinical practice. ${ }^{22}$

Factor $\quad$ Clinical examples

Hypercoagulability

Oestrogen therapy, thrombophilia, previous VTE, pregnancy, cancer

Haemostasis

Cancer, obesity, major surgery, hip or leg fracture, myocardial infarction

Endothelial injury or dysfunction

Trauma, major surgery, hip or leg fracture

The combination of one or more of Virchow's triad in a person greatly increases their VTE risk. $^{22}$ Consequently, a patient with a suspected DVT is more likely to have their diagnosis confirmed by objective testing if they have one or more of these risk factors (Figure 1).

Figure 1. Proportion of suspected DVT confirmed with venography.

Source: Anderson et al (2003). ${ }^{2}$

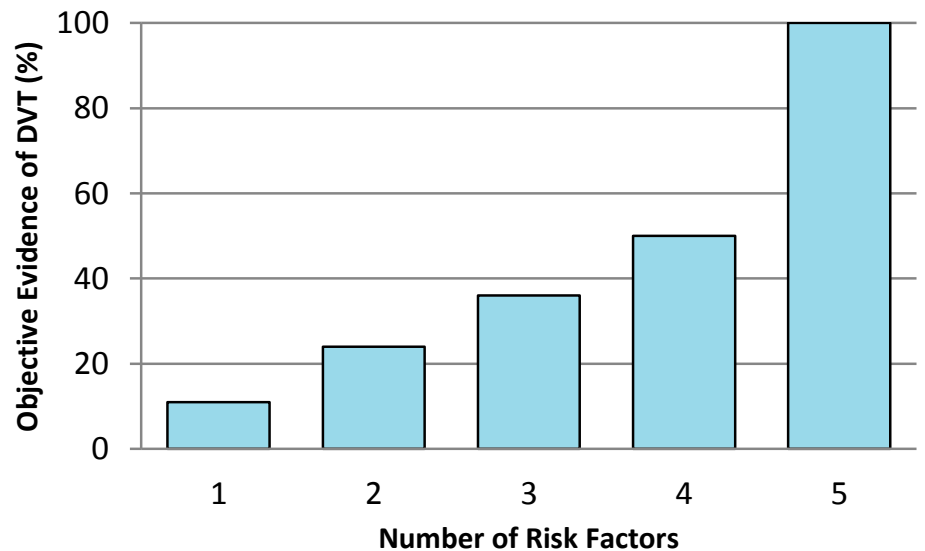

Although risk factors increase the risk of developing VTE, not all factors have the same predictive weight. $^{2}$ One of the strongest risk factors for VTE development is surgery, and in particular hip and knee arthroplasty (Table 2). 
Table 2. Risk factors for VTE based on odds ratio. ${ }^{2}$

\section{Strong Risk Factors (OR>10)}

Hip or knee fracture

Hip or knee arthroplasty

Major general surgery

Major trauma

Spinal cord injury

Depending on the type of risk factors present in a person who develops VTE, their clot can be classified as either provoked or unprovoked. Provoked VTE are those that occur secondary to transient risk factors (e.g. trauma and pregnancy), whereas unprovoked VTE are defined as being secondary to persistent risk factors (e.g. thrombophilia). ${ }^{23}$ Surgery is a transient risk factor, and therefore a symptomatic VTE following orthopaedic surgery is usually classified as a provoked VTE.

\subsubsection{Clinical Sequelae and Consequences}

The main cost of VTE is the loss of healthy life that it entails, both acutely and chronically. Acutely, people with postoperative VTE are likely to remain out of work longer than anticipated. ${ }^{24-}$ 27 This can be due to a combination of the hospital stay (or being promptly readmitted after presentation to outpatient clinics/emergency departments) and the substantial physical limitations which they often experience during the rehabilitation and recovery period. Additionally, patients who develop postoperative VTE usually require anticoagulation for extended periods of time, thereby exposing them to its inherent risks, and treatment costs. Combined, these factors can easily result in a patient having to endure a substantial VTE associated cost burden postoperatively. ${ }^{27}$

The long term morbidity associated with VTE can be as equally burdensome as the acute phase symptoms. Long term complications most commonly include recurrent VTE and post thrombotic syndrome (which can lead to ulcers and cellulitis). ${ }^{27,28}$ Most complications occur weeks to months after the initial clot, although ulceration can occur months to years later.

In addition to the acute and chronic repercussions described above, VTE has the potential to be lethal. It is estimated that $1 \%$ of all patients admitted to hospital will die from it, and it accounts for $10 \%$ of all hospital deaths. ${ }^{1,17}$ 


\subsubsection{Recurrent Venous Thromboemboli}

A previous history of VTE significantly increases a person's risk of developing subsequent VTE. ${ }^{29}$ It is estimated that a person is approximately 40 times more likely to suffer VTE within six months of their initial episode compared with a previously unaffected person (3.6 to $8.0 \%$ vs. $0.08 \%$, respectively). ${ }^{18,30-32}$

Observational data indicate that this risk is not identical for everyone and that the risk of recurrent VTE in an individual patient can be estimated from a variety of interacting clinical, laboratory and imaging findings. ${ }^{33}$ Although there are conflicting views as to how accurately these findings can predict one's risk, one factor which is unanimously agreed to affect VTE recurrence is the nature of the initial VTE (provoked or unprovoked). ${ }^{31,33-35}$ Significantly, surgically provoked VTE is associated with approximately half the risk of recurrence compared to unprovoked VTE (Table 3). ${ }^{35}$

Table 3. Relative cumulative risk of recurrent VTE within four years ..$^{35}$
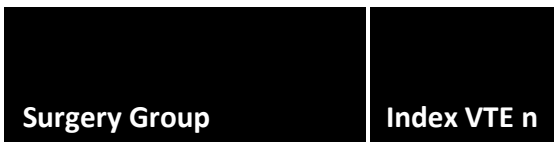

\section{Recurrent VTE \% after} Provoked Index VTE Unprovoked Index VTE $(95 \% \mathrm{Cl})$ $(95 \% \mathrm{Cl})$

Difference in relative risk

Multiple surgical procedures combined

11,797

$7.6(7.0-8.2)$

$5.9(5.0-7.1)$

$7.8(6.6-9.3)$
$14.7(14.2-15.1)$

14.3 (13.5-15.3)
$14.4(13.5-15.3)$

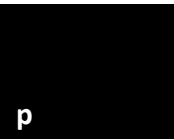

\begin{tabular}{l|l}
$48 \%$ & 0.001
\end{tabular}

\begin{tabular}{l|l}
$59 \%$ & 0.001
\end{tabular}

\begin{tabular}{l|l}
$45 \%$ & 0.001
\end{tabular}

The risk of recurrent VTE continues for years after the initial episode irrespective of whether the initial VTE was provoked or unprovoked; however, the cumulative risk is lower for provoked VTE. In both settings, the risk of recurrence is highest in the first few days to weeks after an initial event, with a more gradual increase in the cumulative recurrence rate thereafter (Figure 2). ${ }^{35}$

Figure 2. Cumulative recurrent VTE incidence.

Kaplan-Meier plot of the incidence of recurrent VTE in a knee arthroplasty provoked VTE group versus a matched unprovoked VTE group.

Source: White et al (2010). ${ }^{35}$

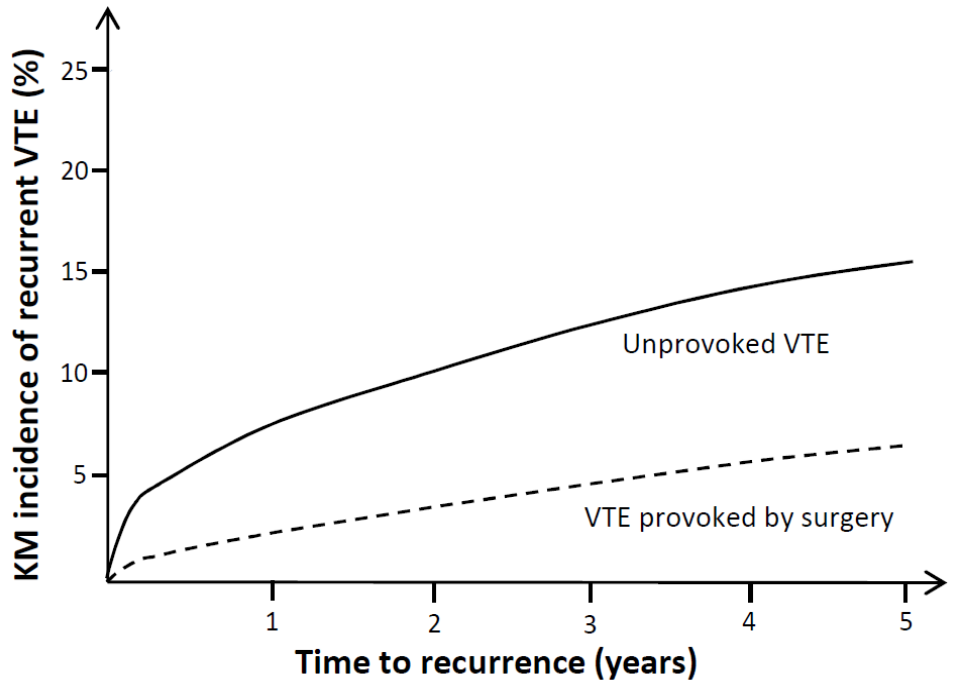


It is generally accepted that the type of VTE (PE or DVT) strongly predicts the type of VTE manifested on recurrence. ${ }^{18,32,36,37}$ That is, recurrent VTE after a DVT is most likely to be in the form of another DVT, and the is same true of PE. Some recent data suggests there may be no significant bias; however, this requires further investigation. ${ }^{38}$

\subsubsection{Post Thrombotic Syndrome}

Post thrombotic syndrome (PTS) is the most common long term complication of DVT. ${ }^{39}$ This chronic syndrome can vary significantly between patients; however, classic symptoms include pain, swelling, skin depigmentation, and itchiness. ${ }^{39}$ It has been hypothesised that PTS occurs as a result of valve damage and outflow obstruction following a DVT; this can lead to vein dilatation, fluid accumulation, venous hypertension, abnormal microcirculation, and finally tissue hypoxia. ${ }^{40}$

People who experience PTS can incur significant morbidity (including decreased mobility) and may potentially require chronic medical and home nursing care. Accordingly, patients who experience PTS report decreased disease-specific quality of life scores which are, on average, similar or worse than those reported by patients with osteoarthritis or chronic lung disease. ${ }^{41}$ These scores worsen with increasing PTS severity. ${ }^{40}$

PTS has been reported to occur with varying frequency in patients following VTE, both symptomatic and asymptomatic, provoked and unprovoked. ${ }^{42}$ It is estimated that $23 \%$ to $60 \%$ of people will develop PTS and up to $10 \%$ will develop venous ulcers within 2 years of an unprovoked VTE, with cumulative rates further increasing thereafter. ${ }^{43}$ PTS incidence following provoked VTE is less frequent; only an estimated 5\% to $10 \%$ of arthroplasty patients report severe PTS symptoms within two to four years of surgery. ${ }^{44}$

One possible explanation why PTS is less common after a surgically provoked VTE than an unprovoked VTE is that a person who develops an unprovoked VTE is generally more likely to have multiple comorbidities compared to a patient healthy enough to undergo surgery. This means their physiological capacity to tolerate a thrombosis will be reduced, and thus their likelihood of developing PTS will be increased. Additionally, the use of thromboprophylaxis postoperatively not only reduces the incidence of VTE, but may also reduce the size of a thrombus in those who do develop one; and small thrombi are less likely to cause the venous obstruction and valvular damage associated with PTS. ${ }^{45}$

Treatment options for PTS are limited to nocturnal leg elevation, vascular surgery, pain management and weight loss; consequently, VTE prevention is crucial to reducing the PTS burden on individuals and society.

\subsubsection{Mortality}

Although DVT itself is unlikely to be lethal, if it is left untreated it can potentially dislodge and move into the lungs to cause PE, which can be fatal. Accurately determining how often people die 
as a result of VTE is difficult, particularly because many patients do not die immediately. Instead, patients will often have nonspecific symptoms and for this reason the diagnosis of VTE associated death is often delayed or missed entirely without an autopsy. ${ }^{18}$

Postoperative VTE mortality data is even more limited than general VTE mortality data and many of the studies reporting post-arthroplasty complications are limited by their retrospective nature. Those studies choosing to only investigate in-hospital fatality fail to capture deaths attributable to VTE a few days or weeks out from discharge. Conversely, choosing excessively long study periods can artificially inflate mortality rates by including deaths due to VTE that are independent of the surgery itself.

Nevertheless, it is estimated that the general mortality risk within one month of DVT is $6 \%$ and $12 \%$ following PE. ${ }^{19,46}$ Notably, a patient's risk factors and the nature of the clot they develop (provoked or unprovoked) both influence their risk of dying following VTE. ${ }^{47,48}$ For example, provoked VTE are less likely to result in death than unprovoked VTE. The 90-day mortality risk following hip and knee arthroplasty is reported to be $0.2 \%$ to $0.3 \%$, only one third of which is attributed to VTE. ${ }^{49,50}$

Furthermore, observational data indicate that surgery factors can also impact on mortality risk following hip and knee arthroplasty. For example, the risk of dying is significantly higher following hip arthroplasty compared to knee arthroplasty $(3.0 \%$ vs. $0.1 \%) .{ }^{47,51}$ Moreover, Australian data indicate first-year cumulative mortality to be higher following total conventional hip arthroplasty compared to total resurfacing hip arthroplasty ${ }^{*}(1.2 \%$ vs. $0.2 \%)$, and higher following total knee arthroplasty compared to partial knee arthroplasty (1.0\% vs. $0.4 \%) .{ }^{52}$ The highest risk period for postoperative fatal PE appears to be 3 to 7 days after surgery. ${ }^{18}$

\footnotetext{
* Unlike traditional hip replacement, hip resurfacing does not replace the ball of the femur with a metal or ceramic ball. Instead, the damaged hip ball is reshaped and capped with a metal prosthesis. The damaged hip socket is also fitted with a metal prosthesis, similar to what is used in a conventional hip replacement.
} 


\subsection{Hip and Knee Arthroplasty}

Hip and knee arthroplasty (HA and KA respectively) are increasingly common procedures in Australia. During these surgeries an arthritic or damaged joint is removed and replaced with an artificial joint or prosthesis. In 2011 approximately 83,000 arthroplasties were conducted in Australia, with $28.4 \%$ of patients requiring more than one procedure. ${ }^{53,54}$ There is a general trend towards more knee arthroplasties being conducted annually than hip arthroplasties, and although the data is limited (it relies on self-reporting by surgeons and hospitals), there is an upward trend in both procedures each year. ${ }^{53}$

Hip and knee arthroplasty are particularly strong risk factors for VTE because all three aspects of Virchow's triad occur during the pre-, peri- and postoperative period (Table 4).

Table 4. Virchow's triad in arthroplasty patients. ${ }^{22,55-57}$

\begin{tabular}{|l|l|l|}
\hline Operative stage & Example & Virchow factor \\
\hline Preoperatively & Limb immobility caused by fractures or breaks. & $\begin{array}{l}\text { Haemostasis } \\
\text { Hypercoagulability }\end{array}$ \\
\hline $\begin{array}{l}\text { Pre- and peri- } \\
\text { operatively }\end{array}$ & $\begin{array}{l}\text { Knee arthroplasties performed with a thigh tourniquet and the } \\
\text { knee in a flexed and sub-luxated position can lead to venous } \\
\text { stasis. }\end{array}$ & Haemostasis \\
\hline Perioperatively & $\begin{array}{l}\text { Endothelium can be injured during positioning and } \\
\text { manipulation of the extremity. }\end{array}$ & Endothelial injury/dysfunction \\
\hline $\begin{array}{l}\text { Blood vessel wall distension caused by blood pooling, } \\
\text { particularly with tourniquet use, can cause endothelial damage } \\
\text { and lead to clotting activation. }\end{array}$ & $\begin{array}{l}\text { Hypercoagulability } \\
\text { Endothelial injury/dysfunction }\end{array}$ \\
\hline $\begin{array}{l}\text { Bone cement may cause thermal injury. } \\
\text { Damaged bone marrow activates clotting factors released } \\
\text { during the operation. These factors often aggregate in regions } \\
\text { of venous stasis. }\end{array}$ & Hypercoagulability \\
\hline $\begin{array}{l}\text { Blood loss reduces endogenous anticoagulants and inhibits the } \\
\text { fibrinolytic system. }\end{array}$ & Hypercoagulability \\
\hline $\begin{array}{l}\text { Localised postoperative swelling and immobility can lead to } \\
\text { venous stasis. }\end{array}$ & Haemostasis \\
\hline Postoperatively & & \\
\hline
\end{tabular}

\subsubsection{Postoperative Venous Thromboemboli}

Although it is difficult to determine the baseline incidence of symptomatic VTE following hip and knee arthroplasty, it is generally agreed that arthroplasty patients are at an increased risk of developing VTE compared to the population at large (Table 5). A recent study identified a 13-fold and 14-fold increased VTE risk in hip and knee patients (respectively) compared with a matched cohort. $^{58}$ 
Table 5. Symptomatic VTE in different populations.

Population

General population

Knee and hip arthroplasty patients
Symptomatic VTE incidence (per 100,000)

$83-114$

$1,200-5,300$
References

$6-8,50,60,61$

Studies report that up to $40 \%$ of arthroplasty patients experience VTE within a few days of surgery; however, most are asymptomatic. ${ }^{22,63}$ The clinical relevance of asymptomatic VTE is unknown because their association with symptomatic VTE is uncertain. The most reliable approach to determine the relationship between the symptomatic and asymptomatic VTE is to blind a cohort of patients and health care providers to the results of venography, and compare short and long-term outcomes in these patients. Some clinicians argue however, that it is unethical to conceal these results because there is a possibility of asymptomatic VTE extending or propagating around the body. ${ }^{64,65}$

An alternative approach would be to examine the relationship between asymptomatic VTE and the incidence of VTE associated clinical sequelae. Although this approach can be biased by the use of anticoagulants to treat asymptomatic VTE, it has been studied in a variety of settings. There is evidence that asymptomatic DVT is associated with the development of PTS in medical patients, however not so in patients who undergo lower limb surgery. ${ }^{42,45,66,67}$ This may be due to an inherent difference in the nature of provoked and unprovoked VTE, and the physiological capacity of the afflicted patients.

While the clinical relevance of symptomatic VTE is generally accepted, it is difficult to ascertain its true baseline incidence. This is because there have been no large placebo controlled trials or well-designed cohort studies conducted in recent years. Furthermore, those placebo controlled trials that were conducted in previous years are not relevant to current practice as pre-, peri- and postoperative surgical care following hip and knee arthroplasty has changed considerably over the years. ${ }^{68}$ For example, the average length of stay in hospital following surgery has decreased markedly from almost 17 days in 1992 to 9 days in 1999, and more recently from 3.2 days in 2008 to 2 days in 2009 . $^{69-72}$

Additionally, the patients themselves have also changed; younger, healthier patients are having surgery, with a greater emphasis being placed on ensuring good postoperative function and mobility. ${ }^{73,74}$ Furthermore, from 2003 onwards there has been a shift in surgical techniques to less invasive (and therefore potentially less thrombogenic) techniques. ${ }^{68,70}$

Although it is not possible to directly determine a baseline symptomatic VTE risk for arthroplasty patients, it is possible to estimate it. A review of trials conducted after 2003 reported symptomatic VTE risk in patients using pharmacological thromboprophylaxis to be approximately $1.2 \%$ within the first 7 to 14 days following surgery (0.8\% DVT, $0.4 \% \mathrm{PE}){ }^{68}$ Several reviews have estimated that pharmacological prophylaxis reduces relative VTE incidence (symptomatic and 
asymptomatic) by $50 \%$ to $70 \%$, and potentially up to $85 \% .{ }^{75-78}$ Extrapolating these two findings, we can estimate that the contemporary baseline VTE risk following arthroplasty is $2.9 \%$ within the first 7 to 14 days following surgery.

Data suggests that the increased risk of VTE continues for up to three months after surgery both with and without thromboprophylaxis. ${ }^{62,68,79}$ Therefore, the true cumulative baseline risk for symptomatic VTE following arthroplasty is likely to be higher than $2.9 \%$ (Figure 3). The reported three-month symptomatic VTE incidence rate in Australia is $3.8 \%$ to $5.0 \%$ following arthroplasty, with most occurring within the first month following surgery (Table 5). ${ }^{51}$

Figure 3. Pharmacological prophylaxis and VTE incidence following major orthopaedic surgery.

Observational data is represented for the cumulative incidence rate resulting from high use of pharmacological prophylaxis in the first 7 to 14 days postoperatively.

NB: The pharmacological prophylaxis in this instance is low molecular weight heparin (LMWH).

Source: Falck-Ytter et al (2012). ${ }^{68}$

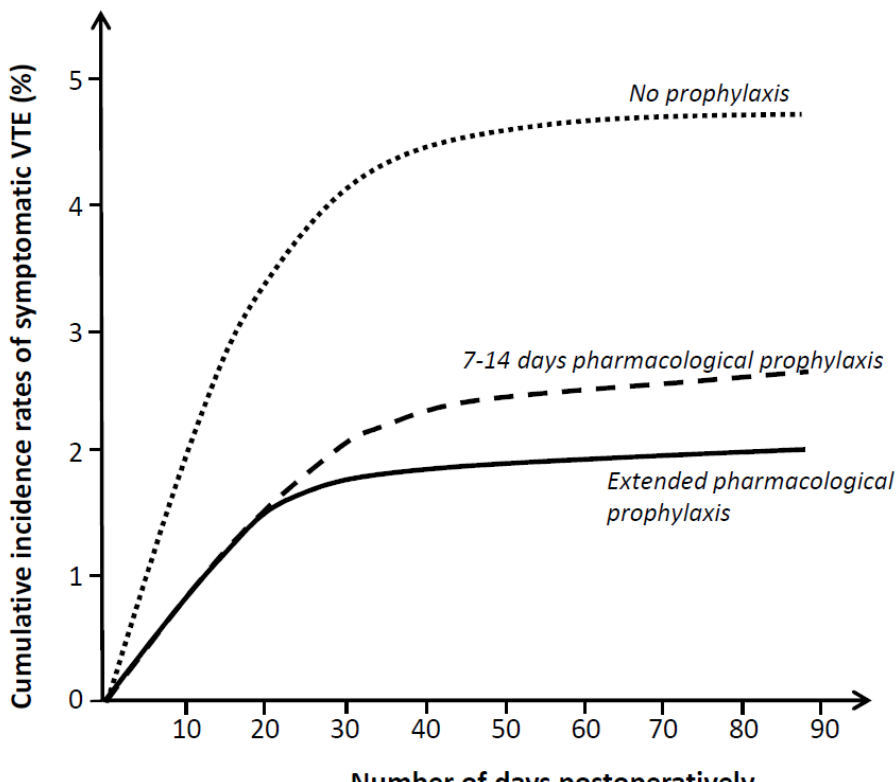

Number of days postoperatively

Table 6. Australian incidence of symptomatic VTE following arthroplasty.

\begin{tabular}{|c|c|c|c|c|}
\hline Source & $\mathbf{n}$ & Data period & Thromboprophylaxis & Incidence \\
\hline $\begin{array}{l}\text { O'Reilley et } \\
\text { al } 2005^{63}\end{array}$ & $\begin{array}{l}5999 \\
\mathrm{HA} / \mathrm{KA}\end{array}$ & $1995-2001$ & $\begin{array}{l}\text { Seven days LMWH or VKA + GCS. } \\
\text { Prophylaxis then changed based on VTE investigations. }\end{array}$ & $\begin{array}{l}1.9 \% \text { non-fatal } \\
\text { inpatient PE }\end{array}$ \\
\hline $\begin{array}{l}\text { Hitos et al } \\
2006^{6}\end{array}$ & $\begin{array}{l}283 \\
K A\end{array}$ & 1996-2004 & $\begin{array}{l}\text { All patients received mechanical } \pm \text { pharmacological } \\
\text { prophylaxis. } \\
82.5-100 \% \text { given anticoagulant + mechanical prophylaxis. }\end{array}$ & $\begin{array}{l}3 \text { month VTE } \\
\text { incidence: } 3.9 \%\end{array}$ \\
\hline $\begin{array}{l}\text { Hitos et al } \\
2009^{7}\end{array}$ & $\begin{array}{l}321 \\
H A\end{array}$ & 1996-2004 & $\begin{array}{l}74-100 \% \text { received mechanical + pharmacological } \\
\text { prophylaxis. }\end{array}$ & $\begin{array}{l}3 \text { month VTE } \\
\text { incidence: } 3.8 \%\end{array}$ \\
\hline $\begin{array}{l}\text { Eikelboom } \\
\text { et al } 2004^{8}\end{array}$ & $\begin{array}{l}157 \\
\mathrm{HA} / \mathrm{KA}\end{array}$ & 2002 & $\begin{array}{l}51.5 \% \text { received } \mathrm{LMWH} \text { or VKA postoperatively, most of the } \\
\text { rest received mechanical prophylaxis or aspirin. } \\
56.6 \% \text { received prophylaxis post-discharge. }\end{array}$ & $\begin{array}{l}3 \text { month VTE } \\
\text { incidence: } 5.3 \%\end{array}$ \\
\hline $\begin{array}{l}\text { Buchan et } \\
\text { al } 2008^{80}\end{array}$ & $\begin{array}{l}246 \\
\mathrm{HA} / \mathrm{KA}\end{array}$ & 2004-2005 & $\begin{array}{l}98 \% \text { received anticoagulants in hospital. } \\
8.2 \% \text { receiving an anticoagulant post-discharge. }\end{array}$ & $\begin{array}{l}3 \text { month PE } \\
\text { incidence: } 2.8 \% \\
\text { non-fatal, } 0.4 \% \\
\text { fatal }\end{array}$ \\
\hline
\end{tabular}

NB: $L M W H=$ low molecular weight heparin (e.g. enoxaparin), VKA = vitamin $\mathrm{K}$ antagonist (e.g. warfarin), $\mathrm{GCS}=$ graduated compression stockings, $\mathrm{HA}=$ hip arthroplasty, $\mathrm{KA}=$ knee arthroplasty. 
There can be significant variation in the VTE incidence reported in studies. ${ }^{81,82}$ Such variations in VTE may be in part due to thromboprophylaxis differences; however there are many other potential influencing factors, some of which are described briefly below.

\subsubsection{Other Influencing Factors}

In addition to patient factors (e.g. obesity, previous history of VTE) there are many other peri- and postoperative factors that influence VTE incidence. For example, a multivariate analysis identified allogenic blood transfusion as a significant risk factor for VTE (associated with a 2.5 times increased VTE risk). ${ }^{83}$ There is also evidence that knee arthroplasty for a joint damaged by rheumatoid arthritis results in less DVT than knee arthroplasty to replace a joint damaged by osteoarthritis; however, this requires further investigation. ${ }^{84}$

Early postoperative mobilisation can reduce relative VTE risk by $65 \%{ }^{85-87}$ Conversely, an increased duration of anaesthesia (3.5+ hours) has been associated with an almost four times increased overall VTE risk in bilateral knee arthroplasty patients (7.5\% vs. 30.2\%). ${ }^{83}$ Notably, bilateral simultaneous knee arthroplasty itself is associated with an increased VTE risk compared to unilateral knee arthroplasty (up to four times increased VTE risk and 80\% increased PE risk), irrespective of time to mobilisation. ${ }^{85,88}$ Interestingly, the type of anaesthesia may also infuence VTE risk: regional anaesthesia has been reported to carry a lower VTE risk than general anaesthesia; consequently it is often recommended in guidelines and commonly used in practice. $^{17,51,89,90}$

A recent study reported that East Asian patients experience very little to no symptomatic VTE following hip and knee arthroplasty, suggesting the value of using routine thromboprophylaxis is debatable in East Asia. ${ }^{81}$ Notably, studies in South East Asia have actually reported higher symptomatic VTE rates to other studies $(5.1 \%){ }^{82}$ Such variations in VTE may be in part due to thromboprophylaxis differences; however there are other influencing factors, some of which are described briefly below.

Patient and surgical factors are not the only elements that can influence VTE risk. The Australian Orthopaedic Association (AOA) has identified that patients operated on by surgeons who conduct a large number of procedures (70+) each year have the best outcome postoperatively. ${ }^{53}$ The difference is influenced by prostheses choice for some, but for others there are other unidentified factors contributing to the observed surgeon variation. ${ }^{53}$

The influence of each type of thromboprophylaxis on VTE incidence is described in the proceeding section; however, the timing of prophylaxis initiation in respect to an operation can also impact VTE risk. For example, evidence suggests thromboprophylaxis should ideally be commenced six to nine hours postoperatively; starting too early (two hours preoperatively, during the perioperative period and up to six hours postoperatively) may increase bleeding and 
haematoma risk, and starting too late ( $>12$ hours postoperatively) appears to increase subsequent thromboembolic complications. ${ }^{91,92}$

Finally, as noted in 2.2.1 Postoperative Venous Thromboemboli, the incidence of VTE extends well beyond hospitalisation, and potentially up to three months after surgery. Consequently, the duration of thromboprophylaxis can influence VTE risk postoperatively. In particular, extending thromboprophylaxis beyond the first fourteen days after surgery can reduce relative VTE risk by approximately 50\% to 70\%, compared to employing thromboprophylaxis for fourteen days or less. ${ }^{51,68,75-78,93}$ 


\subsection{Preventing Venous Thromboemboli}

There are many measures that can be taken to prevent VTE following surgery. These can be grouped by whether they include a medical intervention or not. Those that do not require medical intervention are simple measures such as maintaining adequate hydration; early postoperative mobilisation; promoting healthy postoperative food intake; and encouraging healthy preoperative patient weight or BMI. ${ }^{17,85,86,94-101}$

Measures that involve medical intervention are mechanical prophylaxis and pharmacological prophylaxis; together these strategies tackle the sluggish blood flow and increased clotting factor levels that occur with lower limb surgery. ${ }^{102}$

\subsubsection{Mechanical Prophylaxis}

Healthy lower limb blood flow is maintained by muscle contractions throughout the leg. During walking, pressure on the sole of the foot forces blood to flow out of the span of large veins at the arch of the foot (the plantar venous plexus or PVP) up into the calf, where it is thought to 'prime' the calf muscle (Figure 4). Calf contractions then transport the blood into the deep veins of the thigh, and henceforth, blood flow proceeds to the pelvic veins, vena cava, and ultimately to the heart; all due to lower limb muscle contraction. ${ }^{103,104}$

Figure 4. Emptying of the plantar venous plexus.

a : venous filling of PVP.

$\mathrm{b}$ : weight bearing and subsequent foot arch extension immediately empties plexus.

c : calf contractions transport blood into deep veins of the thigh.

Source: Bergan et al (2006), and Phlebology Journal. ${ }^{103,105}$ a

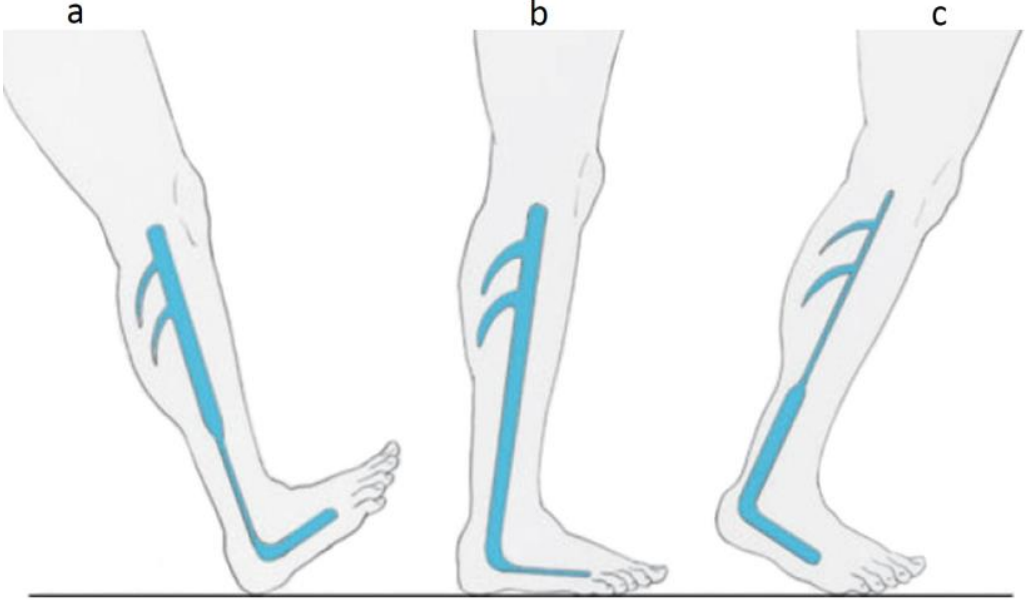

Undoubtedly, a person's regular walking and mobility is limited postoperatively, and therefore so is the regular use of their calf muscles; this can lead to blood stasis and pooling in the veins of the legs. Mechanical thromboprophylaxis aims to combat this by applying physical pressure or stimuli to the relevant structures of the leg.

There are three different types of devices used for mechanical prophylaxis: graduated compression stockings (GCS), intermittent pneumatic compression devices (IPCD) and venous foot pumps (VFP). GCS apply a static circumferential pressure to the lower limb that gradually decreases from the ankle to the thigh. This pressure gradient discourages fluid accumulation and 
promotes venous flow. IPCD sequentially apply varying levels of pressure to the calf by inflating and deflating a compression sleeve to mimic natural calf muscle contractions. ${ }^{106}$ Venous foot pumps are different once again; they apply varying pressures (and sometimes even electrical stimuli) to the bottom of the foot to imitate the physiological pumping action caused by weight bearing on the PVP. ${ }^{106}$

\subsubsection{Safety and Efficacy}

One of the significant advantages of mechanical prophylaxis over pharmacological means is their attractive lack of bleeding potential. This makes them an appealing option for high bleeding risk patients. Another advantage is that they can be used in the contralateral leg perioperatively and in the immediate postoperative period; whereas pharmacological agents are not generally recommended for at least six to eight hours after surgery. ${ }^{68}$ Their use, however, is limited by several logistical and/or compliance issues. For example, they require hospitals to have enough units in good working condition to supply all patients. Furthermore to achieve optimal results they need to be used virtually continuously (including when patients are mobile). ${ }^{68,107-109}$

In practice VFP and IPCD are often only used in hospital because their expense limits the ability to supply all patients with a device at discharge. GCS are significantly cheaper and can even be independently purchased by patients outside of hospital; however, the efficacy of GCS relies heavily on nursing staff selecting the right size and teaching patients to apply them correctly. Choosing the wrong size or incorrect application can result in a significantly increased overall VTE incidence $(25.6 \%$ vs. $6.1 \%, \mathrm{p}<0.05) .{ }^{107}$

Although mechanical prophylaxis strategies have been shown to reduce the risk of VTE, they have been studied with significantly less intensity compared to pharmacological means. Furthermore, it is not always possible to generalise the findings of trials studying the efficacy of each approach. This is because devices are not standardised in their mode of compression, flow rate, or type of sleeve used. ${ }^{110,111}$ Another confounding factor in comparing device studies is that they often include varying adjunctive treatments, including pharmacological prophylaxis. ${ }^{110}$

Nevertheless, a meta-analysis of trials comparing the efficacy of IPCD combined with pharmacological prophylaxis, against either IPCD or pharmacological prophylaxis alone concluded that the combination was up to $80 \%$ more effective than either prophylaxis strategy alone. ${ }^{112}$ Similarly, a meta-analysis of trials exploring VFP use in arthroplasty patients reported them to be up to $40 \%$ more effective than pharmacological prophylaxis; however, results did vary considerably between studies. ${ }^{113}$ The results of trials on GCS have been mixed, potentially due to the pitfalls of incorrect application; in general, however, they appear less effective than other mechanical prophylaxis measures. ${ }^{114}$ 


\subsubsection{Pharmacological prophylaxis}

As previously mentioned, VTE tend to be rich in fibrin and red blood cells. Although the relative proportion of platelets is low, there is evidence to suggest platelet activity is significantly increased perioperatively, thereby augmenting blood coagulability and thrombus formation (Figure 5). ${ }^{57,115,116}$

Figure 5. Clot formation.

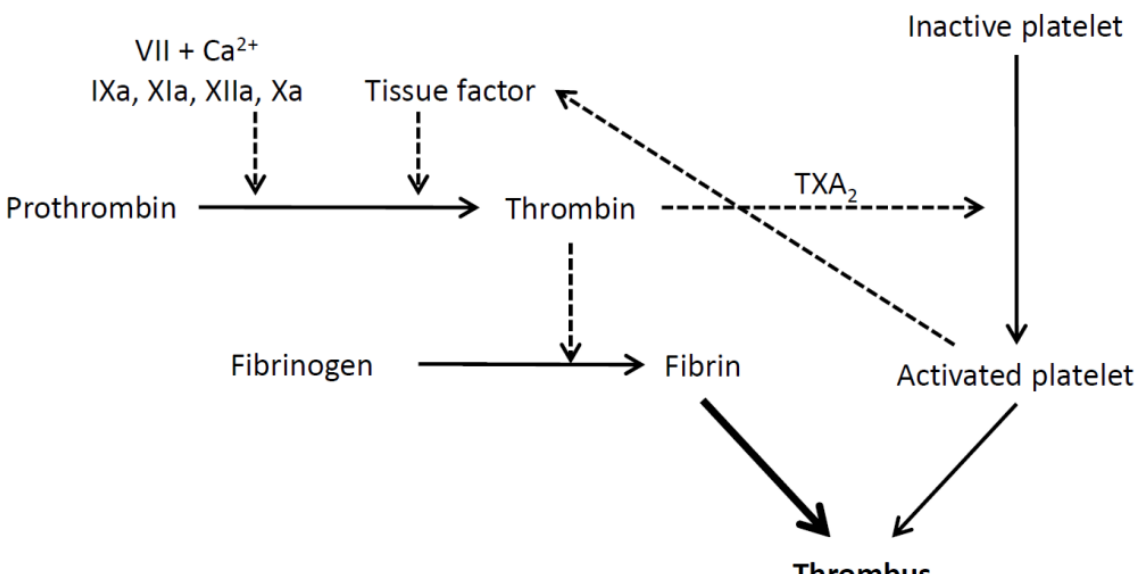

Consequently, there are two classes of pharmacological agents used for thromboprophylaxis postoperatively: anticoagulants and antiplatelets. Anticoagulants prevent blood clots by blocking different steps along the coagulation pathway, thereby limiting thrombin formation. Antiplatelets inhibit platelet activation and aggregation.

\subsubsection{Anticoagulants}

There are a range of anticoagulants recommended for orthopaedic patients, including (in alphabetical order): apixaban, dabigatran, fondaparinux, low molecular weight heparin (LMWH), rivaroxaban, unfractionated heparin (UFH) and warfarin. Each agent blocks a different step(s) in the coagulation pathway (Figure 6). 
Figure 6. Anticoagulants' mode of

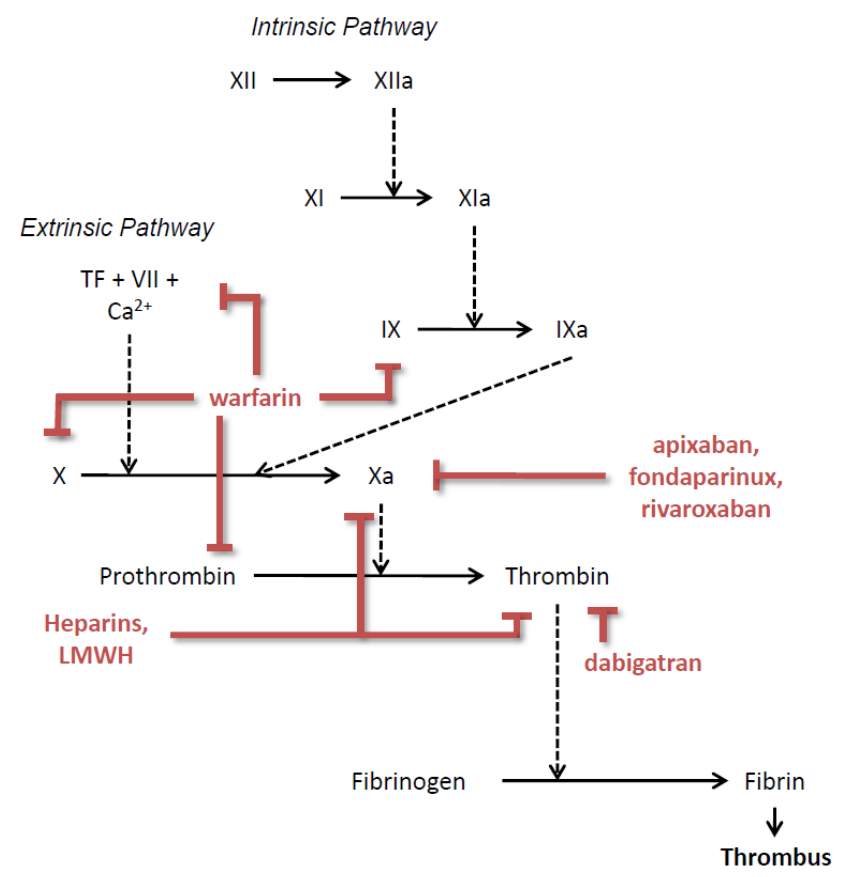

Anticoagulants are administered either by injection (fondaparinux, LMWH and UFH) into the abdomen or orally (apixaban, dabigatran, rivaroxaban and warfarin). Although oral anticoagulants are convenient to administer, apixaban, dabigatran and rivaroxaban were only registered with the Therapeutic Goods Administration in recent years. Consequently, post-marketing surveillance for adverse events is not complete and these novel oral agents are not conventionally recommended as first choice agents postoperatively.

Warfarin is a vitamin $\mathrm{K}$ antagonist (VKA) that has been in use since the 1950s. Despite its long history of use it is also generally not first choice for arthroplasty patients (unless they were using it preoperatively) in Australia due to its significant drug, food and disease interactions, as well as highly individualised and variable dosing. ${ }^{17,119}$ A failure to maintain the appropriate level of anticoagulation with warfarin exposes patients to an increased risk of thrombosis or bleeding. ${ }^{17}$ Furthermore, warfarin has a delayed onset of action and actually induces a procoagulant state during the initiation period. ${ }^{120}$

\subsection{Anticoagulant Safety and Efficacy}

The ability of anticoagulants to prevent VTE is generally accepted; however, their comparative efficacy and safety is unclear. LMWH is the most popular group of agents used in arthroplasty patients in Australia, particularly enoxaparin. ${ }^{121}$ Extended-duration prophylaxis with LMWH can reduce relative VTE risk by $\sim 60 \%$; however, there has often been concern that their subcutaneous administration could potentially reduce patients' overall satisfaction and adherence to treatment, and hence their ability to prevent VTE effectively. ${ }^{75,76,122,123}$ Recent data suggests injection of 
treatment is not a barrier to patient compliance, particularly for injectable anticoagulants that are only administered once daily compared to multiple times a day. ${ }^{124,125}$

Novel oral agents (NOA) on the other hand are convenient to administer and study data suggest they are at least as effective as LMWH against VTE, and in some cases up to $30 \%$ to $60 \%$ more effective. ${ }^{126-129}$ A significant proportion of the arthroplasty associated NOA research to date has been industry supported, and such support may potentially have generated bias in the literature. ${ }^{127,128,130}$ Therefore, more studies are required to confirm the relative efficacy of these agents to LMWH.

In addition to potentially being more effective against VTE, NOA have also been associated with similar as well as statistically significant and non-significant increases in major and minor bleeding, and wound infection post-arthroplasty. ${ }^{127,131,132}$ This is of concern because although UFH, VKA and to some extent LMWH all have well established methods of reversal, there is no antidote or set treatment options for NOA-related emergency situations. ${ }^{131,133,134}$

\subsubsection{Antiplatelets}

Antiplatelets have traditionally been reserved for primary and secondary prevention of cardiovascular disease; however, their use for VTE prevention has increased in popularity in recent times, possibly due to their proposed potential for lower bleeding and faster postoperative recovery. ${ }^{135,136}$ Both commonly used antiplatelets, aspirin and clopidogrel, are oral agents that only require once daily treatment. Clopidogrel inhibits platelet aggregation by inhibiting adenosine diphosphate (ADP)-mediated platelet activation. It also blocks ADP-mediated amplification of platelet aggregation. Aspirin on the other hand blocks the production of thromboxane $A_{2}$, an inducer of platelet aggregation and vasoconstriction. Aspirin is sometimes also used in combination with dipyridamole, another antiplatelet that blocks phosphodiesterase to increase cyclic adenosine monophosphate (Figure 7). 
Figure 7. Mode of platelet activation.

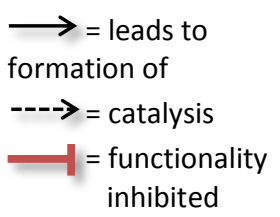

NB: ATP = adenosine triphosphate cAMP = cyclic adenosine monophosphate

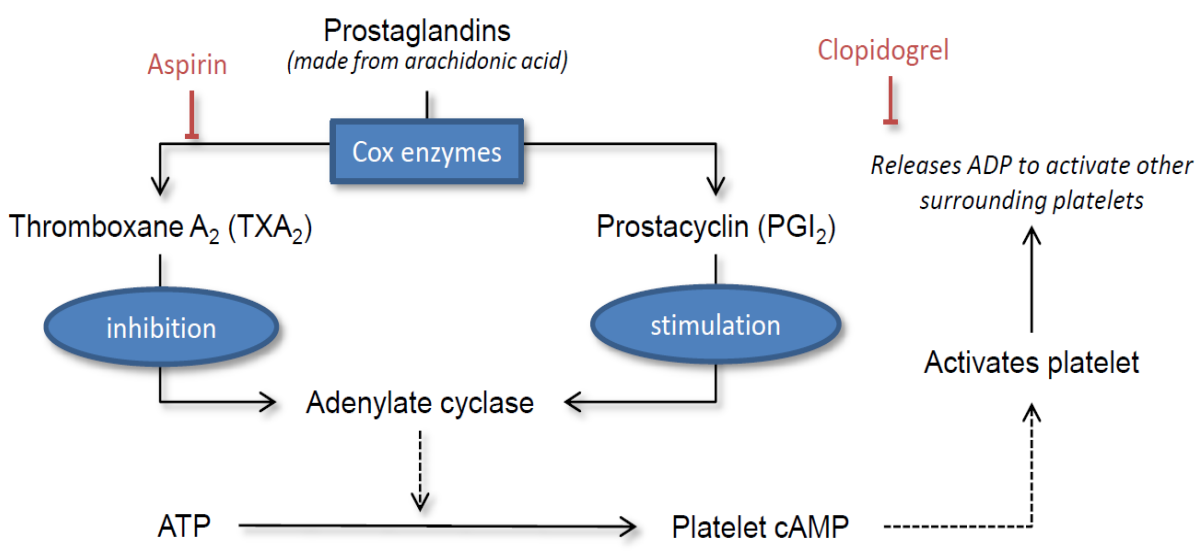

Dipyridamole $\quad \begin{gathered}\text { Phosphodiesterase enzymes } \\ \text { degrade cAMP }\end{gathered}$

Although clopidogrel has been shown to be a more effective antiplatelet agent than aspirin in cardiovascular event prevention, its use is limited by its considerably higher cost and inter-patient variability. ${ }^{137,138}$

\subsection{Antiplatelet Safety and Efficacy}

Studies exploring the ability of aspirin to prevent VTE have reported mixed results. Although the use of antiplatelets for arterial thromboemboli prevention is well established, their use in venous thromboemboli prevention is controversial. ${ }^{139}$ Part of the inconsistency in study findings may be due to a reported variability in aspirin response between patients; however, inter-study methodological differences are likely to also be responsible. ${ }^{140,141}$

Studies that have implemented multimodal prophylaxis post-arthroplasty, which typically consists of regional anaesthesia, early mobilisation, mechanical prophylaxis and aspirin, have reported a symptomatic VTE incidence of $1.9 \%$ to $3.1 \%$ (PE incidence: $0.24 \%$ to $2.6 \%$ ) up to 3 months after surgery. ${ }^{135,142-146}$ These results are encouraging when compared to the estimated three month unprotected VTE incidence of 5.0\%.

Instead of investigating universal aspirin use post-arthroplasty, other studies have implemented protocols that have stratified patients and their thromboprophylaxis according to their VTE risk: low risk patients received aspirin postoperatively; high risk patients received anticoagulants postoperatively. ${ }^{50,147-151}$ These studies have reported comparable VTE rates between the two groups, as have observational studies. ${ }^{152,153}$ To date these findings have been used to claim aspirin is as effective in VTE prevention in low risk patients as anticoagulants are effective in high risk patients; however one could argue that if this was the case, VTE incidence would be significantly lower in the aspirin group as they are inherently a lower risk patient group.

Comparing the direct efficacy and safety of aspirin to anticoagulant agents in any statistically meaningful controlled trial is difficult because the absolute incidence of symptomatic VTE is 
small; to show any statistically significant reduction in PE from $1.0 \%$ to $0.5 \%$, or fatal PE from $0.1 \%$ to $0.05 \%$ would require a sample group of 10,000 or 100,000 patients, respectively. ${ }^{145}$ Nevertheless, Woller et al and Gesell et al have each attempted to compare the two groups headto-head in relatively small study groups. ${ }^{154,155}$

Woller et al compared two groups of patients - those who were treated with aspirin or warfarin depending on their VTE risk (according to American Association of Orthopaedic Surgeons (AAOS) Guideline), and those who received warfarin irrespective of their VTE risk (Figure 8). ${ }^{155,156}$

Figure 8. Graphic representation of patients screened and proportioned to study groups.

NB: $A A O S=$ American Association of Orthopaedic Surgeons; ACCP = American College of Chest Physicians. Source: Woller et al (2012). ${ }^{155}$

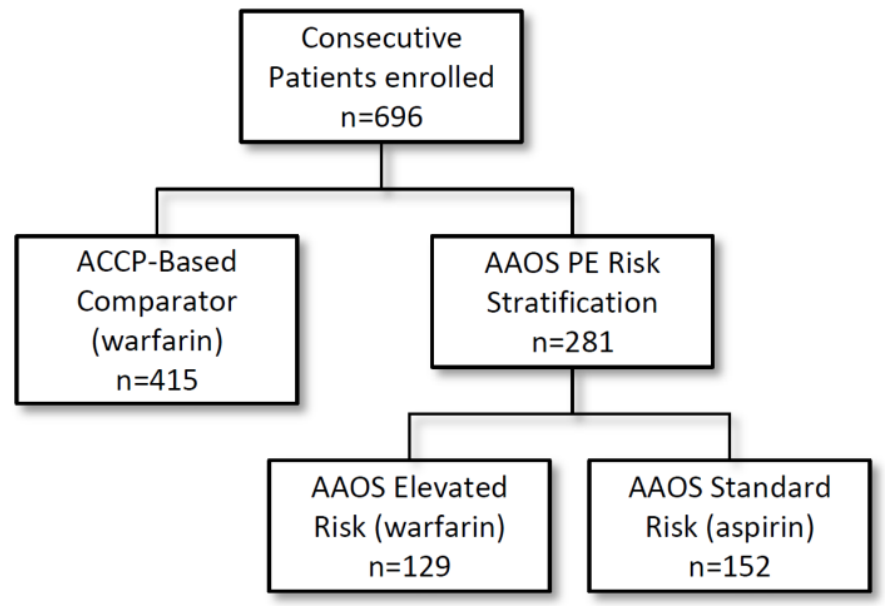

Despite the relatively small numbers enrolled in the study, the AAOS standard risk aspirin group had a statistically significantly higher incidence of symptomatic VTE postoperatively (Table 7).

Table 7. Primary efficacy and safety outcomes. ${ }^{155}$

\begin{tabular}{|c|c|c|c|c|c|c|c|}
\hline \multirow{2}{*}{$\begin{array}{l}\text { Symptomatic } \\
\text { incidence of }\end{array}$} & \multirow{2}{*}{$\begin{array}{l}\text { Comparator } \\
\text { Group } \\
(\mathrm{n}=\mathbf{4 1 5})\end{array}$} & \multicolumn{3}{|c|}{ Elevated Risk Group ( $n=129$ ) } & \multicolumn{3}{|c|}{ Standard Risk Group ( $n=152$ ) } \\
\hline & & Event rate & Odds ratio & $p$ & Event rate & Odds ratio & $p$ \\
\hline PE (\%) & 0.7 & 0 & 0 & 1.0000 & 4.6 & 6.6 & 0.030 \\
\hline DVT (\%) & 0.7 & 0.8 & 1.1 & 1.0000 & 4.6 & 6.6 & 0.030 \\
\hline VTE (\%) & 1.2 & 0.8 & 0.6 & 1.0000 & 7.9 & 7.0 & 0.001 \\
\hline Death (\%) & 1.5 & 0.8 & 0.5 & 1.0000 & 0 & 0 & 0.350 \\
\hline $\begin{array}{l}\text { Major } \\
\text { bleeding (\%) }\end{array}$ & 0.2 & 0 & 0 & 1.0000 & 0.7 & 2.7 & 0.464 \\
\hline
\end{tabular}

NB: $p$ values and odds ratio are compared with comparator group. All VTE were symptomatic.

Conversely, Gesell et al used a similar method (Figure 9) and reported no significant difference in rates of VTE, bleeding or 90-day mortality between the two groups (Table 8). 


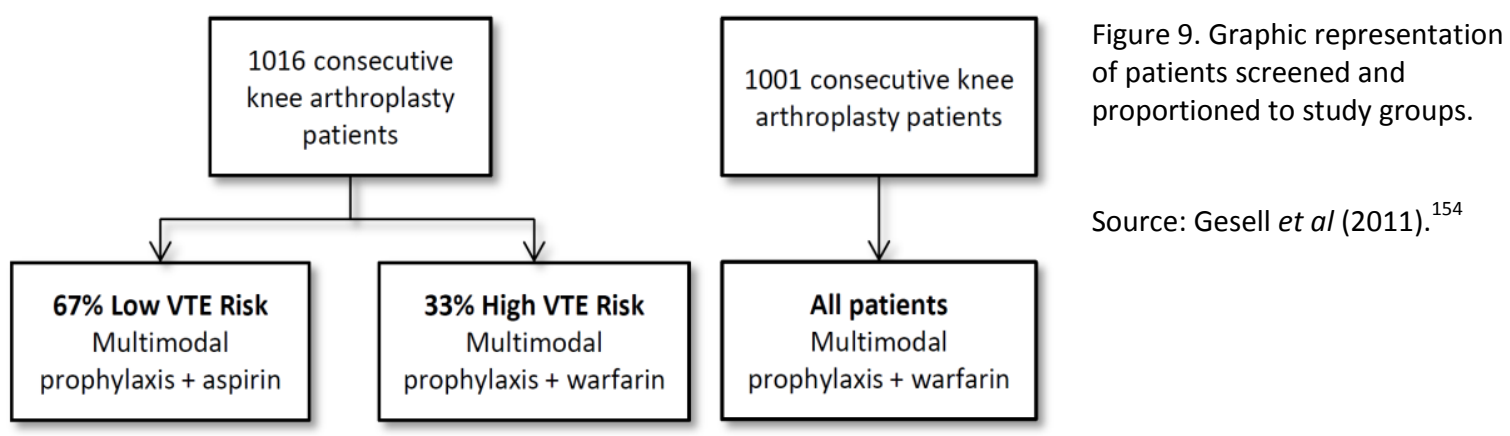

Table 8. Primary efficacy and safety outcomes.$^{154}$

\begin{tabular}{|l|c|c|c|}
\hline Symptomatic incidence of & Study & Control & p \\
\hline \hline PE (\%) & 1.1 & 0.9 & 0.7 \\
\hline DVT (\%) & 0.6 & 1.5 & - \\
\hline VTE (\%) & 2.5 & 2.6 & 0.7 \\
\hline Death (\%) & 0.1 & 0.1 & 0.5 \\
\hline
\end{tabular}

NB: Study group = thromboprophylaxis determined by VTE risk.

It is uncertain why these two studies had such different findings; they used the same aspirin dose postoperatively (325mg twice daily), mobilised patients early postoperatively and employed extended prophylaxis in their patient groups, although Gesell et al did employ a longer duration (Woller et al = 1 month, Gesell et al = 6 weeks). Potentially, the significantly lower VTE incidence in Gesell et al's study could be due to the universal employment of IPCD and epidural anaesthesia in their study (NB: there is no mention of mechanical prophylaxis by Woller et al, and both general and epidural anaesthesia was employed).

The Pulmonary Embolism Prevention (PEP) trial was a prospective multicentre study that is commonly cited in the literature to support the use of aspirin post-arthroplasty. ${ }^{157}$ In this trial Rodgers et al randomised 4,088 patients to receive either aspirin or placebo post-elective hip or knee arthroplasty. The rate of symptomatic VTE was slightly lower in the aspirin group (1.1\% vs. $1.3 \%$ ); however, this did not reach statistical significance. Significantly, 37\% of patients received prophylaxis with LMWH or UFH during the study period, and a further 5\% of patients received non-study aspirin. The authors did not complete a subgroup analysis for these patients to determine what effect anticoagulants had on VTE risk.

The PEP trial also included a second arm that explored VTE risk in hip fracture patients. Similar to the arthroplasty arm, patients were randomised to receive either aspirin or placebo following hip fracture surgery. Once again, many patients received either non-study aspirin (3\%), LMWH (26\%) or UFH (18\%) postoperatively. Furthermore, $8 \%$ of hip fracture trial subjects never started their study treatment. Subgroup analysis was conducted in this arm of the trial (Table 9) and is often used to support aspirin use in arthroplasty patients. 
Table 9. PEP trial subgroup analysis for hip fracture patients.$^{157}$

\begin{tabular}{|l|l|l|}
\hline Thromboprophylaxis & $\mathbf{n}$ & Symptomatic VTE (\%) \\
\hline Nothing & 3789 & $2.6^{\mathrm{a}, \mathrm{b}, \mathrm{c}}$ \\
\hline Aspirin & 3711 & $1.7^{\mathrm{a}}$ \\
\hline LMWH & 1663 & $1.8^{\mathrm{b}}$ \\
\hline LMWH + aspirin & 1761 & $1.3^{\mathrm{c}}$ \\
\hline
\end{tabular}

NB a, b, c indicate statistically significant difference.

Notably, there are limitations and reasons why it is not sound to extrapolate these findings to the arthroplasty arm of the trial. Firstly, the PEP trial was not designed to compare the efficacy of different prophylaxis regimens to prevent VTE. ${ }^{158}$ Its primary aim was to identify what effect aspirin has on the incidence of vascular deaths, non-fatal vascular events and major bleeding complications. ${ }^{159}$ Consequently, Rodgers et al did not collect or analyse VTE risk factors (it is conceivable that anticoagulants were administered in high risk patients), investigate the influence of concomitant mechanical and pharmacological prophylaxis use, or examine the effect of prophylaxis timing (initiation or duration) on prophylaxis efficacy. Furthermore, damaged bone marrow activates the clotting cascade, and a direct positive correlation has been reported between the period of surgery delay after hip fracture trauma and VTE risk. ${ }^{160-163}$ This means that time to surgery and preoperative thromboprophylaxis use should also have been accounted for. Therefore, at best, the PEP trial data indicates that aspirin use may be effective to prevent VTE in some patients (characteristics undefined) undergoing hip fracture surgery.

In summary, the data to date does not support universal aspirin use post-arthroplasty. There may be some merit in using aspirin in very low VTE risk patients; however, further research in this field is required.

\subsubsection{Thromboprophylaxis Recommendations}

Clinical practice guidelines (CPG) have been published by various professional bodies over the last three decades. ${ }^{147}$ Contemporary CPG include those produced by the National Health and Medical Research Council (NHMRC); ${ }^{17}$ the American College of Chest Physicians (ACCP) ${ }^{68,108,164}$ the National Institute of Clinical Excellence (NICE); ${ }^{89}$ the Australia and New Zealand Working Party (ANZWP); ${ }^{1,119,165}$ the American Academy of Orthopaedic Surgeons (AAOS); $;^{156,166}$ and the Arthroplasty Society of Australia (ASA) ${ }^{167}$, a subdivision of the AOA. Prior to the release of the NHMRC Guideline in 2009, the various ACCP guidelines held an arguably prominent spot as the standard of best practice in Australia, and many auditing studies compared prescribing to them. ${ }^{1,8,80,168}$

CPG can be classified as either orthopaedic specific or multidisciplinary depending on whether they contain thromboprophylaxis recommendations for other procedures and conditions, or not. The AAOS and ASA guidelines are orthopaedic specific. The NHMRC, ACCP, NICE, and 
ANZWP guidelines are multidisciplinary guidelines. Historically, the recommendations between these two types of CPG have varied widely as a result of three factors: differences in the approach to collecting and evaluating studies; differences in assumptions made and interpretation of the data; and differences in the relative importance that the panel have ascribed to different postoperative complications, including bleeding and VTE. ${ }^{169}$

For example, in previous editions of their guideline the chest physicians assumed that asymptomatic DVT reductions in trial data would directly correlate to symptomatic VTE reduction. ${ }^{108,164}$ Conversely, orthopaedic surgeons have questioned whether any reductions in symptomatic DVT in trial data has any clinical relevance for symptomatic PE incidence. ${ }^{169,170}$ Furthermore, orthopaedic surgeons have typically been more concerned than the chest physicians have been about the possible consequences of prophylaxis on wound bleeding and joint healing. These differences in assumptions and relative importance ascribed to postoperative complications have resulted in different guideline focuses: the ACCP have generally focussed on preventing venographically proven DVT (the majority of which are asymptomatic), whereas the AAOS have focussed on minimising symptomatic PE. ${ }^{147,156,164,170}$

Despite these methodological and analytical differences, most CPG have endorsed the use of anticoagulants post-arthroplasty, and support the addition of mechanical prophylaxis to anticoagulants. ${ }^{169}$ Furthermore, they have generally agreed on the preferential use of mechanical prophylaxis over anticoagulant use in patients with increased bleeding risk. Last, but not least, CPG generally all recommend against the use of screening techniques (e.g. Doppler or duplex ultrasound) at discharge to screen for asymptomatic VTE. ${ }^{68,166}$

However there have also been four points on which CPG have at times disagreed: the use of aspirin and warfarin as routine prophylaxis agents, the value of using of mechanical prophylaxis by itself, and the ideal duration of postoperative prophylaxis. ${ }^{79,127,139,171}$ Over the years in which the research contained herein was completed, CPG have varied in their stance on these points. Table 10 outlines the thromboprophylaxis recommendations current at the time this research commenced in late 2009. 
Table 10. Thromboprophylaxis recommendations in late 2009.

Recommended agents are highlighted in blue with gradings noted where applicable (e.g. A, 1B; see Appendix A).

\begin{tabular}{|c|c|c|c|c|c|}
\hline Agent & ANZWP $2007^{1}$ & AAOS* $2007^{156}$ & ACCP $2008^{164}$ & NHMRC $2009^{17}$ & ASA - \\
\hline Aspirin & $x$ & B & $x$ & $x$ & \multirow{18}{*}{$\begin{array}{l}\text { To weigh efficacy } \\
\text { against risk of } \\
\text { complications } \\
\text { caused by } \\
\text { preventative } \\
\text { measures. } \\
\text { Mechanical } \\
\text { thromboprophylaxi } \\
\text { s strongly } \\
\text { recommended. Use } \\
\text { of aspirin, warfarin, } \\
\text { UFH, LWMH, } \\
\text { fondaparinux and } \\
\text { the melagatrans } \\
\text { and duration of } \\
\text { these agents to be } \\
\text { carefully } \\
\text { considered and } \\
\text { thoroughly } \\
\text { discussed with the } \\
\text { patient. }\end{array}$} \\
\hline Dabigatran & & & & B & \\
\hline Fondaparinux & NG & B & $1 \mathrm{~A}$ & B & \\
\hline LMWH & NG & B & $1 \mathrm{~A}$ & $A$ & \\
\hline Rivaroxaban & & & & B & \\
\hline UFH & & & & & \\
\hline VKA & $x / \checkmark$ & B & $1 \mathrm{~A}$ & $x / \checkmark$ & \\
\hline Duration & 28 to 35 days & $\begin{array}{l}7 \text { to } 42 \text { days } \\
\text { (varies dep. on } \\
\text { agent) (C) }\end{array}$ & $\begin{array}{l}\text { Min. } 10 \text { days, } \\
\text { up to } 35 \text { days }\end{array}$ & Up to 35 days & \\
\hline $\begin{array}{l}\text { Mechanical } \\
\text { Prophylaxis }\end{array}$ & $\begin{array}{l}\text { AND IPCD \&/or } \\
\text { GCS }\end{array}$ & $\begin{array}{l}\text { To be used until } \\
\text { discharged } \\
\text { home }\end{array}$ & - & $\begin{array}{l}\text { GCS, IPCD, or VFP, } \\
\text { until fully mobile }\end{array}$ & \\
\hline Aspirin & $x$ & B & $x$ & $x$ & \\
\hline Dabigatran & & & & B & \\
\hline Fondaparinux & NG & B & $1 \mathrm{~A}$ & B & \\
\hline LMWH & NG & B & $1 \mathrm{~A}$ & A & \\
\hline Rivaroxaban & & & & B & \\
\hline UFH & & & & & \\
\hline VKA & $x / \checkmark$ & B & $1 \mathrm{~A}$ & $x / \checkmark$ & \\
\hline Duration & 5 to 10 days & $\begin{array}{l}7 \text { to } 42 \text { days } \\
\text { (varies dep. on } \\
\text { agent) (C) }\end{array}$ & $\begin{array}{l}\text { Min. } 10 \text { days, } \\
\text { up to } 35 \text { days }\end{array}$ & Up to 14 days & \\
\hline $\begin{array}{l}\text { Mechanical } \\
\text { Prophylaxis }\end{array}$ & $\begin{array}{c}\text { AND IPCD \&/or } \\
\text { GCS }\end{array}$ & $\begin{array}{l}\text { To be used until } \\
\text { discharged } \\
\text { home }\end{array}$ & $\begin{array}{l}\text { OR Optimal } \\
\text { IPCD (1A) }\end{array}$ & $\begin{array}{l}\text { GCS, IPCD, or VFP, } \\
\text { until fully mobile }\end{array}$ & \\
\hline
\end{tabular}

*AAOS recommendations vary based on patient risk of PE and major bleeds.

NB: NG = no grading; $x=$ recommendation against use; $x / \checkmark=$ only recommended under certain conditions;

Min. $=$ minimum.

In the last three years the ASA, ACCP, NICE and AAOS each released a new edition of their guideline (Table 11). 
Table 11. Updated AAOS, ACCP and ASA thromboprophylaxis recommendations.

Recommended agents are highlighted in blue with gradings noted where applicable (e.g. A, 1B; see Appendix A).

\begin{tabular}{|c|c|c|c|c|}
\hline Agent & ASA $2010^{167}$ & ACCP $2012^{68}$ & NICE $2010^{89}$ & AAOS $2011^{166}$ \\
\hline Apixaban & & 1B & & \multirow{9}{*}{$\begin{array}{l}\text { 'We suggest the use of } \\
\text { pharmacologic agents and/or } \\
\text { mechanical compressive devices } \\
\text { for the prevention of VTE in patient } \\
\text { undergoing elective hip or knee } \\
\text { arthroplasty and who are not at } \\
\text { elevated risk beyond that of the } \\
\text { surgery itself for VTE or bleeding. }\end{array}$} \\
\hline Aspirin & NG & $1 \mathrm{~B}$ & & \\
\hline Dabigatran & & $1 \mathrm{~B}$ & NG & \\
\hline Fondaparinux & & 1B & NG & \\
\hline 吾 LMWH & NG & $1 B^{*}$ & NG & \\
\hline 은 Rivaroxaban & NG & $1 \mathrm{~B}$ & NG & \\
\hline UFH & & $1 \mathrm{~B}$ & NG & \\
\hline 음 VKA & NG & $1 \mathrm{~B}$ & & \\
\hline Duration & $\begin{array}{l}3 \text { to } 6 \\
\text { weeks }\end{array}$ & $\begin{array}{c}\text { Min. } 10-14 \\
\text { days, up to } 35 \\
\text { days }\end{array}$ & 28 to 35 days & \\
\hline $\begin{array}{l}\text { Mechanical } \\
\text { Prophylaxis }\end{array}$ & IPCD, VFP & & $\begin{array}{l}\text { GCS, VFP or IPCD } \\
\text { until mobile }\end{array}$ & \multirow{11}{*}{$\begin{array}{l}\text { In the absence of reliable evidence } \\
\text { about how long to employ these } \\
\text { prophylactic strategies, it is the } \\
\text { opinion of this work group that } \\
\text { patients and physicians discuss the } \\
\text { duration of prophylaxis.' }\end{array}$} \\
\hline Apixaban & & $1 \mathrm{~B}$ & & \\
\hline Aspirin & NG & $1 B$ & & \\
\hline Dabigatran & & $1 B$ & NG & \\
\hline Fondaparinux & & $1 B$ & NG & \\
\hline 吾 LMWH & NG & $1 B^{*}$ & NG & \\
\hline 을 Rivaroxaban & NG & $1 \mathrm{~B}$ & NG & \\
\hline$\frac{E}{c}$ UFH & & $1 B$ & NG & \\
\hline 竞 VKA & NG & $1 B$ & & \\
\hline Duration & $\begin{array}{l}3 \text { to } 6 \\
\text { weeks }\end{array}$ & $\begin{array}{c}\text { Min. } 10-14 \\
\text { days, up to } 35 \\
\text { days }\end{array}$ & 10 to 14 days & \\
\hline $\begin{array}{l}\text { Mechanical } \\
\text { Prophylaxis }\end{array}$ & IPCD, VFP & & $\begin{array}{l}\text { GCS, VFP or IPCD } \\
\text { until mobile }\end{array}$ & \\
\hline
\end{tabular}

*ACCP 2012 Guidelines recommend LMWH in preference to the other agents (Grade 2C/2B).

NB: NG = recommendation is not graded. Min. = minimum.

\subsubsection{Other Recommendations}

In addition to recommending pharmacological and mechanical thromboprophylaxis, the NHMRC, ANZWP, AAOS and NICE guidelines all recommend early mobilisation to prevent VTE. 1,17,89,166 Furthermore, the NHMRC, ANZWP and NICE guidelines also recommend adequate hydration as a simple measure that should be applied as standard practice to prevent VTE. 


\subsection{Guideline Adherence}

Despite the wide promulgation of CPG, studies report that thromboprophylaxis is underutilised both in Australia and internationally. ${ }^{4-11,172}$ In 2006/7 an international audit of 32 countries determined that anywhere between $0 \%$ and $100 \%$ of at risk orthopaedic patients received ACCP approved agents postoperatively. ${ }^{11}$ Almost $95 \%$ of the Australian patients involved in the audit received ACCP approved agents. Similarly, a retrospective review conducted in a New South Wales hospital found that $87 \%$ of patients received ACCP approved agents at the recommended dose following hip and knee arthroplasty. ${ }^{80}$

However, not all Australian data demonstrates such high adherence to contemporary guidelines. A study conducted in a Western Australian hospital reported that thromboprophylaxis was initiated in less than $50 \%$ of patients who underwent hip arthroplasty. ${ }^{9}$ Similar practices were described in another Western Australian study, where $51.5 \%$ of at risk patients received ACCP recommended thromboprophylaxis; only $18.9 \%$ of patients continued to receive prophylaxis after discharge. ${ }^{8}$ These studies indicate that there is a high degree of variability in thromboprophylaxis prescribing in Australian hospitals. ${ }^{5,8,98}$ This has been identified by the National Institute of Clinical Studies in Australia (NICS) and the NHMRC as a significant evidence-practice gap. ${ }^{10,173}$

Grol et al identified that guidelines with clear noncontroversial and evidence based recommendations are more likely to be adhered to than guidelines with recommendations that are not clear, are controversial, or are based on opinion. ${ }^{20}$ What is evident when reviewing the updated recommendations (Table 11) is that the AAOS and ACCP have reduced the grading strength and become broader in their recommendations for postoperative thromboprophylaxis. This indicates a level of general uncertainty as to what constitutes best practice. It is not surprising then that there is a high degree of variability in adherence rates to thromboprophylaxis guidelines both nationally and internationally.

In 2009 when the NHMRC Guideline was released, the most recently published Australian guideline adherence study reported data from 2005; more recent studies have used internal benchmarks instead of guidelines in their audits. ${ }^{174}$ Excluding publications from the data contained in this thesis, there have not been any further Australian papers published to date describing thromboprophylaxis prescribing patterns and/or guideline adherence in Australia.

\subsubsection{Implementing Guidelines}

CPG can be limited in their ability to affect physician behaviour, and the implementation of CPG into practice requires more than simply publishing them. ${ }^{59,175}$ There are many strategies that can be employed to increase the uptake of a CPG into practice, and multifaceted methods are often recommended and implemented effectively. ${ }^{176,177}$ Straus et al note that an implementation strategy that includes an analysis of factors that influence prescribing, as well as a selection of appropriate 
and feasible knowledge translation interventions and indicators to measure impact, increases the likelihood of success. ${ }^{178}$

Factors that can influence adherence to CPG can do so both positively and negatively. Those factors that positively impact on adherence are termed enablers. Those factors that negatively impact on adherence are termed barriers. Barriers to the uptake of CPG can be broadly categorised into three types: those in which physician knowledge imposes a barrier, those in which physician attitude imposes a barrier and, finally, those in which external factors prevent guideline adoption (Figure 10). ${ }^{179,175}$

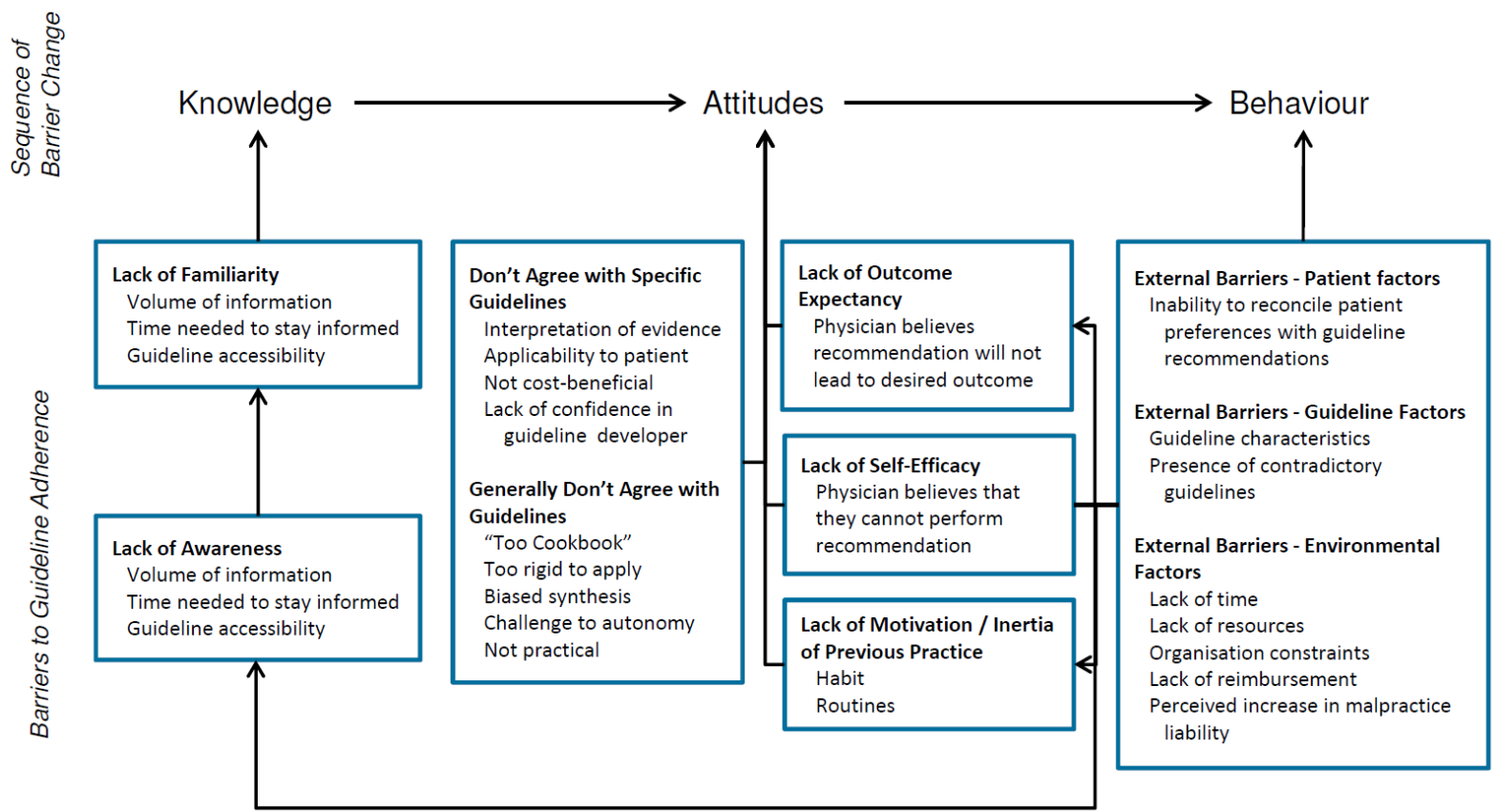

Figure 10. Barriers to physician adherence to CPG.

Source: Cabana et al (1999). ${ }^{175}$

It is in the best interest of both hospitals and patients for evidence-based CPG to be effectively implemented. In 2013 Duff et al reported that a 20\% improvement in adherence to an internal hospital thromboprophylaxis protocol was estimated to have resulted in 13 fewer deaths, 84 fewer symptomatic DVTs, 19 fewer symptomatic PEs, and 512 fewer hospital-bed days. ${ }^{179}$ These improvements were associated with an overall cost saving of $\$ 245,429$ over 12 months.

Although guideline adherence has been documented to be low in Australia and overseas, enablers and barriers to thromboprophylaxis guideline uptake and adherence by orthopaedic surgeons have not been analysed in the literature. ${ }^{180}$ Nevertheless, in 2011 Duff et al reported that the following factors were identified as barriers to thromboprophylaxis guideline uptake in a metropolitan Sydney hospital: A lack of motivation to change, a lack of systems support, a knowledge or awareness deficit and disputed evidence. ${ }^{177}$ The following two sections summarises 
known enablers and barriers to thromboprophylaxis prescribing (as a surrogate to guideline adherence) following orthopaedic surgery overseas.

\subsubsection{Enablers}

In 1992 Rodgers et al surveyed 94 New Zealand (NZ) orthopaedic surgeons to identify their thromboprophylaxis prescribing preferences, and factors that might increase the likelihood of them prescribing thromboprophylaxis. ${ }^{181}$ A patient history of VTE was identified as the most important factor that would increase likelihood of prescribing thromboprophylaxis (Figure 11). Moreover, gross obesity, prolonged preoperative immobility and active malignancy were also identified as important patient factors by approximately $50 \%$ of surveyed surgeons.

Figure 11. Factors that increase the likelihood of prescribing pharmacological prophylaxis (1992).

Factors of $\square$ great importance, and $\square$ little importance, as indicated by survey participants.

Source: Rodgers et al $(1994)(\mathrm{n}=94){ }^{181}$

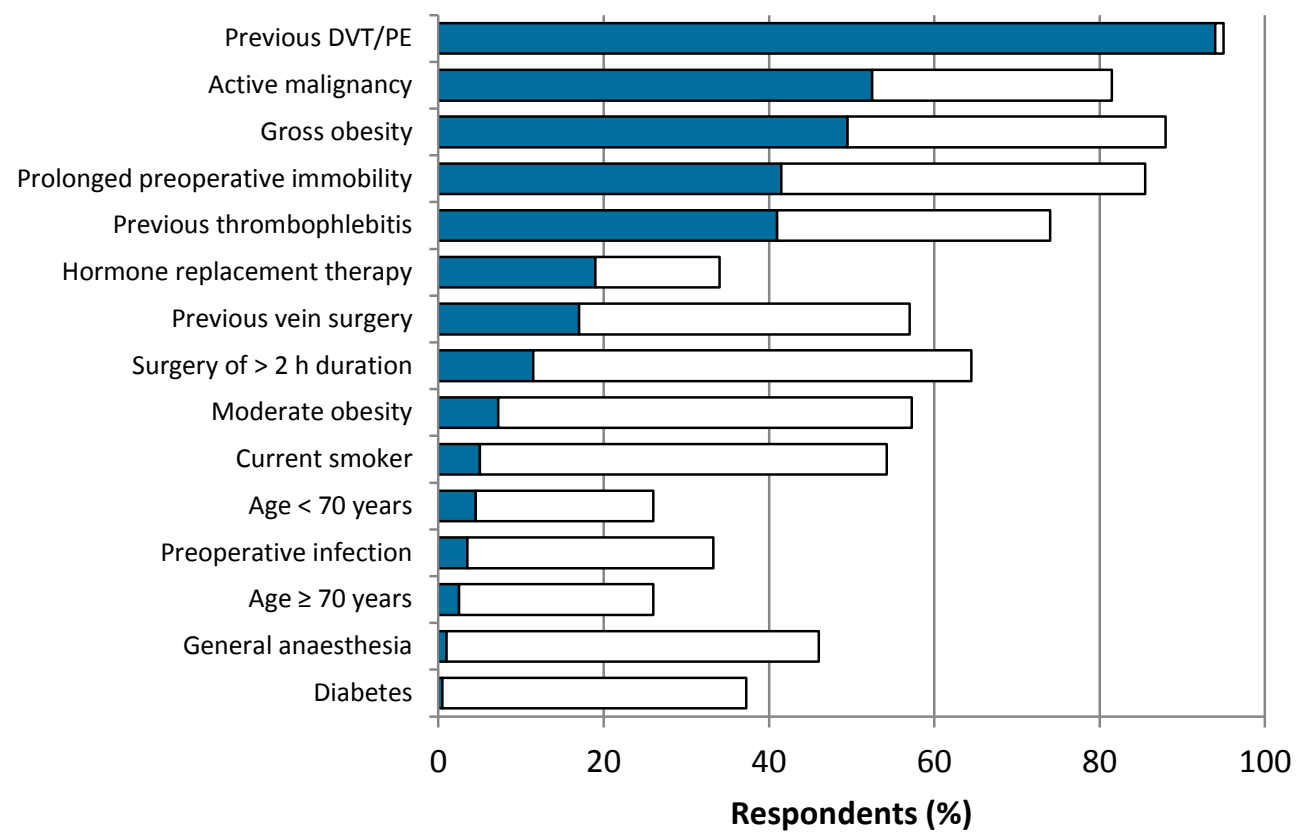

The survey was repeated seven years later in 1999 by Walker et al. At this point it was identified that orthopaedic surgeons had substantially changed their prescribing of pharmacological prophylaxis, with a significant increase in the use of anticoagulants, and in particular LMWH (from 55\% to 76\%). ${ }^{182}$ It was suggested by Walker et al that the increase in LMWH use was likely due to 'emerging evidence for the use of pharmacological thromboprophylaxis, medical education, the impact of a new cohort of surgeons, awareness of thromboprophylaxis [possibly as a result of the Pulmonary Embolism Prevention (PEP) Trial that was conducted between 1992 and 1998 and involved many NZ orthopaedic surgeons], increasing concern over litigation, and intensive marketing by pharmaceutical companies'. ${ }^{182}$ Interestingly, the top three factors that surgeons identified as being of great importance in increasing the likelihood of them prescribing 
thromboprophylaxis remained the same: previous VTE, active malignancy and gross obesity (Figure 12).

Figure 12. Factors that increase the likelihood of prescribing pharmacological agents (1999).

Factors of $\square$ great importance, and $\square$ little importance, as indicated by survey participants.

Source: Walker et al (2002) $(n=123) .^{182}$

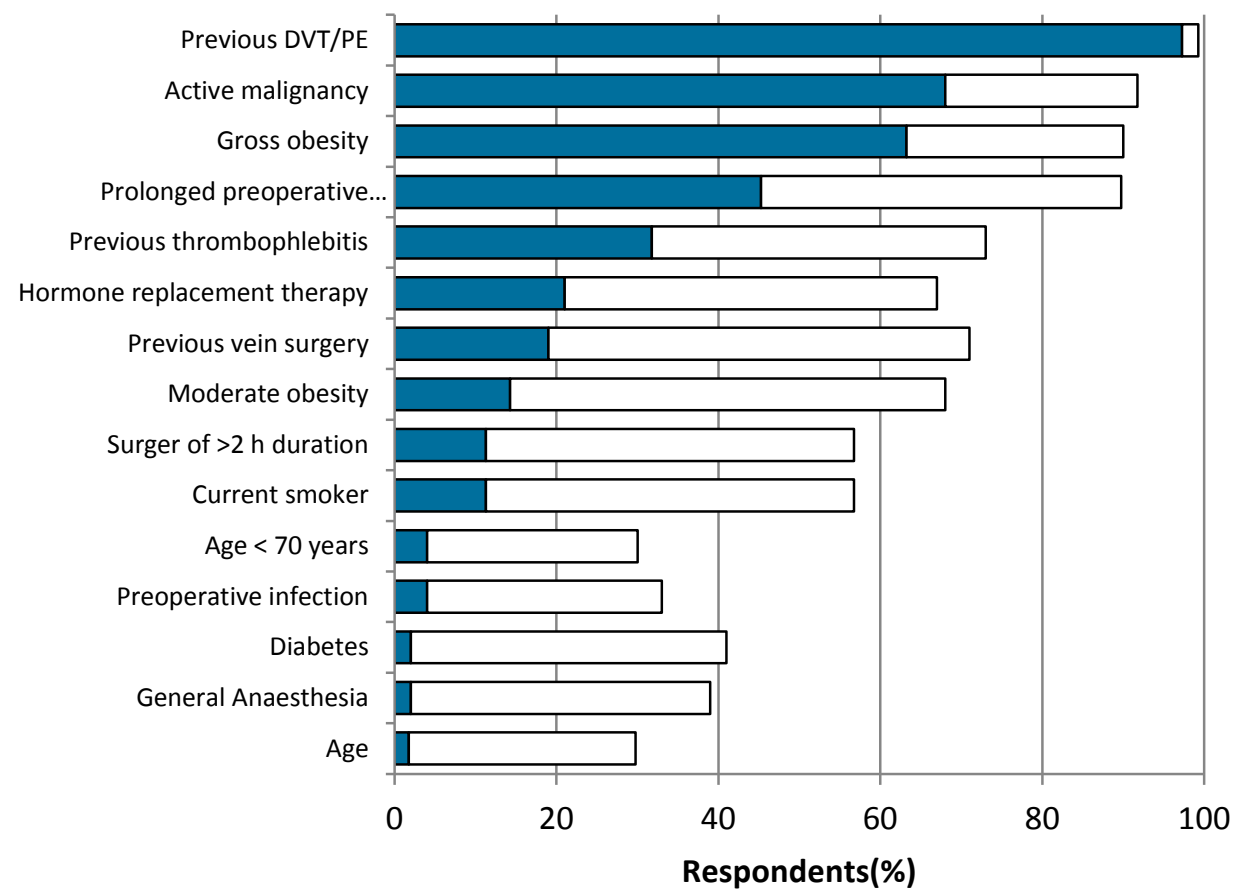

More recently, a survey of Malaysian orthopaedic surgeons by Zairul-Nizam et al identified that a surgical procedure's risk grading was the most significant factor that influenced thromboprophylaxis prescribing postoperatively, followed by a patient history of previous VTE, the presence of concurrent illnesses and patient obesity (Figure 13) ${ }^{183} \mathrm{NB}$ : It is uncertain what year the survey itself was conducted (corresponding author did not reply to query), however the study was published in 2003 .

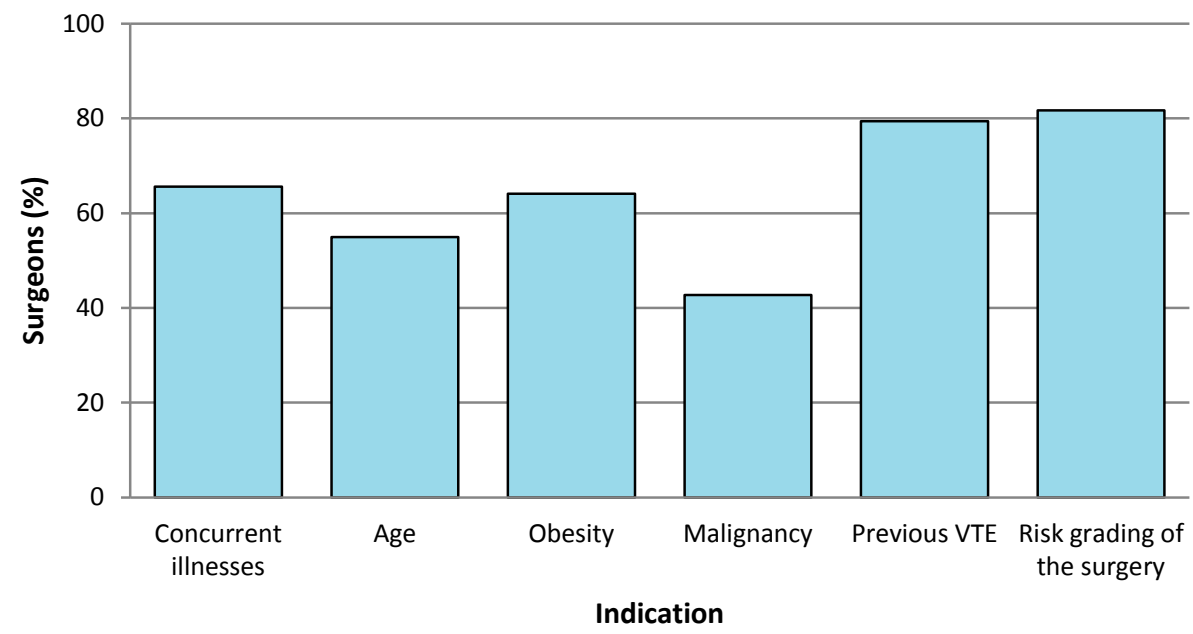

Figure 13. Indications for thromboprophylaxis use $(n=131)$.

Source: Zairul-Nizam et al (2003). ${ }^{183}$ 


\subsubsection{Barriers}

In addition to surveying factors that would increase the likelihood of prescribing thromboprophylaxis, these studies explored barriers to thromboprophylaxis prescribing. Rodgers et al reported that NZ surgeons surveyed in 1992 identified major bleeding diathesis and active peptic ulcers as the top two patient factors that would stop them prescribing pharmacological agents postoperatively (Figure 14). ${ }^{181}$

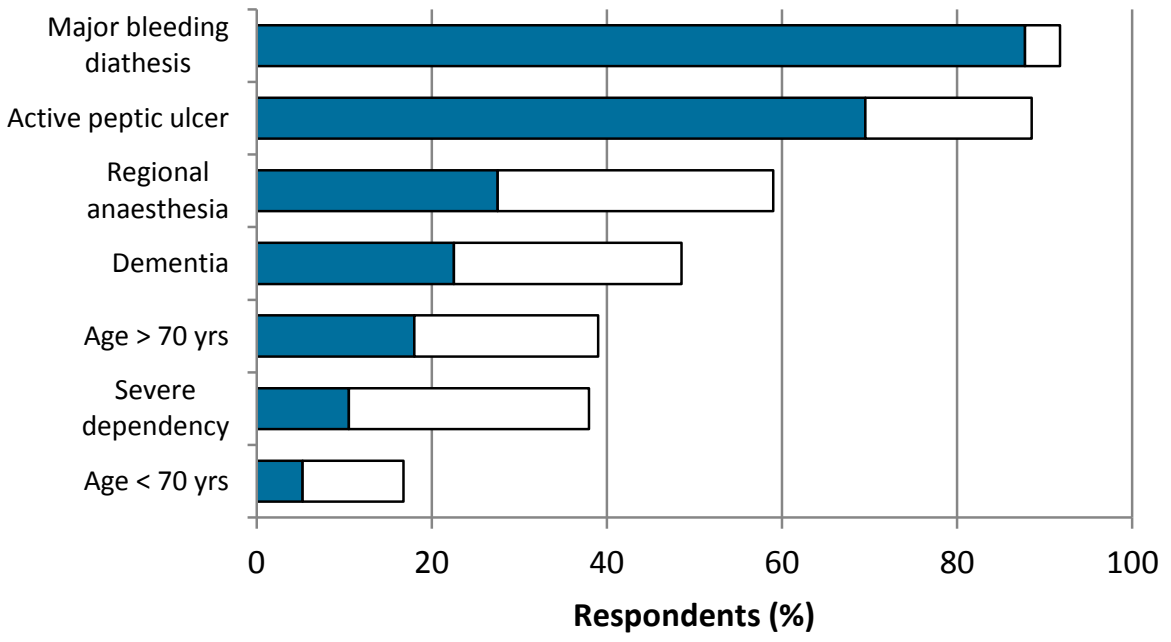

Figure 14. Factors that might decrease the use of pharmacological prophylaxis.

Factors of $\square$ great importance, and $\square$ little importance, as indicated by survey participants.

Source: Rodgers et al $(1994)(n=94) .{ }^{181}$

Furthermore, Rodgers et al reported that more than one third of surgeons cited fear of bleeding complications and a low perceived VTE risk as barriers to prescribing pharmacological thromboprophylaxis postoperatively (Figure 15). ${ }^{181}$ Similar findings were reported by Walker et $a l .{ }^{182}$ A perception that VTE risk is too low to warrant prophylaxis is not surprising given the median time to a symptomatic VTE following surgery is 17 to 30 days and the vast majority of VTE are asymptomatic and/or occur post-discharge. ${ }^{184,185}$ Moreover, the length of hospital stay has shortened over the years. As previously mentioned, some patients stay in hospital as little as 2 days after surgery, and it is not uncommon for patients to be discharged before regaining full mobility, thereby increasing their risk of VTE. ${ }^{186}$ These factors can mask the true incidence of VTE and can accordingly become barriers to the optimal use of thromboprophylaxis. 


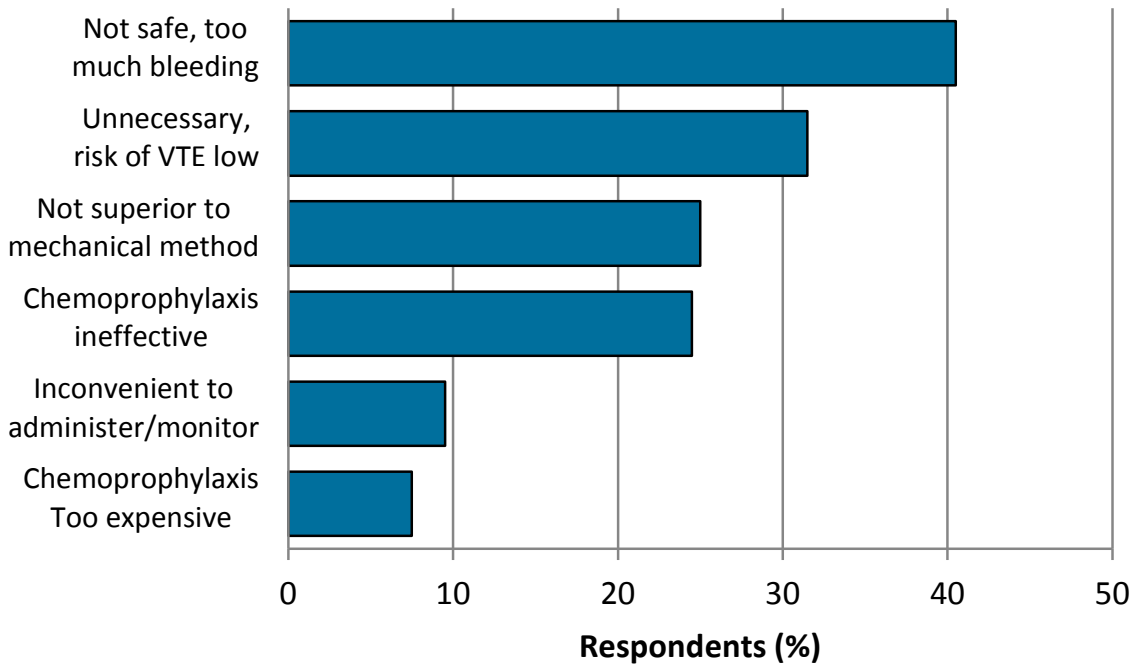

Figure 15. Barriers to pharmacological thromboprophylaxis use.

Surgeons were asked to indicate whether their prophylaxis use was limited by all or some of these factors.

Source: Rodgers et al $(1994)(n=123) .{ }^{181}$

Zairul-Nizam et al also identified bleeding concerns as a major barrier to prescribing pharmacological agents, as well as a perceived low risk of VTE postoperatively (Figure 16). ${ }^{183}$ Concerns about patient adherence, bleeding, adverse effects and outpatient monitoring associated with pharmacological agents have also been reported in other literature. ${ }^{55,56}$

Figure 16. Reasons for not instituting pharmacological prophylaxis.

NB: 'Religious' refers to the religious practice of the patient e.g. Muslim patients who decline the use of porcine based agents.

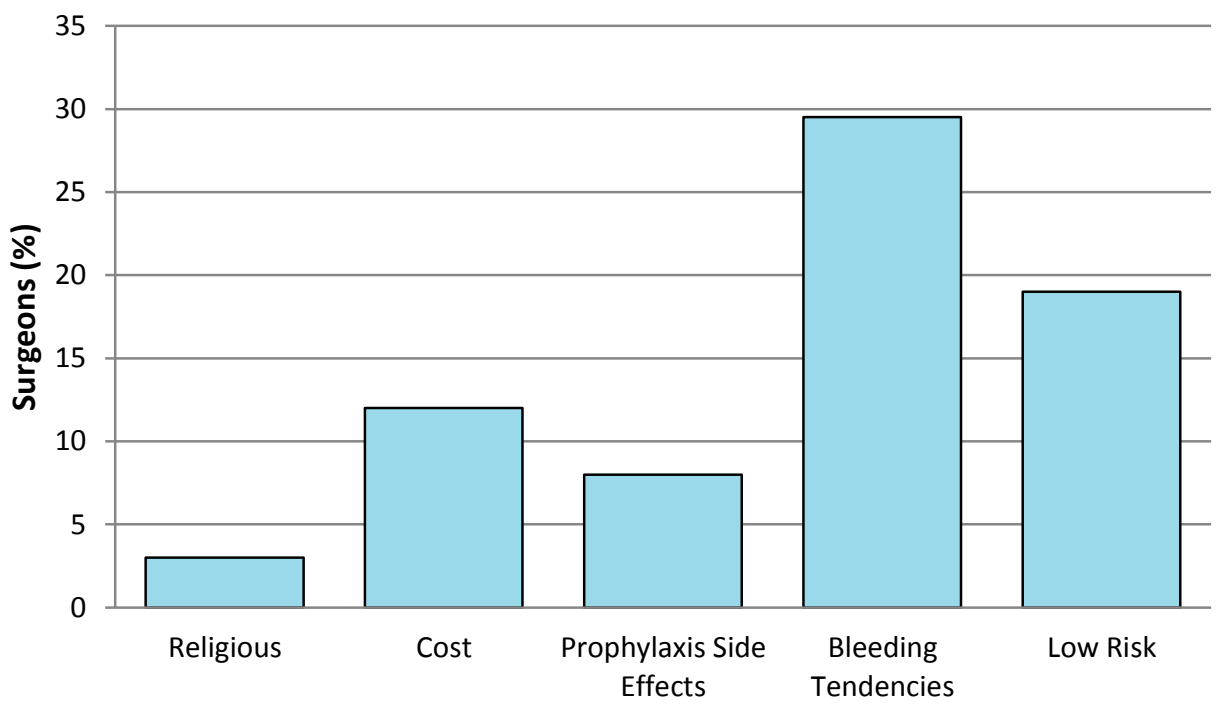

Source: Zairul-

Nizam et al

Reason $(2003)(n=131){ }^{183}$

At the commencement of this research in 2009 there had been no studies conducted in Australia analysing factors that influence thromboprophylaxis prescribing specifically postarthroplasty, and the data aforementioned dated back to the 1990s, and early 2000s (assuming Zairul-Nizam et al conducted their survey in early 2000s, just prior to publishing in 2003). 
In February 2012 Molnar et al published their findings of a survey conducted in 2009 of Australian orthopaedic surgeons conducting hip and knee arthroplasties $(n=581) .{ }^{121}$ The survey identified that $98 \%$ of participants regularly prescribed pharmacological thromboprophylaxis following arthroplasty, particularly favouring anticoagulants. Interestingly, these surgeons were more likely to prescribe anticoagulants in fear of litigation $(19.2 \%$ vs. $10.1 \%)$, and were more likely to rely on protocols or guidelines (32.2\% vs. $17.2 \%$ ), instead of basing their decision on their own reading (52.4\% vs. $71.3 \%$ ). No further enablers or barriers to prescribing were reported, leaving enablers and barriers to prescribing thromboprophylaxis post-arthroplasty in Australia largely unknown.

\subsection{Rationale for Research}

Approximately $3 \%$ to $5 \%$ of arthroplasty patients will experience a symptomatic VTE following their surgery, roughly equating to 2,500 to 4,150 Australians each year. Many more will have no telling symptoms. The real burden of these clots is undetermined; however, they can be significant and at times even fatal. Although thromboprophylaxis has been reported to prevent up to $60 \%$ of VTE following arthroplasty, and guidelines have been published for years, anecdotal evidence suggested local thromboprophylaxis prescribing was persistently suboptimal at the commencement of this research. Therefore, the research contained herein commenced with a review of local thromboprophylaxis prescribing in 2009. 


\section{Chapter Three: Local ThromboprophylaXis Prescribing}

\subsection{Introduction}

The Royal Hobart Hospital (RHH) is Tasmania's leading hospital, servicing a population of $\sim 240,000$ people. As the major teaching and research hospital in the state it had approximately 550 beds (including 90 day-only beds). The Orthopaedic Unit had 25 dedicated adult beds in the department.

\subsection{Aims}

The aims of this study were two-fold:

- to identify and compare local thromboprophylaxis prescribing patterns with contemporary recommendations, and

- to measure the incidence of symptomatic VTE within 90 days of surgery.

\subsection{Methods}

\subsubsection{Patient Selection}

The medical records of 300 consecutive patients who underwent a hip or knee arthroplasty (150 of each) between June 2007 and June 2009 were retrieved and systematically reviewed. Where patients had multiple arthroplasty admissions within the review period only the most recent admission was included.

\subsubsection{Data Collection}

Data extraction included information on age, gender, operation, VTE risk factors, mode of anaesthesia, kidney function, mechanical and pharmacological prophylaxis (including duration and dosage), hospital length of stay and reported postoperative bleeding complications. Clinically symptomatic VTE, including those causing readmission or presentation to a general practitioner or emergency department within 90 days of surgery, were also recorded for all patients. Symptomatic VTE was defined as the presence of symptoms confirmed by a positive duplex ultrasonography for DVT and positive pulmonary CT angiography for PE. 


\subsubsection{Thromboprophylaxis Analysis}

Pharmacological agents available during the study period at the RHH were (in alphabetical order) aspirin \pm dipyridamole, clopidogrel, fondaparinux, LMWH, unfractionated heparin and warfarin. GCS were the only mechanical options available.

In this study, pharmacological prophylaxis prescribing was compared with recommendations made in the ACCP $7^{\text {th }}$ Ed. Guideline. ${ }^{108}$ The ANZWP $3^{\text {rd }}$ Ed. Guideline was also available to surgeons during the study period and the ACCP $8^{\text {th }}$ Ed. Guideline was released half-way through the study period; both had similar recommendations to the ACCP $7^{\text {th }}$ Ed. Guideline (Table 12).

Table 12. Guideline recommendations during the study period.

Recommended agents are highlighted in blue with gradings noted (e.g. A, 1B; see Appendix A).

\begin{tabular}{|c|c|c|c|}
\hline Agent & ANZWP $3^{\text {rd }}$ Ed. $^{1}$ & ACCP $7^{\text {th }}$ Ed. $^{108}$ & ACCP $8^{\text {th }}$ Ed. $^{164}$ \\
\hline Aspirin & $x$ & $x$ & $x$ \\
\hline Fondaparinux & NG & $1 \mathrm{~A}$ & $1 \mathrm{~A}$ \\
\hline LMWH & NG & $1 \mathrm{~A}$ & $1 \mathrm{~A}$ \\
\hline Rivaroxaban & & & \\
\hline VKA & & $1 \mathrm{~A}$ & $1 \mathrm{~A}$ \\
\hline Duration & $28-35$ days & $\begin{array}{l}\text { Min. } 10 \text { days, and } \\
\text { up to } 28 \text { to } 35 \text { days }\end{array}$ & $\begin{array}{c}\text { Min. } 10 \text { days, up to } 35 \\
\text { days }\end{array}$ \\
\hline Mechanical & IPCD \&/or GCS & - & - \\
\hline Aspirin & $x$ & $x$ & $x$ \\
\hline Fondaparinux & NG & $1 \mathrm{~A}$ & $1 \mathrm{~A}$ \\
\hline LMWH & NG & $1 \mathrm{~A}$ & $1 \mathrm{~A}$ \\
\hline Rivaroxaban & & & \\
\hline VKA & & $1 \mathrm{~A}$ & $1 \mathrm{~A}$ \\
\hline Duration & $5-10$ days & Min. 10 days & $\begin{array}{c}\text { Min. } 10 \text { days, up to } 35 \\
\text { days }\end{array}$ \\
\hline Mechanical & IPCD \&/or GCS & $\underline{\mathrm{OR}}$ IPCD (1A) & $\underline{\mathrm{OR}}$ IPCD (1A) \\
\hline
\end{tabular}

NB: NG = no grading; $x=$ recommendation against agent; $-=$ recommend against use as sole form of thromboprophylaxis; $\min =$ minimum.

Patients were deemed as having received ACCP recommended thromboprophylaxis if the agent, dose and duration of therapy prescribed was in line with the ACCP $7^{\text {th }}$ Ed. Guideline. To assess the suitability of the pharmacological agents and doses prescribed for each patient, renal function was estimated using the Cockroft-Gault equation:

$$
\mathrm{CrCl}=\frac{(140-\text { age }) \times \mathrm{IBW}}{0.814 \times \mathrm{SeCr}}
$$

Where $\quad \mathrm{CrCl}=$ creatinine clearance

$\mathrm{IBW}=$ ideal body weight $(\mathrm{kg})$, and

$\mathrm{SeCr}=$ serum creatinine $(\mu \mathrm{mol} / \mathrm{L})$.

NB: Ideal body weight was estimated by entering each patient's height and gender into an electronic ideal body weight calculator produced by Therapeutic Guidelines Ltd. ${ }^{187}$ 
As 'duration of therapy' was vague for hip arthroplasty patients (up to 35 days), the parameter was defined as $\geq 28$ days, in line with the ANZWP Guideline. ${ }^{1}$

\subsubsection{Exclusion criteria}

Contraindications to mechanical thromboprophylaxis included severe cellulitis, leg deformity and severe dermatitis. Pharmacological agents were deemed appropriate for patients unless any of the following relative and absolute contraindications were recorded in their medical notes: bleeding disorder (e.g. haemophilia), recent central nervous system bleeding, intracranial or spinal lesion, abnormal blood coagulation, thrombocytopenia or severe platelet dysfunction, severe hepatic disease (including oesophageal varices), severe renal dysfunction (creatinine clearance $<10 \mathrm{~mL} / \mathrm{min}$ ) or pregnancy. When active bleeding events reviewed by medical staff in the hospital were judged to be critical enough to warrant cessation of pharmacological prophylaxis the patient was recorded as inappropriate for receiving further pharmacological prophylaxis.

\subsubsection{Statistics}

All information was stored in an Access database (Microsoft ${ }^{\circledR}$ Access 2010, Washington, USA), and analysed using the statistical program Statistical Package for the Social Sciences (SPSS) 19.0 (IBM ${ }^{\circledR}$ Armonk, NY USA). The differences between groups were tested using the t-test for independence for continuous data, and the chi square test for categorical variables. Pearson's rank correlation coefficients were calculated for measuring correlations. Continuous variables were summarised as means with standard deviation (SD) and ranges where appropriate. Only $\mathrm{p} \leq 0.05$ values were considered statistically significant.

\subsection{Results}

\subsubsection{Patient Demographics and Anaesthesia}

In total, 300 patients (169 females, 131 males) with a mean age of 68.7 years (SD 10.4) were included in the study. The mean length of stay in hospital was 6.9 days (SD 3.6) following surgery. General anaesthesia was employed in most patients, either alone $(185,63.3 \%, n=292)$ or in combination with neuraxial anaesthesia $(48,16.4 \%)$. 
Table 13. Patient demographics (150 HA and KA each, $\mathrm{N}=300)$.

\begin{tabular}{|l|l|l|l|l|l|l|l|l|l|}
\hline & \multicolumn{3}{|c|}{ HA } & \multicolumn{3}{c|}{ KA } & \multicolumn{3}{|c|}{ Overall } \\
\hline mean/n & N & $\%$ / SD & mean /n & N & $\% /$ SD & mean /n & $\% /$ SD \\
\hline Female gender & 68.9 & 150 & 10.7 & 68.5 & 150 & 10.0 & 68.7 & 10.4 \\
\hline Body mass index* & 28.0 & 150 & $56.0 \%$ & 85 & 150 & $56.7 \%$ & 169 & $56.3 \%$ \\
\hline Length of stay & 7.2 & 131 & 5.5 & 32.5 & 142 & 6.3 & 30.3 & 6.3 \\
\hline CrCl & 65.4 & 140 & 4.3 & 6.6 & 150 & 2.7 & 6.9 & 3.6 \\
\hline $\begin{array}{l}\text { Anticoagulant use } \\
\text { prior to admission }\end{array}$ & 10 & 150 & $6.7 \%$ & 9 & 62.1 & 145 & 1.9 & 63.7 & 24.5 \\
\hline $\begin{array}{l}\text { Antiplatelet use prior } \\
\text { to admission }\end{array}$ & 48 & 150 & $32.0 \%$ & 53 & 150 & $6.0 \%$ & 19 & $6.3 \%$ \\
\hline Discharged home & 112 & 149 & $75.2 \%$ & 120 & 149 & $80.5 \%$ & 232 & $77.9 \%$ \\
\hline
\end{tabular}

NB: Excludes patients who developed a complications postoperatively, and those with contraindications to pharmacological prophylaxis.

* indicates statistically significant difference.

\subsubsection{Contraindications to Thromboprophylaxis}

There were no absolute or relative contraindications to mechanical or pharmacological thromboprophylaxis documented in patients' histories. Therefore, for the purpose of this study all patients were deemed appropriate for receiving both forms of thromboprophylaxis postoperatively.

\subsubsection{Mechanical Thromboprophylaxis}

Only 42 patients $(14.0 \%)$ had documented GCS use. The mean BMI of these patients was significantly lower than those not prescribed GCS ( 28.3 vs. $30.7 \mathrm{~kg} / \mathrm{m}^{2}, \mathrm{p}<0.05, \mathrm{n}=273$ ). There was no significant difference in the demographics of patients who received GCS and those who did not. No patients received documented GCS at discharge. Obese patients (BMI $\geq 25.0)$ were less likely to be prescribed mechanical prophylaxis than non-obese patients (13.1\% vs. $17.8 \%$ respectively), however this did not reach statistical significance.

\subsubsection{Pharmacological Thromboprophylaxis}

\subsubsection{Agent}

Inpatient and discharge pharmacological thromboprophylaxis data was available for 298 patients (99.3\%). Of these patients, most $(99.0 \%)$ received pharmacological prophylaxis postoperatively during the inpatient phase which, in the absence of complications, was continued throughout their admission. Anticoagulants were most commonly prescribed, particularly LMWH (Table 14). Over a quarter of patients $(82,27.7 \%)$ were prescribed an anticoagulant and an antiplatelet concurrently during their inpatient stay (Table 14). 
Excluding patients who developed a VTE or bleeding event during their admission $(n=4)$, only 108 patients $(36.5 \%, \mathrm{n}=296)$ continued to receive any pharmacological prophylaxis following discharge. The majority of these patients received an antiplatelet (Table 14).

\begin{tabular}{|c|c|c|c|c|}
\hline \multirow[b]{2}{*}{ Agent } & \multicolumn{2}{|c|}{ Inpatient } & \multicolumn{2}{|c|}{ Discharge } \\
\hline & $\mathrm{n}$ & $\%$ & $\mathrm{n}$ & $\%$ \\
\hline LMWH & 173 & 58.4 & 21 & 7.1 \\
\hline LMWH + antiplatelet & 72 & 24.3 & 9 & 3.0 \\
\hline Fondaparinux & 28 & 9.5 & 2 & 0.7 \\
\hline Fondaparinux + antiplatelet & 9 & 3.0 & 2 & 0.7 \\
\hline Warfarin & 8 & 2.7 & 12 & 4.1 \\
\hline Warfarin + antiplatelet & - & - & 1 & 0.3 \\
\hline No agent & 3 & 1.0 & 186 & 62.8 \\
\hline Heparin & 1 & 0.3 & - & - \\
\hline Heparin + antiplatelet & 1 & 0.3 & 1 & 0.3 \\
\hline Antiplatelet only & 1 & 0.3 & 60 & 20.3 \\
\hline Unknown & - & - & 2 & 0.7 \\
\hline
\end{tabular}

NB: Excludes patients who developed a bleed (2) or VTE (2) postoperatively whilst still in hospital.

Most discharge anticoagulants were newly initiated in hospital $(39,81.2 \%)$, particularly LMWH (87.2\%) and fondaparinux (10.2\%). Patients were significantly more likely to be discharged with an anticoagulant if they were transferred to a rehabilitation unit compared to going home $(63.3 \%$ vs. $5.2 \%, \mathrm{p}<0.001)$.

Most patients discharged on an antiplatelet $(51,85.0 \%)$ were taking their antiplatelet agent prior to surgery, and only $15.0 \%$ were newly initiated in hospital. Aspirin was the most commonly prescribed antiplatelet.

\subsubsection{Duration}

The mean duration of thromboprophylaxis prescribed was 6.0 days (SD 2.7). There was no significant difference in the number of days prescribed for hip or knee arthroplasty patients (6.0 days for both), however patients who were discharged to a rehabilitation unit were prescribed slightly longer thromboprophylaxis courses (6.9 days vs. 5.9 days, $\mathrm{p}=0.01$ ), particularly in hip arthroplasty patients ( 7.1 vs. 5.8 days, $\mathrm{p}=0.03$ ).

Excluding patients who developed an inpatient clot $(n=1)$ or were using an anticoagulant at home $(n=5)$, one knee arthroplasty patient was discharged home on an anticoagulant agent $(0.9 \%$, $\mathrm{N}=114)$. Excluding patients who developed a bleed $(\mathrm{n}=2)$ or were using an anticoagulant at home $(\mathrm{n}=5)$, and patients whose discharge thromboprophylaxis was unknown $(\mathrm{n}=2)$, only nine patients were discharged home on an anticoagulant agent $(8.7 \%, \mathrm{~N}=103)$. 


\subsubsection{Contemporary Guidelines}

Kidney function and thromboprophylaxis dose and duration data was not available for all patients, however where possible, patients' thromboprophylaxis was compared to the contemporary guideline to assess suitability of prescribing. Excluding patients prescribed therapeutic anticoagulation for pre-existing medical conditions, and patients who experienced a bleed or VTE during their inpatient admission, only $8.8 \%$ of knee patients $(10, n=113)$ and $1.0 \%$ of hip patients $(1, n=103)$ discharged home received ACCP recommended prophylaxis (including agent, dose and duration). This equates to $5.1 \%$ overall $(11, \mathrm{n}=216)$. The remaining patients either received no pharmacological prophylaxis or received it at an inappropriate dose and/or duration (Figure 17).

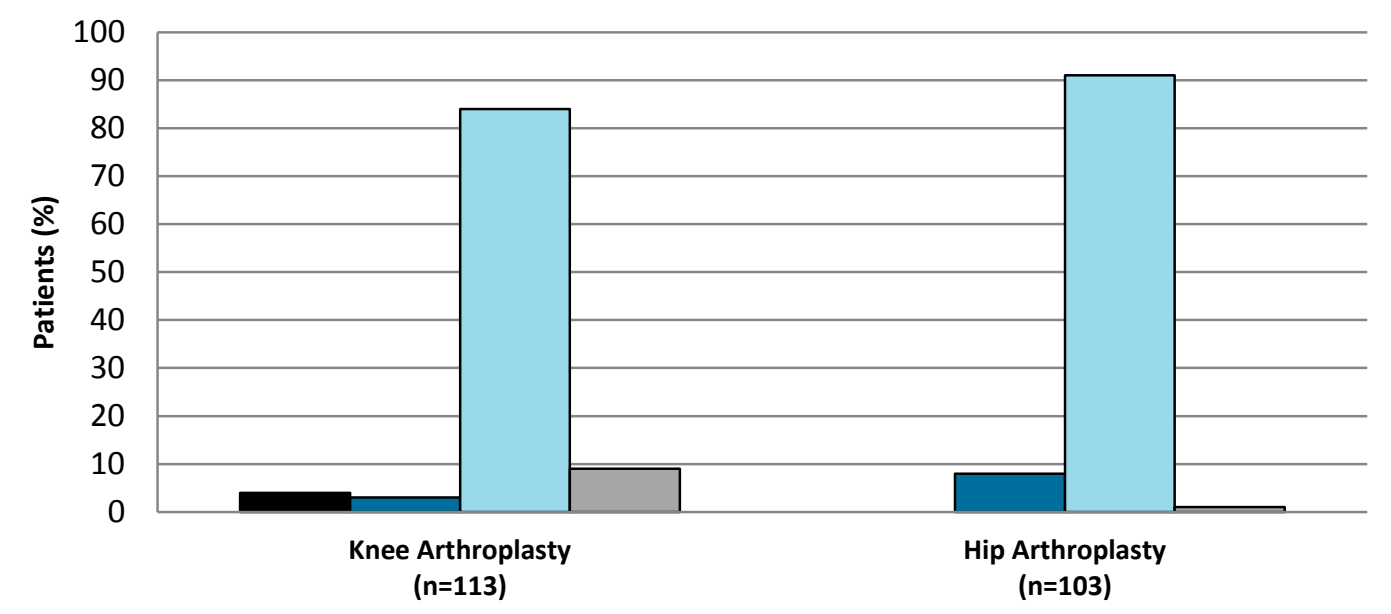

Figure 17. Proportion discharged home with ACCP recommended prophylaxis $\quad(\mathrm{N}=216)$.

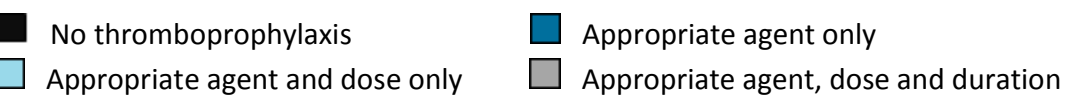

Obese patients (BMI $\geq 25.0$ ) were more likely to receive ACCP appropriate pharmacological prophylaxis compared to non-obese patients (5.6\% vs. $2.7 \%$ ); however this did not reach statistical significance.

\subsubsection{Postoperative Complications}

\subsubsection{Bleeding}

Two inpatients $(0.6 \%)$ developed a bleed postoperatively that warranted cessation of their pharmacological prophylaxis; both were male hip arthroplasty patients with either liver or renal impairment.

\subsubsection{Symptomatic VTE}

The 90-day incidence of symptomatic VTE was $2.7 \%$ (95\% CI: $1.0-5.0 \%$, Table 15). The incidence in knee patients was 4.0\% (95\% CI: 1.0-8.0\%; 4 DVTs, 1 PE and 1 DVT + PE). The incidence in hip patients was 1.3\% (95\% CI: 0-5.0\%; 2 DVTs). The in-hospital VTE incidence was 
$0.7 \%(2, n=300)$ and occurred at 4 and 5 days following surgery. Both patients were prescribed anticoagulants at the time their $\operatorname{clot}(\mathrm{s})$ occurred.

\begin{tabular}{|c|c|c|c|c|}
\hline \multirow[b]{2}{*}{ VTE } & \multicolumn{2}{|c|}{ HA } & \multicolumn{2}{|c|}{ KA } \\
\hline & $\mathrm{n}$ & $\%$ & $\mathrm{n}$ & $\%$ \\
\hline Overall $(n=300)$ & 2 & 1.3 & 6 & 4.0 \\
\hline In hospital $(n=300)$ & - & - & 2 & 1.3 \\
\hline DVT & - & - & 1 & 0.7 \\
\hline $\mathrm{DVT}+\mathrm{PE}$ & - & - & 1 & 0.7 \\
\hline After discharge $(n=296)^{* *}$ & 2 & 1.4 & 4 & 2.7 \\
\hline PE & - & - & 1 & 0.7 \\
\hline DVT & 2 & 1.4 & 3 & 2.0 \\
\hline
\end{tabular}

NB: **Excludes patients who developed a clot or bleed in hospital (i.e. $\mathrm{HA}=148$ patients, $\mathrm{KA}=148$ patients).

Excluding inpatient clots and bleeds, VTE occurred in $2 \%$ of patients following discharge (5 DVTs, 1 PE, n=296). The median time to VTE outside of hospital was 11 days (range: 7 to 31 days). Four patients were readmitted to the hospital for a median of 2.5 days to receive VTE treatment under a medical team (range 1 to 6 days). Of the patients who suffered a thromboembolic event following discharge, only one patient had been prescribed an anticoagulant at discharge. This patient had been using warfarin prior to surgery and was discharged with warfarin and LMWH to cover until the International Normalised Ratio (INR) was therapeutic. It is uncertain if the INR was therapeutic at the time of the event, six days following discharge, however sub therapeutic INR at the time of discharge has been associated with both a higher readmission rate, and risk of complications ${ }^{188}$. The remaining five patients who developed VTE following discharge did not receive ACCP recommended prophylaxis, predominantly due to a shortfall in the duration of therapy prescribed (average of only 5.2 days prescribed, SD 1.5). No significant risk factors were identified for developing VTE following surgery.

\subsection{Discussion}

In-hospital anticoagulant use was routine and despite bleeding risk concerns, over $25 \%$ of patients received both an antiplatelet and anticoagulant agent during their admission. ${ }^{139}$ Notably, this study determined that over $60 \%$ of patients did not receive any documented thromboprophylaxis on discharge from hospital. When comparing prescribing practices to the ACCP $7^{\text {th }}$ Ed. Guideline, only $5.1 \%$ of patients discharged home received complete courses of pharmacological prophylaxis overall. This was predominantly due to a shortfall in the duration of therapy prescribed. This has previously been described in a retrospective observational studies. ${ }^{155,172}$ 
There was a trend towards knee arthroplasty patients receiving full prophylaxis courses more often than hip arthroplasty patients (8.8\% vs. $1.0 \%)$, likely because the average length of stay in hospital (6.9 days SD 3.6) was considerably closer to the recommended duration of therapy for knee patients compared to hip patients (10 vs. 28 days minimum). This meant that hip patients were more reliant on anticoagulant prophylaxis being prescribed for them after discharge from the ward. This only occurred in nine hip patients discharged home $(8.7 \%, \mathrm{n}=103)$ and typically for a much shorter duration than was required to complete the recommended 28 days of treatment. In the strictest sense, the ACCP $7^{\text {th }}$ Ed. Guideline recommends a minimum of 10 days thromboprophylaxis, with extension up to 28 to 35 days following hip arthroplasty (Table 12). Notably, using this parameter to define adherence (10 to 35 days) does not significantly alter the assessed adherence level to the ACCP $7^{\text {th }}$ Ed. Guideline (6.7\%), because the average duration of anticoagulant therapy for hip patients was only 6.0 days (SD 2.7).

This study identified an in-hospital and 90-day VTE incidence of $0.7 \%$ and $2.0 \%$, respectively, comparable with other studies in the literature. ${ }^{62,184}$ The majority of VTE occurred following discharge, which has also been previously reported in the literature. ${ }^{51,62,143,184,185}$ As all readmissions to hospital for VTE treatment were under medical units, local VTE incidence following arthroplasty may be underestimated by surgical teams.

As previously noted in Table 2, patient obesity increases VTE risk. ${ }^{2,97-99,189,190}$ It is surprising then that obese patients (BMI $\geq 25.0$ ) were less likely to be prescribed mechanical prophylaxis than non-obese patients in this study. This may be due to difficulties that can arise from fitting stockings on obese patients. Interestingly, White et al reported mechanical prophylaxis to be effective in nonobese hip arthroplasty patients, not obese patients. ${ }^{87}$ Sadeghi et al report that standard doses of pharmacological agents are effective regardless of BMI; obese patients in the present study tended to be more likely to receive ACCP appropriate pharmacological prophylaxis compared to nonobese patients. ${ }^{85}$

There were several limitations to this study. It was retrospective in nature and relied solely on documentation in hospital records to identify thromboprophylaxis use, possible contraindications, and thrombotic and bleeding events. The retrospective nature of the study also introduced potential low-prescribing bias following hospital discharge, as although there were no specific recommendations on discharge summaries, it is possible that general practitioners may have independently prescribed thromboprophylaxis for their patients after discharge. Similarly, the retrospective nature may have posed potential bias for a low reported VTE incidence as not all cases of out-of-hospital diagnosed and treated VTE may have presented to the RHH. That the RHH is the only public hospital within the area should have minimised this potential limitation, although it is feasible that some patients may have been admitted to one of several private hospitals in the area, or treated directly by their general practitioner with no feedback to the RHH. 
The retrospective nature of the study did not allow exploration of potential barriers to routine thromboprophylaxis use, including perceived and actual risk of major bleeding associated with pharmacological prophylaxis. Being a retrospective study, it was not possible to determine if such perceived risks affected thromboprophylaxis prescribing, particularly at discharge.

Despite these limitations, the findings indicate that the lack of consensus as to what constituted appropriate thromboprophylaxis following hip and knee arthroplasty during the study period left most patients without guideline recommended thromboprophylaxis, potentially leaving many patients exposed to a higher risk of VTE. ${ }^{155}$ 


\section{Chapter Four: Prescribing in Australian Public Hospitals}

\subsection{Introduction}

The retrospective review conducted at the RHH confirmed the existence of a guideline-practice gap. A retrospective audit of thromboprophylaxis prescribing was undertaken in several public hospitals to identify if this finding was representative of prescribing around Tasmania and interstate. All of Tasmania's public hospitals were approached, and several extra were chosen from two states with relatively easy flight access from Tasmania: Victoria and New South Wales.

\subsection{Aim}

The aim of this study was to identify what thromboprophylaxis prescribing practices prevail at public hospitals in Tasmania, Victoria and New South Wales, and compare them with the NHMRC Guideline.

\subsection{Methods}

\subsubsection{Hospital Selection}

Fifteen public hospitals listed on the Australian Orthopaedic Association's National Joint Registry were approached to participate in this study. These hospitals included all of Tasmania's public hospitals as well as hospitals chosen from the National Joint Registry list of statistics contributors that were relatively easy to access for a visiting researcher (in case outside data collection was required). Twelve hospitals agreed, however most could not complete the study: two hospitals' orthopaedic departments vetoed the project due to its auditing nature; one hospital had participated in an intervention study to increase thromboprophylaxis prescribing during the study period (which would have biased the data); one hospital lost the resources needed to support the project; and two hospitals simply stopped responding to correspondence. Ultimately only six hospitals completed the audit: three in Tasmania (including the RHH), two in Victoria and one in New South Wales.

\subsubsection{Data Collection}

The medical records of the first 30 consecutive patients who underwent a hip or knee arthroplasty (15 of each) in 2011 were retrieved and systematically reviewed. This sample size allowed for a descriptive snapshot of prescribing at each hospital, whilst also ensuring the numbers were manageable enough to allow for audits at multiple hospitals. Where patients had multiple 
arthroplasty admissions within the review period only the most recent admission was included. Data extraction followed the same process outlined in 3.3.2 Data Collection. Unfortunately data was missing from some patient files/datasets, including inpatient complications, dosages, creatinine clearance information and total duration of therapy prescribed. This was taken into account in the analysis and is a major limitation of this study.

\subsubsection{Thromboprophylaxis Analysis}

The NHMRC Guideline was released nationally over 12 months prior to the study period. Patients were deemed as having received NHMRC recommended thromboprophylaxis if the agent, dose and duration of therapy prescribed was in line with the Guideline. To assess the suitability of the pharmacological agents and doses prescribed for each patient, renal function was estimated using the Cockroft-Gault equation (see page 33).

As the 'duration of therapy' recommended in the NHMRC Guideline was deemed vague (up to 14 days for knee arthroplasty patients, and up to 35 days for hip arthroplasty patients), the following duration parameters were used in this study's analysis to define adherent prophylaxis: a minimum of 10 to 14 days for knee patients and a minimum of 28 to 35 days for hip patients. These parameters are aligned with those used in the previous study (Chapter 3).

Any VTE, bleeding (including wound ooze) or infective complications that occurred during the inpatient period (either on the orthopaedic or rehab unit) were recorded and included in the final analysis.

\subsubsection{Statistics}

All information was stored in an Access database (Microsoft ${ }^{\circledR}$ Access 2010), and analysed using the statistical program SPSS 20. The differences between groups were tested using the t-test for independence for continuous data, and the chi square test for categorical variables. Continuous variables were summarised as means with standard deviation. Only $\mathrm{p} \leq 0.05$ values were considered statistically significant.

\subsection{Results}

\subsubsection{Patient Demographics and Anaesthesia}

In total, 180 patients (108 females, 70 males) with a mean age of 68.1 years (SD 10.4) were included in the study (Table 16). The mean length of stay on the orthopaedic ward was 5.8 days (SD 4.0) following surgery. General anaesthesia was employed in most patients, either alone (39, $38.6 \% \mathrm{n}=101)$ or in combination with neuraxial anaesthesia $(29,28.7 \%)$; the rest received neuraxial anaesthesia with sedation. 


\begin{tabular}{|c|c|c|c|c|c|c|}
\hline & \multicolumn{2}{|c|}{ HA } & \multicolumn{2}{|c|}{ KA } & \multicolumn{2}{|c|}{ Overall } \\
\hline & $\mathrm{n} /$ mean & $\% / S D$ & $\mathrm{n} /$ mean & $\% / S D$ & $\mathrm{n} /$ mean & $\% / S D$ \\
\hline $\begin{array}{l}\text { Age } \\
(n=180)\end{array}$ & 67.3 & 11.8 & 68.9 & 8.7 & 68.1 & 10.4 \\
\hline $\begin{array}{l}\text { Female gender } \\
(n=178,88 \mathrm{KA})\end{array}$ & 57 & 63.3 & 51 & 57.9 & 108 & 60.7 \\
\hline $\begin{array}{l}\text { Body mass index } \\
(n=167,85 \mathrm{KA})\end{array}$ & 30.2 & 7.2 & 32.3 & 5.3 & 31.3 & 6.3 \\
\hline $\begin{array}{l}\text { Length of stay } \\
(n=177,87 \mathrm{KA})\end{array}$ & 6.3 & 5.1 & 5.3 & 2.1 & 5.9 & 4.0 \\
\hline $\begin{array}{l}\text { Anticoagulant use prior to } \\
\text { admission }(n=180)\end{array}$ & 11 & 12.2 & 3 & 0.1 & 14 & 7.8 \\
\hline $\begin{array}{l}\text { Antiplatelet use prior to } \\
\text { admission }(n=180)\end{array}$ & 20 & 22.2 & 33 & 36.7 & 53 & 29.4 \\
\hline
\end{tabular}

\subsubsection{Contraindications to Thromboprophylaxis}

One patient was confused and persistently removing her clothes during her admission; she was deemed to be unsuitable for any prophylaxis by staff. Another patient had a history of leg ulcers and was deemed unsuitable to receive mechanical prophylaxis.

\subsubsection{Mechanical Thromboprophylaxis}

Seventy-seven patients $(43.3 \%)$ had documented mechanical prophylaxis use; all were prescribed GCS. There was no statistically significant difference in the BMI between patients with documented GCS use and those without. There was also no other significant differences in the demographics of patients prescribed mechanical prophylaxis.

\subsubsection{Pharmacological Thromboprophylaxis}

Thromboprophylaxis records were unavailable for one patient. The rest of the cohort received some form of pharmacological prophylaxis as an inpatient, which in the absence of complications, was continued throughout their admission. Anticoagulants were most commonly prescribed, particularly LMWH (Table 17). The most common antiplatelet agent prescribed was aspirin \pm dipyridamole $(90.0 \%)$. Fourteen patients $(7.9 \%)$ were prescribed an anticoagulant and an antiplatelet concurrently during their inpatient stay. 
Table 17. Inpatient and discharge thromboprophylaxis.

\begin{tabular}{|c|c|c|c|c|}
\hline \multirow[b]{2}{*}{ Agent } & \multicolumn{2}{|c|}{ Inpatient $(N=178)^{* \#}$} & \multicolumn{2}{|c|}{ Discharge $(\mathrm{N}=162)^{* \wedge^{\#}}$} \\
\hline & $n$ & $\%$ & $\mathbf{n}$ & $\%$ \\
\hline LMWH & 157 & 88.2 & 100 & 61.7 \\
\hline Antiplatelet & 10 & 5.6 & 19 & 11.7 \\
\hline Rivaroxaban & 7 & 3.9 & 4 & 2.5 \\
\hline Warfarin & 4 & 2.2 & 4 & 2.5 \\
\hline Nothing & - & - & 35 & 21.6 \\
\hline
\end{tabular}

NB: *Excludes patient with contraindication to pharmacological prophylaxis. ^Excludes patients who developed a bleed (5), wound infection (5) or VTE (6) in hospital. "Excludes 1 patient with unknown thromboprophylaxis (both during inpatient period and at discharge).

Anticoagulants were the most commonly prescribed agent at discharge, with most being newly initiated in hospital $(95,88.0 \%, \mathrm{n}=108)$. Almost two thirds of all patients prescribed an antiplatelet at discharge were identified as using one preadmission $(11,57.9 \%)$. There was no difference in thromboprophylaxis prescribing for patients discharged home and those discharged elsewhere.

\subsubsection{Hospital Comparison}

Most patients at each hospital received an anticoagulant agent during their inpatient stay (Table 18).

Table 18. Comparing hospitals' inpatient prescribing patterns $(n=178)$.

\begin{tabular}{|c|c|c|c|c|c|c|c|c|c|c|c|c|}
\hline \multirow{2}{*}{$\begin{array}{l}\text { Inpatient } \\
\text { prescription }\end{array}$} & \multicolumn{12}{|c|}{ Hospital (\%) } \\
\hline & & 1 & & 2 & & 3 & & 4 & & 5 & & 6 \\
\hline Aspirin & 5 & (16.7) & 0 & - & 0 & - & 5 & (17.2) & 0 & - & 0 & - \\
\hline Anticoagulant & 25 & (83.3) & 30 & (100.0) & 30 & (100.0) & 24 & (82.7) & 29 & (100.0) & 30 & (100.0) \\
\hline
\end{tabular}

NB: Excludes patient with contraindication to pharmacological prophylaxis (1), and patient with unknown thromboprophylaxis (1).

There was greater variation in thromboprophylaxis prescribing at discharge: aspirin use and prescribing nothing at discharge were more common at hospitals 1 and 6 respectively; and hospital 3 was the only hospital to use anticoagulants exclusively (Table 19).

Table 19. Comparing hospitals' discharge prescribing patterns $(n=162)$.

\begin{tabular}{|c|c|c|c|c|c|c|c|c|c|c|c|c|}
\hline \multirow{2}{*}{$\begin{array}{l}\text { Discharge } \\
\text { prescription }\end{array}$} & \multicolumn{12}{|c|}{ Hospital (\%) } \\
\hline & \multicolumn{2}{|r|}{1} & \multicolumn{2}{|r|}{2} & \multicolumn{2}{|r|}{3} & \multicolumn{2}{|r|}{4} & \multicolumn{2}{|r|}{5} & \multicolumn{2}{|r|}{6} \\
\hline Nothing & 9 & (32.1) & 9 & (34.6) & 0 & - & 5 & (20.0) & 1 & $(4.0)$ & 11 & (39.3) \\
\hline Aspirin & 13 & (46.5) & 1 & (3.8) & 0 & - & 5 & (20.0) & 0 & - & 0 & - \\
\hline Anticoagulant & 6 & (21.4) & 16 & (61.6) & 30 & (100.0) & 15 & (60.0) & 24 & (96.0) & 17 & (60.7) \\
\hline
\end{tabular}

NB: Excludes patient with contraindication to pharmacological prophylaxis (1), patient with unknown thromboprophylaxis (1), and any patients who developed a complication in hospital $(n=16)$. 


\subsubsection{Contemporary Guidelines}

Unfortunately datasets were not returned complete from some hospitals, particularly in relation to creatinine clearance data, thromboprophylaxis doses and prescribed thromboprophylaxis course duration data. Consequently, assessing adherence to contemporary guidelines was not possible for many patient records. This has resulted in particularly low absolute numbers in this section of the analysis.

Excluding patients who were prescribed an anticoagulant for a pre-existing medical condition and patients who developed postoperative complications, twenty-two knee patients $(68.9 \%, \mathrm{~N}=32)$ and thirteen hip patients $(39.4 \%, \mathrm{~N}=33)$ received NHMRC recommended thromboprophylaxis (Figure 18). Hip arthroplasty patients were less likely to receive the recommended course of thromboprophylaxis compared to knee arthroplasty patients $(\mathrm{p}<0.02)$. Patients who were prescribed thromboprophylaxis that was adherent with the NHMRC Guideline tended to have a higher BMI than those who did not (32.9 vs. 29.6, p = 0.03). There was no significant association between BMI and surgery type.

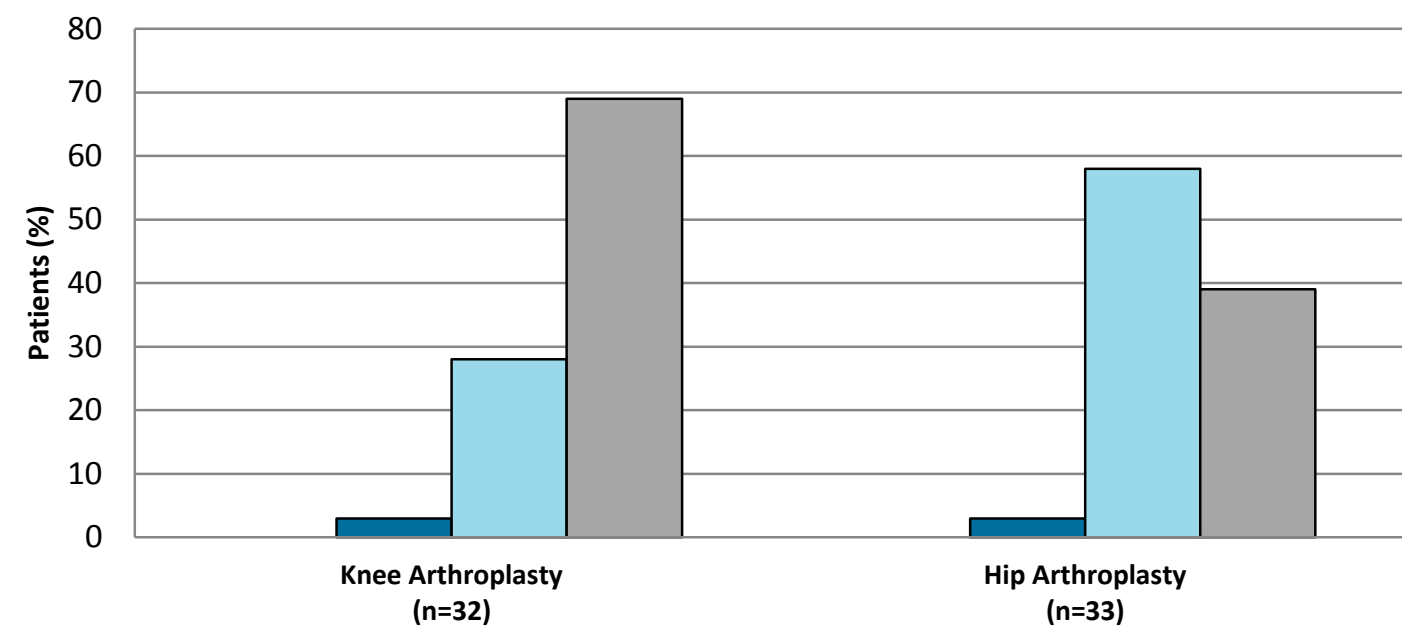

Figure 18. Proportion of patients who received NHMRC recommended prophylaxis ( $N=65)$.

Nothing appropriate

Appropriate agent and dose only
Appropriate agent only

Appropriate agent, dose and duration

Figure 19 represents a larger set of data $(n=100,61.7 \%$ of those eligible $(N=162))$ as the dosage data was removed from the analysis. Once more, hip arthroplasty patients were less likely to receive the full duration of prophylaxis compared to knee arthroplasty patients ( 21 vs. $29,39.6 \%$ vs. $61.7 \%, \mathrm{p}<0.05)$. 


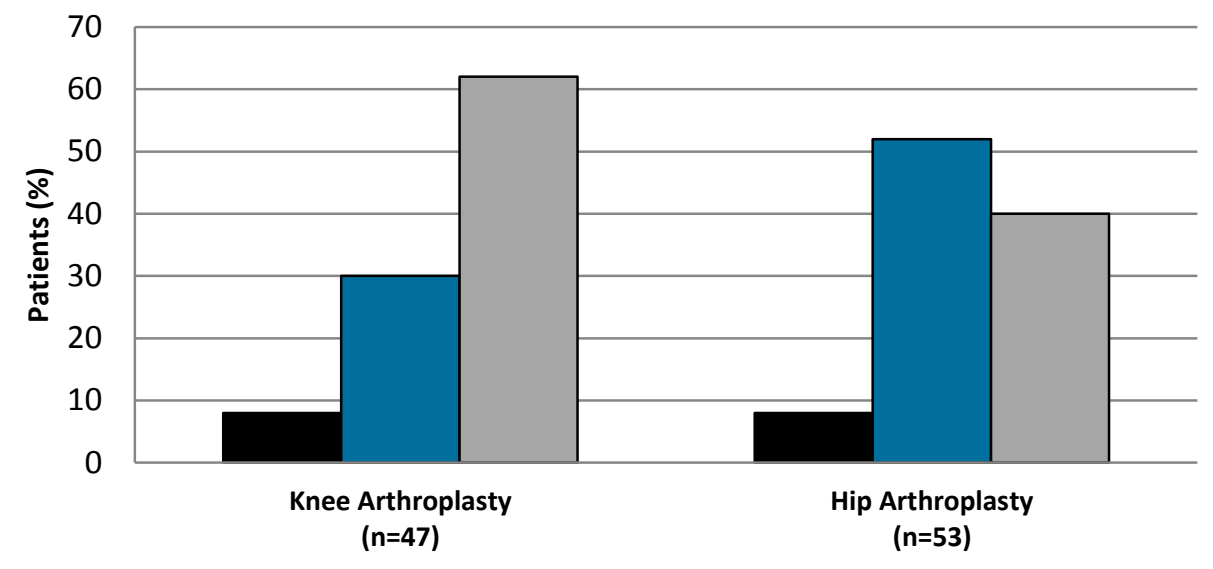

Figure 19. Proportion of patients who received NHMRC recommended prophylaxis $(N=100)$.

Nothing appropriate

Appropriate agent only

Appropriate agent and duration

\subsubsection{Postoperative Complications}

\subsubsection{Bleeding and Infections}

Five patients $(3.5 \%)$ developed a bleeding complication, and five patients (3.5\%) developed a wound or joint infection postoperatively $(n=141)$. Interestingly, men were more likely to develop bleeding complications postoperatively than women $(7.1 \%$ vs. $0.9 \%, \mathrm{p}<0.05)$, and all infective complications were reported in knee arthroplasty patients. There were no other significant associations between bleeding and infection risk and patient demographics.

\subsubsection{Symptomatic VTE}

Six patients $(4.3 \%, \mathrm{n}=141)$ developed VTE in hospital ( 2 hip patients, 4 knee patients), with no significant difference in the characteristics of those who did and those who didn't develop VTE. Five patients developed a DVT and one patient developed a PE. All patients who developed VTE postoperatively received an anticoagulant at some stage during their inpatient stay. Four of the patients who developed a DVT were being given enoxaparin 40mg daily at the time of their DVT diagnosis; two of these patients had a history of hypertension, and heart disease; one patient had dyslipidaemia; and the other only had a past medical history of osteoarthritis and associated pain.

The other two patients who developed VTE (one PE, one DVT) were on either an inappropriate agent or dose of thromboprophylaxis at the time their VTE developed. The patient who developed a DVT had initially received rivaroxaban 10mg daily for three days. He was then given aspirin 100mg and developed a DVT twenty four days later on the rehab ward. The patient who developed a PE had a history of type 2 diabetes and peripheral vascular disease, and despite a healthy admission creatinine clearance $(67 \mathrm{~mL} / \mathrm{min})$ initially received enoxaparin $20 \mathrm{mg}$ postoperatively instead of the recommended 40mg. Following her PE she was prescribed 
enoxaparin $80 \mathrm{mg}$ twice daily for the rest of her inpatient stay (4 days); and at discharge she received six weeks of low dose aspirin.

Unfortunately it was not possible to determine the median number of days to postoperative complication (VTE, bleeding or infection) in this study.

\subsection{Discussion}

This review identified that inpatient thromboprophylaxis prescribing was similar across the surveyed public hospitals, with many patients receiving anticoagulant thromboprophylaxis as recommended by the NHMRC Guideline. Prescribing at discharge however varied between hospitals, with one third $(54,33.3 \%)$ of patients receiving either an antiplatelet agent, or nothing at discharge. Overall, a little over half of the patients with full datasets were prescribed an NHMRC recommended agent, dose and thromboprophylaxis course $(35,53.8 \%, \mathrm{n}=65)$.

Although it was not an aim of this study, the review identified a significant complication risk associated with the surgeries, with 16 patients $(11.3 \%)$ developing a postoperative complication while in hospital: five (3.5\%) developed a bleed, five (3.5\%) developed a wound or joint infection, and six patients developed a VTE $(4.3 \%, \mathrm{~N}=141)$.

Inpatient prescribing in this review was similar to that outlined in Chapter 3; most patients were prescribed anticoagulants following surgery, in particular LMWH. Furthermore, when RHH data was separated out from the rest and compared to the Chapter 3 data there was no significant difference in pharmacological prophylaxis prescribing. This suggests that RHH prescribing practices had not significantly changed in the 18 months between the two studies. Noteworthy however, was the drop in documented mechanical prophylaxis use (from $14.0 \%$ to $0 \%$ ).

Even though inpatient prescribing in this multicentre study was not significantly different to the RHH data in Chapter 3, there was a significant difference in the prescribing of thromboprophylaxis at discharge. In particular, significantly more patients were prescribed an anticoagulant at discharge across the multiple centres (66.7\% vs. 16.3\%); and a significantly greater proportion of patients went home with some form of pharmacological thromboprophylaxis (78.4\% vs. $36.7 \%)$. Interestingly antiplatelets were less commonly prescribed at discharge in this cohort, and most patients prescribed an antiplatelet agent were using one prior to admission.

The proportion of patients who developed VTE postoperatively in hospital was significantly higher in this study than the previous study $(4.3 \%$ vs. $0.7 \%)$. As previously noted, VTE occurs

more commonly after discharge. ${ }^{51,62,143,184,185}$ It is likely then that the 90 -day incidence would have been close to double the previous study (2.7\%), however only a 90 day follow up with the cohort could confirm this. 
A major limitation of this study is the significant number of patient records returned with missing data. This is because some data (e.g. discharge prescribing data and lab data) were not readily available in the paper based hospital files that were reviewed by investigators. This lack of data ultimately limited the adherence analysis in particular. Nevertheless, similar to the previous study, this study identified that patients with increased BMI tended to be more likely to receive guideline approved thromboprophylaxis. Remarkably, a significantly greater number of patients received 'appropriate' thromboprophylaxis in this study, compared to the study outlined in Chapter $3(53.8 \%$ vs. $5.1 \%)$.

Remarkably two hospitals' orthopaedic departments vetoed this study because of its auditing nature, increasing nonresponse bias risk. It is possible that these hospitals may have been less likely to adhere with the NHMRC Guideline recommendations than those included in the study; however, only a follow up with these hospitals would be able to confirm this. Whilst it is unfortunate that only six hospitals were able to participate, the data confirmed that the guidelinepractice gap identified in Chapter 3 was not unique to the RHH. 


\section{Chapter Five: Australian Hospital Protocols}

\subsection{Introduction}

The studies outlined in Chapters 3 and 4 confirmed that despite the presence of national and international guidelines, thromboprophylaxis prescribing is variable in both Tasmania and interstate. Anecdotal evidence suggested some hospitals had their own thromboprophylaxis protocols that differed from the contemporary guidelines. A national survey of hospitals was conducted to identify if these protocols had any impact on prescribing.

\subsection{Aim}

The aims of this survey were four-fold:

- to determine how many Australian hospitals have thromboprophylaxis protocols,

- where protocols exist, to identify what they are based on and how effectively they are being used in practice,

- where protocols exist but are not being extensively used, to identify potential barriers to their uptake, and

- to identify strategies being effectively employed to improve adherence to protocols.

\subsection{Methods}

\subsubsection{Participant Selection and Invitation}

Potential participants were identified by reviewing the list of hospitals that contribute data to the National Joint Replacement Registry (http://aoanjrr.dmac.adelaide.edu.au), and through reviewing the list of hospitals with orthopaedic services on the Australian government's MyHospitals website (http://www.myhospitals.gov.au). A list of 363 hospitals was compiled using these means. These hospitals were then contacted via telephone to determine if they offered hip and/or knee arthroplasty services at the hospital (as opposed to other orthopaedic services). Finally, the YellowPages and MyHospitals websites were used to develop an address list for 310 hospitals (158 private, 152 public).

A letter was sent to the orthopaedic clinical nurse manager (CNM) at each hospital inviting them to participate on behalf of their hospital (Appendix B). As a strategy to encourage participation, each CNM who completed the survey was offered the opportunity to enter into a draw for a AUD250 gift voucher. 
Despite the gift voucher incentive, the initial response rate was only $\sim 10 \%$. A review of the literature identified that other studies have found that sending an advance postal survey notice, using unconditional monetary incentives (e.g. gift certificates), employing handwritten notes, and/or sending large numbers of reminders can improve survey response rates. ${ }^{191-194}$ After reviewing all of these strategies and more, three changes were implemented to improve the response rate of this survey. Firstly, an ethics amendment was submitted and approved to increase the participation incentive to a first place prize of a third generation Apple iPad (valued at AUD539), with the AUD250 gift voucher subsequently becoming a second prize. Secondly, as some CNMs had difficulty accessing the survey online, a hard copy of the survey was included in each reminder letter along with a reply-paid envelope. Thirdly, each and every letter was personally signed by the researcher, and included a little post-it thank you note.

\subsubsection{The Survey}

The survey was initially created online with the LimeSurvey open source PHP web application software (http://limesurvey.org/) and hosted on a University of Tasmania server (Appendix B). It was divided into three sections: hospital details; thromboprophylaxis protocols and their evidence base; and barriers to the uptake of protocols (modified from data presented in Figure 10). The survey concluded with space for participants to share any further comments on thromboprophylaxis.

The survey was available online for three months from March 2012 to June 2012. During this time two reminder letters (each with a hard copy of the survey enclosed) were sent. Notably, once the option to complete the survey on paper was implemented all of the responses received thereafter were in the hard copy format.

\subsubsection{Statistics}

All information was stored in an Access database (Microsoft ${ }^{\circledR}$ Access 2010), and analysed using SPSS 19.0. The differences between groups were tested using the t-test for independence for continuous data, and the chi square test for categorical variables. Continuous variables were summarised as means and standard deviation. A univariate analysis, followed by a multivariate analysis of variables, was performed to assess the contribution of individual hospital variables on the likelihood of a hospital having a formal protocol. Only $\mathrm{p} \leq 0.05$ values were considered statistically significant. 


\subsection{Results}

One hundred and forty-three surveys $(46.1 \%)$ were returned in total; however, thirteen respondents reported that their hospital did not offer hip or knee arthroplasties and a further four surveys were returned mostly empty. This resulted in a final response rate of $42.8 \%$ (126 surveys, N=294).

Although this survey was addressed to clinical nurse managers, a range of other health professionals responded, including: hospital CEOs, theatre staff, heads of orthopaedic departments (HODs), clinical pharmacists, registered nurses, registrars, residents, interns, physiotherapists, hospital and nurse educators, haematologists, anaesthetists, surgical ward managers, nurse practitioners, coordinators of clinical policy and clinic managers.

\subsubsection{Demographics}

Hospitals responded from every state and territory in Australia; unfortunately the only one returned from the Northern Territory was not viable for analysis. The response rates from Victoria, New South Wales, and Queensland based hospitals were only 38.4\%, 36.4\% and 35.0\% respectively; these states provided the bulk (70.6\%) of completed responses (Figure 20). The best response rates were from the Australian Capital Territory (100\%), Western Australia (75.0\%) and Tasmania (66.7\%). Two participants did not identify which state their hospital was in.

Figure 20. Response rate from each state.

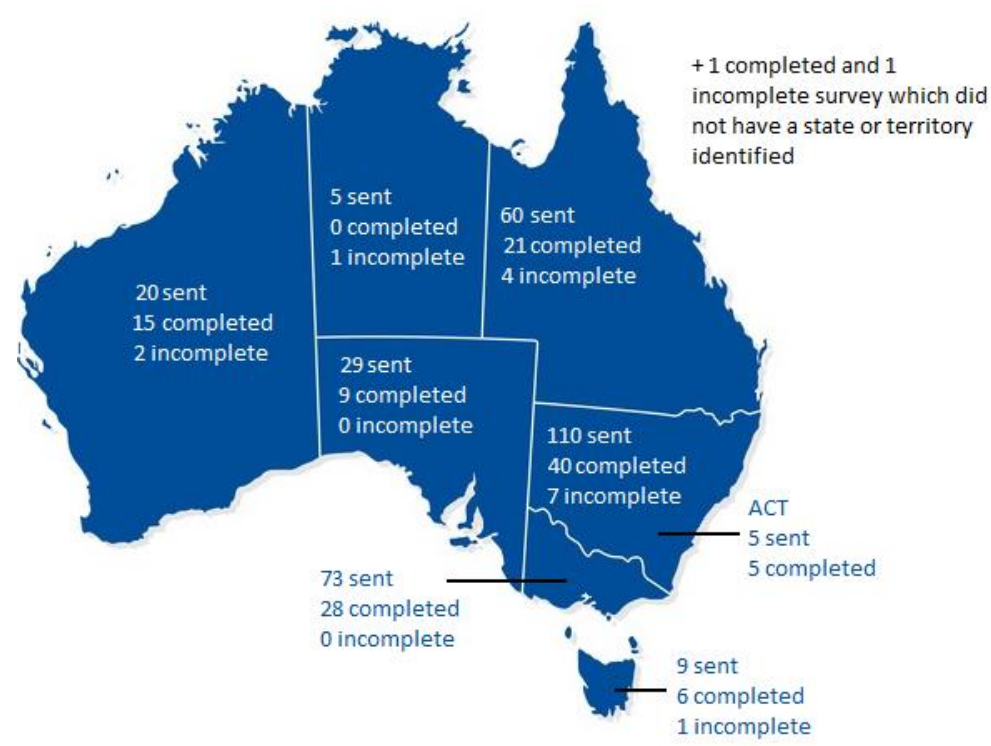

Seventy-one (56.3\%) responding hospitals were private hospitals, two were unmarked and the remaining fifty-three $(42.1 \%)$ were public hospitals. The hospitals had a combined total of 20,575 beds, averaging 171 beds per hospital (SD 159, n=120, Table 20). Public hospitals had more beds on average than private hospitals ( 216 vs. 138 respectively, $\mathrm{p}=0.01$ ). 
Table 20. Hospital demographics.

\begin{tabular}{|l|l|l|l|l|l|l|l|l|l|}
\hline & \multicolumn{3}{c|}{ Public Hospitals } & \multicolumn{3}{c|}{ Private Hospitals } & \multicolumn{3}{c|}{ Overall } \\
\hline & $\mathbf{n}$ & mean & SD & $\mathbf{n}$ & mean & SD & mean & SD \\
\hline Beds* & 52 & 215.6 & 187.5 & 68 & 137.6 & 123.8 & 171 & 158.8 \\
\hline Surgeons & 55 & 5.6 & 4.2 & 71 & 5.9 & 5.6 & 5.8 & 5.0 \\
\hline Hip arthroplasties* & 53 & 103.2 & 98.8 & 67 & 181.7 & 210.1 & 147.0 & 174.0 \\
\hline Knee arthroplasties* & 52 & 112.7 & 121.9 & 67 & 217.3 & 190.0 & 171.6 & 171.3 \\
\hline Total arthroplasties* & 53 & 213.7 & 214.3 & 67 & 399.0 & 386.9 & 317.2 & 334.1 \\
\hline
\end{tabular}

NB: * indicates statistically significant difference between hospital sector.

A combined total of 715 orthopaedic surgeons were reported to operate at the responding hospitals. This averaged to 6 surgeons per hospital regardless of sector (SD 5, n=126); however, it is likely there was some overlap as surgeons often operate at more than one hospital.

The annual number of arthroplasties conducted at each hospital varied considerably from 4 to 1600 surgeries. Public hospitals reported significantly lower arthroplasty numbers than private hospitals ( 214 vs. 399 respectively, $\mathrm{p}=0.001$ ). Reflective of the national trend, both sectors reported a trend towards conducting more knee arthroplasties than hip arthroplasties (172 knees vs. 147 hips annually); however, this did not reach statistical significance.

\subsubsection{Thromboprophylaxis Protocols}

Sixty three respondents $(50.0 \%)$ reported having a formal thromboprophylaxis protocol at their hospital (26 private hospitals, 37 public hospitals). Multivariate analyses identified that the total number of arthroplasties conducted at a hospital was the only factor that had any statistically significant impact on whether a hospital reported having a formal thromboprophylaxis protocol or not. Hospitals that reported having a formal protocol in place conducted less arthroplasties (262 vs. $374, \mathrm{p}=0.07$ ), were more likely to have more beds (202 vs. $140, \mathrm{p}<0.05$ ), and were almost twice more likely to be a public hospital than a private hospital $(67.2 \%$ vs. $36.6 \%, \mathrm{p}<0.001)$.

\subsubsection{Evidence Base}

Only eight respondents attached their thromboprophylaxis protocol to the survey $(12.7 \%)$; however, most participants indicated the evidence base for their protocol. The NHMRC Guideline was the most common basis on which thromboprophylaxis protocols were reportedly developed or based on (Figure 21). 


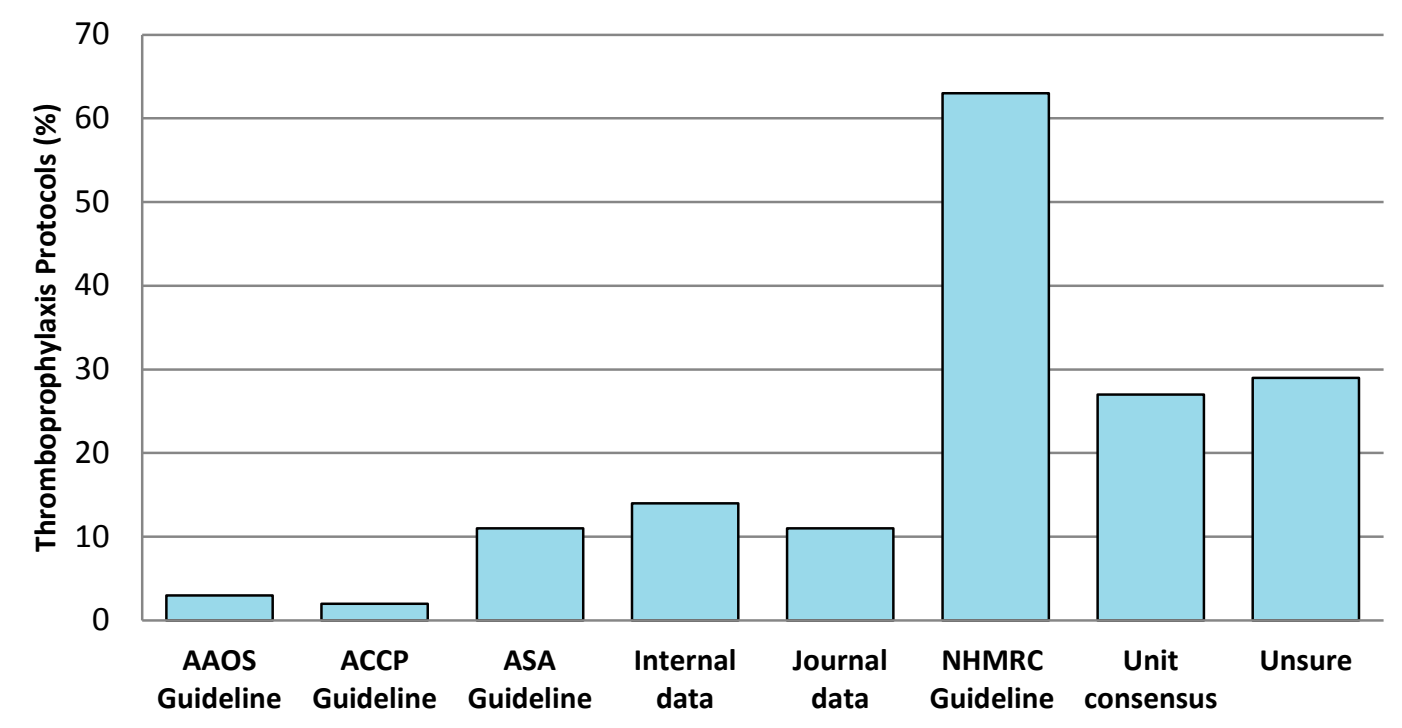

Figure 21. Basis of thromboprophylaxis protocols in responding hospitals $(\mathrm{N}=63)$.

NB: Some respondents indicated multiple sources for their hospital protocol

Private hospital protocols were more likely to be based on the NHMRC Guideline $(81.5 \%$ vs. $51.3 \%, \mathrm{p}<0.05$ ), and public protocols were more likely to be based on unit consensus (40.5\% vs. $7.4 \%, \mathrm{p}<0.05)$.

\subsubsection{Protocol Adherence}

Most protocols were described as mandatory $(45,72.5 \%, \mathrm{n}=62)$, with staff reportedly familiar with protocols in $95.1 \%$ of hospitals. Protocol adherence was reported to be high (86.3\% adherence, SD 19.7) and was not impacted by protocol evidence base, hospital state/territory, or hospital sector. HODs were more likely to report high adherence to protocols than any other health professionals $(\mathrm{n}=6,95.4 \%$ vs. $85.4 \%, \mathrm{p}<0.01)$.

\subsubsection{Adherence Improvement Strategies}

Approximately two thirds of hospitals with protocols reported having policies in place to encourage adherence (40,63.5\%). Most commonly, these policies included embedding prophylaxis into clinical pathways (preadmission, inpatient and at discharge), regular audits and staff education (Table 21). 
Table 21. Reported hospital policies to encourage protocol adherence $(\mathrm{N}=63)$.

\begin{tabular}{|l|l|l|}
\hline Theme & $\mathbf{n}$ & $\mathbf{\%}$ \\
\hline Regular prescribing audits conducted & 15 & 24 \\
\hline Embedded in inpatient processes e.g. clinical pathway, prompts, handover checks & 14 & 22 \\
\hline Staff education (new and continuing staff) & 11 & 17 \\
\hline Embedded in discharge process & 10 & 16 \\
\hline Embedded in preadmission process e.g. preadmission written education & 9 & 14 \\
\hline Thromboprophylaxis part of drug chart & 6 & 10 \\
\hline Hospital expectation all staff follow policy & 8 \\
\hline Risk assessment tools e.g. stickers in inpatient notes & 5 & 6 \\
\hline Protocol placed in visual spot & 4 & 5 \\
\hline VTE committee or expert involvement & 3 & 3 \\
\hline
\end{tabular}

There was a trend towards increased adherence in hospitals with adherence strategies, particularly following hip arthroplasty (91.0\% vs. $79.2 \%, \mathrm{p}=0.08)$. This was most markedly evident in hospitals that had embedded thromboprophylaxis into regular practice and implemented staff education strategies.

\subsubsection{Barriers to Protocol Uptake}

One hundred and eighteen participants $(93.6 \%)$ completed this component of the survey $(50.8 \%$ from hospitals with protocols). However, many of them $(43,36.4 \%)$ did not consider any of the listed perceptions as barriers to the uptake of protocols. Of those that answered otherwise, the perception that protocols interfere with professional autonomy was the most commonly perceived barrier to protocol update (Figure 22). 


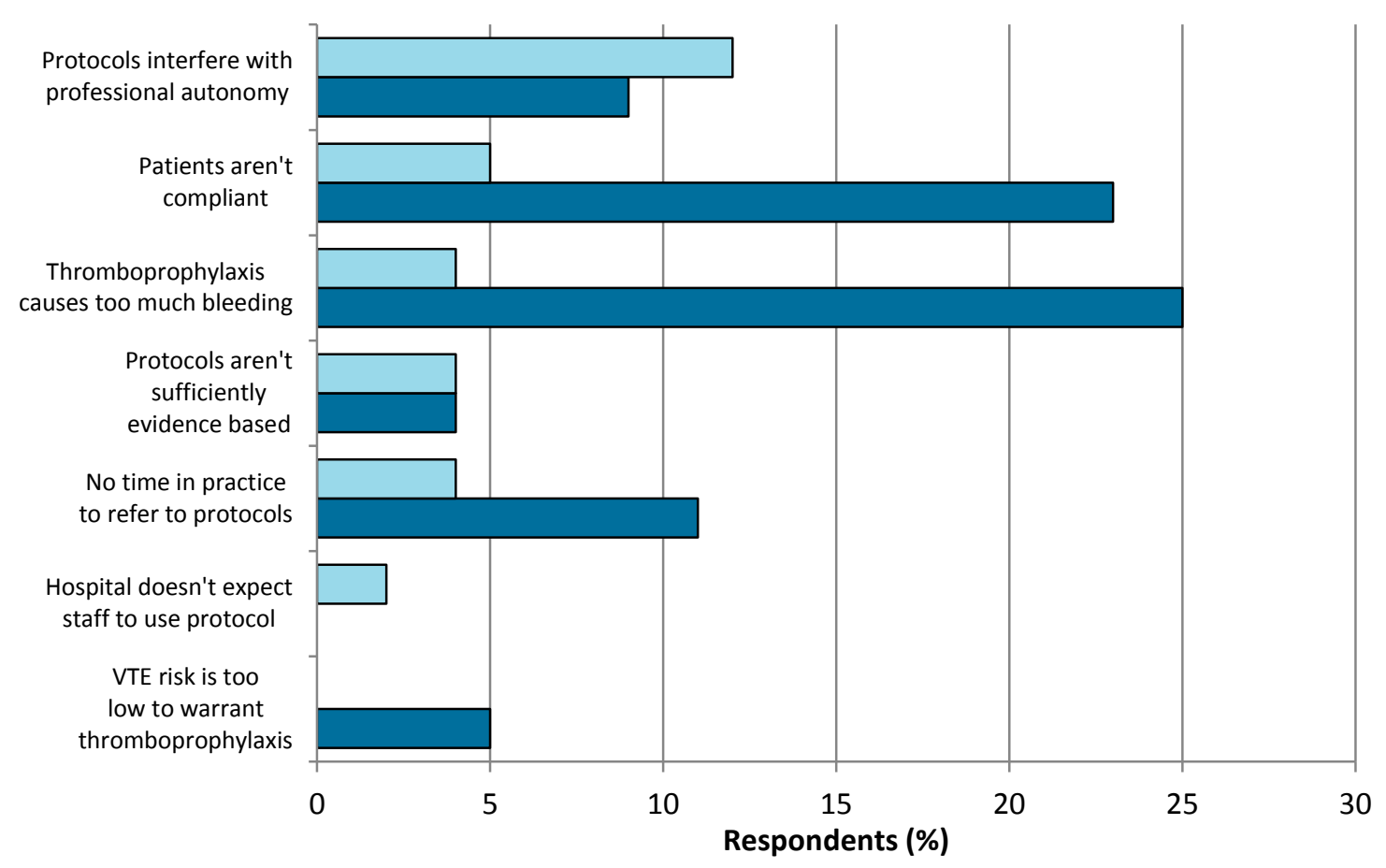

Figure 22. Potential barriers to protocol uptake $(\mathrm{N}=118)$.

$\square$ Yes $\square$ Maybe

\subsubsection{Hospitals without Protocols}

Of the 63 hospitals with no reported protocol, 60 (95.2\%) indicated that their orthopaedic surgeons had their own protocol. Respondents were less likely to know what these protocols were based on compared to formal protocols ( $24.6 \%$ vs. $6.6 \%$, p <0.01) (Figure 23). Surgeons were more likely to have their own protocol in private hospitals than public hospitals $(100 \%$ vs. $83.3 \%$, $\mathrm{p}<0.05)$.

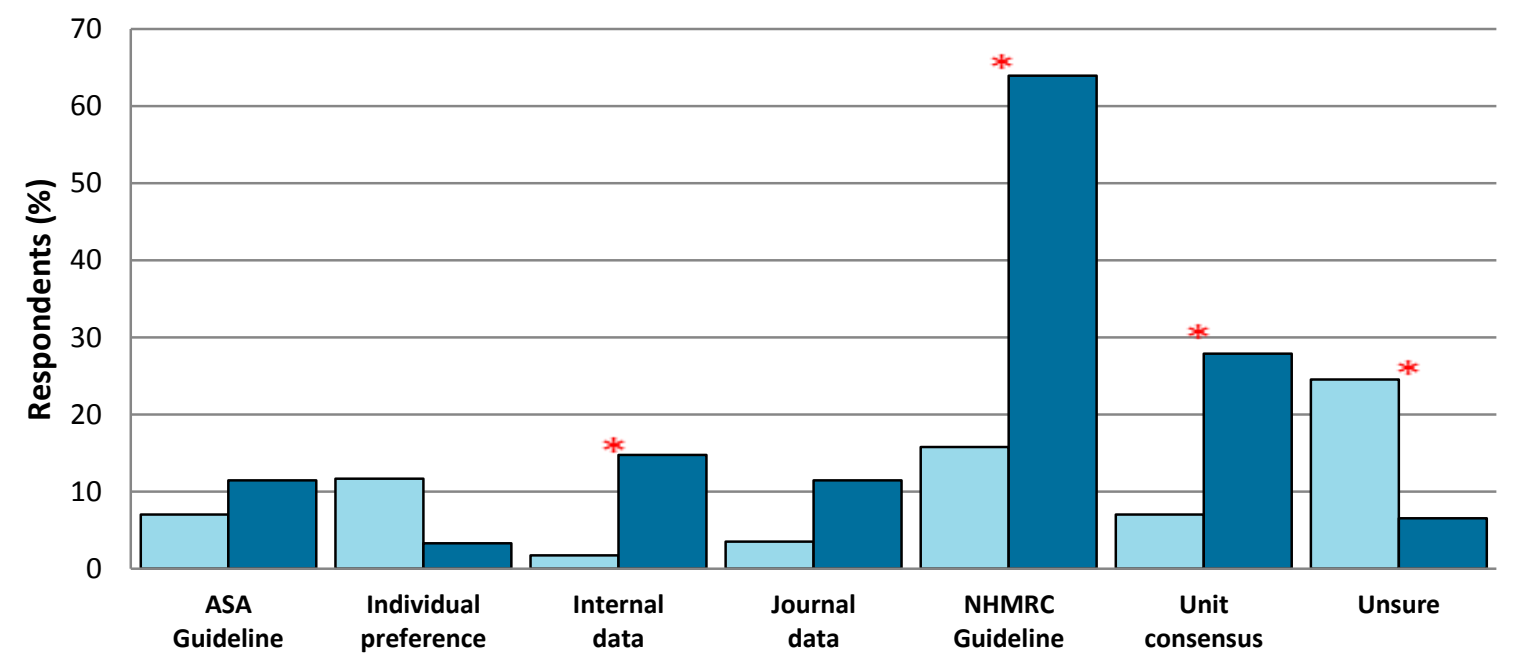

Figure 23. Basis of thromboprophylaxis protocols in responding hospitals $(N=123)$.

$\square$ No formal protocol $\square$ Formal protocol in place. ${ }^{*}$ indicates statistically significant difference $(p<0.05)$. 


\subsection{Discussion}

This study identified that half of the hospitals surveyed had a formal thromboprophylaxis protocol in place. Interestingly, these hospitals tended to have more beds, but were reported to conduct fewer hips and knee arthroplasties each year. Where protocols existed, they were more likely to be based on the NHMRC Guideline than any other evidence base. Adherence to protocols was relatively good, with over $85 \%$ of arthroplasty patients reportedly receiving thromboprophylaxis according to their hospital or unit protocol, irrespective of the basis of the protocol. Implementation of adherence strategies have been previously reported to improve VTE risk assessments and thromboprophylaxis use; and similarly, there was a trend towards hospitals with adherence strategies in place in this study having higher adherence rates, however this did not reach statistical significance. ${ }^{177,195}$

Relatively few participants identified potential barriers to the uptake of protocols in their hospitals, potentially due to their use of thromboprophylaxis already being so high. The biggest perceived barrier to protocol uptake was the perception that protocols interfere with surgeon autonomy. This may be because clinical practice guidelines, and similarly protocols, can be considered a threat to clinical and professional autonomy if they are seen as too prescriptive specifying how health care should be performed. ${ }^{175,196}$ Similar to the findings reported in 2.4.1.2 Barriers, bleeding concern was also identified in this survey as a potential (though not significant) barrier to pharmacological prophylaxis use. ${ }^{182,197}$ Patient compliance and a lack of time to refer to protocols in practice were also identified as potential barriers to protocol uptake.

Unexpectedly, public hospitals were more likely to report protocols based on unit consensus, and less likely to report protocols based on the NHMRC Guideline.Almost all of the hospitals without a hospital or unit protocol in place indicated that their orthopaedic surgeons had their own protocol. This suggests orthopaedic surgeons are inclined to carefully consider the postoperative thromboprophylaxis needs of their patients. That some surgeons base their individual protocol on contemporary guidelines suggests they are reviewing these guidelines as individuals, even if their hospital or unit are not. Although this survey was addressed to clinical nurse managers, a range of other health professionals responded. Interestingly, HODs were more likely to report high adherence to protocols than any other health professionals. This may be due to the proactive nature of these HODs (as exemplified in them taking the time to fill out a survey addressed to CNMs), or due to a desire to promote their hospital as adherent to guidelines

Surprisingly, the response rate from private hospitals was better than from public hospitals (44.3\% vs. $34.9 \%)$. An interesting aspect of this study was the large impact a few tweaks to its method made on the response rate. Providing a means to complete and return a hard copy of the survey, increasing the prize draw, and adding a personal touch increased the response rate by over $450 \%$. It is likely all of these elements assisted together as $75.4 \%$ of respondents chose to complete 
the hard copy of the survey and many sent personal 'good luck' and 'thank you' notes back with their responses (as shown below). Furthermore, some participants emailed the researcher separately to the mail back to ensure that they were in the draw for the iPad.

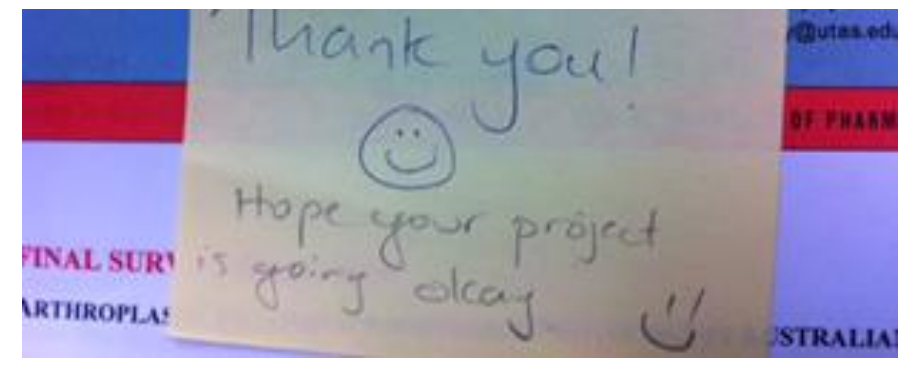




\section{Chapter Six: Survey of Australian ORThOPAEdic Surgeons}

\subsection{Introduction}

The retrospective audits in Chapters 3 and 4 identified that many arthroplasty patients did not receive recommended thromboprophylaxis courses in Australia, in 2010 and 2011. The results of Chapter 5 (conducted in 2012) shed a little light on factors that influence thromboprophylaxis use and protocol uptake in hospitals. To further complement the retrospective data as it was collected, and to further explore factors that influence prescribing, a number of projects centered on surveying and interviewing surgeons were conducted. The first of these was an online survey of orthopaedic surgeons that was conducted in late 2010.

\subsection{Aim}

The aim of this survey was two-fold:

- to identify what thromboprophylaxis trends exist in Australia for arthroplasty patients;

- and to identify enablers and barriers to thromboprophylaxis prescribing, from the perspective of orthopaedic surgeons.

\subsection{Methods}

\subsubsection{Participant Selection and Invitation}

An invitation to participate in the survey was distributed by the Arthroplasty Society of Australia (ASA) to its 103 members (Appendix C). The AOA was approached initially to seek assistance in recruiting orthopaedic surgeons. However, it replied that 'many surgeons perform few or no joint replacements in the lower limb' and anticipated the response rate would be poor. The Association suggested collaborating with the ASA whose members specialise in joint replacements (at least $80 \%$ of the surgeries they conduct must be joint replacements). Fortunately, the ASA agreed to distribute electronic invitations with a link to the survey online.

\subsubsection{The Survey}

An electronic survey was created with the LimeSurvey open source PHP web application software (http://limesurvey.org/), and hosted online on a University of Tasmania server (Appendix C). It was divided into five sections covering: demographic and professional practising information; perceived thromboprophylaxis effectiveness, preferred agents and duration of use; factors that 
influence prophylaxis prescribing during the inpatient and discharge period; four case studies designed to assist in the identification of factors that influence thromboprophylaxis prescribing; and questions concerning institution-specific guidelines and contemporary guidelines. The case studies were designed to assess the impact of age, weight, medications and past history of bleeding and VTE on thromboprophylaxis prescribing.

The survey concluded with space for participants to share any further comments on thromboprophylaxis, and an invitation to participate in short interviews on thromboprophylaxis use postoperatively.

The survey was open for participants to access for 6 weeks, during which the ASA sent two electronic reminders encouraging members to participate. Furthermore, a second generation Apple iPad (valued at AUD629) prize draw was used as a strategy to improve participation.

\subsubsection{Statistics}

Data was collected using LimeSurvey and formatted for analysis with SPSS 19.0. Continuous variables were summarised as medians and ranges. The differences between groups were tested using the Mann-Whitney test for continuous data, and the chi squared test for categorical variables. Spearman's rank correlation coefficients were calculated for measuring correlations. Only $p \leq 0.05$ values were considered statistically significant.

\subsection{Results}

In total, twenty-five surgeons completed the survey (response rate: 24\%). Another surgeon started the survey but did not complete it.

\subsubsection{Demographics}

Surgeons practising in Victoria (10,40.0\%), New South Wales (7, 28.0\%), South Australia (4, $16.0 \%)$, Queensland $(3,12.0 \%)$ and Western Australia (1, 4.0\%) completed the survey. All participants were male, with a median of 20 years practice as an orthopaedic surgeon (range: 8 to 27 years). Most surgeons practised predominantly in the private sector $(23,92.0 \%)$, and conducted an average of nine hip (range: 1 to 30, total: 269) and ten knee arthroplasties (range: 1 to 30, total: 271) monthly.

\subsubsection{Prescribing Practices}

All surgeons prescribed pharmacological prophylaxis following arthroplasty, choosing LMWH $(12,48.0 \%)$, aspirin $(11,44.0 \%)$, fondaparinux $(1,4.0 \%)$ and rivaroxaban $(1,4.0 \%)$ as their agents 
of choice. There was no significant correlation between preferred agent and professional experience (years or surgeries).

Except for one participant, all surgeons routinely prescribed pharmacological prophylaxis for the entire inpatient period (Table 22). There was no correlation between the number of years practising and duration preference.

Table 22. Preferred duration of thromboprophylaxis $(\mathrm{N}=25)$.

\begin{tabular}{|l|l|l|}
\hline & Hip Arthroplasty (\%) & Knee Arthroplasty (\%) \\
\hline During inpatient stay & 4.0 & 4.0 \\
\hline At discharge & 24.0 & 24.0 \\
\hline At 14 days postoperatively & 24.0 & 36.0 \\
\hline 4 to 5 weeks postoperatively & 48.0 & 36.0 \\
\hline
\end{tabular}

\subsubsection{Thromboprophylaxis Efficacy}

When asked if pharmacological prophylaxis is effective in preventing fatal PE, $24.0 \%$ of surgeons did not believe so, $64.0 \%$ of surgeons thought it may potentially reduce fatal PE, and only three surgeons $(12.0 \%)$ believed pharmacological agents definitely decreased fatal PE following surgery.

\subsubsection{Influencing Factors}

A patient history of VTE, hormone replacement therapy (HRT) use and prolonged preoperative immobility were the top three patient factors surgeons indicated would prompt them to prescribe pharmacological prophylaxis during the inpatient and discharge period (Table 23). High bleeding risk, a perception that extended thromboprophylaxis is inconvenient, belief that pharmacological methods are not superior to mechanical methods; and a low perceived VTE risk were the top four factors felt to generally limit prophylaxis prescribing postoperatively (Table 24). 
Table 23. Patient factors that prompt pharmacological prophylaxis use $(\mathrm{N}=25)$.

\begin{tabular}{|l|l|l|l|}
\hline & Inpatient \% & Discharge \% & Overall \% \\
\hline Age $<$ 70 years & 12.0 & 4.0 & 16.0 \\
\hline Age $\geq 70$ years & 20.0 & 4.0 & 20.0 \\
\hline Current smoker & 24.0 & 8.0 & 28.0 \\
\hline General anaesthesia & 12.0 & 0.0 & 12.0 \\
\hline HRT & 44.0 & 32.0 & 52.0 \\
\hline Moderate obesity (BMI >30) & 32.0 & 12.0 & 32.0 \\
\hline Morbid obesity (BMI >40) & 28.0 & 16.0 & 36.0 \\
\hline None of these factors & 4.0 & 4.0 & 8.0 \\
\hline Preoperative infection & 12.0 & 0.0 & 12.0 \\
\hline Previous thrombophlebitis & 24.0 & 16.0 & 28.0 \\
\hline Previous vein surgery & 16.0 & 4.0 & 20.0 \\
\hline Previous VTE & 92.0 & 76.0 & 96.0 \\
\hline Prolonged preoperative immobility & 44.0 & 4.0 & 44.0 \\
\hline Surgery>2 hrs & 24.0 & 4.0 & 24.0 \\
\hline PB: BMII & & & \\
\hline
\end{tabular}

$\mathrm{NB}: \mathrm{BMI}=$ body mass index, $\mathrm{HRT}=$ hormone replacement therapy.

Table 24. Perceptions that limit pharmacological prophylaxis use $(N=25)$.

\begin{tabular}{|l|l|l|}
\hline & Inpatient \% & Discharge \% \\
\hline It increases the risk of infection at the operation site & 28.0 & 4.0 \\
\hline It is not safe as it causes too much bleeding & 68.0 & 36.0 \\
\hline It is not superior to mechanical methods & 40.0 & 8.0 \\
\hline It is too expensive & 4.0 & 12.0 \\
\hline It is too inconvenient & 48.0 \\
\hline Patients will not be compliant & 0.0 & 12.0 \\
\hline Poor continuation of care from hospital to community & 0.0 & 16.0 \\
\hline Study evidence isn't applicable to real-world populations & 4.0 & 12.0 \\
\hline The risks of VTE are low & 32.0 & 28.0 \\
\hline None of the above & 24.0 & 40.0 \\
\hline
\end{tabular}

\subsubsection{Familiarity with Contemporary Guidelines}

All surgeons indicated they were very familiar with the ASA Guideline, followed by the AAOS and NHMRC guidelines (Figure 24). Surgeons who were familiar with the NICE Guideline reported conducting less arthroplasties a month than those who had only come across it in passing (17 vs. $29, \mathrm{p}=0.01)$. There was no other significant difference in demographics. 


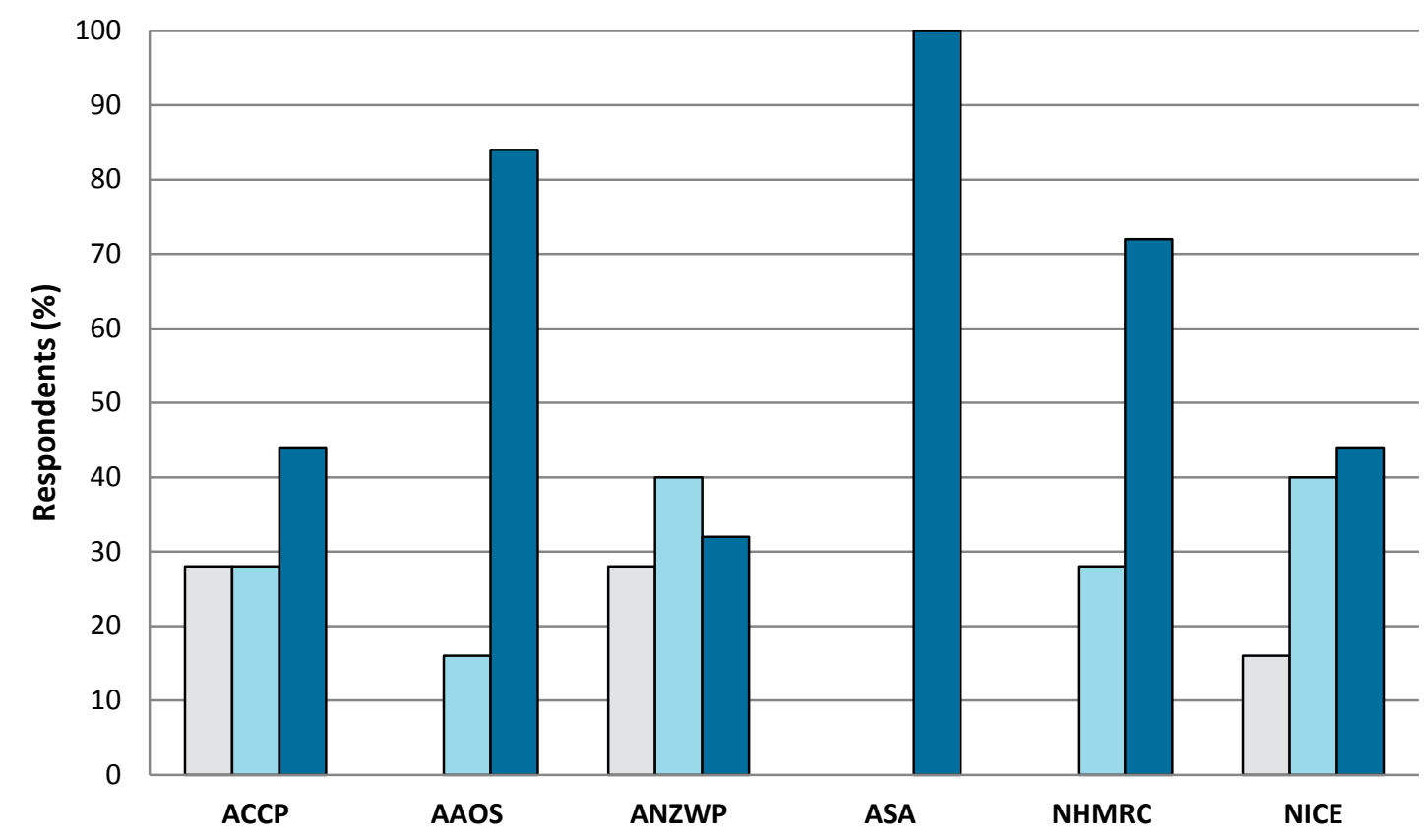

Figure 24. Surgeon familiarity with contemporary guidelines $(\mathrm{N}=25)$.

$\square$ I have not come across it before $\square$ । have heard of it in passing $\square$ I am very familiar with it

\subsubsection{Case Studies and Thromboprophylaxis Prescribing}

The first case was designed as an older lady with no history of VTE or bleeds. Participants tended to prescribe the agent they had previously stated was their preferred agent; however those surgeons who prescribed an anticoagulant tended to prescribe a lower dose than would be recommended for the patient's kidney function.

The second case was an average aged male with no history of VTE or bleeds, but was obese (BMI=31), had mild kidney impairment and was diabetic. Once again, participants prescribed their previously stated preferred agent, however despite his kidney impairment they were more inclined to choose normal doses of anticoagulants (higher than would be recommended for this patient).

The third and fourth cases were a middle aged female and a slightly older male with a significant and potentially life-threatening history of VTE and bleeding respectively. Participants deviated from their preferred agents in these cases to balance the risks associated with the cases: almost all participants $(96.0 \%)$ prescribed an anticoagulant for the patient taking hormone replacement therapy with a history of VTE; and most participants either prescribed either mechanical prophylaxis \pm aspirin for the patient with a history of haemorrhagic stroke.

\subsubsection{Case Studies and Guideline Adherence}

The four case studies were also used to compare adherence to the ASA and NHMRC guidelines. Across the cases, $68.0 \%$ and $88.0 \%$ of surgeons prescribed mechanical prophylaxis in line with ASA and NHMRC recommendations, respectively. Adherence to pharmacological prophylaxis 
recommendations was considerably lower across the cases; between $56.0 \%$ and $60.0 \%$ of participants prescribed in line with the NHMRC Guideline across the cases. This dropped to between $16.0 \%$ and $56.0 \%$ across the cases when doses were reviewed (as per product information). Between $56.0 \%$ and $96.0 \%$ of participants prescribed an ASA recommended agent, and between $44.0 \%$ and $84.0 \%$ prescribed it at an appropriate dose.

As there are no 'duration of therapy' recommendations in the ASA Guideline, and the NHMRC recommendations are vague (up to 14 days for knees, up to 35 days for hips) duration of therapy was not compared to guideline recommendations. In general, however, there was a trend towards surgeons prescribing longer anticoagulant courses for the case with a previous history of VTE (80.0\% chose to prescribe an anticoagulant for 4 weeks or longer), and no anticoagulant agent for the case with a previous history of haemorrhagic stroke (56.0\% chose not to prescribe anything, and $32.0 \%$ chose to prescribe aspirin solely).

\subsubsection{Thromboprophylaxis Protocols}

Most participants (19,76.0\%) indicated that their institution(s) had a thromboprophylaxis protocol, but only a little over half $(53.0 \%)$ had any processes in place to encourage adherence. After being provided with a summary of the NHMRC recommendations to review, participants were asked if there was anything they felt was not appropriate in the recommendations; most surgeons (20, $80.0 \%$ ) responded 'yes'. The two most common reasons cited (as described in a free-text box) were assertions that the recommendations were grounded on an inappropriate evidence base, and did not include aspirin as an option for sole thromboprophylaxis (Table 25).

Table 25. Why the NHMRC recommendations were deemed inappropriate $(\mathrm{N}=20)$.

\begin{tabular}{|l|l|l|}
\hline Theme & $\mathbf{n}$ \\
\hline Inappropriate evidence base & 11 \\
\hline Aspirin should be included as a sole agent & 6 \\
\hline Aggressive mobilisation is not included & 30.0 \\
\hline Industry funding biases literature and hence guidelines & 3.0 \\
\hline Inappropriate duration recommended & 4 \\
\hline Bleeding risk is an issue & 30.0 \\
\hline No orthopaedic input into developing guidelines & 15.0 \\
\hline Heavy reliance on pharmacological prophylaxis & 3 \\
\hline Warfarin should be included as a sole agent & 3 \\
\hline
\end{tabular}




\subsection{Discussion}

The aim of this survey was two-fold: to identify prescribing trends and to identify enablers and barriers to prescribing from the perspective of the orthopaedic surgeon. The first part of the survey identified that prescribing preferences vary between surgeons. The cases identified however that almost all participants were inclined to change and tailor their prescribing according to their patient's associated risk factors.

While some participants' preferred prophylaxis regimes were aligned with the NHMRC Guideline, approximately half of them were not. Prescribing was less likely to adhere with the guideline if participants were concerned about bleeding, perceived pharmacological prophylaxis to be no more superior to mechanical prophylaxis or perceived VTE risk to be too low to warrant prophylaxis. These concerns and perceptions can be regarded as barriers to prescribing pharmacological prophylaxis. Surgeon prescribing was more likely to be adherent with the NHMRC Guideline when they were prescribing for a patient had a history of VTE; this was also identified as a factor in the first part of the survey that would prompt prescription of pharmacological prophylaxis (Table 23).

The results of this small survey confirm that the provision of VTE prophylaxis is variable, as indicated by other clinical audits, including those in the preceding chapters. ${ }^{17}$ Interestingly, whereas the results outlined in Chapters 3 and 4 were subject to being influenced by other doctors' prescribing preferences (e.g. junior doctors and geriatricians), this survey confirmed that surgeons are responsible for at least part of the prescribing variability identified in studies.

Despite the absence of proven strategies for CPG implementation, it is tempting to blame surgeons for the guideline-practice gap. ${ }^{140}$ One could argue, however, that guideline-practice gaps that persist over many years (as the thromboprophylaxis one has in Australia) are symptomatic of deficiencies in either the guidelines themselves or their implementation strategies, and not clinicians' aptitude. ${ }^{10}$ This is evident when one considers that many CPG have not been fully implemented into practice, despite widespread acceptance of the value of evidence based recommendations by the health community. ${ }^{140}$

The strength of CPG lie in their ability to summarise scientific evidence that is available for decision making, whereby the outcomes are either black (harmful: harms far outweigh benefits) or white (beneficial: benefits far outweigh harms); recommendations with insufficient evidence or risk-benefit ratios that are very close are termed grey. ${ }^{178}$ As highlighted in Chapter 2, there have traditionally been marked differences in thromboprophylaxis recommendations between guidelines, particularly regarding the appropriateness of prescribing aspirin and warfarin as sole agents. ${ }^{171}$ Recommendations in these areas have in some cases been potentially influenced by expert opinion, and could arguably be classified as grey. ${ }^{68,139,198}$ Another point of debate is the appropriateness of extended-duration prophylaxis, and the evidence (or the alleged lack thereof) to 
support it. This is apparent in the 2011 AAOS Guideline which does not recommend any specific duration of prophylaxis therapy for arthroplasty patients, instead stating that "In the absence of reliable evidence about how long to employ these prophylactic strategies, it is the opinion of this work group that patients and physicians discuss the duration of prophylaxis. ${ }^{68}$ That the surgeons participating in this survey varied in their choice of preferred pharmacological agent and duration is likely a reflection of the ongoing uncertainty and debate in these areas, and it is not surprising then that the practice gap continues to persist.

Although few surgeons believed pharmacological prophylaxis prevents fatal PE, and many responded that VTE risk is low following arthroplasty, all participants reported routinely prescribing pharmacological prophylaxis postoperatively, with similar inclinations for LMWH and aspirin as described in Chapters 3 and 4. One of the main arguments for the use of aspirin postoperatively is its lower bleeding potential compared to anticoagulants. ${ }^{50,145}$ Most of the surgeons who preferred aspirin in this study also believed that pharmacological prophylaxis is not safe as it increases bleeding risk. One of the main arguments for the use of anticoagulants is that they reduce VTE more effectively than aspirin. Interesting, the three surgeons who perceived pharmacological prophylaxis as effective in preventing fatal PE favoured an anticoagulant postarthroplasty.

In addition to bleeding concerns and a perceived low VTE risk postoperatively, a strong barrier identified to pharmacological prophylaxis use was the belief that study evidence is not applicable to real-world populations. This may be because some contemporary guidelines have cited papers that have used asymptomatic VTE as endpoints, the clinical relevance of which is controversial. ${ }^{139,171}$ Furthermore, trials often exclude many real-life situations of patients e.g. those with extreme body weights, significant renal impairment or multiple comorbidities.

As all participating surgeons were ASA members, it was anticipated many would be familiar with the ASA Guideline. That so many participants (30.0\%) had only heard of the NHMRC Guideline in passing was unexpected. Also surprising was the large proportion of participants who deemed the NHMRC Guideline inappropriate. Interestingly, all surgeons who reported not being familiar with the NHMRC Guideline deemed it inappropriate once reviewed; two thirds of surgeons who were familiar with it agreed. Also interestingly, many surgeons (40.0\%) who questioned the appropriateness of the evidence base of the NHMRC Guideline stated earlier in the survey that they believed study evidence pertaining to thromboprophylaxis is not applicable to real-world populations. Although all surgeons expressed familiarity with the ASA Guideline, their prescribing was not always consistent with its recommendations. This may be due to reservations regarding the ASA recommendations.

On six of the occasions when participants 'prescribed' an inappropriate dose for one of the case studies, the dose was excessively high and perhaps due to confusion over agents' generic and brand names; for example some surgeons prescribed rivaroxaban 40mg, fondaparinux 20mg (NB: 
only generic names were used in the survey). This suggests that any interventions to improve guideline adherence should highlight appropriate anticoagulant dosing requirements, particularly for the newer anticoagulants.

Although collaborating with the ASA did assist in targeting orthopaedic surgeons who regularly perform joint replacements, professional endorsement of surveys has also been reported to negatively affect response rates, and may be one reason why the response rate for this survey was so significantly lower than the reported $61 \%$ average from mailed physician surveys. ${ }^{199,200}$ As the invitation to participate in the survey was distributed electronically by the ASA, it was not possible to use the strategies employed in Chapter 5 to improve the response rate (namely personally signing each letter, adding a thank you note and including a hard copy of the survey in the invitation letter). An alternative approach could have been to increase the number of reminders: Braithwaite et al increased the response to their electronic survey of general practitioners from just under $30 \%$ to $52.4 \%$ following five electronic reminders (and up to $63 \%$ following a telephone based survey of non-responders). ${ }^{193}$ Unfortunately, the number of survey reminders sent for this study was capped by the ASA, and privacy laws prevented the ASA sharing their members' details for a telephone-based survey of non-responders.

It has been demonstrated that as response rates decrease, the risk for nonresponse bias increases. ${ }^{201}$ The response rate for this survey $(25.0 \%)$ was arguably low. Nonresponse bias is particularly relevant when the topic is considered sensitive. ${ }^{202}$ Thromboprophylaxis prescribing following hip and knee arthroplasty is arguably a sensitive topic, with surgeons vetoing the multicentre study in Chapter 4 because of its auditory nature and Molnar et al describing prescribing practices being influenced by the fear of litigation. ${ }^{121}$ It is possible then, that nonresponders may have been even less likely to comply or agree with thromboprophylaxis guideline recommendations than responders; however, only a follow up with non-responders would be able to confirm this.

Despite the limited response rate, the survey was successful in identifying many barriers against the smooth adoption of any thromboprophylaxis guideline including: doubt regarding thromboprophylaxis efficacy, a perceived low VTE risk, and belief that recommendations are based on inappropriate evidence. 


\section{Chapter Seven: Health Professionals’ Perceptions}

\subsection{Introduction}

The surveys in Chapters 5 and 6 explored enablers and barriers to thromboprophylaxis prescribing; however they did so in a limited way because surveys are by design a one way conversation with no opportunity to explore ideas further. To overcome these limitations, a qualitative arm was added to the research to more fully explore the perspectives and experiences of health professionals involved with the provision of thromboprophylaxis to arthroplasty patients.

\subsection{Aim}

The aim of this study was three-fold:

- to explore factors that drive and influence thromboprophylaxis prescribing postoperatively;

- to explore surgeons' position and point of view; and

- to explore what factors surgeons' identify as important, and why.

\subsection{Methods}

The qualitative approach selected for this study was informed by grounded theory. This approach is inductive and allows theory to be developed through an iterative analysis of the empirical data. ${ }^{203}$

An iterative method is one in which the process of data collection and analysis are both cyclic and occurring simultaneously so that both processes are regularly reviewed and revised. ${ }^{204}$ The grounded theory approach consists of a precise set of steps that are carefully executed in a particular order. For this project a modified version of grounded theory was adopted that included the following features: purposeful sampling; an iterative data collection and analysis process whereby data was analysed as it was collected and the insights gained through this process were used to shape the subsequent data collection and analysis; the use of coding for data analysis; and most importantly, the commitment to an inductive approach that allowed theories to emerge from the data.

\subsubsection{Recruitment and Sampling}

The sampling process in grounded theory research (also widely used in qualitative research more generally) is termed theoretical or purposeful sampling. It involves purposefully selecting participant groups and data sources for their ability to help the research to meet the aims of the 
study and as the study progresses, to provide data that can confirm, challenge or expand an emerging theme. ${ }^{204}$ The primary aim of this study was to explore factors that drive and influence thromboprophylaxis prescribing post-arthroplasty. To gain an understanding of the decision making process and experiences, surgeons were approached directly. To complement these views and to get a better grasp of real practice, these interviews were supplemented by interviews with junior orthopaedic doctors, general practitioners and clinical pharmacists (orthopaedic pharmacists and pharmacists involved in the formulation of a Tasmanian thromboprophylaxis guideline for hip and knee arthroplasty patients).

An invitation to participate in the interviews was sent to all orthopaedic surgeons practising in Tasmania (eighteen surgeons); two geriatric consultants (who practise in orthopaedics) and six junior orthopaedic doctors at the local public hospital (the RHH); five clinical pharmacists; and five orthopaedic surgeons practising in Victoria who expressed interest in participating in the study after completing the online survey outlined in Chapter 6. Additionally, invitations were sent to 186 general practitioners (GPs) practising within a half hour driving radius to the main hospitals in Hobart. In order to maximise participant uptake of the interviews, participants were offered faceto-face interviews at a time and place convenient to them, as well as a AUD200 gift voucher (GPs only) or an opportunity to enter a draw for a second generation Apple iPad (valued at AUD629, prize draw open to everyone else).

A purposeful sample of eleven orthopaedic surgeons and two junior orthopaedic doctors who worked across nine hospitals was obtained, as well as interviews with four clinical pharmacists and five GPs. The hospitals where participants worked included five public and four private hospitals, predominantly in Tasmania. All interviews were conducted over a three month period from April to June 2011.

\subsubsection{Interview Style}

Semi-structured interviewing was selected for the interview style as this approach is highly suitable for a study informed by a grounded theory approach, and because it allowed information to be collected in areas of interest that were identified in the online surveys, whilst still including a significant degree of flexibility to adjust the order, wording and type of questions asked during the interview. This flexibility allowed new topics and themes to be explored as they emerged, thereby facilitating in-depth understanding. ${ }^{205}$ The interview schedules generally consisted of open ended questions to which participants were encouraged to respond freely (see Appendix D).

The interviews were conducted one-on-one between the researcher and each participant. The use of focus groups was considered briefly; however, there were ethical challenges identified (related to confidentiality and anonymity) that had the potential to inhibit discussion. It was considered more appropriate therefore, for the participants to be interviewed separately and in private; this provided them with the opportunity to express their views fully, and to openly relate 
their experiences and opinions of other peoples' practice without being influenced by other participants in a large focus group. One-on-one interviews are also usually readily accepted by people. $^{203}$

With the exception of one interview, participants were interviewed in their work spaces, thereby allowing the researcher to observe them in their working environment. Before each interview commenced, each participant's permission was sought to allow the interview to be recorded on two digital voice recorders. Once full consent was confirmed a few background details were noted and the interviews commenced. At the end of each interview, participants were provided with the opportunity to clarify any comments made, to speak on any other topics and/or to ask any questions of the researcher. Each participant's permission was obtained to contact them after the interview so that any necessary clarifications could be made and any pertinent topics that emerged in subsequent interviews could be explored.

Throughout the interview, the researcher took steps to build rapport through active and empathic listening. Directly following each interview these observations were audio recorded, along with initial reflections of the interview (these were later transcribed and hereafter referred to as field notes).

\subsubsection{Data Analysis}

In line with the iterative study design, the principle of constant comparison was adopted in the data analysis. This involves comparing each piece of data with every other piece of relevant data and is important in developing theories that are grounded in the data. ${ }^{52}$ By employing this process, cycles of data analysis were intertwined closely with the data collection phase, and the result of the ongoing data analysis informed subsequent data collection. ${ }^{52,204}$ The cycle of comparison and reflection on 'old' and 'new' data was repeated several times.

All interviews were transcribed verbatim by the researcher into text. Transcription, the first 'official' step of data analysis, was always combined with reflection and review of field notes. Questions arising during the initial transcribing process were noted and explored in subsequent interviews and in limited email correspondence with the participants. In this way it was possible to answer questions that arose from the analysis of and reflection on previous data.

The coding process was conducted using an iterative thematic analysis approach, informed by grounded theory and constant comparison. As interviews were transcribed they were coded, meaning that interesting and important sections such as concepts, observations, reasons given for prescribing practices, comments on guidelines etc. (anything relevant to the research questions) were marked and labelled. As the research progressed, new codes were developed and earlier interviews were reread and compared to the newer interviews.

Subsequently, 'axiel coding' was performed where these initial codes were grouped into thematic categories. A simple 'copy and paste' technique was used to group coded sections from 
the transcripts together under relevant thematic categories (which grew and broadened with each transcript review).

The thematic categories were then reviewed, reduced and expanded through a process of constant comparison and then revised and refined into broader themes until they appeared to be saturated. Finally, after these preliminary findings had been established, all the interview transcripts were reread to fine tune the analysis, and ensure nothing had been missed. Eighty-two categories and themes were identified in the data in total. These themes are summarised under twenty-two subheadings in the following sections.

Another researcher from a different background (sociology) read through the analysis several times to offer a second opinion and promote reflexivity. They agreed that the final thematic categories described in the following pages reflected the source data.

\subsection{Results}

\subsubsection{Participants}

The surgeon participants (S) were all Anglo-Saxon males, with varying degrees of orthopaedic surgery experience between them (6 to 23 years, average 13 years). The surgeons estimated that they conducted between 12 and 16 joint replacements a month each, with a slight predominance for knee arthroplasties, reflective of the national trend, and data previously described.

The GPs were all Anglo-Saxon; two were females, three were males. Between them they had 6 to 35 years of practice as a GP (average 21 years). The GPs were unable to estimate how many of their patients underwent hip and knee arthroplasties each year, but described them as 'coming in groups'. One GP said she had not had any patients undergo the surgeries for many years, but had patients on waiting lists for joint replacement(s).

The junior doctors (JD) were both young Middle Eastern males and had practised medicine for less than one year, and on average only 5 weeks in orthopaedic surgery (10 weeks is the normal intern placement). The clinical pharmacists (CP) were of Anglo-Saxon background; one was a female, three were males. Between them they had 6 months to 22 years of practice as a registered pharmacist practising in clinical pharmacy.

\subsubsection{Surgical and Postoperative Changes}

When asked if the surgical procedure had changed significantly over the years, one surgeon stated that 'the operation is basically the same', while another said he had been using a more minimally invasive approach in recent years that allowed quicker, less painful procedures with a potentially lower complication rate. Surgeons generally remarked that there was a trend to 'operate on younger individuals than before', 'fitter patients' and not 'elderly patients with multiple 
comorbidities'. Other factors that surgeons noted had changed over the years included the length of stay in hospital (trending downwards), an increase in the use of mechanical thromboprophylaxis, the introduction of bedding exercises and, importantly, the elimination of any preoperative anticoagulant doses. Finally, many surgeons spoke of early mobilisation as being the 'biggest factor' that had changed over the years.

The patients used to be admitted the day before, lie around all day, sometimes rest in bed for three or four days, plus after surgery. Now it's 'get the patient out of bed and get them mobile'. Quicker operations, shorter hospitalisation times. S5

One surgeon noted that as a trainee, VTE 'was quite a common thing'; however, he felt that the increased attention given to VTE prevention in recent years had led to many different care changes (including thromboprophylaxis use) which had reduced VTE incidence overall.

\subsubsection{Prescribing Patterns}

Junior orthopaedic doctors said their prescribing was directed by more senior doctors.

In terms of postoperative orders, there's normally a kind of protocol to follow. In terms of anything outside of those orders then there is flexibility of prescribing. But generally your prescribing is led by the, the geri[atric] consultants. ... There's no protocol. It's more actually determined by the surgeon. JD1

The junior doctors described most surgeons as prescribing identically; however, when the surgeons were interviewed they described a different picture. This is likely because both junior doctors practised at one public hospital, whereas the surgeons practised across many different hospitals and sectors.

Despite the relatively small number of surgeons interviewed, their prescribing patterns differed considerably, both in agent and duration. Some surgeons prescribed anticoagulants during the inpatient stay and an antiplatelet (aspirin) at home for up to 6 weeks. One participant prescribed aspirin solely for 6 weeks postoperatively. Others prescribed anticoagulants (LMWH and rivaroxaban) for up to 6 weeks postoperatively. Notably, rivaroxaban was only being used in the context of a study being conducted at one of the public hospitals; that is, none of the surgeons were voluntarily prescribing it.

General practitioners said they did not independently prescribe anything for their patients post-arthroplasty unless directed to by the operating surgeon. Although pharmacists do not prescribe thromboprophylaxis themselves they said they encouraged doctors to use guidelines in practice, (particularly the ACCP Guideline) as part of their role as clinical pharmacists.

I think some, some doctors are very receptive. I think it generally depends on the intern and how much they're, how happy they are to prescribe, because again the registrars don't generally mind too much what happens after the surgery, um so they sort of will go, you know, if the intern's 
happy to do it, they'll do it. Sometimes the consultants have strict views, and that determines what's done. But yeah. Most of the time's not too bad. CP3

\subsubsection{Factors that Influenced Prescribing Patterns}

There were many factors that were found to influence prescribing patterns amongst participants (described in detail in proceeding sections). Although prescribing patterns were different between surgeons, they agreed that first and foremost each patient should be treated as a whole person, balancing the risks and benefits associated with pharmacological prophylaxis, and taking advantage of non-pharmacological means of VTE prevention.

Basically you've gotta treat more than just the heart, or more than just the lungs, or more than just the clots, or more than just.. You know you have to stop yourself from being too tunnel visioned about what you're trying to do. S6

Well I think it's more than just a prescribing pattern. There are things which we do at the time of the operation; so it's my preference to have a spinal anaesthetic which reduces the risk. I use calf compression on the leg which is not being operated on. I use foot pumps postoperatively. And then the routine prophylaxis is to use clexane afterwards for both hips and knees. S4

This sentiment was echoed by GPs.

We've got to remember that we're treating the patient. We're not treating ourselves. We're not blindly doing what we're supposed to be doing. We're doing things because it makes a difference to that individual patient. And we've got to know [that] what we're doing is relevant to that patient, and provides some benefit. GP1

I mean, I think probably that's probably a better guideline [AAOS Guideline] in some ways because you're actually.. rather than one size fits all it's a case of assessing the patient that's sitting in front of you. Because every patient that sits in front of you is going to be different. And that probably is a bit more I suppose, Um. You know, gonna, gonna suit the patient's individual circumstances better. Rather than just, just saying well it says here to give clexane. So that would probably be, yeah, I think that would be ok. GP2

\subsubsection{Patient History}

Most surgeons spoke of stratifying their patients to some degree before prescribing pharmacological prophylaxis, particularly anticoagulants. For example, five surgeons noted that if a patient had a personal or family history of VTE (particularly unprovoked VTE) they would 'investigate them further for thrombotic risk', 'tend to be more aggressive' and 'talk to them about the possibility of long term Clexane [LMWH]'. The duration of therapy prescribed for anticoagulants was described as varying 'according to clinical scenario', whereby the decision to extend anticoagulant cover is weighed up against other patient-centric concerns such as their 'frailty' and 'their ability to self-administer' injections. 
One surgeon said he altered his prescribing if he got 'the feeling' that a patient would bleed. Likewise, surgeons spoke of altering their prescribing for patients with a history of bleeding by withholding the first dose of prophylaxis until one day after surgery and/or using a smaller dose; however, this was described as 'rarely' being an issue.

\subsubsection{Training}

Surgeons said that their surgical training influenced their prescribing patterns, but only in combination with other influencing factors.

Look, some of it's training, some of it's my interpretation of the current literature. Some of it's discussion with peers at conference. $S 4$

Yeah a little bit of what's just been handed down. Uh there's a little bit of discussion with my colleagues, um and a little of the sort of, the review papers and things like that as well. All sorts of [things] put together. S1

\subsubsection{Outside Input}

Although the decision to prescribe thromboprophylaxis and the type of thromboprophylaxis was universally agreed to ultimately be the operating surgeon's decision, reference was also made by three surgeons to anaesthetists having input and involvement in decision making. Occasionally, patients and their families also have input into prophylaxis postoperatively.

...his daughter was a GP, he's a retired surgeon - did an ankle arthrodesis on him. I was happy to just have him on aspirin. His daughter insisted, as did another daughter who was a pathologist.. no a microbiologist, insisted that he go on to clexane. And so I said fine. S2

GPs stated that they did not instigate thromboprophylaxis for their patients after discharge, unless directed to by the operating surgeon.

So if they come out of hospital not on thromboprophylaxis, I don't usually start it because I presume they didn't think it was necessary. I defer to their expertise in that area. GP5

One GP said that he would prescribe thromboprophylaxis for any patients that were 'very high risk, such as diabetic, overweight and pretty well immobilised after their knee replacement'; however, it 'hasn't happened because those people are always on something'. Although GPs and surgeons stated GPs were not involved in thromboprophylaxis decision making, surgeons expressed openness for more GP input. Furthermore, both GPs and surgeons noted that GPs are the ones to treat clots that arise following discharge.

My specific involvement in anticoagulation afterwards [after surgery] has been sort of pretty minimal. Actually I'm just trying to think of anyone. I haven't been involved except with those which the surgeons give DVTs to. GP1 


\subsubsection{Literature and Study Data}

At different points during their interview each surgeon cited research papers to lend credit to their point. Some surgeons said they attended a 'journal club' to discuss relevant literature, and others spoke of discussing literature with colleagues in general or at conferences. Interestingly, participants expressed hesitation and scepticism in accepting study evidence at face value.

I've been in research long enough to know you can tailormake your result a bit as well. S7 So my colleague who's you know, keen on aspirin, he's always flashing these papers that prove, yet again that aspirin's just as effective as uh, low molecular weight heparin. ...if you wanna search far enough you can find some sort of study that'll support your argument. S8 Uh and as I'm sure you're aware the evidence, you know, for that [extended therapy thromboprophylaxis] is pretty soft. You know to actually say that they've prevented a pulmonary embolus is um [a] pretty hard thing to prove. S1

In general, GPs and surgeons remarked that papers that used asymptomatic VTE as endpoints had little clinical relevance or impact on their prescribing.

No. [laughs] ... They're using asymptomatic clots as a surrogate measure for risk of PE. And there is no relationship. There is no established relationship. So I think it's a waste of time to try and prevent those asymptomatic clots anyway. $S 2$

Yeah it's just bullshit. Exactly what's the point to that? ... To me, the important thing is pulmonary embolism and you know, symptomatic DVT, maybe, as a sort of lesser worry. And only above knee DVT is the concern, rather than below knee. S1

It has zero relevance because I believe if I look hard enough I will find a clot in every one of my knee replacements and every one of my hip replacement patients. S3

Although, you know, in discussing this with the haematologists you know, an asymptomatic deep vein thrombosis, unless you're thinking it's a totally different animal to, to a, to a, you know, a symptomatic DVT, is probably just this early stage of something bigger. Whether it progresses or whatever. It's probably just one end of the extreme. But um. I'm less inclined um, to look favourably on a study if the end point is asymptomatic DVTs. S10

Pharmacists tended to be more conservative in their remarks.

Oh I think it's part of an overall picture.... But I, I would think, I would personally put value in, in something that can be shown to, to reduce overall clot burden. But clearly, clearly, it is clinical endpoints that are important and so, you know, two drugs, one prevents asymptomatic or sub therapeutic clot better than other, yet hard clinical outcomes are the same. Well you've then gotta think about what that means and think about, think very seriously about, you know, other aspects, other components of the triad of, of, um, therapeutic, measure of therapeutic success. Clinical outcome, safety and cost. CP1

From other reading I've seen, it talks about not only the complications of PE, but long term, I think it's called, the post, post thrombotic syndrome, which some of the vascular. Some vascular doctors have sort of said, yeah long term you get like obviously decreased like, um, um, increased risk of getting ulcers in the long term, which those sort of things, um, um, if we can prevent those, 
they say that it's probably likely to improve their, their long term outcomes, and their vascular system. So I think they definitely should be looked at. So I wouldn't just say because the patient's not aware, it's not important. CP2

\subsubsection{Thromboprophylaxis Aim}

As described above, surgeons expressed little clinical concern for below knee DVT; similar remarks were made by GPs. In contrast, PE and symptomatic above knee DVT did pose a concern for many participants, and they described their prescribing as being geared to preventing them while balancing the risks associated with prophylaxis. Three participants spoke distinctly of trying to reduce fatal PEs, not simply PEs in general. Participants who spoke about trying to reduce overall mortality, instead of just VTE, spoke particularly favourably about prescribing antiplatelets.

And I defend aspirin on the basis of um, when I was training, then there were a couple of metaanalyses that looked at thromboprophylaxis. And what they did find was that there was a lower all-cause mortality in the patient group that received aspirin alone, compared to um those that received other thromboprophylaxis. So while my patients might not die of a PE, I'm lowering the risk for um stroke and heart attack. So that's my rationale for using aspirin. S2

\subsubsection{Local Protocols and Hospital Sectors}

Surgeons operating at hospitals with unit or hospital thromboprophylaxis protocols commented that their prescribing patterns were influenced by the existence of these protocols. All participants performed surgeries in both the public and private sector; however, unit protocols were only discussed in relation to the public sector.

Overall, surgeons were mixed on whether their prescribing practices differed between their private and public practice. Some surgeons said they prescribed identically in both sectors in order to keep it simple and to avoid 'a system that's so confusing that it's likely to lead to errors', whereas other surgeons practised differently between the sectors.

All participants who had practices that differed between private and public worked at public hospitals with unit thromboprophylaxis protocols in place which were adhered to by junior doctors. Participants who worked at a public hospital without a unit protocol spoke of liaising with registrars regarding their prescribing preferences early in the registrar's year of training. Interestingly four surgeons noted that they 'tend to be relatively ignorant of what's actually happening' in practice.

\subsubsection{Thromboprophylaxis Efficacy}

Doubt was often expressed regarding the effectiveness of pharmacological agents to prevent VTE. And I think that generally surgeons are divided into two camps like that. Those who really think it's [LMWH] a good thing, and those who think it's a waste of time. And then there's probably a 
smaller group who do it just because it's maybe medico-legally seen to be doing the right thing. S2

I'm not convinced that taking clexane [LMWH], warfarin or any other agent decreases the incidence of significant fatal PE. I'm not convinced that there's any agent that would reduce that risk. S5

I'm just not convinced the science is good enough to support its [aspirin] use. It seems that it's a gentler thing to do than to use heparin based treatments. ... But it might not be effective. S4

All surgeons who doubted the effectiveness of antiplatelets to prevent VTE did not prescribe them regularly. Notably, some of the surgeons who doubted the effectiveness of anticoagulants did prescribe them during the inpatient phase and chose to switch to aspirin on discharge. Interestingly, these surgeons were not always obliged by the presence of any unit or hospital protocol to prescribe anticoagulants during the inpatient stay.

Conversely some of the surgeons who spoke positively about aspirin's ability to reduce 'allcause mortality' did not prescribe it during the inpatient phase, potentially due to the presence of unit protocols that recommended against it.

\subsubsection{Patient Compliance and Satisfaction}

As described in Chapter 2, many longstanding anticoagulants require subcutaneous injections that are self-administered (or administered by a family member) if required after discharge; which can be inconvenient. Surgeons who did not prescribe anticoagulants cited this as one reason why they didn't.

Ah, well one is, I'm not convinced that a lot of patients are compliant with injections because they don't like injections. S5

They like not having to give themselves injections when they go home. And even having injections while they're in hospital. $S 8$

Conversely, participants who did prescribe them, although mindful that patients may not like self-administering injections, did not consider it to be an insurmountable obstacle.

Uh yeah, the patients do as they're told. Some of them don't like it. Some of them don't like injecting themselves. But it's not so big a problem that they say I'm just not going to do this. It's not that much of an issue. S6

I'm a little bit of a naïve believer of the, the good so to speak. I think that they [patients], they care for themselves. It's better. You have a better night sleep if you, you believe in the good I think. So I think they want to! S7

\subsubsection{Simplicity}

The desire to keep things simple and uncomplicated came up during the interviews on multiple levels. As described previously, some surgeons prescribed identically between their private and public practice for simplicity and to avoid confusion; however, there are many other examples of 
this desire for simplicity influencing practice. Contemporary guidelines typically recommend shorter courses of prophylaxis for knee patients than hip patients. Two participants acknowledged this but said they prescribed the same duration of therapy for both types of patients for ease and to reduce confusion.

Likewise, three participants spoke of prescribing medications either now and/or in the past that specifically did not require phoning up for authority, which 'takes five or ten minutes' and requires the surgeon 'to fill out these forms', which is 'a real pain'.

Participants who worked at public hospitals with multiple orthopaedic surgeons spoke of unit protocols being in place to be more practical and make things simpler, because if all surgeons 'insisted on having our own kind of foibles, then it's [sic] just make it too difficult for everyone to remember who does what', and it becomes 'too unwieldy'.

One participant noted that his actual practice 'differs slightly' from what he considered best practice for simplicity's sake.

I give clexane whilst the patient is an inpatient. And I give all patients information that when they go home they go on to thirty five days of aspirin. ... My best practice idea would be to have a fixed duration of clexane [LMWH] for all patients but it would probably be no more than 10 days and then maybe an oral agent after that. S3

\subsubsection{Contemporary Guidelines}

Although guidelines did influence prescribing, their use was usually discussed in combination with other influences, in particular clinical judgement.

The same rule doesn't apply to every patient. Like again, you've got a skinny old lady who bleeds like a stuffed pig when you cut her open; you have to adapt. You can't just 'the guideline says this, I have to do that. ...Look I think a recommendation is fine. And a guideline, but I think you have to taper your treatment protocol to the individual. So you can't - the same rule doesn't apply to every patient. $S 5$

GPs expressed similar sentiments.

It's all very well having a protocol but you've actually got to think critically in following that protocol and make sure that it's relevant to your individual patient. GP1

In some instances participants expressed concern that guidelines in general created medicolegal concerns, particularly if they make blanket recommendations.

I'm shocked. The guideline states that any patient being immobilised, for example with ankle fracture, should have prophylaxis for the whole immobilisation time, which is at least 6 weeks and maybe more. And I don't understand what you're going to do about this. It's very clearly written down. And, and I don't know. I tried to bring this up, a couple of times: what I was officially supposed to do about it, because it's sort of written down. ...So that, that is [a] medicolegal concern...the state would have to pay a lot more money, and maybe that should be a 
concern too, but that doesn't come to my heart so much as if I would be legally bashed up or something. 57

Other participants found guidelines useful and reassuring.

I think that for the medico-legal reasons most of us want to know what's the middle of the road standard care. I mean over anticoagulating people and having increased bleeding risk, and under coagulating people and having increased exposure risk to death from PE. S4

One participant expressed a strong sentiment that the literature and guidelines are not well balanced, feeling that guidelines are being 'driven by the drug companies' because there are professors sponsored by drug companies on some guideline boards. The participant also remarked that there's a 'vested interest for a lot of these drug companies to get their drugs on the market.'

Remarkably, the only surgeon who described having successful experience with thromboprophylaxis guideline formulation was the only one who noted that the NHMRC Guideline incorporates a step-by-step guide that encourages the surgeon to consider 'the patient's clotting risk, the patient's bleeding profile', their surgery, and that it requires the surgeon to 'make a conscious decision' on thromboprophylaxis requirements for each individual patient.

\subsubsection{Medico-legal Concerns}

Although surgeons and GPs were mindful of medico-legal considerations, they were never described as the foremost concern when prescribing thromboprophylaxis; priority instead was given to clinical grounds in the decision making process. It was noted that this may be because medico-legal exposure in Tasmania 'is not as bad' as it is in other states and territories.

I like to think that my primary concern is to do what I think is best for the patient rather than what some lawyer might pick me up on, or if I'd have to you know, argue my case in court. Uh so, for me, it's, it's not a strong consideration. $S 8$

I never let it worry me too much. Yeah, we have medico-legal you know, coverage, you know, pay your insurance, and if you have a problem you hope that they sort it out for you. S1 I'm just interested in preventing complications for the patient really. Medico-legal doesn't really come into it I think, not for me anyway. GP3

Nonetheless, one surgeon did note that 'there's a vast spectrum of [different] individual [patients], and most of us are trying to say we want to sail that line without exposing ourselves to medico-legal threats as well'. Furthermore, as expressed in 7.4.4.10 Contemporary Guidelines there was some concern expressed that rules and regulations in Guidelines could impose a medicolegal concern.

\subsubsection{Culture}

One participant noted that the culture and systems in Australia are different from those experienced overseas, describing Australia as being a country with a great 'listening ear for the individual 
opinion'. In other countries the surgeons were described collectively as 'more of a public work force where the individual opinion was probably less listened to than here - here, each consultant is more an individual with his or her needs or interests'. In other countries 'there were no questions about it', you do what the guidelines recommend; 'but here, you can see all [degrees of] variance'.

\subsubsection{Complications}

Both VTE and bleeding complications were cited by surgeons and GPs as major factors that have influenced their practice. In a few instances either personal or anecdotal experience of increased wound ooze and bleeding with anticoagulant use was enough to deter surgeons from prescribing them. A GP said her opinion and practice changed after one of her patients was left a paraplegic following a postoperative spinal bleed; however, she described later on in the interview feeling 'a bit conflicted at the moment' about what to do with thromboprophylaxis following surgery. When asked what had changed in her opinion and practice she described taking greater initiative in liaising with the surgeon and anaesthetist, in addition to changes she had made to her prescribing which expressly oppose all current recommendations for warfarin (re-)initiation.

And perhaps I'm a bit slower in getting the warfarin back on. So I give them a little bit longer so they actually, probably when they get home, I don't, I don't continue them on the clexane [LMWH], I just, unless they've got a really good reason. ... what I have done is I don't continue the clexane [LMWH], I just start the warfarin slowly and build it up. Rather than put them on the clexane [LMWH]. ...before then I was continuing the clexane [LMWH] until they reached the therapeutic INR. GP2

Conversely, a surgeon spoke of being 'pretty much a doubter' when it came to thromboprophylaxis, until one of his patients experienced a fatal VTE.

You know, I've had one patient many, many, many, MANY years ago die of a [PE], after a hip replacement. I was actually on the ward at the time. It was two days after, I remember that. I operated on Thursday and it happened on Saturday morning. I was doing a ward round. I couldn't believe it. I'd just seen her, and she looked fantastic. And then a little bit later you know, they were calling the code blue and um, and um, I walked around and then, all these intensive care people trying to resuscitate her. It was dreadful. S10

When asked how this experience had affected his prescribing, the participant spoke of it being a trigger to become more concerned about VTE prophylaxis, and becoming 'involved with it for a while'. Interestingly he noted that his subsequent involvement with thromboprophylaxis guidelines had led him to become 'totally convinced' of the 'benefit of extended prophylaxis', and 'a firm believer' that the benefits of using 'mechanical prophylaxis is additive to that'.

In order for VTE complications to influence practice, however, operating surgeons must be aware of their (and their colleagues') complication rate. Interestingly, surgeons said that even though they 'like to think' they are well informed of all postoperative complications, there's 
'probably quite a few that slip under the radar'; which means they 'don't see a huge number [of VTE] in practice'.

You know, I think the patients would tell me [about a DVT or PE out of hospital]. And I don't hear about it. $S 1$

Now I don't see a lot of the DVTs, PEs. I know quite a few are managed by GPs and physicians [which] we don't find out about it [sic]. S5

And often, I shouldn't say often because it doesn't happen very often. But not infrequently if a patient does present with a symptomatic DVT or a PE, there is actually no notification given to the operating surgeon, or even the operating team. So in other words, we will occasionally find out via the grapevine, or at a 6 week check when our patient comes back in that they're on warfarin for a PE. S3

\subsubsection{A Statewide Guideline}

In recent years the creation of a statewide thromboprophylaxis guideline for Tasmania has been proposed. There was a mixed response to this topic in the Tasmanian interviews with some participants feeling that it's not 'going to add a lot more - really there's recommendations from a lot of very well qualified committees already', and others conceding that such a guideline 'is fine' so long as surgeons are able to 'taper the treatment protocol to the individual', or you're 'kind of leaving yourselves open a bit to, you know, people saying get stuffed!'. One participant spoke of the push for a statewide guideline as 'typical DHHS' behaviour, wanting something that 'would be seen as the due standard of care' and that any surgeon that deviated from it 'would have to explain why' (NB: DHHS = Department of Health and Human Services). He described the statewide proposal as 'a push for a policy', but that it was not 'a push from the orthopaedic surgeons themselves'. He went on to comment that 'it would have no formal impact on private surgeons' approaches'.

Having said that, I know myself and other surgeons would probably read it once, ignore most of $i t$, and if there was seen to be any support for our own practice or own preference, it would be quoted as that. ... So again, it's one of those things where you'd take what you want out of it. And ignore the rest. $S 3$

There were two participants who specifically commented that they felt orthopaedic surgeons must be part of the development committee of such a statewide guideline, as they are the "people who are on the ground, working that space', whereas 'a lot of the time the panel will be you know, physicians, and, and pharmacists, and people who are not at the coalface so to speak'.

GPs on the whole spoke positively about a statewide guideline, noting that it protects both the patient and the doctor, but that they must have a 'tier of assessment' incorporated into them, rather than being 'just a one size fits all'.

I think the sticky thing is they probably won't all agree. I think that maybe it may be better to say well look this is it, if you have any specific variations that you'd like to follow through, then you 
can make those sort of slight changes and sort of indicate on the sheet that that's your amended preferred protocol. So that covers the people that feel very strongly about something. GP1

\subsubsection{Novel Oral Agents}

Uptake of NOA was mixed and surgeons spoke of many reasons why, including: lack of knowledge about the new agent(s), concern and hesitation to start using a medication with little post-marketing surveillance data, and finally bleeding concerns arising from study data as well as personal and collective experience.

Um and then I guess it's a little bit of you know, fear of the unknown. ...there is obviously quite a bit of information about it [rivaroxaban]. And uh, you know quite a few studies, but obviously nowhere near the experience that we've had with uh, you know the sort of more standard ones. And uh, so people are still a little bit anxious about uh, how it's going to work out. S8

I think that [if] possible, it [rivaroxaban] needs some real-world use before I'm comfortable to use it. 54

When I was there 6, 7, 8 years ago, there was an introduction of an oral anticoagulant that went through the system and started to be used, and it was drawn back due to liver affections [sic]. So a bit of conservatism. $S 7$

I was using it [rivaroxaban] and I had a couple of patients that bled, so I've stopped using it. S5

I've heard anecdotally that there's increased risk of bleeding [with rivaroxaban]. S6

Interestingly three participants said government authority requirements put them off prescribing the new agents, describing the process of getting approval as 'a real nuisance', 'a real pain' and 'a deterrent' to using the new agents. Lastly, one participant commented that changing his practice to prescribe one of the new agents would mean changing the written documentation he gives to patients, and so it is 'partly apathy' why he doesn't trial them. Feeling apathetic was not limited to prescribing new agents or to this one participant: although many surgeons operate at the hospital this participant works at, he said between them 'they probably wouldn't do one ward round a fortnight'.

Lastly, surgeons were resistant to change because they felt generally content with current thromboprophylaxis options.

And also, sort of, to my knowledge, there hasn't been any real, sort of, problems to the clexane regime that we have. $S 7$

I guess we're all a bit conservative in, in that regard and you know, if something's working ok with what we're doing it's sort of, hard to change. ... So you need, you need a good reason to change I guess to, to take, take a, take a small risk that you know, the new thing is not going to be as good as it's cracked up to be. $S 8$

Interestingly, one of the GPs stated that one of the big benefits of warfarin therapy is that the regular blood tests involves 'monitoring this higher risk group a bit more closely', and that 'other 
things crop up' during the reviews. Nevertheless, it was noted by a surgeon that the new oral agents 'would make life a lot easier', so long as 'the benefit is higher than the risk'.

\subsubsection{Aspirin}

As noted in 7.4.4 Factors that Influenced Prescribing Patterns, participants were divided into two camps when it came to prescribing anticoagulants. When discussing aspirin use for VTE prophylaxis, surgeons were equally divided. Comments against aspirin use included 'that it's not appropriate', that not all surgeons were 'convinced the science is good enough to support its use', that it has 'dubious literature support', is 'less effective' than the mechanical options on their own, and that 'it seems to be a case of doing something to be seen to be doing something'. One participant who stated that aspirin is not appropriate conceded that aspirin might be somewhat effective in preventing VTE.

My background is that aspirin is good for the arterial side of the, the vascular tree, and, and uh, not much to do with clots. On the other hand it's a circle isn't it, so if you affect one end of it you might have an effect on the other end. 57

Conversely, participants who used aspirin commented that aspirin has good evidence to support it, citing 'equally low rates of DVTs', and frequently made reference to bleeding complications associated with anticoagulants. These participants also cited lower all-cause mortality in patients prescribed aspirin compared to other agents, with one surgeon stating that he is not using aspirin 'just to be seen to be doing something'.

As noted in 7.4.3 Prescribing Patterns, some participants made use of both anticoagulants and antiplatelets; typically anticoagulant cover in hospital and aspirin on discharge. Two of these participants said they prescribed aspirin after discharge because 'patients don't like giving themselves injections', with one conceding he would prescribe an oral anticoagulant (such as rivaroxaban) at discharge if he was convinced it would not cause more bleeding than his current inpatient preference, LMWH.

GPs were also divided on the efficacy of aspirin to prevent VTE. Some felt that it had no significant role to play while others felt it was better than nothing; or that it is an effective agent in patients who are mobile, but not those who are bedbound and have other comorbidities. One GP said that he did not use aspirin very often as 'it's not that effective, and also it's got the risks of gastric irritation'.

\subsubsection{Bleeding and Infections}

Concern was expressed by all participants regarding the potential of anticoagulants to cause excessive bleeding.

By definition it's an anticoagulant, so uh, um, you know it's got a good effect in that it decreases the risk of clotting where we don't want it. But it also decreases the clotting where we do want 
the clot. So you've gotta find that balance between not over anticoagulating versus you know having some effect and reducing the uh risk of venous thrombosis. $S 8$

Notably blood loss was not in itself the main concern for most participants, but rather its perceived propensity to cultivate infections which were described as being 'disastrous' by two surgeons and 'an absolute nightmare' by a third. A fourth told his patients that if they have an infected joint replacement 'they've lost a year of any productive time.' Another participant spoke of blood as an 'irritating thing in the tissues' that increased swelling and pain for the patient.

Uh, infection is another, very potentially serious complication ... if patients do get a haematoma, uh you know wound haematoma, then that definitely increase the risk of infection because haematomas are like the perfect blood clot, culture medium for bacteria. Uh and even with a discharging wound, you know if from a haematoma, the stuff that's trickling out you know that's sort of keeping the wound open, so the bugs on the surface can sort of sneak in and infect the whole thing. $S 8$

In this context, it was acknowledged by only one participant that medications are simply one factor that increases bleeding risk postoperatively. Other factors identified include: how big and long the operation is; the surgeon's skill; the invasiveness of the approach made; patient risk factors; and anything that happens perioperatively that may inadvertently increase bleeding. This same surgeon also noted that there are many factors that affect infection risk including patient factors (e.g. ability to fight infection), perioperative factors (e.g. skill of surgeon, sterile techniques of surgeon and scrub nurse, theatre sterility and filtration flow) and postoperative factors (e.g. patient nutrition and wound care).

\subsubsection{Mechanical Thromboprophylaxis}

Most participants commented on the importance of mechanical thromboprophylaxis in clot prevention, one stating he thought 'everyone should be on it'. However these positive comments were almost completely reserved for IPCD, and not GCS. Many participants (surgeons and GPs) commented that GCS were not effective, and/or were not tolerated very well by patients. A junior doctor added that he'd had patients slip and fall because of them; a fall can be potentially disastrous for any patient.

I receive phone calls occasionally from patients saying 'look how long do I have to keep these things going. I find them difficult to put on, or they annoy me, or they're hot.' And I say 'forget about them, don't worry about them'. S2

...of course the big problem's getting them [GCS] on, getting them off; or getting someone to help them take them off. So that's uh, that's the problem with it. GP1

They're hard to put on. Especially in that age group. You know because they're elderly and they've already got osteoarthritis. GP2 
Some patients hate them [GCS], some don't find them too difficult. It depends a lot on how frail they are and how much problem [sic] they have putting them on or off. Whether they have someone in the house who can help them. There's a whole lot of issues. GP3

Two participants said they used GCS 'for swelling control if there's a problem, rather than prophylaxis'. Another participant noted he used mechanical thromboprophylaxis 'more so because of hospital expectations and hospital policy', and that he is 'recently questioning it'.

Stockings I think are a waste of .. not a waste of time, but I think they're probably more problem than what you get from them. Whenever you use things you've gotta weigh up the risks versus the benefits. And I think the problem with stockings is that they are hard to get on and off. They tend to roll down and act as a tourniquet. 66

One surgeon who did speak positively about GCS (referred to in the interview as thromboembolic deterrent stockings or TEDs) expressed frustration that they were not always provided in a timely fashion to his patients.

Most of my patients who I've asked for longer length TED stockings didn't seem to have them two or three days later because they've had to be ordered from stores or that sort of thing. So I've almost given up on, on specifically prescribing TED stockings because it didn't matter what I wrote, or what I prescribed, even if 24 hours later I ask 'where is the patient's TED stockings?' I was told "oh we're on to it". And the next day they still wouldn't be fitted. So by day three or day four I'm .. it's just not worth it. S3

This same participant was the only one to mention any negative effects to using IPCD (referred to by the surgeon sequential compression devices or SCD).

But SCDs themselves have their own risks. I've recently had a nerve injury occur possibly as a result of the tubing on an SCD. So at what benefit in terms of DVT risk reduction or more importantly symptomatic DVT / PE risk reduction, at what cost do I then have complications with the device? S3

\subsubsection{Care Flow from Hospital to Community}

Although many surgeons described the care flow from hospital as being 'reasonable' or 'generally very good', GPs did not agree. They described it as being 'variable' with waiting periods of up to three years between the first referral and actual surgery, during which time many patient factors can change. GPs also said they were often unaware their patient had undergone surgery until the patient came in a week or so later.

Usually the patient is the one who fronts up and says I've had this done, and it's like you're working backwards sort of thing. And often, often it's the patient that's giving you the information as to what happened to them, and you have to then go chase up and find out if that's really the case. GP2 
Occasionally you might get a phone call saying 'Can you do this?' but just the quality of the process is, is, mostly abysmal. Occasionally we get something that's done well, but very occasionally and I think our expectations of the Royal are very low. GP1

When a patient is discharged from hospital, a discharge summary is sent to GPs to update them on a patient's wellbeing and discharge plan. GPs stated that discharge summaries took anywhere between 5 days to 6 months (if ever) to arrive.

And it's just, it just seems very primitive - seems like the Napoleonic era. Of course they didn't have faxes then, but they had fast horses. Maybe not much difference. GP5

Sometimes the patient arrives back before the discharge summary is received. Sometimes you just have summaries that are illegible. ... I think it really depends on the individual resident or intern and whether they get around to doing discharges in a timely fashion. GP3

In addition to this delay in receiving discharge summaries, GPs expressed dissatisfaction with their quality, describing them as being 'written squashed' and containing 'limited information' that ' $50 \%$ of the time I can't read'; and that while 'some of them are not bad, others are just a complete waste of time'.

Oh I had one recently where we had to keep calling them and telling them to redo it because it was hieroglyphics. I mean, you couldn't see it at all! GP2

On the one hand, discharge summaries from the Royal are usually inadequate, indecipherable, and you know, pretty useless; and there are medico-legal aspects - they're horrendous! ... GP1

One participant described the care flow from hospital to community being better in the private sector, but others did not notice any difference. When asked what areas could be improved, GPs stated that a 'good type written legible summary' with 'good clear written instructions' and 'a phone call from the discharging residents' would be 'ideal' so that there is 'open communication with the hospital'. One GP noted that in general practice, they're 'responsible for that patients care from then on, and if a therapy is started in hospital, we're committed to keeping that therapy'; however, the decision to institute therapy is often made without taking into account the GP's experience and long working knowledge of the patient.

Because it seems to be that we look after patients in general practice and then they go into this hospital - which is a bit like a spare parts body shop - and things happen to them, and they come back. It would be great if this system was more integrated. I mean, I know there's a move for patients to be discharged postoperatively a lot earlier for surgical conditions. And I think the success of that would really depend on an integrated community approach rather than just someone being sent out. ... general practice is not just a nebulous world, but we have a lot to offer, a lot of tacit knowledge that they can benefit from, and basically the patient [can benefit]. GP4

Following surgery two GPs said that they like to make goals with their patients to help them get back to their previous level of function (so long as it is realistic), and to instigate services that are necessary for the patient to achieve their best quality of life 'self-caring'. One of these 
participants noted however that it is 'often too late by the time I see them - they might come in three or four weeks after they've been discharged'.

All of the GPs said they were not privy to surgeons' postoperative care plans or preferences (including thromboprophylaxis). One GP described sometimes looking 'like a bit of a fool' when patients fronted up after a surgery with questions about the postoperative plan. Another GP expressed adamantly that it is 'important that we know when the patients are going in'. She suggested that one strategy to keep the GP involved would be for them to start the pre-anaesthetic paperwork in primary care; this way they could also update surgeons on any changes since their original referral letter.

While GPs on the whole stated that they were not familiar with thromboprophylaxis guidelines, they all agreed that being provided information and postoperative management advice by specialists 'would be very helpful'.

We just need clear, concise, accurate, straightforward, you know, information. I'm quite sure that one could deliver most of that on, on a single sheet, that's succinct and relevant, it doesn't need to have a load of junk attached to it. GP1

Another GP said guidelines imbued her with the 'confidence' to speak to patients about their options so that they can make an 'informed decision', because 'ultimately it is the patient's decision'.

\subsection{Discussion}

Significantly, all of the participants in this study agreed that the provision of thromboprophylaxis to arthroplasty patients is ultimately the operating surgeon's decision. Although all participating surgeons reported routinely prescribing thromboprophylaxis postoperatively, they differed significantly in their prescribing preferences. Many interrelated factors influenced these preferences; however, none more so than a surgeon's opinion: those who believed strongly in aspirin's safety and ability to prevent VTE were more likely to prefer it, and the same was true of anticoagulants.

Interestingly, a surgeon's opinion was greatly shaped by their training and collective experience, and not by contemporary guidelines. Surgeons described regularly debating postoperative thromboprophylaxis requirements amongst themselves, and subsequently, they were often aware of their colleagues' prescribing preferences and associated complication rates. This knowledge influenced participants' opinions about different pharmacological agents and hence, also ultimately influenced their prescribing preference.

Notably, not all postoperative complications had the same bearing on practice; both the severity and the type of complication affected its degree of influence. For example, serious isolated 
incidents such as the spinal bleed and fatal PE incidents significantly impacted participants' anticoagulant use thereafter. However, of the more commonly occurring less devastating postoperative complications (DVTs \& bleeding/wound ooze), only postoperative bleeding complications and infections were found to influence prescribing. This may be because DVTs were often described as being an accepted complication of surgery - 'if I look hard enough I will find a clot in every one of my knee replacements and every one of my hip replacement patients' whereas bleeding complications (and consequently infections) were generally perceived to be preventable complications, predominantly associated with anticoagulant use rather than the surgery itself. Remarkably, participants described bleeding and infection complications as disastrous and nightmarish; however, no such adjectives were used to describe the consequences of non-fatal postoperative VTE.

Despite the potential for postoperative complications to play a role in shaping surgeons' prescribing, the surgeons in this study acknowledged that they were probably unaware of the true incidence of these complications in their patients. This is a classic example wherein a practice gap reflects a system issue and not solely provider performance. ${ }^{178}$

The sentiment expressed by multiple surgeons that you can find literature to support any argument was particularly noteworthy, and indicates that study data does not influence a surgeon's opinion on the safety and efficacy of pharmacological agents to the same extent that personal reallife experiences do. In particular, studies that use asymptomatic VTE reduction as a marker for symptomatic VTE reduction were described as having little (if any) clinical relevance. Interestingly, participants in the study outlined in Chapter 6 described the inclusion of this type of data in the NHMRC Guideline as being one reason why the Guideline was deemed inappropriate:

'The guidelines are based on inappropriate surrogates for thromboembolism which significantly detracts from their scientific validity.'

'End points of research do NOT include fatal PE or post thrombotic syndrome, so relevance is questionable.'

Furthermore, surgeons in this study described industry sponsorship of a study as a factor that immediately biased them against accepting the study's findings as true. Similar sentiments were also described by surgeons who completed the survey outlined in Chapter $6 .^{206}$

Although surgeons differed in their thromboprophylaxis preferences, they all agreed on the importance of tailoring their decision to the individual patient. Some patient factors encouraged certain prescribing behaviours (positive factors); while others discouraged them (negative factors). For example, similar to the findings presented in Chapter 6, anticoagulant use was positively influenced by a previous history of VTE, and negatively impacted by a previous history of bleeding and, to some extent, advanced age. Heavy perioperative bleeding negatively impacted some surgeons' thromboprophylaxis use postoperatively; however, others regarded it as an accepted surgical risk that is routinely remedied with blood replacement. Ensuring patient 
satisfaction and compliance were described as factors that positively influenced some surgeons' antiplatelet use; however, whether this was a factor that influenced their practice, or was a means of justifying their practice is uncertain.

In addition to the patient factors listed above, the presence of unit protocols positively influenced prescribing of anticoagulants; this is particularly noteworthy when reflecting on the findings of Chapter 5. However, the sphere of a protocol's impact was generally limited to the hospital in which the protocol existed. This indicates that surgeons generally favour prescribing autonomously, a practice that was described as being particularly encouraged by the working environment and culture in Australia. It is possible however that this may change in the coming years as surgeons' professional autonomy is increasingly impacted negatively by the expansion of legal regulation in Australia. ${ }^{141}$

Unlike the findings reported by Molnar et al, medico-legal concerns were not found to be a dominating influence for participants. ${ }^{121}$ This may be because medico-legal exposure in Tasmania 'is not as bad' as it is in other states and territories, and the bulk of surgeons who participated in Molnar et al's study were from other states and territories.

Four barriers reported by Duff et al in the uptake of thromboprophylaxis guidelines were a lack of motivation to change, a lack of systems support, disputed evidence and a knowledge or awareness deficit; elements of these were also identified in this study. ${ }^{177}$ For example, although all surgeons expressed familiarity with at least one contemporary guideline, GPs generally did not. This lack of knowledge and familiarity naturally impairs their ability to contribute to postoperative care, even though they are ideally positioned to play a prominent role in preventing VTE after discharge. That GPs have chosen to step back (consciously or unconsciously) and defer to directives from hospital staff is of concern, as the communication between the hospital and community was frequently described as being poor and slow. Such communication delays would inevitably impact negatively on the speed and ability of GPs to provide primary care postdischarge, and in the end leaves the patient at a significant disadvantage.

A quick ready-reference protocol could assist greatly in overcoming both communication and knowledge obstacles. Rather than awaiting the formulation of a Tasmanian based protocol, another option would be to instigate a statewide strategy to increase the uptake of the NHMRC Guideline. One of the biggest barriers identified in this study (and the study outlined in Chapter 6) to the adoption of guidelines such as the NHMRC Guideline is the perception that they impose a 'one size fits all' approach to thromboprophylaxis by making 'carte blanche recommendations'. In reality, the NHMRC Guideline takes a comprehensive approach to VTE prophylaxis and unbeknown to most of the participants in this study, actually addresses the many concerns they expressed in the interviews:

'The Committee acknowledges the continuing debate on the clinical relevance of asymptomatic distal DVT as an indicator of the efficacy of VTE prophylaxis. Some experts have argued that 
guideline committees should consider evidence relating only to symptomatic VTE or to symptomatic PE. This Committee's decision to consider all thrombosis or embolism events as relevant outcomes was based on the fact that VTE encompasses a spectrum of disease, from asymptomatic distal DVT to fatal PE, and that most events are initially asymptomatic. Notwithstanding this, data on symptomatic DVT and PE were weighted more highly in the Committee's decision making process, and no recommendations were based on asymptomatic outcomes alone.

...The risk of VTE in hospitalised patients must be balanced against the actual and perceived risks of pharmacological thromboprophylaxis and patients' tolerance of pharmacological (especially injectable) or mechanical prophylaxis.

... By nature of its mechanism of action, pharmacological prophylaxis may increase the risk of surgical bleeding.

...As the evidence presented throughout this Guideline is mostly from randomised controlled trials, this may not be an accurate reflection of the incidence of bleeding outside the controlled trial context. .. An assessment of bleeding risk is an essential step in deciding on appropriate thromboprophylaxis for individual patients.

...In particular, surgeons may be understandably reluctant to expose patients to the risk of excessive intra- or post-operative bleeding and the subsequent complications, especially in procedures such as joint replacement where bleeding can lead to severe infections and a need to explant prostheses.

...It is essential to perform and record a VTE risk assessment in each patient before deciding whether or not to use preventive measures and on the most appropriate measures to use... The final decision to provide thromboprophylaxis is a clinical decision based on number and type of risk factors balanced against risk of bleeding.

... The recommendations are intended to encapsulate the available evidence on the prevention of VTE. However, they should only be followed subject to the judgement of clinicians caring for individual patients and patients' own preferences.

... Patient compliance is an important consideration in choice of thromboprophylactic agent. It is advised that this decision about the most appropriate type of thromboprophylaxis is made in consultation with the patient to increase acceptability and improve compliance. ${ }^{17}$

Significantly, only one participant was aware that the NHMRC Guideline presents data on both the benefits and harms of different prophylaxis options, or that it provides a step-by-step tool to assist doctors in making thromboprophylaxis a clinical decision that is individualised to each patient. This may be because the NHMRC Guideline is a multidisciplinary guideline which includes thromboprophylaxis recommendations for eighteen patient groups over the span of 160 pages. Most doctors do not have the time (or desire) to read such a large document in its entirety and will understandably move straight to skimming the one or two pages they deem relevant to their specialty. Potentially producing a separate one or two page summary document for surgeons that expresses all of the relevant points above, coupled with the Guideline's recommendations, 
may result in greater acceptance and adoption of the national guideline (see Appendix E for an example).

Many of the health professionals interviewed in this project were practising in Tasmania, which in itself has an interesting reality. It is an island state with a relatively small population. Being an island, 'you have a bit of an island mentality that we should cover for our little island'. With only three public hospitals in the state that offer major orthopaedic surgery, and only eighteen orthopaedic surgeons practising in the state, there is potential for implementing statewide changes relatively easily.

In Tasmania we have the chance to do things really well. We're small enough, we can get on - we can do things; whereas in larger populations it can be a bit more difficult. But that depends on leaderships [sic], and depends on having standards. And relies on people doing things well. GP1

A simple education strategy to inform relevant health professionals of the content and evidence base of the NHMRC Guideline and a strategy to improve two-way communication between hospital and community spheres (both to improve GP knowledge of each individual's postoperative surgical care plan and to improve surgeons' awareness of their postoperative complication rate) are two simple methods that could go a long way in reducing practice gaps in Tasmania; both should be relatively easy to implement in our island state.

Although there are many health professionals involved in the care of a patient from admission to discharge, the decision to prescribe thromboprophylaxis is ultimately the operating surgeon's to make. There were many factors that influenced surgeons' perception regarding the efficacy and safety of thromboprophylaxis agents, and therefore their prescribing preferences. Any strategies to optimise and streamline prescribing must address their concerns, or else the strategies risk complete failure. 


\section{The NeW ACCP GUIDELINE}

\section{Introduction}

The retrospective review discussed in Chapter 3 was undertaken in 2010, reviewing data from 2007 to 2009. After comparing local prescribing practices with the $7^{\text {th }}$ edition of the ACCP Guideline it was determined that only $5.0 \%$ of patients ( $8.0 \%$ of hips, $1.0 \%$ of knees) received recommended courses of thromboprophylaxis, predominantly due to a reluctance to prescribe anticoagulants following discharge. The audit of several hospitals in Australia (Chapter 4) indicated prescribing was considerably different to guideline recommendations. These findings suggested that there was a nationwide guideline-practice gap.

The studies outlined in Chapters 5, 6 and 7 identified many barriers to guideline adoption and thromboprophylaxis use; in particular, concerns over both bleeding complications, and the threat of guidelines being too prescriptive. Furthermore, $80.0 \%$ of surgeons who completed the survey in Chapter 6 were dissatisfied with the NHMRC Guideline because it does not include aspirin as a means of sole prophylaxis, recommends an apparently excessive long duration of prophylaxis and was perceived to rely heavily on pharmacological prophylaxis (which again was associated with surgeons' bleeding concerns). Significantly, the biggest reason why surgeons felt the Guideline was inappropriate was that they believed it was based on an inappropriate evidence base (Table 25). As the NHMRC recommendations are similar to the previous ACCP guidelines (Table 26), the findings of the study outlined in Chapter 6 could be extrapolated to indicate that the $7^{\text {th }}$ and $8^{\text {th }}$ edition ACCP guidelines were also deemed inappropriate. This is likely why prescribing differed considerably when compared to the ACCP $7^{\text {th }}$ and $8^{\text {th }}$ edition guidelines in Chapter 3 . 
Table 26. Comparing the NHMRC and ACCP guidelines.

Recommended agents are highlighted in blue with gradings noted where applicable (e.g. $A, 1 B$, see Appendix $A$ ).

\begin{tabular}{|c|c|c|c|}
\hline Agent & ACCP $7^{\text {th }}$ Ed. $^{108}$ & ACCP $8^{\text {th }}$ Ed. $^{164}$ & NHMRC $^{17}$ \\
\hline Aspirin & $x$ & $x$ & $x$ \\
\hline Dabigatran & & & B \\
\hline Fondaparinux & $1 \mathrm{~A}$ & $1 \mathrm{~A}$ & B \\
\hline LMWH & $1 \mathrm{~A}$ & $1 \mathrm{~A}$ & A \\
\hline Rivaroxaban & & & B \\
\hline VKA & $1 \mathrm{~A}$ & $1 \mathrm{~A}$ & $x$ \\
\hline Duration & $\begin{array}{c}\text { Min. } 10 \text { days, up to } 35 \\
\text { days }\end{array}$ & $\begin{array}{c}\text { Min. } 10 \text { days, up to } 35 \\
\text { days }\end{array}$ & Up to 35 days \\
\hline Mechanical & & & GCS, IPCD, or VFP, until fully mobile \\
\hline Aspirin & $x$ & $x$ & $x$ \\
\hline Dabigatran & & & B \\
\hline Fondaparinux & $1 \mathrm{~A}$ & $1 \mathrm{~A}$ & B \\
\hline LMWH & $1 \mathrm{~A}$ & $1 \mathrm{~A}$ & A \\
\hline Rivaroxaban & & & B \\
\hline VKA & $1 \mathrm{~A}$ & $1 \mathrm{~A}$ & $x / \checkmark$ \\
\hline Duration & Min. 10 days & $\begin{array}{c}\text { Min. } 10 \text { days, up to } 35 \\
\text { days }\end{array}$ & Up to 14 days \\
\hline Mechanical & $\underline{\mathrm{OR}} \operatorname{IPCD}(1 \mathrm{~A})$ & $\underline{\mathrm{OR}}$ IPCD (1A) & GCS, IPCD, or VFP, until fully mobile \\
\hline
\end{tabular}

NB: $x=$ recommendation against agent use; $x / \checkmark=$ only recommended under special conditions.

See Appendix A for more details on recommendation gradings.

In January 2012 the ACCP released the ninth edition of their thromboprophylaxis guideline. ${ }^{68}$ Unlike previous editions, each section of the Guideline was written by clinicians expert in methodology and interpretation of the evidence, rather than by world experts in the field of thromboprophylaxis. ${ }^{207}$ Furthermore, in contrast to previous editions, the new Guideline acknowledges the limitations of using asymptomatic DVT as a surrogate outcome, and incorporates strategies to estimate reductions in symptomatic DVT and PE with thromboprophylaxis. ${ }^{207}$ This has resulted in significant changes to the thromboprophylaxis recommendations (Table 27). 
Table 27. Changes in ACCP Guideline.

\begin{tabular}{|c|c|c|c|c|}
\hline \multirow[b]{2}{*}{ Topic } & \multicolumn{2}{|c|}{ ACCP $8^{\text {th }}$ Edition $^{164}$} & \multicolumn{2}{|c|}{ ACCP $9^{\text {th }}$ Edition ${ }^{68}$} \\
\hline & Recommendation & Grading & Recommendation & Grading \\
\hline Aspirin as a sole agent & $x$ & $1 \mathrm{~A}$ & $\checkmark$ & 1B \\
\hline $\begin{array}{l}\text { Mechanical } \\
\text { prophylaxis as sole } \\
\text { prophylaxis }\end{array}$ & $x$ & $1 \mathrm{~A}$ & $\checkmark$ & $1 \mathrm{C}$ \\
\hline $\begin{array}{l}\text { Duration of } \\
\text { prophylaxis for hip } \\
\text { patients }\end{array}$ & $10-35$ days & $1 \mathrm{~A}$ & $10-35$ days & $2 B$ \\
\hline
\end{tabular}

NB: $x=$ recommendation against; $\checkmark=$ recommendation for .

Grade 1 recommendations are strong and indicate that the benefits do or do not outweigh risks, burden, and costs. Grade 2 suggestions imply the individual patient values may lead to different choices. See Appendix A for more details

To identify how surgeons' prescribing compared to current recommendations, the original retrospective data presented in Chapters 3 and 4 were reanalysed against the new recommendations (Chapter 8). To determine if the changes to the recommendations were generally agreeable to the orthopaedic community a second online survey of surgeons was conducted (Chapter 9). 


\section{Chapter Eight: Guideline Practice Reanalysis}

\subsection{Aim}

To compare the prescribing presented data in Chapters 3 and 4 with the ACCP $9^{\text {th }}$ Ed. Guideline.

\subsection{Methods}

The data pertaining to pharmacological thromboprophylaxis prescribing presented in Chapters 3 and 4 were revisited and the findings compared to the ACCP $9^{\text {th }}$ Ed. Guideline.

\subsection{Results}

\subsubsection{RHH Retrospective Data (from Chapter 3)}

In total, $39.8 \%$ of knee arthroplasty patients and $22.3 \%$ of hip arthroplasty patients who underwent a hip and/or knee arthroplasty between June 2007 to 2009 received thromboprophylaxis consistent with the new ACCP recommendations (Figure 25). This equates to $31.5 \%$ overall.

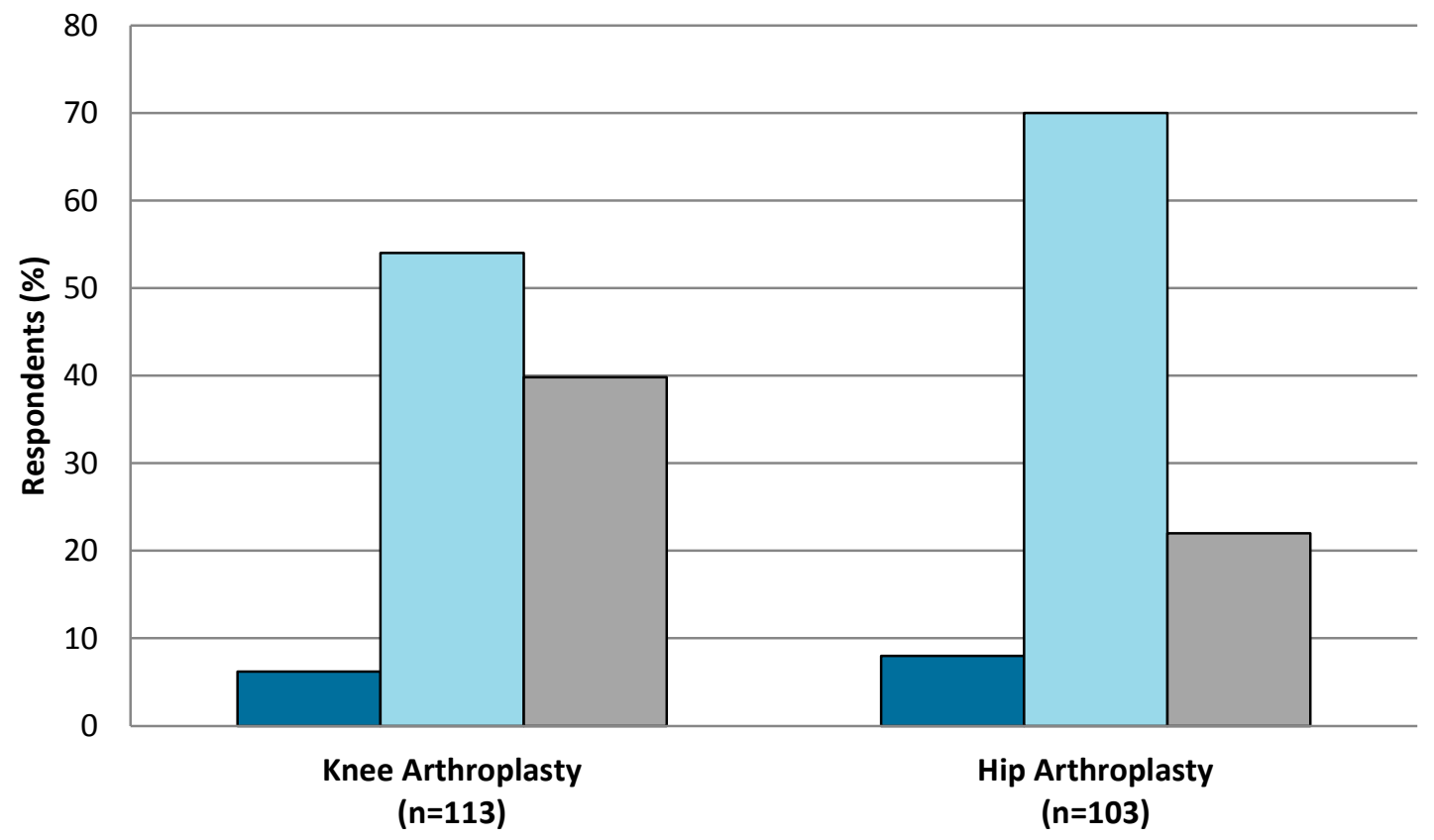

Figure 25. Comparison of RHH prescribing practices with the ACCP $9^{\text {th }}$ Ed. Guideline $(N=216)$

Appropriate agent only

Appropriate agent and dose only

Appropriate agent, dose and durations

NB: Excludes patients prescribed therapeutic anticoagulation for pre-existing medical conditions, and patients who experienced a bleed or VTE during their inpatient admission. 


\subsubsection{Collaborative Hospital Data (from Chapter 4)}

In total, $75.0 \%$ of knee arthroplasty patients and $72.7 \%$ of hip arthroplasty patients received thromboprophylaxis consistent with the new ACCP recommendations (Figure 26). This equates to $73.8 \%$ overall. This did not significantly change when the appropriateness of doses prescribed was not included in the analysis (Figure 27).

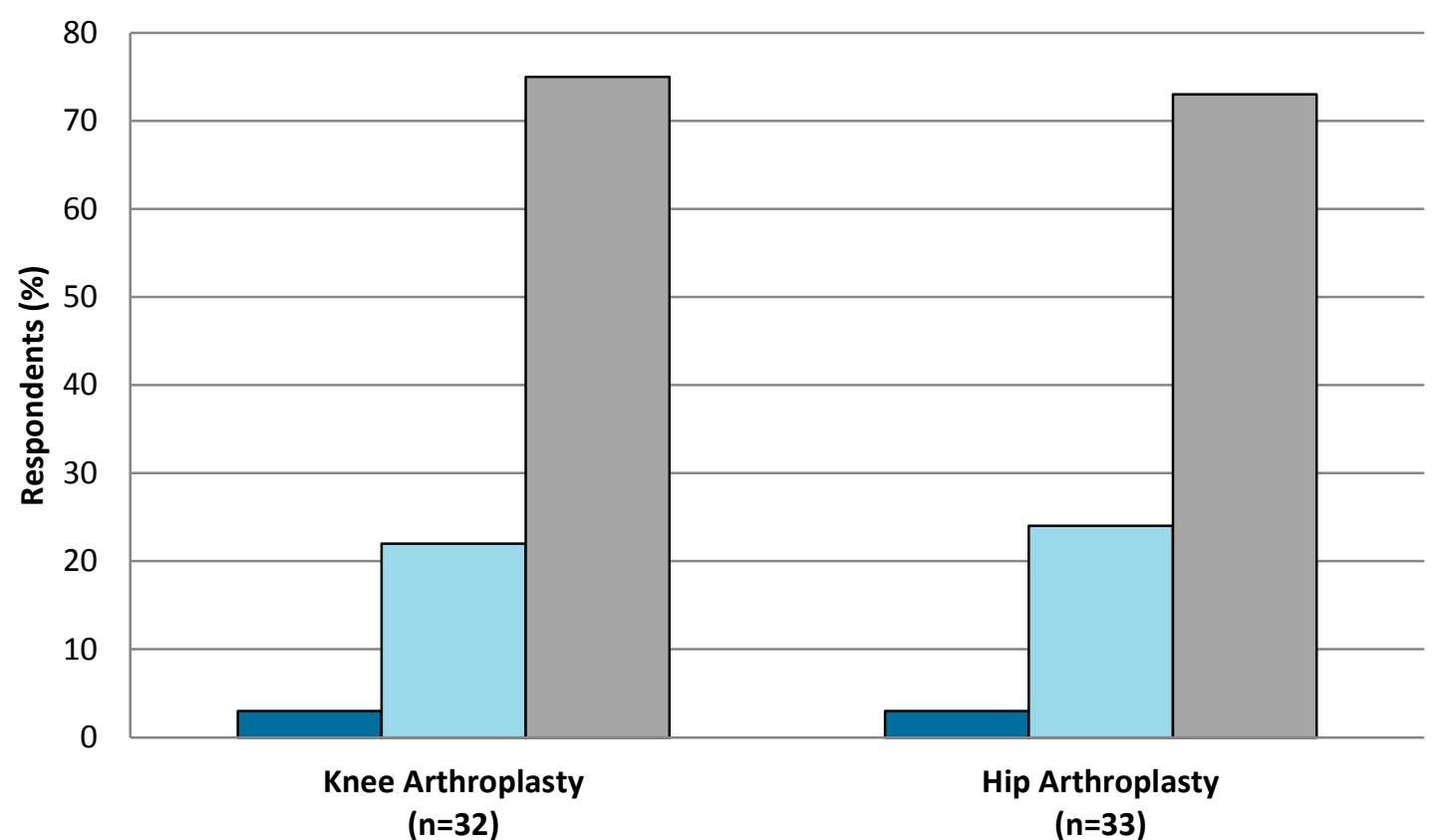

Figure 26. Comparison of multicentre prescribing practices with the ACCP $9^{\text {th }}$ Ed. Guideline $(N=65)$.
Appropriate agent only
Appropriate agent and dose only
Appropriate agent, dose and duration

NB: Excludes patients prescribed therapeutic anticoagulation for pre-existing medical conditions, and patients who experienced a postoperative complication during their inpatient admission.

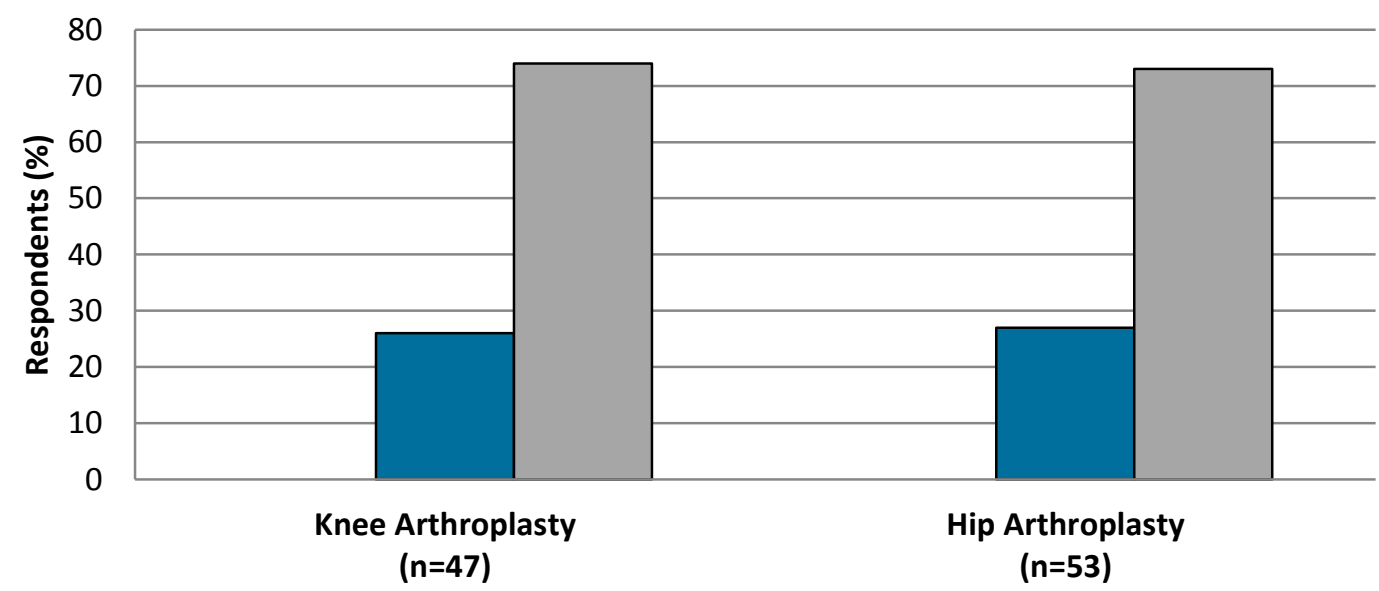

Figure 27. Comparison of multicentre prescribing practices with the ACCP $9^{\text {th }}$ Ed. Guideline $(N=100)$.

Appropriate agent only

Appropriate agent, dose and duration

NB: Excludes patients prescribed therapeutic anticoagulation for pre-existing medical conditions, and patients who experienced a postoperative complication during their inpatient admission. 


\subsection{Discussion}

The findings in this short reanalysis reveal a significantly greater compatibility between practice and the new ACCP Guideline. Whereas previously only $5.1 \%$ of patients on average undergoing a hip or knee arthroplasty at the RHH were prescribed complete courses of thromboprophylaxis according to the ACCP $7^{\text {th }}$ Ed. Guideline, almost $32 \%$ were prescribed complete courses according to the ACCP $9^{\text {th }}$ Ed. Guideline. Similarly, whereas only $53.8 \%$ of patients at the six hospitals surveyed in 2011 were prescribed NHMRC recommended thromboprophylaxis, 73.8\% were prescribed thromboprophylaxis as recommended in the ACCP $9^{\text {th }}$ Ed. Guideline. In both cases, the decrease in the guideline-practice gap was predominantly due to the adoption of aspirin as an appropriate option for pharmacological thromboprophylaxis in the ACCP $9^{\text {th }}$ Ed. Guideline.

This reanalysis suggests prescribing patterns in previous years were generally more aligned with contemporary recommendations than recommendations existent at the time of prescribing; i.e. that practice preceded guidelines. This occurrence is likely due to a combination of factors; however two interconnected ones stand prominent. The methodology used to produce the ACCP $9^{\text {th }}$ Ed. Guideline was reported to be more rigorous than previous editions, resulting in a guideline that is less based on opinion, and more on research data. ${ }^{207}$ Clear noncontroversial and evidence based recommendations are more likely to be employed than guidelines with recommendations that are unclear, controversial, or are based on opinion. ${ }^{20}$ This change in methodology may in part explain the increase in compatibility between practice and the updated ACCP Guideline.

Turner et al report that although there has been a significant rise in the number of CPG produced worldwide, few of these CPG meet quality criteria; 'the link between research and recommendations is an area of particular weakness' ${ }^{20}$ Guideline production is a lengthy and often time-consuming process, thereby negatively impacting on the ability of a professional body to make recommendations based on the most up to date evidence. This limitation is likely to be augmented for professional bodies such as the NHMRC and ACCP, as they produce multidisciplinary guidelines. As noted in Chapter 7, surgeons review and discuss the latest research amongst themselves. They are able to implement changes immediately, and this may be why the results of this reanalysis suggest that practice preceded the guidelines. This limitation in guideline formulation must be addressed in order to prevent guidelines lagging behind the evidence. 


\section{Chapter Nine: Surgeons AND THE ACCP 9Th Ed. Guideline}

\subsection{Introduction}

The data reanalysis outlined in Chapter 8 determined surgeons had been prescribing more in line with the ACCP $9^{\text {th }}$ Ed. Guideline than the ACCP $7^{\text {th }}$ Ed. Guideline for up to four years prior to its' release. This suggested that the changes made to the Guideline were agreeable to surgeons in Australia. A second national survey of surgeons was conducted in order to explore surgeons' opinions of the AACP $9^{\text {th }}$ Ed. Guideline to determine if this was the case, and to identify if there had been any change in surgeons' opinions since the previous survey conducted two years earlier.

The previous survey (outlined in Chapter 6) had been targeted at ASA members and had resulted in a small survey sample group. In an attempt to increase absolute numbers, and thus reliability and generalisability of the study findings, this survey was sent to all hip and knee orthopaedic surgeons identified in Australia (see 9.3 Methods for details). This different survey pool also allowed for comparison between ASA members and non-members to determine if there was any inherent difference in their opinions and preferences; and to determine if ASA membership had any influence on guideline familiarity and acceptability. Lastly, the larger sample size also allowed for more reliable comparisons between aspirin and anticoagulant users, thereby adding more data to the investigation into enablers and barriers to prescribing different pharmacological prophylaxis.

\subsection{Aim}

The aim of this study was three-fold:

- to explore Australian surgeons' opinion of the ACCP $9^{\text {th }}$ Ed. Guideline;

- to compare the opinions of ASA members and non-members;

- to determine if ASA members' opinions had changed since the previous survey, conducted two years earlier; and

- to identify any differentiating characteristics between aspirin and anticoagulant users.

\subsection{Methods}

The absolute number of respondents to the survey outlined in Chapter 6 was small (25 surgeons), thereby limiting the generalisability of the findings and increasing the risk of nonresponse bias. Consequently, a number of strategies were employed to increase the absolute number of responses to this survey, many consistent with those trialled in Chapter 5. These strategies included: 
- opening the survey to all surgeons in Australia, irrespective of whether they were ASA members or not,

- shortening the survey considerably from the template used in Chapter 6,

- making the survey available in both a soft and hard copy format,

- including a hard copy of the survey in every reminder letter so that surgeons could see how short and quick it was to complete,

- personally signing and including a personal 'thank you' note in each invitation and reminder letter,

- distributing the survey directly from the university, thereby excluding any potential bias which may have been introduced by the professional endorsement of the previous survey, and lastly,

- using a $3^{\text {rd }}$ generation Apple iPad draw (valued at AUD539) as an incentive to elicit participation.

\subsubsection{Participant Selection and Invitation}

The names and addresses of hip and knee surgeons were identified by searching through telephone listings and orthopaedic practice websites, and through general internet searches. An invitation to participate in the survey was sent to 485 surgeons in total. Some invitations were returned unopened because the surgeon was no longer at the said-practice. In these instances, follow-on practice details (where available) were identified and invitations (and follow-on reminders) were sent to the new practice.

\subsubsection{The Survey}

The survey was created with LimeSurvey and hosted on a University of Tasmania server. It contained a mixture of quantitative and qualitative questions that were divided into three sections covering: demographics and professional practising information; prophylaxis effectiveness and preferences; and lastly questions concerning familiarity with and opinion of contemporary guidelines (Appendix F). The quantitative styled questions were similar to those used in the study outlined in Chapter 6, however the qualitative open ended questions were focused on the ACCP $9^{\text {th }}$ Ed. Guideline instead of the NHMRC Guideline. ${ }^{68}$ To ensure all participants had equal opportunity to provide an informed opinion on the ACCP Guideline the survey included a summary of its recommendations. Participants were asked to indicate whether they were ASA members or not in order to allow comparison of responses between this and the previous surgeon survey (and between ASA and non-ASA members' responses).

The qualitative styled section included a space for participants to share their opinion on appropriate and inappropriate aspects of the ACCP $9^{\text {th }}$ Ed. Guideline, as well as general comments 
on thromboprophylaxis. This approach allowed exploration of their opinions in a way that checkbox styled questions could not achieve; and minimised responses being influenced by the researchers' presumptions.

The survey was open for participants to access for eight weeks in early 2012; during this time two reminder letters were sent encouraging surgeons to participate.

\subsubsection{Data Analysis and Statistics}

All data was entered into an Access database (Microsoft ${ }^{\circledR}$ Access 2010) and then transferred into SPSS 19.0 and NVivo 10 (QSR International® Doncaster, Victoria, Australia) for analysis of the quantitative and qualitative data respectively. The data was analysed only after the data collection phase had been completed. The methods of data analysis are described below for each style.

\subsubsection{Quantitative Data}

The differences between groups were tested using the t-test for independence for continuous data, and the chi square test for categorical variables. Pearson's rank correlation coefficients were calculated for measuring correlations. Continuous variables were summarized as means with standard deviation. A univariate analysis, followed by a multivariate analysis of variables, was performed to assess the contribution of surgeon variables on their prescribing preferences and opinions. Only $\mathrm{p} \leq 0.05$ values were considered statistically significant.

\subsubsection{Qualitative Data}

The transcription of comments made in the survey into the Access database was combined with reflective review. The data was then transferred into NVivo, and each surgeon's comments were separated into different documents identified by their survey ID number. Each document was visited sequentially and open coding was performed to group comments into thematic categories. Documents were revisited when new nodes were introduced that resonated with documents already coded.

\subsection{Quantitative Results}

\subsubsection{Response Rate}

In total, two hundred and fifty-eight surgeons responded to the invitation letters, however some responses could not be included in the analysis (Table 28). The final response rate was 49.2\% (221, $\mathrm{N}=449)$. Most completed responses were submitted as hard copies; however 9 respondents $(4.1 \%)$ completed the survey online. 
Table 28. Break down of responses $(\mathrm{N}=258)$.

\begin{tabular}{|l|l|l|}
\hline & $\mathbf{n}$ & $\mathbf{\%}$ \\
\hline Uncertain if respondent conducted hip or knee arthroplasty & 4 & 1.6 \\
\hline Respondent did not conduct hip or knee arthroplasty & 7 & 2.7 \\
\hline $\begin{array}{l}\text { Invitation letter returned because surgeon left country, no longer practising, } \\
\text { terminally ill or deceased }\end{array}$ & 25 & 9.6 \\
\hline $\begin{array}{l}\text { Responded } 8 \text { months after the close of the survey } \\
\text { Responded with completed survey }\end{array}$ & 1 & 0.4 \\
\hline
\end{tabular}

\subsubsection{Demographics}

Most respondents were males $(217,98.2 \%, \mathrm{~N}=220)$, with an average of 17.8 years practising as orthopaedic surgeons (SD 9.0). Most participants practised in Queensland, New South Wales and Victoria (Table 29) and predominantly in the private sector $(155,70.7 \%)$, followed by both sectors equally $(55,25.1 \%, \mathrm{~N}=220)$.

Table 29. Break up of responses from each state and territory $(\mathrm{N}=221)$.

\begin{tabular}{|l|l|l|}
\hline & $\mathbf{n}$ & Overall \% \\
\hline Australian Capital Territory & 1 & 0.5 \\
\hline New South Wales & 59 & 26.7 \\
\hline Northern Territory & 3 & 1.4 \\
\hline Queensland & 49 & 22.2 \\
\hline South Australia & 21 & 9.5 \\
\hline Tasmania & 12 & 5.4 \\
\hline Victoria & 61 & 27.6 \\
\hline Western Australia & 15 & 6.8 \\
\hline
\end{tabular}

Participants conducted an average of 14.6 arthroplasties each month; with a slight preponderance for knee arthroplasties (6.0 hips vs. 8.9 knees, SD 14.6), which is reflective of the national trend. There was no correlation between the number of years practising and the number of arthroplasties participants conducted monthly.

Forty-eight participants $(23.0 \%, \mathrm{n}=209)$ identified themselves as ASA members. There was no significant demographic difference between members and non-members, however ASA members reported conducting significantly more arthroplasties each month (21 vs. $13, \mathrm{p}<0.001)$.

\subsubsection{Prescribing Practices}

\subsubsection{Agent Preference}

Most surgeons $(213,95.9 \%)$ reported routinely prescribing pharmacological prophylaxis for their patients following surgery, and most (210) shared their preferred prescribing regimen. The regimens described included the following: 
- $\quad$ LMWH

- $\mathrm{LMWH} /$ rivaroxaban

- Aspirin if one knee, LMWH if two knees

- Apixaban

- Rivaroxaban

- Warfarin
- $\quad$ LMWH during inpatient stay, rivaroxaban at home

- Unfractionated heparin during inpatient stay, LMWH at home

- $\quad$ LMWH during inpatient stay, apixaban at home

- LMWH during the inpatient stay, aspirin at home

- Aspirin if low risk, LMWH if high risk

- Aspirin if low risk, rivaroxaban if high risk

Anticoagulants were the most popular choice $(154,75.1 \%)$, followed by a combination of an anticoagulant + aspirin $(28,13.7 \%$, Table 30$)$. LMWH was the most popular anticoagulant; however as noted above, a range of anticoagulants were regularly used by respondents.

Table 30. Primary pharmacological preferences (\%) between ASA and non-ASA members $\quad(\mathrm{N}=205)$.

\begin{tabular}{|l|c|c|c|c|}
\hline & Overall & ASA member & Non-ASA member & $p$ \\
\hline Aspirin & 11.2 & 17.0 & 9.5 & 0.12 \\
\hline Anticoagulant & 75.1 & 63.8 & 78.5 & 0.5 \\
\hline Aspirin + anticoagulant & 13.7 & 19.2 & 12.0 & \\
\hline
\end{tabular}

NB: Eleven people did not indicate if they were an ASA member or not, and a further five people did not indicate their prescribing preference.

There was no statistically significant difference in agent choice between ASA members and non-members; however there was a trend towards more members favouring aspirin $( \pm$ an anticoagulant) compared to non-members. There was no significant correlation between agent preference and surgeon demographics.

\subsubsection{Duration Preference}

Most participants preferred continuing pharmacological prophylaxis post-discharge; and knee arthroplasty patients were more likely to receive shorter courses of thromboprophylaxis than hip arthroplasty patients $(\mathrm{p}<0.001$, Table 31$)$.

Table 31. Preferred duration of thromboprophylaxis (\%).

\begin{tabular}{|l|c|c|}
\hline & Hip Arthroplasty $(n=176)$ & Knee Arthroplasty $(n=190)$ \\
\hline 3-4 days & 1.7 & 1.6 \\
\hline $5-7$ days & 8.5 & 10.5 \\
\hline $8-14$ days & 9.7 & 32.1 \\
\hline $15-21$ days & 10.2 & 18.4 \\
\hline $22-28$ days & 21.0 & 11.1 \\
\hline $29-35$ days & 25.6 & 7.9 \\
\hline $36-42$ days & 21.6 & 16.8 \\
\hline 90 days & 1.7 & 1.6 \\
\hline Median & 28.0 (SD 14.1) & 15.0 (SD 14.7) \\
\hline number of days & & \\
\hline
\end{tabular}


Surgeons who preferred anticoagulants prescribed significantly shorter courses of thromboprophylaxis than surgeons who preferred aspirin \pm an anticoagulant (-14 days for hip arthroplasties and -21 days for knee arthroplasties, $\mathrm{p}<0.05)$. There was no correlation between duration preference and ASA membership or surgeon demographics.

\subsubsection{Thromboprophylaxis Efficacy}

Almost one third of surgeons $(29.1 \%)$ indicated that they did not believe pharmacological prophylaxis prevented fatal PE, $44.1 \%$ indicated it may potentially reduce fatal PE, and $26.8 \%$ responded that it definitely decreased fatal PE. There was a trend towards non-members being more confident in the ability of pharmacological agents to prevent fatal PE than ASA members; however this did not reach statistical significance (Table 32).

Table 32. Opinions on efficacy of pharmacological prophylaxis (\%, N=208).

\begin{tabular}{|l|c|c|c|}
\hline Pharmacological agents... & $\begin{array}{c}\text { ASA member } \\
(n=48)\end{array}$ & $\begin{array}{c}\text { Non-ASA member } \\
(n=160)\end{array}$ & $\mathbf{p}$ \\
\hline Do not decrease fatal PE & 27.1 & 29.4 & 0.08 \\
\hline Potentially decrease fatal PE & 56.2 & 40.0 & 30.6 \\
\hline Definitely decrease fatal PE & 16.7 & \\
\hline
\end{tabular}

\subsubsection{Factors that Influence Thromboprophylaxis Prescribing}

Previous VTE, prolonged preoperative immobility and morbid obesity were the top three factors surgeons indicated would prompt them to prescribe pharmacological prophylaxis following surgery (Table 33). The only statistically significant difference between ASA members and non-members was that ASA members were more likely to select prolonged surgery as a factor that would prompt them to prescribe pharmacological agents ( $55.1 \%$ vs. $36.1 \%$ p $<0.05)$. In addition to the factors laid out in the original question, 6 surgeons (2.7\%) indicated malignancy and/or family history of VTE would also prompt them to prescribe prophylaxis. 
Table 33. Patient factors that prompt surgeons to prescribe pharmacological prophylaxis $(n=170)$.

\begin{tabular}{|l|l|}
\hline Previous VTE & $\%$ \\
\hline Prolonged preoperative immobility & 96.5 \\
\hline Morbid obesity (BMI >40) & 78.2 \\
\hline HRT & 75.3 \\
\hline Previous thrombophlebitis & 65.3 \\
\hline Moderate obesity (BMI >30) & 57.1 \\
\hline Current smoker & 54.7 \\
\hline Surgery > 2 hours & 53.5 \\
\hline Previous vein surgery & 50.6 \\
\hline Age $\geq 70$ years & 40.6 \\
\hline Age $<70$ years & 35.9 \\
\hline General anaesthesia & 32.9 \\
\hline Preoperative Infection & 30.0 \\
\hline None of these factors & 17.6 \\
\hline None of the above (I routinely prescribe chemoprophylaxis for all of my patients) & 17.1 \\
\hline
\end{tabular}

The top two factors reported to limit participants' prescribing of pharmacological prophylaxis for arthroplasty patients were the conviction that it increases the risk of infection at the operation site, and the belief that patients would not be compliant (Table 34). Furthermore, bleeding risk was a concern for almost half of the respondents (44.3\%), particularly during the inpatient period. Aspirin users were more likely be concerned with bleeding complications than anticoagulant users $(88.0 \%$ vs. $37.7 \%, \mathrm{p}<0.001)$.

Table 34. Factors that limit pharmacological prophylaxis use $(n=221)$.

\begin{tabular}{|l|l|l|}
\hline & Inpatient (\%) & Discharge (\%) \\
\hline It increases the risk of infection at the operation site & 40.3 & 22.2 \\
\hline It is not safe as it causes too much bleeding & 39.8 & 22.2 \\
\hline It is not superior to mechanical methods & 24.0 & 13.6 \\
\hline It is too expensive & 1.8 & 4.1 \\
\hline It is too inconvenient & 4.1 & 18.1 \\
\hline Patients will not be compliant & 2.3 & 17.2 \\
\hline Poor continuation of care from hospital to community & 3.2 & 9.0 \\
\hline Study evidence isn't applicable to real-world populations & 14.5 & 15.4 \\
\hline The risks of VTE are low & 11.3 & 14.0 \\
\hline None of the above & 28.1 & 23.1 \\
\hline
\end{tabular}

A further $18.1 \%$ of participants felt that the inconvenience associated with thromboprophylaxis limited its prescribing at discharge. Notably, these participants prescribed significantly shorter courses of prophylaxis for their patients on average. This reached statistical 
significance following knee arthroplasty (17 days vs. 23 days, $\mathrm{p}=0.04)$. There were no significant differences between any of the ASA members' and non-members' responses.

\subsubsection{Familiarity with Contemporary Guidelines}

Between 169 and 191 surgeons answered different parts of the question regarding contemporary guideline familiarity. Those participants who did respond indicated greatest familiarity with the ASA, AAOS and NHMRC guidelines (Figure 28).

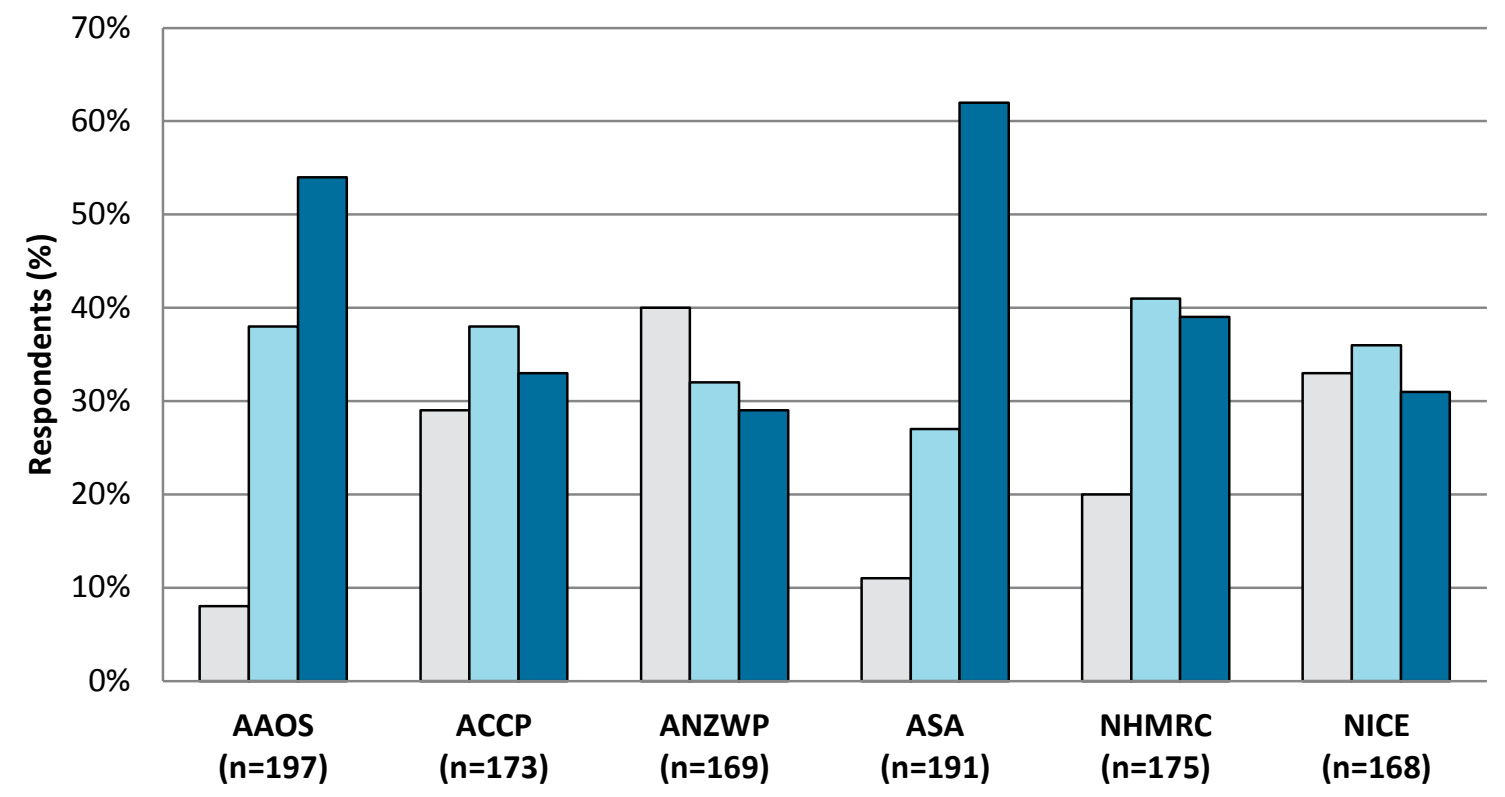

Figure 28. Surgeon familiarity with contemporary guidelines.

$\square$ I have not come across it before $\quad \square$ I have heard of it in passing $\square$ I am very familiar with it

ASA members were significantly more likely to be familiar with the ASA Guideline than nonmembers $(92.8 \%$ vs. $53.6 \%$, p $<0.001)$. Furthermore, there was a trend towards those who had practised longer as orthopaedic surgeons being more familiar with the contemporary guidelines. This finding reached statistical significance with the ANZWP, AAOS and ASA guidelines; surgeons who were very familiar with these guidelines had been practising for 5 to 8 years longer than surgeons who had only heard of them in passing $(\mathrm{p}<0.05)$.

\subsection{Qualitative Results}

This section included three open ended questions asking surgeons what they thought was appropriate and inappropriate about the ACCP $9^{\text {th }}$ Ed. Guideline, with space for further comments on thromboprophylaxis in general. Many comments directly addressed recommendations in the Guideline, however many respondents also commented generally on thromboprophylaxis and postoperative VTE prevention. These reflections are all included in the analysis below. 


\subsubsection{General Response}

One hundred and sixty nine surgeons (76.5\%) completed this section. Responses varied greatly from general 'I agree' statements to comments on specific recommendations that participants felt were appropriate and/or inappropriate, as well as suggestions on how to improve the Guideline. Forty-eight surgeons (28.4\%) generally agreed with the Guideline, however only two proclaimed to follow it. Another surgeon said he was considering aligning his practice with the Guideline after reviewing the summary of recommendations in the survey.

Surgeons who preferred prescribing an anticoagulant \pm aspirin were significantly more likely to agree with the Guideline than surgeons who preferred to routinely prescribe aspirin only (32.2\% vs. $0 \%, \mathrm{p}=0.003)$. There was no other significant difference between surgeons who agreed with the recommendations and those who did not, including demographics, reported familiarity with the ACCP Guideline and ASA membership.

\subsubsection{Anything is Better than Nothing}

The ACCP $9^{\text {th }}$ Ed. Guideline recommends the use of some form of pharmacological prophylaxis over nothing. Fourteen (8.3\%) surgeons highlighted this as an appropriate recommendation.

The use of something rather than nothing is appropriate. S103

All patients should receive prophylaxis unless contraindicated. S148

VTE prophylaxis probably saves lives and decreases morbidity, and [its] advantages outweigh disadvantages. $S 214$

Conversely, eight participants (4.7\%) questioned the validity of recommending pharmacological prophylaxis at all, saying it lacks efficacy and is associated with an increased risk of postoperative complications.

[There is] no diff[erence] in fatal PE rate with chemoprophylaxis. S22

There is no definitive evidence that chemoprophylaxis decreases the risk of PE. S225

Any of the above (except mechanical) all result in weeping wounds and worrisome low grade fevers at various times. $S 2$

I don't think any thromboprophylaxis has shown a definite decrease in fatal PE. I think we are over treating a huge number of patients with minor below knee thrombus. S39

These comments were in agreement with their responses earlier on in the survey with five having answered that pharmacological prophylaxis is not effective at all in preventing VTE, and the other three unsure as to its effectiveness. Despite this lack of confidence in its efficacy, all eight surgeons prescribed prophylaxis following surgery, most (62.5\%) choosing a sole anticoagulant as their preferred agent of choice. 


\subsubsection{Pharmacological Prophylaxis}

The new Guideline recommends the use of any one of the following prophylactic agents: LMWH; fondaparinux; dabigatran, apixaban, rivaroxaban; low dose unfractionated heparin; adjusted dose vitamin $\mathrm{K}$ antagonist; aspirin (all Grade 1B). LMWH is recommended in preference to the other agents (Grade 2C/2B).

This recommendation was received with mixed reactions (Table 35). There was no association between a participant's opinion and their demographics.

Table 35. Appropriateness of agent recommendations ( $\mathrm{N}=169)$.

\begin{tabular}{|l|l|l|}
\hline & $\mathbf{n}$ & $\%$ \\
\hline Aspirin is appropriate & 22 & 13.0 \\
\hline Aspirin is inappropriate & 8 & 4.7 \\
\hline LMWH is appropriate & 27 & 16.0 \\
\hline LMWH is inappropriate & 5 & 3.0 \\
\hline New Oral Agents are appropriate & 11 & 6.5 \\
\hline New Oral Agents are inappropriate & 7 & 4.1 \\
\hline Warfarin is appropriate & 6 & 3.6 \\
\hline Warfarin is inappropriate & 2 & 1.2 \\
\hline
\end{tabular}

\subsubsection{Aspirin}

Surgeons who commented positively on the recommendation to give aspirin as a sole agent were significantly more likely to prescribe it in practice ( $7 / 17$ vs. $8 / 127,41.2 \%$ vs. $6.3 \%$, p $<0.001)$. In contrast, only eight surgeons (4.7\%) questioned the recommendation, all of whom preferred an anticoagulant as their sole therapy.

Inappropriate: using aspirin as a viable VTE prophylaxis! No evidence. S59

Aspirin is clearly ineffective, how does it get grouped with the others? S214

One surgeon stated that he didn't use aspirin to prevent VTE, but 'mainly for decreased morbidity and mortality'.

\subsubsection{Anticoagulants}

Twenty six surgeons (15.4\%) agreed anticoagulants are appropriate choices for prophylaxis, most of whom (92.3\%) preferred to prescribe an anticoagulant \pm aspirin following surgery. Surgeons who agreed with the recommendation to use injectable anticoagulants following arthroplasty did not elaborate why, simply stating the recommendation was appropriate.

Appropriate: LMWH. 560 
Conversely, surgeons who supported the recommendation to use oral anticoagulant agents expounded further, describing them as 'preferable' because they are 'easier to administer in community, with equivalent efficacy' to injectable anticoagulants.

Oral agents are much more appropriate as injected compliance after discharge is v[ery] poor. We live in the real world. $S 92$

I am not convinced of [the] need to use injectables post-discharge - I don't feel they achieve much and are a little difficult for the patient - oral agents would be better when freely available. S175

Additionally, the regular monitoring required with warfarin use was described as an advantage.

Warfarin is monitored. Therefore, better control. If DVT [is] noted, the patient is already on the correct medication. As the only surgeon in a large hospital (15 active ortho[paedic] surgeons) using warfarin, the nursing staff [have] noted my complications are the lowest by far. S151

Surgeons who commented against the use of oral agents did so because of bleeding concerns. Rivaroxaban is new and probably dangerous. Our physicians have had TWO intrathecal bleeds from this protocol and find it is dangerous. S106

Inappropriate: recommendations for apixaban/dabigatran/rivaroxaban because of bleeding risk. $S 121$

The problem with warfarin is with bleeding, especially with knee replacement surgery, which sometimes by quite major. $S 119$

Side effects, risks i.e. mortality from warfarin > risk fatal PE. S174

There was no significant association between preferred agent and bleeding concerns.

\subsubsection{Low Molecular Weight Heparin in Preference to other Agents}

As noted earlier, the ACCP Guideline gives preference to LMWH over other agents. Eleven surgeons $(6.5 \%)$ disagreed with this recommendation, with only one surgeon explaining why:

No evidence to say this (2nd paragraph: we suggest the use of LMWH...). S36

Other participants did not outright disagree with the preference given to LMWH, however did comment that 'compliance with injections [is] difficult' and 'bad', that patients 'find it inconvenient' and that 'most won't self-inject'.

\subsubsection{Mechanical Thromboprophylaxis}

The Guideline recommends mechanical prophylaxis in the form of IPCD only. Four surgeons (2.4\%) commented that it 'should include foot pumps as an alternative to IPCD'. Another four commented against the use of IPCD altogether, stating that 'IPCD have NOT been shown to decrease PE' and are 'impractical for hip and knee replacements'. A further four surgeons commented that IPCD can irritate patients.

IPCD 10 to 14 days - impractical, not appropriate once patients are mobile. S29

IPCD impractical for prolonged period 10 to 14 days. I use first 48 hours when in bed. S57 
Calf compression devices frequently irritate patients and keep them bed/chairbound. (I use them [on] all patients hip/knee). S107

The Guideline recommends an IPCD in two scenarios: as a sole agent in patients with increased bleeding risk, and in combination with pharmacological means in all other patients. Thirty-seven surgeons (21.9\%) commented that recommending mechanical prophylaxis in either setting is appropriate. These comments are explored in more detail in the following two sections. There was no significant association between surgeon demographics, and opinion on mechanical prophylaxis; however a greater proportion of aspirin users commented positively on their use $(47.0 \%$ vs. $17.3 \%, \mathrm{p}=0.03)$

\subsubsection{Mechanical Thromboprophylaxis as a Sole Agent}

Six surgeons (3.5\%) specifically agreed with the recommendation to use mechanical thromboprophylaxis as a sole agent in patients with increased risk of bleeding, however one did note that 'patients at increased bleeding risk [are] not defined' and 'cannot [be] predict[ed]'. Most of these surgeons $(75.0 \%, \mathrm{~N}=4)$ reported prescribing aspirin as a sole agent postoperatively.

Five surgeons $(2.9 \%)$ commented that mechanical thromboprophylaxis as a sole agent was inappropriate. Most (80.0\%) of these surgeons reported prescribing anticoagulants as sole agents postoperatively, however often preferred to use a combination of methods.

I will use chemoprophylaxis in patients with increased bleeding risk. I can contain bleeding at surgery; I can't retrieve a fatal PE. I prefer combined mechanical, chemical and physiological (hydration \& movement) [prophylaxis]. S198

\subsubsection{Combining Mechanical and Pharmacological Prophylaxis}

Twenty four surgeons (14.2\%) specifically commented that the recommendation to use a combination of mechanical and pharmacological thromboprophylaxis is appropriate.

I agree with the first paragraph (and adding IPCD to chemoprophylaxis). S155

Appropriate: combining chemical prophylaxis with mechanical prophylaxis. S59

Only one surgeon questioned the evidence to support the recommendation.

I am unaware of evidence which state[s] that IPCD in addition to LMWH is of any benefit. S60

\subsubsection{No Thromboprophylaxis}

As an alternative to IPCD, the Guideline also recommends using no prophylaxis in patients at increased bleeding risk. The general reaction was to this mixed: four surgeons agreed, and four disagreed.

Appropriate: no prophylaxis in patients at increased risk of bleeding. S51

No prophylaxis advice, in the context, seems inappropriate. $S 168$ 
All eight surgeons reported prescribing anticoagulants as sole agents following hip and knee arthroplasty.

\subsubsection{Duration}

The Guideline recommends using pharmacological and mechanical thromboprophylaxis for a minimum of 10 to 14 days, and up to 35 days postoperatively. Participants commented that this range 'makes little sense' and leaves the 'length of time uncertain'.

'for up to 35 days' <-- give a better answer. S14

Specific comments on extended-duration mechanical and pharmacological prophylaxis are explored in the succeeding sections below.

\subsubsection{Duration of Mechanical Prophylaxis}

There were no positive comments about the recommendations to use IPCD for an extended period of time. Furthermore several surgeons $(6,3.5 \%)$ questioned the practicality and value of the recommendation.

How many patients have IPCD available to them after DC [discharge]? S33

Most patients following major joint arthroplasty are home by 7 to 10 days post op. Compression devices therefore only available as inpatient. 568

Extend IPCD only until patient mobile. S146

One day mechanical will be enough. 545

\subsubsection{Duration of Pharmacological Prophylaxis}

Opinions on the suitability of extended-duration pharmacological prophylaxis were significantly more varied. Six surgeons (3.5\%) commented positively on the recommendation, most of whom $(83.3 \%)$ preferred to prescribe anticoagulants postoperatively.

It is better to treat all patients as a potential risk and therefore [use] extended prophylaxis. S209

Three surgeons (1.8\%) commented that the minimum duration recommended was too short.

Length of chemoprophylaxis too short. Should be at least 4 weeks. S176

Conversely, thirty-one surgeons $(18.3 \%)$ commented that extended-duration therapy was inappropriately long and many questioned the motive and evidence to support the recommendation.

How good is [the] evidence for 35 days? S214

I don't think there is enough literature to support 35 days prophylaxis. I am aware of so called

2nd peak. S39

5 weeks of so called prophylaxis is too long. ... My view is that prophylaxis is appropriate for 2 to

3 weeks. $S 49$

Extended LMWH - good evidence till discharge date. No cost benefit analysis beyond this. S146

Inappropriate: extending thromboprophylaxis for 35 days. Seems like a drug pusher. S65 
There is no point continuing chemoprophylaxis if routine ultrasound is normal post op in a mobile patient. $S 188$

Thromboprophylaxis for 35 days is very excessive - only appropriate for very high risk patients (e.g. previous DVT/PE, known thrombophilia). S199

These surgeons generally prescribed shorter courses of thromboprophylaxis compared to surgeons who did not comment negatively on extended-duration therapy (average of 18 days vs. 29 days for hip arthroplasty patients, 14 days vs. 23 days for knee arthroplasty patients, $p<0.001$ ).

Other surgeons did not necessarily disagree with the recommendation to use prolonged thromboprophylaxis, however they commented that there are 'compliance' and convenience issues with using injectable agents at home.

Most of my arthroplasty patients leave hospital at day 3 or 4 post op, most won't self-inject. The use of district nurses for injections or INR tests is a considerable work load and expense. S176 Subcutaneous injections out of hospital - p[atien]t compliance is bad, except for patients I can convince that have high risk for DVT + PE. S10

\subsubsection{Other Comments}

In addition to the above, many surgeons commented more generally on the Guideline itself, as well as postoperative complications that had impacted on their prescribing over the years. These comments are summarised in the proceeding sections, 9.5.7.1 General Comments on the Guideline and 9.5.7.2 General Comments on Postoperative Complications, respectively.

\subsubsection{General Comments on the Guideline}

\subsection{The Evidence Base}

Many surgeons questioned whether there was any evidence to support the Guideline's recommendations.

These are based on low level evidence. S188

... they are vague, which reflects the scarcity of good clinical research in this area. S158 It is expert opinion, not based on scientific studies in orthopaedics. S70

There is no hard evidence there - just use something. S215

I am not aware of any evidence that thromboprophylaxis changes mortality from PE. S33

No evidence that any agent reduces P.E. S3

There is no definitive evidence that chemoprophylaxis decreases the risk of PE. S225

In summary, evidence is inconclusive... S214

Evidence for efficacy is inconclusive. S212

There is no convincing evidence for a lowering of mortality. S211

Lacks evidence (NO EVIDENCE) for the efficacy to prevent fatal PE. S116 
Some accused the Guideline of being 'largely driven by pharmaceutical companies, NOT evidence', noting 'there is a lot of pressure from the drug companies to use their drug'. They further alleged that industry supported studies biased the literature.

The late complications of antithrombotic usage have been greatly UNDER estimated in literature driven by pharmaceutical companies. S13

The outcome/recommendation is weighted towards chemoprophylaxis which relies heavily upon sponsored work in which there are possible conflicts of interest. S142

In addition to being described as 'inconclusive', the evidence to support recommendations was described as being out-dated and swayed by inappropriate surrogates for symptomatic VTE.

All studies use historical rates of DVT/PE and bleeds, and DVT as a marker for fatal PE. S131

Data on which study is based is out-dated, surgical protocols have evolved. S3O

35 days [is] too long - driven by old papers and pharmaceutical companies. S46

My biggest issue is in the literature, the use of symptomatic and asymptomatic VTE - THIS perpetuates bias. $S 144$

Data is not placebo controlled - therefore study in total is compromised. S3O

Overemphasised [level] I and II evidence. Ignore existing studies including Austria/Norway + Kaiser group. $S 41$

Extrapolations of VTE evidence to PE are of dubious scientific validity. S214

Duration recommendations are limited by government cost cutting \& doubtful scientific evidence. $S 193$

Furthermore, participants alleged that the Guideline was 'defensive more than anything scientific'.

It is driven more by medico-legal concerns than by evidence of effectiveness. S94

We are driven by medico-legal fears, NOT patient outcome. S21

It probably makes little or no different to PE risk, but it is a MEDICO-LEGAL requirement. S4

Several surgeons described feeling pushed into prescribing 'thromboprophylaxis as a routine' 'for medical insurance reasons', 'irrespective of what surgeons think'. They noted that this was 'dangerous' and projected that 'inappropriate prescribing' and 'overprescribing' 'due to poorly formulated and overly prescriptive recommendations' would eventually cause problems.

I feel blackmailed into using Clexane [enoxaparin] because of QHealth policies. S219

We will soon be dealing with litigation due to USE of these agents. S13

Amongst all of the concerns about the strength of the Guideline's evidence base, some surgeons conceded that it is difficult to produce and/or find good methodologically sound trials.

The amount of data required for statistical significant level 1 evidence is not obtainable. S215

No study will ever approach reliability with PTE because sample size required ideally for one surgeons technique. S2 


\subsection{Missing Early Mobilisation}

Several surgeons commented that early mobilisation was missing from the Guideline, despite its perceived ability to effectively prevent VTE.

There is no mention of early mobilisation as an effective means of thromboprophylaxis. S104

No mention of early mobilisation - probably most important. $S 216$

Early mobilisation \& short operative time [are] probably most effective to protect against DVT. S208

I use early mobilisation as best prophylaxis for VTE...S225

Early mobilisation makes chemoprophylaxis unnecessary. S30

Compliance with injections [is] difficult. Early mobilisation more important. S146

\subsection{Variety of Options and Risk Stratification}

There are many thromboprophylaxis options available to orthopaedic surgeons in the new ACCP Guideline; and there were mixed reactions to the large variety. Those that welcomed the range generally did so whilst simultaneously commenting positively on the Guideline only making recommendations and not dictating practice to surgeons.

Appropriate: Consideration of choice of wide array of $R x . S 98$

Best aspect is leaving choice to the doctor. S175

Appropriate: Leaves choice of chemoprophylaxis (including aspirin) to surgeon. $S 184$

Appropriate: RECOMMENDATION of several different options (including aspirin) for the treating doctor to choose from, based on the individual patients needs and relative risk of PE. S79

Concept of stratification of risk of DVT or PE is crucial. To subject ALL patients to risky prophylaxis makes no sense. $S 30$

Those surgeons who did not welcome the broad range of options described them as being 'too broad', 'a bit confusing', self-contradictory, and 'not answer[ing] the problem'.

Extremely frustrated [that] no consensus [was] achieved - especially as have had 2 PEs with calf compression / clexane / early mobilisation. S107

The wide range of options suggest[s] none is particularly effective. S35

'adding IPCD' contradicts 'or IPCD'. S63

I also feel [the guidelines are] too aggressive if recommended for standard, but major orthopaedic operations. Basically nothing helps other than early mobilisation! The rest is defensive, more than anything scientific! S165

None are inappropriate, but they are vague... S158

\subsection{Authorship}

Seven surgeons questioned the appropriateness of cardiothoracic physicians making recommendations for orthopaedic patients.

Inappropriate: The fact that PHYSICANS are trying to understand + Rx surgical patients. S165 
They are a group of arrogant dumb physicians who caused all the problems in the beginning by telling the American orthopods [orthopaedic surgeons] what they 'should' do. The Mayo tried their foolish protocol which caused [a] large number of bleeding issues. The 9th edition, are better, but why would[n't] I follow AAOS instead? S47

One participant commented that 'bitter experiences' had taught them 'not to trust physicians', while others generally commented that physicians are ill-positioned to appreciate postoperative complications in orthopaedic patients.

They don't see the wound complications, stiffness, thickening of soft tissues and scarring. S220 Physicians don't realise the morbidity of p[ost]o[perative] bleed / bruise -> stiffness of joint, painful scars, increased sepsis. S222

Many of us have had colleagues sued by so called experts who have never had to care for joint replacement patients and don't understand the complications of chemoprophylaxis and the subsequent effect on patient outcomes. S224

DVT prophylaxis for surgery is NOT understood by physicians and we have started using our own guidelines and tell the physicians to... S46

Evidence of outcomes of complications tends to be overlooked if assumption by CHEST etc. is only thrombotic complications are life threatening. 885

In addition to the negative comments made about the pharmaceutical industry biasing study outcomes, similar assertions were made about authors of the ACCP Guideline being 'sponsored' and having 'gross conflict[s] of interest'.

Do they have alternative motives to make this suggestion? Who is funding them? S213

The consultants who make the recommendations are recipients of research funding and I don't believe their opinions are without bias. S220

I think these people are compromised. S3

A lingering suspicion that physicians making these recommendations are not completely unbiased in their recommendations. 552

Concern over funding links of pharmaceutical industry to almost all senior medical advisers in this area. $S 214$

Similar allegations were also made about the ANZWP Guideline, and by inference, to the NHMRC Guideline; both participants below reported being very familiar with both of these guidelines.

ANZWP nominated themselves and are heavily sponsored by Sanofi - something they did not declare until edition 4. [The] only source document for [the] NHMRC [guideline] is the ANZ working Party. $S 8$

ANZWP - Funded by Clexane [enoxaparin] manufacturer! (Sanofi Aventis). S131 


\subsubsection{General Comments on Postoperative Complications}

\subsection{Venous Thromboembolism}

One participant commented that 'most orthopaedic surgeons don't care about DVT and/or nonfatal PE'. Potentially this may be because 'orthopaedic surgeons in general underestimate the risk as they do not necessarily hear about patients with PE or death', or maybe because they feel that 'surgical VTE is VERY different to non-surgical' VTE, and 'argue that fatal PE is not affected [by] leg prophylaxis'. Alternatively, some may find that the risk / benefit ratio for pharmacological agents is unacceptable.

My biggest concerns is that while chemoprophylaxis may/ may not reduce the risk of fatal PE, [and] fatality from other causes while using chemoprophylaxis is actually more likely i.e. fatal GIT [gastrointestinal] bleed from Warfarin etc. S174

Prophylaxis extended to 35 days increases the risk of complications proportional to time, but does not decrease the risk of significant PE correspondingly. S176

Fatal PE was different altogether; experiencing a patient die of PE made surgeons 'want to pull out all [the] stops'. Subsequent adverse experiences also impacted on how effectively they believed pharmacological agents prevent VTE, but did not dissuade them from prescribing it.

Before thromboprophylaxis I saw several patients die from PE. NIL since. S75

I have had 2 PEs with calf compression / clexane [enoxaparin] / early mobilisation. S107

I've anticoagulated all joint replacement patients. Generally if they are fit for operation, there is no contraindication to thromboprophylaxis. Even with this protocol, small number of patients $\rightarrow$ a VT[E]. Not had a fatal PE. But have had PE. S86

\subsection{Bleeding}

While bleeding is a natural complication of any surgery, it was acknowledged that the risk will logically be increased by the use of pharmacological agents.

\section{All chemoprophylaxis have potential for significant bleeding. $S 130$}

Concern was expressed that the true incidence of thromboprophylaxis associated postoperative bleeding is much higher than reported in the literature, with some participants commenting that it is 'never reported' and is 'underestimated'; conversely others commented that 'bleeding risk is overstated'. Nevertheless, surgeons commented that blood loss in itself was not their primary concern with postoperative bleeding, but rather they were concerned about its perceived tendency to delay wound and joint healing.

...the morbidity of p[ost]o[perative] bleed / bruise -> stiffness of joint, painful scars, increased sepsis. S222

I have found the use of LMWH in all arthroplasty patients to be associated with a disturbingly high incidence of bruising, persistent wound ooze, greater post op[erative] Hb [haemoglobin] drop \& an increased incidence of stiffness \& infection. S176 
I've tried many other chemicals [other than warfarin] and noted higher incidence of haemorrhage, bruising etc. joint stiffness increased, sepsis increased. S151

Although there are bleeding risks associated with thromboprophylaxis use, several surgeons commented that the benefits of using it outweighed these risks.

While I do not believe there is conclusive evidence for the prevention of fatal PE, the balance is for routine prophylaxis. $S 196$

Chemical thromboprophylaxis WORSENS [the] outcome of total join arthroplasty, but reduces death - BALANCE. S202

Rarely does it [bleeding] lead to significant wound issues. PE can be fatal! S162

It is a balance between safety for patients: to prevent clots vs. danger of bleeding. S175

While several participants noted that every pharmacological agent's risk profile is different, one commented that 'despite all of the literature, studies, etc. we still do not have a safe thromboprophylaxis agent'.

\subsection{Survey Comparison}

As noted previously, guidelines have decreased the strength of their recommendations over the years. When comparing ASA members' responses between the two surveys conducted two years apart (2010 and 2012), members were slightly more polarised in their answers, with a trend towards members becoming more confident regarding the ability of thromboprophylaxis to effectively prevent or not prevent fatal PE (Table 36).

Table 36. ASA members' opinions on efficacy of pharmacological prophylaxis (\%) ( $n=25$ and 48 respectively).

\begin{tabular}{|l|l|l|l|}
\hline Pharmacological agents... & Chapter $\mathbf{6}$ & Chapter $\mathbf{9}$ & $\mathbf{p}$ \\
\hline Do not decrease fatal PE & 24.0 & 27.0 & \\
\hline Potentially decrease fatal PE & 64.0 & 56.3 & 0.8 \\
\hline Definitely decrease fatal PE & 12.0 & 16.7 & \\
\hline
\end{tabular}

The top three factors that surgeons reported would prompt them to prescribe thromboprophylaxis were identical between both studies (Table 37). Surgeons in both surveys almost universally agreed that a history of VTE would prompt them to prescribe thromboprophylaxis. There were, however, significant differences between the proportion of surgeons who would be prompted to prescribe thromboprophylaxis for patients who were obese, had a history of thrombophlebitis or vein surgery, or whose arthroplasty surgery took more than 2 hours. 
Table 37. Patient factors that prompt surgeons to prescribe pharmacological prophylaxis (\%).

\begin{tabular}{|l|l|l|l|}
\hline & $\begin{array}{l}\text { Chapter } \mathbf{6} \\
(\mathrm{n}=25)\end{array}$ & $\begin{array}{l}\text { Chapter 9 } \\
(\mathrm{n}=170)\end{array}$ & $\mathbf{p}$ \\
\hline Age $<70$ years & 16.0 & 32.9 & 0.09 \\
\hline Age $\geq 70$ years & 20.0 & 35.9 & 0.12 \\
\hline Current smoker & 28.0 & 53.5 & $<0.05$ \\
\hline General anaesthesia & 12.0 & 30.0 & 0.06 \\
\hline HRT & $52.0 *$ & $65.3 *$ & 0.20 \\
\hline Moderate obesity (BMI $>30)$ & 32.0 & 54.7 & $<0.05$ \\
\hline Morbid obesity (BMI $>40)$ & 36.0 & 75.3 & $<0.05$ \\
\hline Preoperative Infection & 12.0 & 17.6 & 0.48 \\
\hline Previous thrombophlebitis & 28.0 & 57.1 & $<0.05$ \\
\hline Previous vein surgery & 20.0 & 40.6 & 0.05 \\
\hline Previous VTE & $96.0 *$ & $96.5 *$ & 0.90 \\
\hline Prolonged preoperative immobility & $44.0 *$ & $78.2 *$ & $<0.05$ \\
\hline Surgery $>$ 2 hours & 24.0 & 50.6 & $<0.05$ \\
\hline
\end{tabular}

NB: * indicates one of the top three factors.

Two of the top three factors that surgeons indicated would prevent them from prescribing thromboprophylaxis for their patients were identical in the two studies, namely the perception that pharmacological prophylaxis is not superior to mechanical prophylaxis and bleeding concerns (Table 38).

Table 38. Factors that limit pharmacological prophylaxis use (\%).

\begin{tabular}{|l|l|l|l|}
\hline & $\begin{array}{l}\text { Chapter 6 } \\
(\mathrm{n}=25)\end{array}$ & $\begin{array}{l}\text { Chapter 9 } \\
(\mathrm{n}=221)\end{array}$ & $\mathbf{p}$ \\
\hline It increases the risk of infection at the operation site & 28.0 & $41.6^{*}$ & 0.18 \\
\hline It is not safe as it causes too much bleeding & $76.0^{*}$ & $44.3^{*}$ & $<0.05$ \\
\hline It is not superior to mechanical methods & $40 . *^{*}$ & $25.8^{*}$ & 0.13 \\
\hline It is too expensive & 12.0 & 4.1 & 0.08 \\
\hline It is too inconvenient & $48.0 *$ & 19.9 & $<0.05$ \\
\hline Patients will not be compliant & 12.0 & 17.6 & 0.47 \\
\hline Poor continuation of care from hospital to community & 16.0 & 10.4 & 0.40 \\
\hline Study evidence isn't applicable to real-world populations & 36.0 & 19.5 & $<0.05$ \\
\hline The risks of VTE are low & $40.0 *$ & 16.7 & $<0.05$ \\
\hline
\end{tabular}

NB: * indicates one of the top three factors.

Guideline familiarity between the two surveys was noticeably different. Of note, all surgeons in the first survey were familiar with the ASA Guideline. In contrast, only $62.3 \%$ were familiar with the ASA Guideline in the second survey $(\mathrm{p}<0.001)$. This is likely because all participants in the first survey were ASA members, whereas only 42 participants (18.6\%) in the second survey were ASA members. Of these respondents, $92.8 \%$ were familiar with the ASA Guideline - similar 
to the first survey. This is evident in Figure 29 below; however, it is important to note that although the ACCP is the only guideline to have been revised in the years between both surveys, surgeon familiarity with the contemporary guidelines was generally lower across the board.

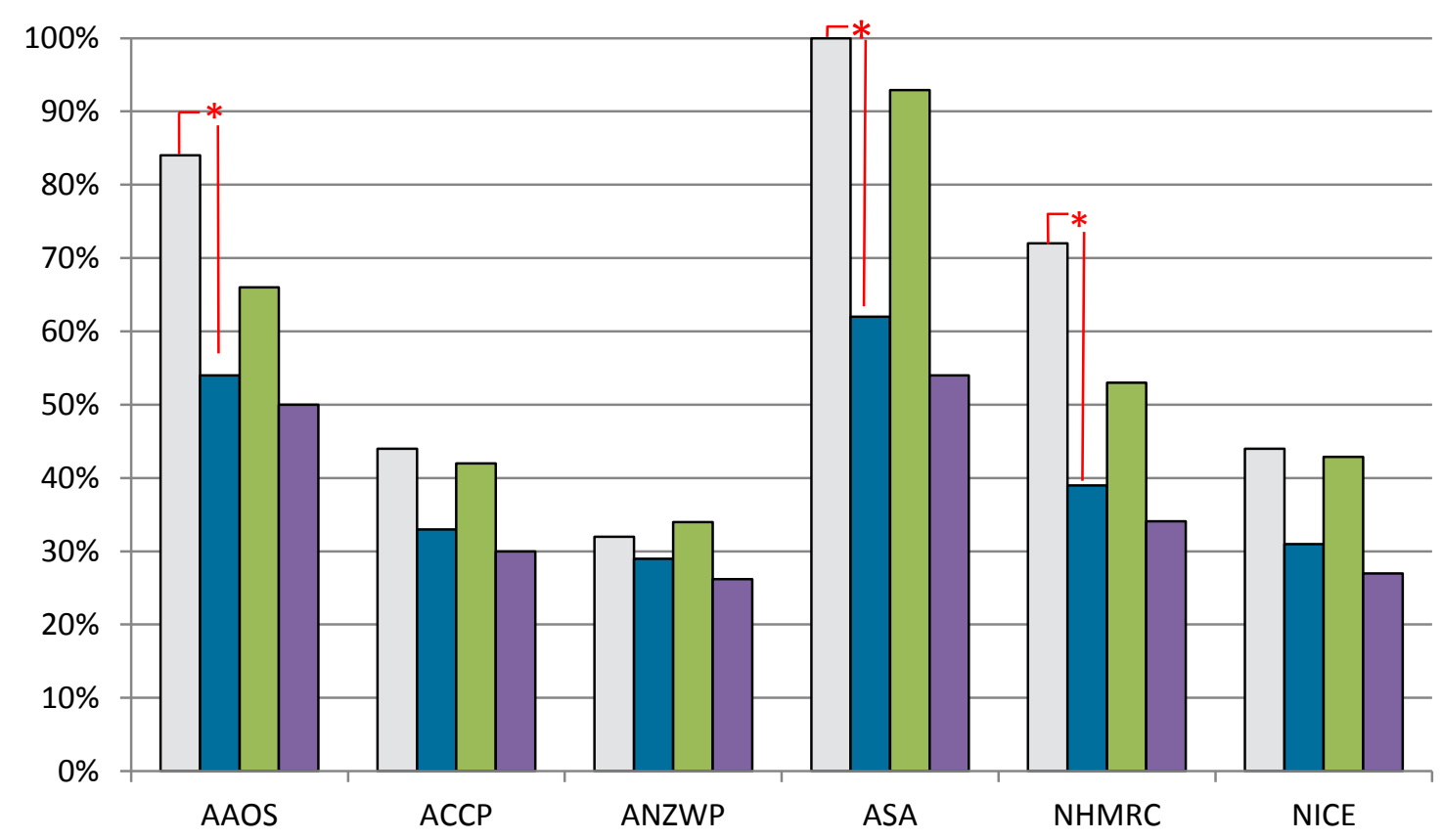

Figure 29. Proportion of surgeons very familiar with contemporary guidelines. $\square$ Chapter 6 data $\square$ Chapter 9 data, combined $\square$ Chapter 9 ASA members $\square$ Chapter 9 non-ASA members
*indicates statistically significant difference between $\square$ and $\square$ ( $p<0.05$ ).

\subsection{Discussion}

This survey demonstrated a lack of agreement amongst surgeons as to the appropriateness of the ACCP $9^{\text {th }}$ Ed. Guideline, and similar to the findings reported in Chapter 6 , to the general use of thromboprophylaxis following arthroplasty. ${ }^{206}$

As noted in 9.3 Methods the survey template used in this study was a shortened version of the survey used in the study outlined in Chapter 6. While some questions differed between the two surveys, a number were identical, allowing direct comparison between the findings of each survey. For example, in both surveys LMWH was the most popular agent of choice for surgeons, however the number of surgeons who preferred to use aspirin postoperatively in the current study was $75 \%$ lower than the previous survey ( $11.7 \%$ vs. $44.0 \%$ respectively). ${ }^{206}$ This may be because only ASA members (who had a tendency to prefer aspirin) were included in the previous survey and they only made up $23.0 \%$ of the response rate in this survey. 
Respondents described using a variety of thromboprophylaxis regimes in this survey, with many surgeons favouring a combination of different agents, instead of just one. Almost $15 \%$ of respondents said they preferred to use an anticoagulant during their patients' admission, and then switch to aspirin at discharge. Anderson et al reported this type of regime to be non-inferior to standard extended-therapy dalteparin use. ${ }^{209}$

Interestingly, surgeons who preferred to prescribe aspirin in this study tended to prescribe longer courses of prophylaxis than those who preferred to prescribe an anticoagulant \pm aspirin. These surgeons were also the ones most concerned about thromboprophylaxis associated bleeding; however it is likely one of the reasons they chose to prescribe aspirin over anticoagulants is the perceived lower bleeding risk.

Surgeons in both surveys generally preferred to prescribe thromboprophylaxis past the initial inpatient period and up to 2 to 6 weeks following surgery. There was a trend in both surveys to surgeons preferring to prescribe longer courses of thromboprophylaxis for hip arthroplasty patients compared to knee arthroplasty patients.

Despite their lack of original familiarity with them, surgeons in both surveys alleged that the evidence behind the guidelines (NHMRC Guideline in the previous study, the ACCP $9^{\text {th }}$ Ed. Guideline in this study) were founded on an inappropriate evidence base or were inappropriately broad. Significantly, of all the themes identified in the qualitative data of the present study, this was the only one which had a significant number of surgeons $(n=54,32.0 \%, N=169)$ in agreement with one another. This suggests the need for significant research in this field targeting the efficacy and dangers of thromboprophylaxis in the real world, taking into account patients' comorbidities, surgical differences and multi-modal strategies implemented to reduce VTE. Until this type of data is available (and it may never be), it is likely there will be little general consensus as to what constitutes appropriate thromboprophylaxis for arthroplasty patients.

Response rates to postal surveys are low and probably declining, almost certainly leading to unknown levels of bias. ${ }^{210}$ Health professionals, in particular doctors, are considered to be a problematic population from which to collect survey data, with average response rates from doctors reported to be around $60 \% .{ }^{210}$ It has been demonstrated that as response rates decrease, the risk for nonresponse bias increases, which is particularly relevant with a topic as sensitive as thromboprophylaxis following hip and knee arthroplasty. ${ }^{202}$ It is possible then, that non-responders would have been even less likely to agree with the ACCP Guideline than responders; however, only a follow up with non-responders would be able to confirm this. 


\section{Chapter Ten: Concluding Discussion}

Clinical practice guidelines are regularly disseminated by professional bodies, each designed to improve and optimise thromboprophylaxis use in hospitalised patients. Despite the ready availability of these guidelines, the National Institute of Clinical Studies has described 'appropriate prophylaxis' as 'often underused' in Australian patients, potentially leaving them exposed to a higher risk of VTE. ${ }^{10}$ The studies outlined in this thesis were conducted to describe and explore thromboprophylaxis prescribing practices and the factors that influence them. In total, six individual studies and one data reanalysis were conducted to achieve this aim.

The interviews identified that prescribers prefer to tailor therapy to individual patients - a practice known as personalised prescribing. The reviews identified that prophylaxis is routinely (and optimally) used in the immediate inpatient period, and anticoagulants (particularly LMWH) are prescribed preferentially over antiplatelets. On average, arthroplasty patients stay in hospital for five to six days postoperatively, and typically receive as many days of prophylaxis. Whilst prescribing is routine in hospital, the reviews identified that the provision of thromboprophylaxis at discharge is not.

There are many factors that influence whether a surgeon prescribes thromboprophylaxis for their patient at discharge or not. The surveys identified that surgeons were more likely to prescribe thromboprophylaxis for patients with a previous history of VTE; for smokers; for obese patients (BMI>30); for patients who were immobile for a prolonged period of time preoperatively; and for patients who had an operation time over two hours. Surgeons were less likely to prescribe thromboprophylaxis at discharge if they perceived it to be inconvenient for patients; perceived VTE risk as too low to warrant it; or perceived thromboprophylaxis to be unsafe due to bleeding risk. The interviews confirmed that instead of basing their opinion on study data and guidelines, surgeons' prescribing was greatly shaped by their training and collective experience of postoperative complications. Notably, some surgeons described being 'relatively ignorant of what's actually happening' in practice; this could result in a falsely high or low perception of their respective complication rates, thereby significantly influencing their prescribing practices.

Although surgeons reported good familiarity with the relevant literature, there was a general sense of mistrust, and the perception amongst some surgeons that study data does not correlate well with real-world populations. In particular, the use of asymptomatic clots as a surrogate measure for symptomatic VTE, and industry funding of studies and/or study authors negatively influenced surgeons' opinion of study data. Furthermore, some surgeons commented that there had been 
significant changes in the pre-, peri- and postoperative care of arthroplasty patients over the years, thereby making old studies out-dated and their findings seemingly obsolete.

In 2012 Molnar et al reported that orthopaedic surgeons' thromboprophylaxis prescribing was influenced by medico-legal concerns; that is, that surgeons were prescribing defensively. ${ }^{121}$ Surgeons in the interviews and surveys included in this thesis commented that guidelines can create a medico-legal dichotomy: on the one hand they can assist in guarding against medico-legal concerns by outlining what is 'middle of the road standard care'; on the other, they hold the potential to impose a medico-legal risk to surgeons who disagree (and practise in discordance) with their recommendations.

That guidelines have changed considerably over the years in their recommendations is the result of both methodology changes and an increase in the amount of study data available. Such changes in established evidence based recommendations are common, and occur across all classes of medical practice; the term coined for these changes is 'medical reversal'. ${ }^{211}$ One of the most commonly cited examples of medical reversal can be seen in the recommended care of cardiac patients. Percutaneous coronoary interventions $(\mathrm{PCI})$ are procedures conducted to revascularise areas of the heart that have been starved of blood flow due to coronary artery occlusions. The use of PCI on stable occlusions (i.e. not in the emergency setting) has been an established practice internationally, as it was perceived to improve patient survival and quality of life. However, recent studies have determined that reperfusion with PCI does not reduce the occurrence of death or disease progression in these patients, and may actually increase the risk of adverse events. ${ }^{211-215}$

The most common reason for medical reversal is that practice recommendations are often based on premature, inadequate, biased or conflicted evidence. ${ }^{216}$ "Another challenge is that conflicts of interest are common in medical research, leading many to question the validity and integrity of results from industry-sponsored trials' ${ }^{217}$ Larger, more robust studies that are conducted independent of industry funding and sponsorship are required to overcome this barrier. Notably, one reason why surgeons said they were hesitant to start prescribing novel oral agents was the concern that there is insufficient independent post-marketing surveillance data available to confirm their efficacy and safety in the real-world setting.

That guidelines have decreased in the strength of their recommendations suggests inconsistencies in study data, resulting in professional bodies becoming 'unclear about which prophylactic strategy (or strategies) is/are optimal or suboptimal'. ${ }^{166}$ Disputed or unclear evidence have been identified as barriers to guideline adoption. ${ }^{175,177}$ Significantly, the uncertainty influences the definition of what is appropriate, and therefore the degree to which evidence-practice gaps are perceived to exist. This is evident in the reanalysis outlined in Chapter 8, wherein a simple change in yardstick from the ACCP $7^{\text {th }}$ Ed. Guideline to the $9^{\text {th }}$ Ed. Guideline resulted in a six-fold perceived 'improvement' in thromboprophylaxis use. 
In summary, there are many factors that influence thromboprophylaxis prescribing. Of all of these factors, knowledge and communication are the most significant. To date there is no clear consensus on what constitutes appropriate thromboprophylaxis, and surgeons are not fully informed of their postoperative complication rates. Furthermore, many health professionals are unfamiliar with guideline recommendations and GPs said they were often ignorant of hospital/surgeon preferences for the post-discharge care of arthroplasty patients, when thromboprophylaxis is least likely to be prescribed. The recent advent of electronic discharge summaries that are emailed to GPs within 24 hours of patient discharge will likely assist greatly in keeping Tasmanian-based GPs informed about post-discharge care plans; however, in order for thromboprophylaxis to be optimised, all knowledge and communication barriers must be addressed.

Based on my findings, I recommend the formation of a local (or preferably national) database to collect and report back postoperative complication rates for arthroplasty patients to surgeons and hospitals. The collection of patient factors, operation factors and thromboprophylaxis use in such a database would assist to clarify how thromboprophylaxis influences postoperative complications in different patient groups, in the real-world setting. This information would also elucidate what constitutes appropriate thromboprophylaxis in different patient groups, and would naturally inform forthcoming national guideline editions, making recommendations more acceptable for surgeons. Lastly, guideline dissemination strategies need to be developed (or enhanced where they exist) to ensure all health professionals have ready knowledge and access to national guideline recommendations as they are developed. Simple strategies could include the dissemination of one to two page summaries from large guidelines such as the NHMRC Guideline. Such summaries would ideally include a decision aid tool, and relevant introduction or discussion to address surgeons' concerns, as suggested in Appendix E. Together these strategies will improve guideline recommendations, ensure optimal uptake of these recommendations and, most importantly, secure the best outcomes for arthroplasty patients. 
APPENDICES 


\section{APPENDix A}

\section{GUIDELINE RECOMMENDATION GRADINGS}

This appendix contains grading recommendations for the following guidelines:

- the AAOS Guideline (2007),

- $\quad$ the ACCP $8^{\text {th }}$ Guideline (2008),

- the ACCP $9^{\text {th }}$ Ed. Guideline (2012), and

- the NHMRC Guideline (2009). 


\section{AAOS Grading Recommendations (2007)}

"Each guideline recommendation was graded using the following system:

A: Good evidence (Level I Studies with consistent finding) for or against recommending intervention.

B: Fair evidence (Level II or III Studies with consistent findings) for or against recommending intervention.

C: Poor quality evidence (Level IV or V) for or against recommending intervention.

Level I evidence is from high quality randomized clinical trials (e.g. a randomized trial comparing revision rates in patients treated with cemented and uncemented total hip arthroplasty).

Level II evidence is from cohort studies (e.g. revision rates in patients treated with uncemented total hip arthroplasty compared with a control group of patients treated with cemented total hip arthroplasty at the same time and institution).

Level III evidence is from case-control studies (e.g. the rates of cemented and uncemented total hip arthroplasty in patients with a particular outcome called "cases"; i.e. revised total hip arthroplasty, are compared to those who did not have outcome, called "controls"; i.e. non-revised total hip arthroplasty).

Level IV evidence is from an uncontrolled case series (e.g. a case series of patients treated with uncemented total hip arthroplasty).

Level V evidence is from expert opinion. The table is relatively simple with four types of studies and five levels. The actual criteria for assignment is a little more complicated, and for interested readers, are contained in the cells of the table."156 


\section{ACCP $8^{\text {th }}$ Ed. Recommendations (2008)}

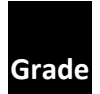

Grade of Recommendation ${ }^{*}$

1A Strong recommendation; high-quality evidence.

1B Strong recommendation moderate-quality evidence.

1C Strong recommendation; low or very lowquality evidence.

2A Weak recommendation; high-quality evidence.

2B Weak recommendation moderate-quality evidence.

\section{C Weak} recommendation; low or very lowquality evidence.

\section{Benefit vs Risk} and Burdens

Desirable effects clearly outweigh undesirable effects, or vice versa

Desirable effects clearly outweigh undesirable effects, or vice versa

\section{Desirable effects} clearly outweigh undesirable effects, or vice versa

Desirable effects closely balanced with undesirable effects

Desirable effects closely balanced with undesirable effects

\section{Desirable effects closely balanced with undesirable effects}

Methodologic Quality of

Supporting Evidence

Consistent evidence from RCTs without important limitations or exceptionally strong evidence from observational studies

\section{Evidence from RCTs with} important limitations (inconsistent results, methodological flaws, indirect or imprecise), or very strong evidence from observational studies

\section{Evidence for at least one}

critical outcome from observational studies, case series, or from RCTs with serious flaws or indirect evidence

Consistent evidence from RCTs without important limitations or exceptionally strong evidence from observational studies

Evidence from RCTs with important limitations (inconsistent results, methodological flaws, indirect or imprecise), or very strong evidence from observational studies

Evidence for at least one critical outcome from observational studies, case series, or from RCTs with serious flaws or indirect evidence
Implications

Recommendation can apply to most patients in most circumstances; further research is very unlikely to change our confidence in the estimate of effect

Recommendation can apply to most patients in most circumstances; higher quality research may well have an important impact on our confidence in the estimate of effect and may change the estimate

Recommendation can apply to most patients in many circumstances; higher-quality research is likely to have an important impact on our confidence in the estimate of effect and may well change the estimate

The best action may differ depending on circumstances or patient or society values; further research is very unlikely to change our confidence in the estimate of effect

Best action may differ depending on circumstances or patient or society values; higher-quality research may well have an important impact on our confidence in the estimate of effect and may change the estimate

Other alternatives may be equally reasonable; higher-quality research is likely to have an important impact on our confidence in the estimate of effect and may well change the estimate

* "We use the wording we recommend for strong (Grade 1) recommendations and we suggest for weak (Grade 2) recommendations." 218 


\section{ACCP $9^{\text {th }}$ Ed. Recommendations (2012)}

Grade of

Recommendation

$1 \mathrm{~A}$

\section{Strong}

recommendation;

high-quality

evidence.

$1 \mathrm{~B}$

\section{Strong}

recommendation

moderate-quality

evidence.

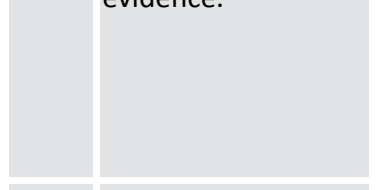

1C Strong

recommendation

low-or very-low-

quality evidence.

$2 \mathrm{~A}$

Weak

recommendation;

high-quality

evidence.

\section{Weak}

recommendation;

moderate-quality

evidence.
Benefit vs Risk

and Burdens

Benefits clearly outweigh risk and burdens or vice versa.

\section{Benefits clearly}

outweigh risk

and burdens or

vice versa.

\section{Benefits clearly}

outweigh risk

and burdens or

vice versa.

\section{Benefits closely}

balanced with

risks and burden.

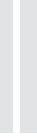

Benefits closely balanced with risks and burden.
Methodologic Strength of

Supporting Evidence

Consistent evidence from randomized controlled trials without important limitations or exceptionally strong evidence from observational studies.

\section{Evidence from randomized} controlled trials with important limitations (inconsistent results, methodologic flaws, indirect or imprecise) or very strong evidence from observational studies.

Evidence for at least one critical outcome from observational studies, case series, or randomized controlled trials, with serious flaws or indirect evidence.

Consistent evidence from randomized controlled trials without important limitations or exceptionally strong evidence from observational studies.

Evidence from randomized controlled trials with important limitations (inconsistent results, methodologic flaws, indirect or imprecise) or very strong evidence from observational studies.

\section{Implications}

Recommendation can apply to most patients in most circumstances. Further research is very unlikely to change our confidence in the estimate of effect.

Recommendation can apply to most patients in most circumstances. Higherquality research may well have an important impact on our confidence in the estimate of effect and may change the estimate.

Recommendation can apply to most patients in many circumstances. Higher-quality research is likely to have an important impact on our confidence in the estimate of effect and may well change the estimate.

The best action may differ depending on circumstances or patient or societal values. Further research is very unlikely to change our confidence in the estimate of effect.

Best action may differ depending on circumstances or patient or societal values. Higher-quality research may well have an important impact on our confidence in the estimate of effect and may change the estimate. 


\section{NHMRC Recommendations (2009)}

"Each recommendation is assigned a grade from ' $A$ ' to ' $D$ '.

- ' $\mathrm{A}$ ' refers to a recommendation based on a body of evidence that can be trusted to guide practice.

- ' $\mathrm{B}$ ' refers to a recommendation based on a body of evidence that can be trusted to guide practice in most situations.

- Grade ' $\mathrm{C}$ ' means that the body of evidence provides some support for the recommendation, but care should be taken in its application.

- Grade 'D' means that the body of evidence is weak and the recommendation should be applied only if considered appropriate after consideration of the clinical context.

Where no good-quality evidence was available but there was consensus among Committee members, consensus-based recommendations are given. Such recommendations are called Good Practice Points (GPPs)."17 


\section{APPENDIX B}

\section{Australian Hospital Protocols:}

This appendix includes the invitation letter sent to CNMs, as well as the survey itself. 
$16^{\text {th }}$ January 2012

To the Orthopaedic Clinical Nurse Manager,

I am writing to seek your assistance in completing a component of my $\mathrm{PhD}$ exploring factors influencing thromboprophylaxis prescribing following hip and knee arthroplasty. In particular, I would like to explore thromboprophylaxis protocols and their use in hospitals.

To assist me in this regard, I would like to invite you to complete a short online survey in conjunction with your Head of Orthopaedic Surgery. The survey should take less than 5 minutes to complete and all participants will be given the opportunity to participate in a draw for a \$250 gift voucher for the business/shop of their choice.

You can access the survey at: http://utas.edu.au/surveywebsite. If you have any questions you would like to ask before commencing this survey, please contact me via phone $(03)$ 62261083 or email ctaeed@utas.edu.au. All of the information that you provide will be treated confidentially.

In any reports or publications arising from this study, only the combined results will be published. If you have any concerns of an ethical nature, or complaints about the manner in which the project is conducted, please contact the Executive Officer of the Human Research Ethics Committee (Tasmania) Network (ph: 6226 7479, email: human.ethics@utas.edu.au; reference number $\mathrm{H} 0012250)$.

\section{Thank you for your assistance.}

Yours sincerely,

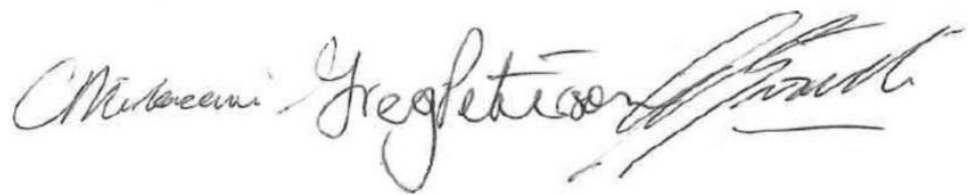

Corinne Mirkazemi $\mathrm{PhD}$ Candidate

\section{Greg Peterson} Professor of Pharmacy
Luke Bereznicki Senior Lecturer 
Survey Reminder:

Arthroplasty Thromboprophylaxis Protocols in Australian Hospitals

To the Orthopaedic Clinical Nurse Manager,

I am writing to seek your assistance in completing a component of my $\mathrm{PhD}$ exploring factors influencing thromboprophylaxis prescribing following hip and knee arthroplasty. In particular, I would like to explore the role of thromboprophylaxis protocols and their use in hospitals.

I would like to invite you to complete a short survey. The survey should take less than 5 minutes to complete and all participants will be given the opportunity to win a $\$ 250$ gift voucher for any nationally available business of their choice (e.g. Coles/Myer, Woolworths, Officeworks etc.). Participating hospitals can also opt in to receive a deidentified summary of the combined results of this study in order to compare against their own hospital.

All of the information that you provide will be treated confidentially. You can complete the survey by accessing it online at: http://tinyurl.com/6rmjfkn or by filling in the hard copy attached to this letter (and return it using the reply paid envelope). If you have any questions you would like to ask before commencing this survey, please contact me via phone (03) 62261083 or email ctaeed@utas.edu.au.

In any reports or publications arising from this study, only deidentified combined results will be published. If you have any concerns of an ethical nature, or complaints about the manner in which the project is conducted, please contact the Executive Officer of the Human Research Ethics Committee (Tasmania) Network (ph: 6226 7479, email: human.ethics@utas.edu.au; reference number H0012250).

\section{Thank you for your assistance.}

Yours sincerely,
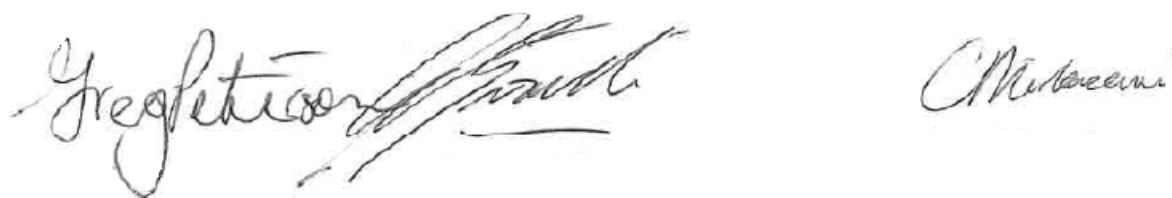

Greg Peterson

Luke Bereznicki

Emily Hansen

Professor of Pharmacy

Senior Lecturer

Research Fellow

Corinne Mirkazemi

PhD Candidate 


\section{ARTHROPLASTY THROMBOPROPHYLAXIS PROTOCOLS in AUSTRALIAN Hospitals}

\section{Hospital Demographics}

What state or territory is this hospital in?
- ACT
- NSW
- Queensland
- WA
- NT
South Australia
- Tasmania

Is this hospital a private or public hospital?

$$
\circ \text { Private hospital } \bigcirc \text { Public hospital }
$$

How many beds does this hospital accommodate? beds

Approximately how many surgeons conduct hip and knee arthroplasties at this hospital? Approximately how many hip arthroplasties are conducted each year? Approximately how many knee arthroplasties are conducted each year? per year surgeons per year

\section{Thromboprophylaxis Protocols}

Does this hospital have a formal thromboprophylaxis protocol for the orthopaedic unit? Yes / No (circle answer) If not, do individual surgeons have their own specific protocols?

If yes, is this protocol mandatory within the orthopaedic unit? (If possible, please include a copy of this protocol in your reply paid envelope.)

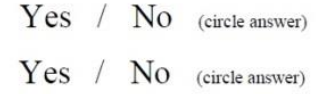

If yes, what is this protocol based on? (mark whichever answer(s) are applicable)

- NHMRC Thromboprophylaxis Guideline

- American Academy of Orthopaedic Surgeons (AAOS) Thromboprophylaxis Guideline

- Orthopaedic Unit or Hospital Consensus

Other (please specify)

- Arthroplasty Society of Australia Thromboprophylaxis Guideline

- Journal Publications

- Internal Hospital Data

- Unsure

If yes, are orthopaedic staff generally aware this protocol exists? Yes / No / Unsure (circle answer)

If yes, what do you estimate is the current adherence rate to this protocol for inpatient thromboprophylaxis? Hip arthroplasties: Knee arthroplasties: $\%$ receive thromboprophylaxis as per protocol $\%$ receive thromboprophylaxis as per protocol

If yes, what is the estimated current adherence rate to this protocol for thromboprophylaxis at discharge? Hip arthroplasties: $\%$ receive thromboprophylaxis as per protocol Knee arthroplasties: $\%$ receive thromboprophylaxis as per protocol

If yes, Does the hospital have policies in place to ensure adherence to the protocol? Yes / No (circle answer) If so, please describe how hospital ensures/encourages adherence to the protocol below.

Does the hospital have a post-discharge follow up for arthroplasty patients? Yes / No (circle answer) If yes, please describe what this post-discharge follow up entails below. 


\section{ARTHROPLASTY THROMBOPROPHYLAXIS PROTOCOLS in Australian Hospitals}

\section{Barriers to Protocol Uptake}

Do you believe any of the following perceptions are barriers to the uptake of a thromboprophylaxis protocol at this hospital?

\begin{tabular}{|l|c|c|c|c|}
\hline & Yes & No & Maybe & Not sure \\
\hline $\begin{array}{l}\text { There's no time to refer to the } \\
\text { protocol in practice }\end{array}$ & $\mathrm{O}$ & $\mathrm{O}$ & $\mathrm{O}$ & $\mathrm{O}$ \\
\hline $\begin{array}{l}\text { Protocols interfere with } \\
\text { professional autonomy }\end{array}$ & $\mathrm{O}$ & $\mathrm{O}$ & $\mathrm{O}$ & $\mathrm{O}$ \\
\hline $\begin{array}{l}\text { The protocol is not sufficiently } \\
\text { evidence based }\end{array}$ & $\mathrm{O}$ & $\mathrm{O}$ & $\mathrm{O}$ & $\mathrm{O}$ \\
\hline $\begin{array}{l}\text { VTE risk is too low to warrant } \\
\text { thromboprophylaxis }\end{array}$ & $\mathrm{O}$ & $\mathrm{O}$ & $\mathrm{O}$ & $\mathrm{O}$ \\
\hline $\begin{array}{l}\text { The hospital does not expect staff } \\
\text { to use the protocol }\end{array}$ & $\mathrm{O}$ & $\mathrm{O}$ & $\mathrm{O}$ & $\mathrm{O}$ \\
\hline $\begin{array}{l}\text { Patients are not compliant with } \\
\text { thromboprophylaxis }\end{array}$ & $\mathrm{O}$ & $\mathrm{O}$ & $\mathrm{O}$ & $\mathrm{O}$ \\
\hline $\begin{array}{l}\text { Thromboprophylaxis causes too } \\
\text { much bleeding }\end{array}$ & $\mathrm{O}$ & $\mathrm{O}$ & $\mathrm{O}$ & $\mathrm{O}$ \\
\hline
\end{tabular}

Thank you for completing this survey. Before you send it off to us, please tell us who had input into answering the questions of this survey:

- Orthopaedic Surgeon

- Head of Orthopaedic Unit

- Orthopaedic Clinical Nurse Manager

O Other (please specify)
- Orthopaedic Clinical Pharmacist

- Orthopaedic Registered Nurse

- Orthopaedic Registrar
Orthopaedic Resident

Orthopaedic Intern

Orthopaedic Physiotherapist

If you have any further comments on thromboprophylaxis prescribing following hip and knee arthroplasty, or on the survey in general please feel free to leave them below.

If you would be interested in the results of this study, please send your name and contact details to ctaeed@utas.edu.au or enter them in the text box below.

Name:

Email:

Similarly, if you would be interested in the possibility of discussing the topics covered in this survey further in the future (potentially via a short phone interview) please email ctaeed@utas.edu.au or enter your contact details below.

Name:

Email:

Lastly, if you would like to enter in the draw to win the new iPad or \$250 gift voucher please send your contact details to ctaeed@utas.edu.au or enter them below.

Name:

Email: 


\section{APPENDIX C \\ SURVEy OF AUSTRalian ORTHOPAEDIC SURGEONS}

This appendix includes the invitation letter sent to orthopaedic surgeons through the Arthroplasty Society of Australia, as well as the survey itself. 


\section{THROMBOPROPHYLAXIS FOLLOWING HIP AND KNEE ARTHROPLASTY}

Dear Surgeon,

The Unit for Medication Outcomes Research and Education (UMORE) at the School of Pharmacy, University of Tasmania would like to invite you to take part in a short online survey. This survey is a component of Corinne Mirkazemi's $\mathrm{PhD}$ exploring factors influencing thromboprophylaxis prescribing following hip and knee arthroplasty.

If you would like to take part in this study, simply access the questionnaire here. This survey should take less than 10 minutes to complete and all participants will be given the opportunity to participate in a draw for a brand new Apple iPad valued at \$629.

If you have any questions you would like to ask before commencing this survey, please contact Corinne via phone (03) 62261083 or email ctaeed@utas.edu.au. All of the information that you provide us with will be treated confidentially and all participants will remain anonymous. In any reports or publications arising from this study only the combined

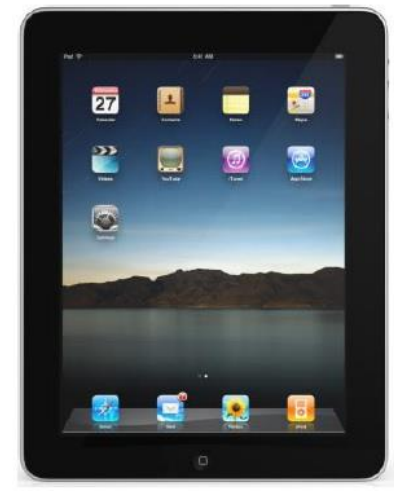
results will be published.

This study has been approved by the Tasmanian Health and Medical Human Research Ethics Committee. If you have concerns or complaints about the conduct of this study you should contact the Executive Officer of the HREC (Tasmania) Network on (03) 62267479 or email human.ethics@utas.edu.au. You will need to quote the reference number: H11265.

\section{Thank you for your assistance.}

Yours sincerely,

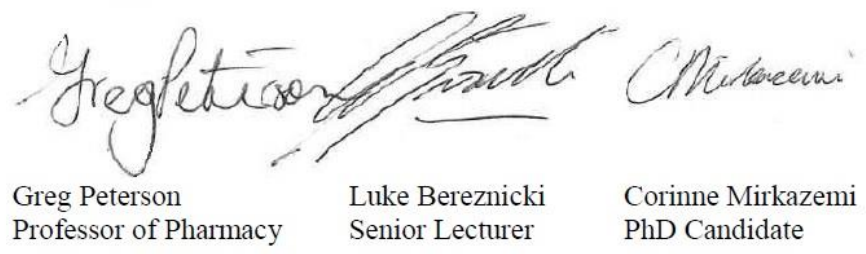




\section{THROMBOPROPHYLAXIS FOLLOWING HIP AND KNEE ARTHROPlasty - AN ONLINE SURVEY}

\section{Background}

1 What is your gender? $\square$ Male $\square$ Female

2 How many years have you been qualified as an orthopaedic surgeon? years

3 What state do you currently practise in?

4 Approximately how many patients do you perform the following surgeries on in an average month?

- Hip Arthroplasties per month

- Knee Arthroplasties per month

5 Do you conduct most of your arthroplasties in the private or public sector?

$\square$ Private sector predominantly $\quad \square$ Public sector predominantly $\square$ Both sectors equally

\section{Pharmacological Thromboprophylaxis}

6 Do you believe that thromboprophylaxis decreases the rate of fatal pulmonary embolism?
$\square$ Not at all
$\square$ Potentially decreases
Definitely decreases
Other (please specify)

7 Do you prescribe thromboprophylaxis for your patients?

$\square$ Yes

$\square$ No (go to question 12)

8 If yes, what agent do you prescribe most often? (Please choose only one of the following)
Aspirin
Dabigatran
Fondaparinux
Low Molecular Weight Heparin

$\square$ Rivaroxaban

$\square$ Standard Heparin

$\square$ Warfarin $\square$ Other (please specify)

9 Approximately how many of your arthroplasty patients receive this agent postoperatively? $\%$ of arthroplasty patients

10 What is your second preferred thromboprophylaxis agent? (Only if answer to \#9<100\%)
Aspirin
Dabigatran
Fondaparinux
Low Molecular Weight Heparin
$\square$ Rivaroxaban
$\square$ Standard Heparin $\square$ Warfarin
$\square$ None
$\square$ Other (please specify)

11 When do you prefer to stop pharmacological thromboprophylaxis following each procedure?

\begin{tabular}{|lcc|}
\hline & Knee Arthroplasty & Hip Arthroplasty \\
\hline When patient is mobile & $\square$ & $\square$ \\
\hline On discharge & $\square$ & $\square$ \\
\hline 14 days after surgery & $\square$ & $\square$ \\
\hline 28 to 35 days after surgery & $\square$ & $\square$ \\
\hline 3 months after surgery & $\square$ & $\square$ \\
\hline When GP decides & $\square$ & $\square$ \\
\hline Other & $\square$ & $\square$
\end{tabular}




\title{
THROMBOPROPHYLAXIS FOLLOWING HIP AND KNEE ARTHROPlasty - AN ONLINE SURVEY
}

\section{Inpatient Thromboprophylaxis}

12 Do you feel any of the following limit the prescribing of inpatient pharmacological thromboprophylaxis?
$\square$ It is not safe as it causes too much bleeding
$\square$ It is not superior to mechanical methods
$\square$ The risks of VTE are low
$\square$ Patients will not be compliant
$\square$ It is too expensive
Study evidence is not applicable to real-world populations
$\square$ There is poor continuation of care from hospital to community
$\square$ It increases the risk of infection at the operation site
$\square$ None of the above
$\square$ All of the above

13 Which factors would prompt you to provide pharmacological thromboprophylaxis during the inpatient period?
$\square$ Age $<70$ years
Age $\geq 70$ years
$\square$ Previous VTE
Current Smoker
$\square$ Previous thrombophlebitis
General anaesthesia
Hormone Replacement Therapy
Moderate Obesity (BMI > 30)
$\square$ Morbid Obesity (BMI > 40)
$\square$ Preoperative Infection
$\square$ Previous Vein Surgery
$\square$ Prolonged preoperative immobility
$\square$ Surgery $>2$ hrs duration
$\square$ None of the above
$\square$ All of the above
$\square$ Other (please specify)

\section{Post-discharge Thromboprophylaxis}

14 Do you feel any of the following limit the prescribing of pharmacological thromboprophylaxis after discharge?

$\square$ It is not safe as it causes too much bleeding

$\square$ It is not superior to mechanical methods

$\square$ Patients will not be compliant

$\square$ The risks of VTE are low

$\square$ Study evidence is not applicable to real-world populations
$\square$ There is poor continuation of care from hospital to community

$\square$ It increases the risk of infection at the operation site

$\square$ None of the above

All of the above

15 Which factors would prompt you to provide pharmacological thromboprophylaxis after discharge?
$\square$ Age $<70$ years
Age $\geq 70$ years
Current Smoker
$\square$ General anaesthesia
$\square$ Hormone Replacement Therapy
$\square$ Moderate Obesity (BMI > 30)
$\square$ Morbid Obesity (BMI > 40)
$\square$ Preoperative Infection

\author{
$\square$ Previous VTE \\ $\square$ Previous thrombophlebitis \\ $\square$ Previous Vein Surgery \\ $\square$ Prolonged preoperative immobility \\ $\square$ Surgery $>2$ hrs duration \\ $\square$ None of the above \\ $\square$ All of the above \\ $\square$ Other (please specify)
}




\section{THROMBOPROPHYLAXIS FOLLOWING HIP AND KNEE ARTHROPLASTY - AN ONLINE SURVEY}

\section{Case Studies}

The following pages include four case studies. Please answer the questions as closely as you can to your usual practice. If you feel you cannot do this with the options provided, please select the option 'other' to answer the first question in each case. Below is your first case study.

\section{Case Study 1}

A 90 yo female is admitted for left knee arthroplasty. She has a past medical history of irritable bowel syndrome and is hard of hearing. She denies any past history of VTE or bleeds. The patient is a lifetime non-smoker. She has a BMI of 19 and her creatinine clearance is $50 \mathrm{~mL} / \mathrm{min}$.

16 Assuming you would perform surgery on this patient, would you prescribe :

$\square$ Mechanical thromboprophylaxis only (Go to question 20)

$\square$ Pharmacological thromboprophylaxis only (Go to question 17)

$\square$ Both mechanical and pharmacological thromboprophylaxis (Go to question 17 \& 20)

$\square$ Neither

$\square$ Other (please specify)

17 Please indicate which drug you would prescribe

$\square$ Aspirin $\square$ Dabigatran $\square$ Fondaparinux $\square$ Low Molecular Weight Heparin

$\square$ Rivaroxaban $\square$ Standard Heparin $\square$ Warfarin $\square$ None $\square$ Other (please specify)

18 Please indicate what dose you would prescribe:

19 Please indicate the duration of therapy you would prescribe:
$\square 1$ to 7 days
$\square 22$ to 28 days
$\square 8$ to 14 days
$\square 29$ to 35 days
$\square 15$ to 21 days
$\square 35+$ days

20 Please indicate what form of mechanical thromboprophylaxis you would prescribe

$\square$ Intermittent pneumatic compression devices (IPC)

$\square$ Graduated compression stockings (GCS)

$\square$ Venous foot pump (VFP)

$\square$ Other (please specify) 


\section{THROMBOPROPHYLAXIS FOLLOWING HIP AND KNEE ARTHROPLASTY - AN ONLINE SURVEY}

\section{Case Study 2}

A 72 yo male is admitted for left hip arthroplasty. He has a past medical history of heartburn, gout, hypercholesterolaemia and diabetes (insulin-dependent). He denies any past history of VTE or bleeds. The patient is a lifetime non-smoker. He is overweight (BMI 31), and his creatinine clearance is $27 \mathrm{~mL} / \mathrm{min}$.

21 Assuming you would perform surgery on this patient, would you prescribe :
$\square$ Mechanical thromboprophylaxis only (Go to question 20)
$\square$ Pharmacological thromboprophylaxis only (Go to question 17)
$\square$ Both mechanical and pharmacological thromboprophylaxis (Go to question 17 \& 20)
$\square$ Neither
$\square$ Other (please specify)

22 Please indicate which drug you would prescribe
$\square$ Aspirin
$\square$ Dabigatran
Fondaparinux
Low Molecular Weight Heparin
$\square$ Rivaroxaban $\quad \square$ Standard Heparin
$\square$ Warfarin
None $\quad \square$ Other (please specify)

23 Please indicate what dose you would prescribe:

24 Please indicate the duration of therapy you would prescribe:
$\square 1$ to 7 days
$\square 22$ to 28 days
$\square 8$ to 14 days
$\square 29$ to 35 days
15 to 21 days
$\square 35+$ days

25 Please indicate what form of mechanical thromboprophylaxis you would prescribe

$\square$ Intermittent pneumatic compression devices (IPC) $\square$ Venous foot pump (VFP)

$\square$ Graduated compression stockings (GCS)

$\square$ Other (please specify)

\section{Case study 3}

A 56 yo female is admitted for right hip arthroplasty. She has a past medical history of pulmonary embolism, reflux and appendectomy. She is taking hormone replacement therapy (Premarin $625 \mathrm{mcg}$ daily) and denies any history of bleeds. The patient is a lifetime non-smoker. She is within a healthy weight range (BMI 21.5) and her creatinine clearance is $75 \mathrm{~mL} / \mathrm{min}$.

26 Assuming you would perform surgery on this patient, would you prescribe :

$\square$ Mechanical thromboprophylaxis only (Go to question 20)

$\square$ Pharmacological thromboprophylaxis only (Go to question 17)

$\square$ Both mechanical and pharmacological thromboprophylaxis (Go to question 17 \& 20)

$\square$ Neither

$\square$ Other (please specify)

27 Please indicate which drug you would prescribe
$\square$ Aspirin
$\square$ Fondaparinux
Low Molecular Weight Heparin
$\square$ Rivaroxaban $\quad \square$ Standard Heparin
$\square$ Warfarin
None
Other (please specify)

28 Please indicate what dose you would prescribe:

29 Please indicate the duration of therapy you would prescribe:
$\square 1$ to 7 days
$\square 22$ to 28 days
$\square 8$ to 14 days
$\square 29$ to 35 days
$\square 15$ to 21 days
$\square 35+$ days

30 Please indicate what form of mechanical thromboprophylaxis you would prescribe
$\square$ Intermittent pneumatic compression devices (IPC)
$\square$ Venous foot pump (VFP)
$\square$ Graduated compression stockings (GCS)
$\square$ Other (please specify) 


\section{THROMBOPROPHYLAXIS FOLLOWING HIP AND KNEE ARTHROPLASTY - AN ONLINE SURVEY}

\section{Case Study 4}

A 70 yo male is admitted for right knee arthroplasty. He has a past medical history of hypertension, asthma and haemorrhagic stroke. He denies any past history of VTE. The patient is a lifetime nonsmoker. He is within a healthy weight-range (BMI 23.1) and his creatinine clearance is $86 \mathrm{~mL} / \mathrm{min}$.

26 Assuming you would perform surgery on this patient, would you prescribe :

$\square$ Mechanical thromboprophylaxis only (Go to question 20)

$\square$ Pharmacological thromboprophylaxis only (Go to question 17)

$\square$ Both mechanical and pharmacological thromboprophylaxis (Go to question 17 \& 20)

$\square$ Neither

$\square$ Other (please specify)

27 Please indicate which drug you would prescribe

$\square$ Aspirin $\quad \square$ Dabigatran $\square$ Fondaparinux $\quad \square$ Low Molecular Weight Heparin

$\square$ Rivaroxaban $\quad \square$ Standard Heparin $\quad \square$ Warfarin $\quad \square$ None $\quad \square$ Other (please specify)

28 Please indicate what dose you would prescribe:

29 Please indicate the duration of therapy you would prescribe:
$\square 1$ to 7 days
$\square 8$ to 14 days
15 to 21 days
$\square 22$ to 28 days
$\square 29$ to 35 days
$\square 35+$ days

30 Please indicate what form of mechanical thromboprophylaxis you would prescribe

$\square$ Intermittent pneumatic compression devices (IPC) $\quad \square$ Venous foot pump (VFP)

$\square$ Graduated compression stockings (GCS)

$\square$ Other (please specify) 


\section{THROMBOPROPHYLAXIS FOLLOWING HIP AND KNEE ARTHROPLASTY - AN ONLINE SURVEY}

\section{Contemporary Guidelines}

31 Mark which statement best fits the level of familiarity you have with each contemporary thromboprophylaxis guideline.

\begin{tabular}{|c|c|c|c|}
\hline & I am very familiar with it & I have heard of it in passing & $\begin{array}{l}\text { I have not come across it } \\
\text { before }\end{array}$ \\
\hline $\begin{array}{l}\text { ANZ Working Party } \\
\text { Best Practice Guideline }\end{array}$ & $\square$ & $\square$ & $\square$ \\
\hline $\begin{array}{l}\text { NHMRC (NICS) Clinical } \\
\text { Practice Guideline }\end{array}$ & $\square$ & $\square$ & $\square$ \\
\hline $\begin{array}{l}\text { American Academy of } \\
\text { Orthopaedic Surgeons } \\
\text { Clinical Guideline }\end{array}$ & $\square$ & $\square$ & $\square$ \\
\hline NICE Guideline & $\square$ & $\square$ & $\square$ \\
\hline $\begin{array}{l}\text { Australian Arthroplasty } \\
\text { Society Guidelines }\end{array}$ & $\square$ & $\square$ & $\square$ \\
\hline
\end{tabular}

32 In late 2009 the National Health and Medical Research Council released their first Guideline for the prevention of VTE in patients admitted to Australian hospitals. This Guideline includes recommendations for the use of thromboprophylaxis after hip and knee arthroplasty (see summary $\underline{\text { here). }}$ (NB: hyperlink to a one page summary of recommendations - attached below)

Is there anything in these guidelines you feel is not appropriate?
$\square$ No
$\square$ Yes (Go to question 33)

33 Please outline below which aspects of the guideline you feel are not appropriate, and why.

34 Does your institution have a thromboprophylaxis protocol?

$\square$ No

Yes (Go to question 35)

35 Does your institution have processes to ensure compliance with the protocol?
$\square$ No
$\square$ Yes

Thank you for your time in completing this survey. Please feel free to enter any final thoughts below regarding thromboprophylaxis in general or this survey.

Would you be interested in the possibility of discussing the topics covered in this survey further in the future (potentially via a short phone interview)?

$\square$ Yes I would be interested.

No, thank you. 


\section{APPENDIX D}

\section{SEMI-STRUCTURED INTERVIEWS}

This appendix includes an example of the invitation letter and consent form sent to prospective participants, as well as an example of one of the early interview guides. NB: The interview guides were changed in an iterative approach after each interview. 
$9^{\text {th }}$ November 2010

\section{THROMBOPROPHYLAXIS FOLLOWING HIP AND KNEE ARTHROPLASTY}

Dear

We would like to invite you to take part in a study we are undertaking to explore the views of health professionals on the use of thromboprophylaxis following hip and knee arthroplasty. This project is a component of Corinne Mirkazemi's $\mathrm{PhD}$ examining the views of different health professionals on the use of thromboprophylaxis following hip and knee arthroplasty.

\section{What will it involve?}

Participating in this study will involve a 30 minute taped interview with Corinne, at a time and place that is convenient for you, either during normal business hours or after hours.

If you would like to take part, simply fill out the consent form attached and return it either using the enclosed reply-paid envelope, email (ctaeed@utas.edu.au) or fax (036226 7627). All of the information that you provide will be carefully managed to protect your confidentiality. The audio-recorded interview will be labelled using a code number and in any reports or publications arising from this study only the combined results will be published. Although some verbatim quotes might be reported to illustrate the findings, they will be labelled using a pseudonym and edited to remove identifying information.

If you have any questions about your participation in the study, please contact Corinne via phone (6226 1083) or email (ctaeed@utas.edu.aul). If you have any concerns of an ethical nature, or complaints about the manner in which the project is conducted, please contact the Executive Officer of the Human Research Ethics Committee (Tasmania) Network (Ph: 036226 7479, Email: human.ethics@utas.edu.au). You will need to quote the reference number H11165.

\section{Thank you for your assistance.}

Yours sincerely,
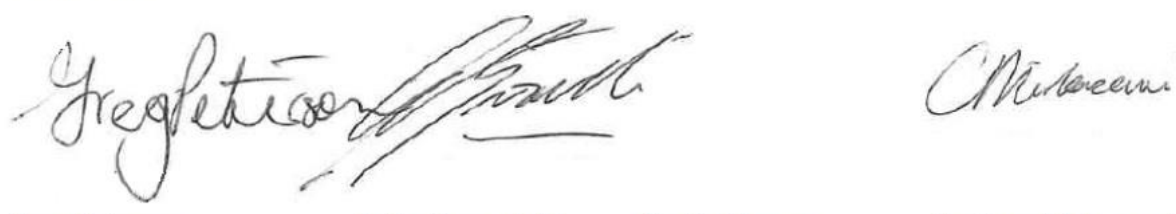

Greg Peterson Professor of Pharmacy
Luke Bereznicki Senior Lecturer
Emily Hansen Medical Researcher
Corinne Mirkazemi $\mathrm{PhD}$ Candidate 


\section{CONSENT FORM}

\section{THROMBOPROPHYLAXIS FOLLOWING HIP AND KNEE ARTHROPLASTY}

1 I have read and understood the introductory letter for this study.

2 The nature of the study has been explained to me, and any questions that I have asked have been answered to my satisfaction.

$4 \quad$ I understand that the study involves an audio-recorded interview with a researcher about my experiences and opinions prescribing thromboprophylaxis following hip and knee arthroplasty.

5 I understand any information provided in this study will be kept strictly confidential.

6 I understand all the data collected in this study will be stored in a locked cabinet or password protected computer in the School of Pharmacy and will be securely destroyed five years after publication of the data.

7 I have been informed that the results of the study may not be of any direct benefit to me.

8 I agree that research data gathered for the study may be published provided that pseudonyms will be used to ensure no individual data is identified.

9 I agree to participate in this study and understand that I am free to withdraw at any time without explanation or prejudice and to withdraw any unprocessed data previously supplied.

Name:

Date: $/ 2010$

Phone number: (to arrange a time and place for the interview)

Signature:

If you would like to be informed of the overall results of the study please mark this box and a copy of the results will be forwarded to your workplace.

\section{Statement by the researcher}

I have explained this study and the implications of participation in it to this volunteer and I believe that the consent is informed and that he/she understands the implications of participation.

Date: 


\title{
THROMBOPROPHYLAXIS FOLLOWING HIP AND KNEE REPLACEMENT SURGERY: The Surgeons Perspective
}

\author{
Surgeon Interview Outline
}

(N.B The schedule that follows is for a semi-structured interview, thus, the ordering of the questions will vary according to the way the interview evolves. Other questions not listed in this schedule may be asked to expand a topic if/as it emerges in the interview)

For this study, I'd like to examine some of the issues surrounding VTE prevention in patients who undergo total hip or knee replacement surgery. I am interviewing general practitioners and other health professionals in addition to other orthopaedic surgeons in order to get a broad picture of what current practice is and what factors influence it.

In these interviews with orthopaedic surgeons I am hoping to hear your opinions on what constitutes appropriate thromboprophylaxis following hip and knee replacement surgery, your opinion on the recent NHMRC guideline on VTE prevention and what factors influence your opinion and practise. All of the questions I ask will be pertaining to the hip and knee replacement patient group.

1. Roughly how many hip and knee replacement surgeries do you perform in a typical year?

2. What are your thoughts on VTE prophylaxis requirements after these surgeries?

3. What do you base your thoughts and practices on?

4. What are your thoughts on the current care flow from hospital to community for these surgical patients?

5. Who do you feel is primarily responsible for prescribing thromboprophylaxis for these patients after discharge from the hospital?

6. What suggestions do you have to improve the care of these patients, particularly relating to VTE prophylaxis?

Well those were all the questions I wanted to ask you. Is there anything you would like to add? Or anything you feel is important that we have left out?

Would you like to clarify any comments you have made? 


\section{APPENDIX E}

SUMMARY OF NHMRC THROMBOPROPHYLAXIS RECOMMENDATIONS 


\title{
NHMRC Thromboprophylaxis Recommendations for
}

\author{
Hip and Knee Replacement patients
}

Adequate hydration and early mobilisation are simple measures that should be applied as standard practice to prevent VTE. Other important options for VTE prophylaxis include pharmacological or mechanical methods.

The risk of VTE in patients must be balanced against the actual and perceived risks of pharmacological thromboprophylaxis and patients' tolerance of pharmacological (especially injectable) or mechanical prophylaxis. In particular, surgeons may be understandably reluctant to expose patients to the risk of excessive intra- or postoperative bleeding and the subsequent complications, especially in procedures such as joint replacement where bleeding can lead to severe infections and a need to explant prostheses.

By nature of its mechanism of action, pharmacological prophylaxis may increase the risk of surgical bleeding. As the evidence presented throughout this Guideline is mostly from randomised controlled trials, this may not be an accurate reflection of the incidence of bleeding outside the controlled trial context.

An assessment of bleeding risk is an essential step in deciding on appropriate thromboprophylaxis for individual patients. The final decision to provide thromboprophylaxis is a clinical decision based on the number and type of VTE risk factors balanced against risk of bleeding, and is made in consultation with the patient.

A VTE risk assessment should follow the following steps:

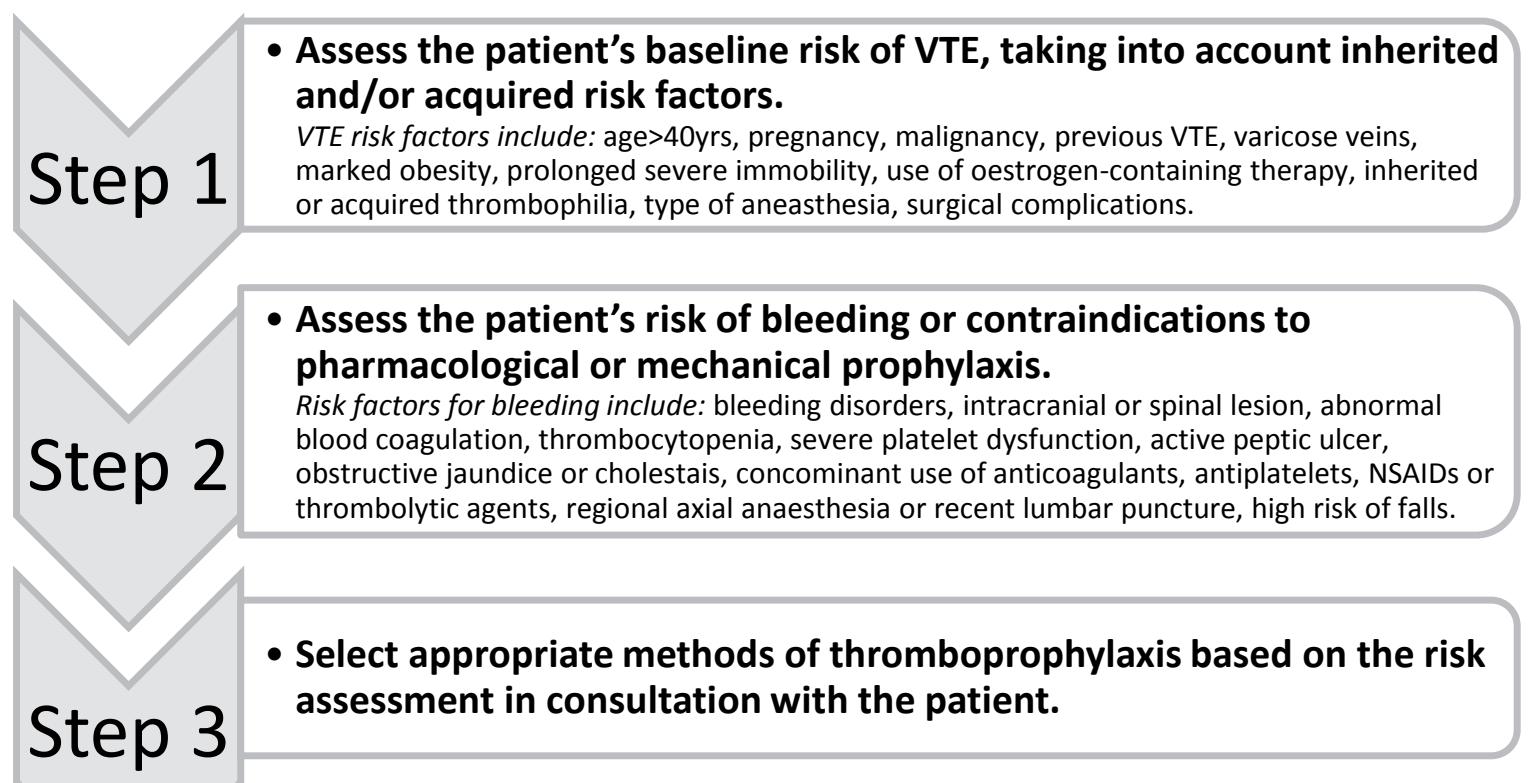


The recommendations below are intended to encapsulate the available evidence on the prevention of VTE. However, they should only be followed subject to the judgement of clinicians caring for individual patients and patients' own preferences.

\section{Total knee replacement}

Use thromboprophylaxis for all patients admitted to hospital for total knee replacement.

In the absence of contraindications, use one of the following agents for up to 14 days following surgery:

- low molecular weight heparin

- fondaparinux

- rivaroxaban

- dabigatran etexilate.

Use one of the following whether or not pharmacological thromboprophylaxis is used, until the patient is fully mobile:

- foot pump

- intermittent pneumatic compression.

Aspirin is not recommended as the sole pharmacological agent following total knee replacement.

Warfarin is not recommended for thromboprophylaxis following total knee replacement.

\section{Total hip replacement}

Use thromboprophylaxis for all patients admitted to hospital for total hip replacement.

In the absence of contraindications, use one of the following agents for up to 35 days following surgery:

- low molecular weight heparin

- fondaparinux

- rivaroxaban

- dabigatran etexilate.

Use graduated compression stockings, intermittent pneumatic compression or a foot pump until the patient is fully mobile, whether or not pharmacological thromboprophylaxis is used.

If possible, use graduated compression stockings with a foot pump where pharmacological thromboprophylaxis is not used.

Only use unfractionated heparin if recommended thromboprophylactic options are not available.

\section{\begin{tabular}{l|l} 
GRADE & DESCRIPTION
\end{tabular}}

A

B

Body of evidence can be trusted to guide practice

C

Body of evidence can be trusted to guide practice in most situations 


\section{APPENDIX F}

\section{SURGEONS AND THE ACCP 9TH ED. GUIDELINE}

This appendix includes the invitation letter sent to orthopaedic surgeons as well, as the survey itself. 


\section{SURVEY INVITE}

Dear Surgeon

I am writing to seek your assistance in completing the final component of my $\mathrm{PhD}$ exploring thromboprophylaxis prescribing following hip and knee arthroplasty. In particular I would like to explore surgeon perceptions of the recently released CHEST guidelines $9^{\text {th }} \mathrm{Ed}$. (2012).

This survey should take less than 10 minutes to complete and all participants will be given the opportunity to participate in a draw for a brand new Apple iPad valued at \$539. If you would like to take part in the survey, simply access it here: http://tinvurl.com/88vmwd4 alternatively fill in the hard copy attached to this letter and return it using the reply paid envelope.

If you have any questions you would like to ask before commencing this survey, please contact Corinne via phone (03) 62261083 or email

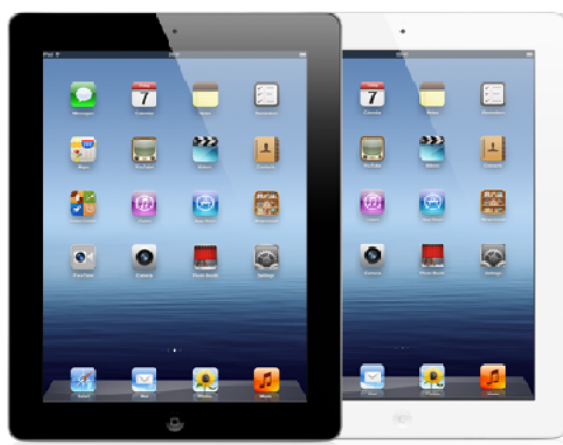
ctaeed@utas.edu.au. All of the information that you provide us with will be treated confidentially and all participants will remain anonymous. In any reports or publications arising from this study only the combined results will be published.

This study has been approved by the Tasmanian Health and Medical Human Research Ethics Committee. If you have concerns or complaints about the conduct of this study you should contact the Executive Officer of the HREC (Tasmania) Network on (03) 62267479 or email human.ethics@utas.edu.au. You will need to quote the reference number: H11265.

\section{Thank you for your assistance.}

Yours sincerely,

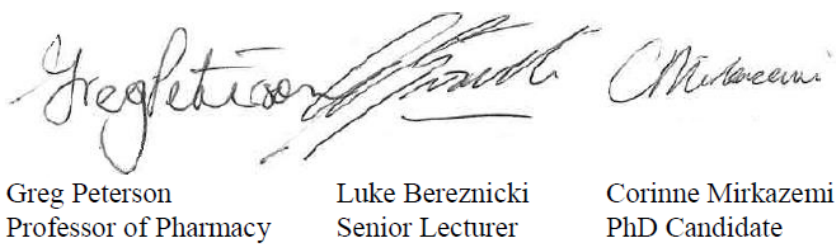




\section{THROMBOPROPHYLAXIS FOLLOWING HIP}

\section{Personal Demographics}

Are you a male or female?
- Male
- Female

How many years have you been qualified as an orthopaedic surgeon?

What state or territory do you practise in?
- ACT
- NSW
$\circ$ Queensland
- South Australia
$\circ$ WA
$\circ \quad$ NT

Do you conduct most of your arthroplasties in the private or public sector?
- Private sector predominantly
- Public sector predominantly
- Both sectors equally

\section{Pharmacological Thromboprophylaxis}

Do you believe that thromboprophylaxis decreases the rate of fatal PE?
- Not at all
- Potentially Decreases
- Definitely Decreases

Do you prescribe thromboprophylaxis for your patients?
$\circ$ Yes
$\circ$ No

If yes, when do you prefer to stop pharmacological prophylaxis?

Hip Arthroplasty:

Knee Arthroplasty:

\section{Contemporary Guidelines}

Mark which statement best fits the level of familiarity you have with each thromboprophylaxis guideline:

\begin{tabular}{|l|c|c|c|}
\hline & I am very familiar with it & I have heard of it in passing & I have not come across it \\
\hline ANZ Working Party & 0 & 0 & 0 \\
\hline NHRMC & 0 & 0 & 0 \\
\hline CHEST 12 th $^{\text {Ed }}$ & 0 & 0 & 0 \\
\hline $\begin{array}{l}\text { American Academy } \\
\text { of Orthopaedic } \\
\text { Surgeons }\end{array}$ & 0 & 0 & 0 \\
\hline $\begin{array}{l}\text { NICE Guideline } \\
\text { (UK) }\end{array}$ & 0 & 0 & 0 \\
\hline $\begin{array}{l}\text { Arthroplasty Society } \\
\text { Guidelines }\end{array}$ & 0 & 0 & 0 \\
\hline
\end{tabular}

In early 2012 the CHEST physicians released their $12^{\text {th }}$ Ed. Guideline for the prevention of VTE in patients admitted to hospitals. The guideline includes the following recommendations for hip and knee arthroplasty patients.

We recommend the use of one of the following rather than no antithrombotic prophylaxis: Low molecular weight heparin, fondaparinux, dabigatran, apixaban, rivaroxaban, low dose unfractionated heparin, adjusted dose vitamin $K$ antagonist, aspirin (Grade IB) or an inter-pneumatic compression device(IPCD) (Grade IC). We recommend the use of low molecular weight heparin in preference to the other agents we have recommended as alternatives (Grade $2 C / 2 B$ ), and in patients receiving pharmacological prophylaxis we suggest adding an IPCD during the hospital stay (Grade 2C). We suggest extending thromboprophylaxis for up to 35 days (Grade $2 B$ ). In patients at increased bleeding risk, we suggest an IPCD or no prophylaxis (Grade 2C). In patients who decline injections, we recommend using apixaban or dabigatran (Grade IB). 
THROMBOPROPHYLAXIS FOLLOWING HIP AND KNEE ARTHROPLASTY

Please outline any aspects of the CHEST $12^{\text {th }}$ Ed. recommendations that you feel are inappropriate

Please outline any aspects of the CHEST $12^{\text {th }}$ Ed. recommendations that you feel are appropriate

If you have any further comments on thromboprophylaxis prescribing following hip and knee arthroplasty, or on the survey in general please feel free to leave them below.

If you would be interested in the results of this study, please send your name and contact details to ctaeed@utas.edu.au or enter them below

Name: Email:

Similarly, if you would like to enter in the draw to win the new iPad or $\$ 250$ gift voucher please send your contact details to ctaeed@utas.edu.au or enter them below.

Name: Email: 


\section{REFERENCES}

1. Prevention of Venous Thromboembolism: Best Practice Guidelines for Australia \& New Zealand. 2007. http://www.racs.edu.au/media/19372/VTE_Guidelines.pdf . Accessed 10 April 2012.

2. Anderson Jr FA, Spencer FA. Risk factors for venous thromboembolism. Circulation. 2003;107(SUPPL. 23):I9-I16.

3. Maynard G, Stein J. Preventing Hospital-Acquired Venous Thromboembolism: A Guide for Effective Quality Improvement: Agency for Healthcare Research and Quality; 2008.

4. Cohen AT, Tapson VF, Bergmann JF, et al. Venous thromboembolism risk and prophylaxis in the acute hospital care setting (ENDORSE study): a multinational cross-sectional study. The Lancet. 2008;371(9610):387-394.

5. Hitos K, Fletcher JP. Venous thromboembolism and fractured neck of femur. Thrombosis and Haemostasis. 2005;94(5):991-996.

6. Hitos K, Fletcher JP. Venous thromboembolism following primary total knee arthroplasty. International Angiology. 2006;25(4):343-351.

7. Hitos K, Fletcher JP. Venous thromboembolism following primary total hip arthroplasty. International Angiology. 2009;28(3):215-221.

8. Eikelboom JW, Mazzarol A, Quinlan DJ, et al. Thromboprophylaxis practice patterns in two Western Australian teaching hospitals. Haematologica. 2004;89(5):586-593.

9. Wan S, Ting J, Olsen A, Croser J, Eikelboom JW. Thromboprophylaxis practice patterns in hip fracture surgery patients: Experience in Perth, Western Australia. ANZ Journal of Surgery. 2003;73(10):826-829.

10. NHMRC. Evidence-Practice Gaps Report: A review of developments 2004-2007. 2008;1:2427.

11. Kakkar AK, Cohen AT, Tapson VF, et al. Venous thromboembolism risk and prophylaxis in the acute care hospital setting (ENDORSE survey): Findings in surgical patients. Annals of surgery. 2010;251(2):330-338.

12. Burns R. Introduction to Research Methods. Melbourne: Longman Cheshire; 1994.

13. Cohen L, Manion L, Morrison KRB. Research Methods in Education. 5th ed. London: Psychology Press; 2000.

14. Risjord M, Moloney M, Dunbar S. Methodological triangulation in nursing research. Philosophy of the Social Sciences. 2001;31(1):40-59.

15. NHMRC. Incidence and Risk Factors for VTE in Western Australian Hospitals. 2005; http://www.nhmrc.gov.au/nics/nics-programs/vte-prevention-guideline/venousthromboembolism-prevention-program-\%E2\%80\%94-evidence-bas. Accessed 09/01/2013.

16. Lowe GDO. Common risk factors for both arterial and venous thrombosis. British Journal of Haematology. 2008;140(5):488-495.

17. NHMRC. Clinical Practice Guideline for the Prevention of Venous Thromboembolism in Patients Admitted to Australian Hospitals. Melbourne: National Health and Medical Research Council; 2009.

18. Kearon C. Natural history of venous thromboembolism. Circulation. 2003;107(SUPPL. 23):I22-I30.

19. White RH. The epidemiology of venous thromboembolism. Circulation. 2003;107(SUPPL. 23):I4-I8.

20. Grol R, Dalhuijsen J, Thomas S, In 'T Veld C, Rutten G, Mokkink H. Attributes of clinical guidelines that influence use of guidelines in general practice: Observational study. British Medical Journal. 1998;317(7162):858-861.

21. Wood KE. A History of Pulmonary Embolism and Deep Venous Thrombosis. Critical Care Clinics. 2009;25(1):115-131. 
22. Kroegel C, Reissig A. Principle mechanisms underlying venous thromboembolism: Epidemiology, risk factors, pathophysiology and pathogenesis. Respiration. 2003;70(1):7-30.

23. White RH. Identifying risk factors for venous thromboembolism. Circulation. 2012;125(17):2051-2053.

24. Husted H, Otte KS, Kristensen BB, Ørsnes T, Kehlet H. Readmissions after fast-track hip and knee arthroplasty. Archives of Orthopaedic and Trauma Surgery. 2010;130(9):1185-1191.

25. Keeney JA, Adelani MA, Nunley RM, Clohisy JC, Barrack RL. Assessing readmission databases: How reliable is the information? Journal of Arthroplasty. 2012;27(8 SUPPL.):7276.e72.

26. Pellegrini Jr VD, Donaldson CT, Farber DC, Lehman EB, Evarts CM. The Mark Coventry Award: Prevention of readmission for venous thromboembolism after total knee arthroplasty. Clinical Orthopaedics and Related Research. 2006(452):21-27.

27. Becker RC. The importance of VTE prevention after orthopaedic surgery. The Lancet. 2009;373(9676):1661-1662.

28. Access Economics. The burden of venous thromboembolism in Australia. Report for the Australia and New Zealand Working Party on the Management and Prevention of Venous Thromboembolism. 2008.

29. Wong P, Baglin T. Epidemiology, risk factors and sequelae of venous thromboembolism. Phlebology. 2012;27(SUPPL.2):2-11.

30. Ho WK, Hankey GJ, Eiketboom JW. The incidence of venous thromboembolism: A prospective, community-based study in Perth, Western Australia. Medical Journal of Australia. 2008;189(3):144-147.

31. Heit JA. Predicting the risk of venous thromboembolism recurrence. American Journal of Hematology. 2012;87(SUPPL. 1):S63-S67.

32. Hansson PO, Sörbo J, Eriksson H. Recurrent venous thromboembolism after deep vein thrombosis: Incidence and risk factors. Archives of Internal Medicine. 2000;160(6):769-774.

33. Baglin $\mathrm{T}$. What happens after venous thromboembolism? Journal of Thrombosis and Haemostasis. 2009;7(SUPPL. 1):287-290.

34. Zhu T, Martinez I, Emmerich J. Venous thromboembolism: Risk factors for recurrence. Arteriosclerosis, Thrombosis, and Vascular Biology. 2009;29(3):298-310.

35. White RH, Murin S, Wun T, Danielsen B. Recurrent venous thromboembolism after surgeryprovoked versus unprovoked thromboembolism. Journal of Thrombosis and Haemostasis. 2010;8(5):987-997.

36. Murin S, Romano PS, White RH. Comparison of outcomes after hospitalization for deep venous thrombosis or pulmonary embolism. Thrombosis and Haemostasis. 2002;88(3):407414.

37. Eichinger S, Weltermann A, Minar E, et al. Symptomatic Pulmonary Embolism and the Risk of Recurrent Venous Thromboembolism. Archives of Internal Medicine. 2004;164(1):92-96.

38. The EINSTEIN Investigators. Oral rivaroxaban for symptomatic venous thromboembolism. New England Journal of Medicine. 2010;363(26):2499-2510.

39. Kahn SR. The post-thrombotic syndrome: Progress and pitfalls. British Journal of Haematology. 2006;134(4):357-365.

40. Kahn SR, Hirsch A, Shrier I. Effect of postthrombotic syndrome on health-related quality of life after deep venous thrombosis. Archives of Internal Medicine. 2002;162(10):1144-1148.

41. Kahn SR, Ducruet T, Lamping DL, et al. Prospective evaluation of health-related quality of life in patients with deep venous thrombosis. Archives of Internal Medicine. 2005;165(10):1173-1178.

42. Lonner JH, Frank J, McGuire K, Lotke PA. Postthrombotic syndrome after asymptomatic deep vein thrombosis following total knee and hip arthroplasty. American journal of orthopedics (Belle Mead, N.J.). 2006;35(10):469-472.

43. Ashrani AA, Heit JA. Incidence and cost burden of post-thrombotic syndrome. Journal of Thrombosis and Thrombolysis. 2009;28(4):465-476.

44. Mant MJ, Eurich DT, Russell DB, Majumdar SR. Post-thrombotic syndrome after total hip arthroplasty is uncommon. Acta Orthopaedica. 2008;79(6):794-799. 
45. Ginsberg JS, Gent M, Turkstra F, et al. Postthrombotic syndrome after hip or knee arthroplasty: A cross- sectional study. Archives of Internal Medicine. 2000;160(5):669-672.

46. Cushman M, Tsai AW, White RH, et al. Deep vein thrombosis and pulmonary embolism in two cohorts: The longitudinal investigation of thromboembolism etiology. American Journal of Medicine. 2004;117(1):19-25.

47. Hooper GJ, Rothwell AG, Hooper NM, Frampton C. The relationship between the American Society of Anesthesiologists physical rating and outcome following total hip and knee arthroplasty: An analysis of the New Zealand Joint Registry. Journal of Bone and Joint Surgery - Series A. 2012;94(12):1065-1070.

48. Siddique RM, Siddique MI, Connors Jr AF, Rimm AA. Thirty-day case-fatality rates for pulmonary embolism in the elderly. Archives of Internal Medicine. 1996;156(20):2343-2347.

49. Comba F, Hidalgo IA, Buttaro M, Piccaluga F. Risk Factor Analysis for 30-Day Mortality After Primary THA in a Single Institution. HSS Journal. 2012;8(2):111-115.

50. Vulcano E, Gesell M, Esposito A, Ma Y, Memtsoudis SG, Gonzalez Della Valle A. Aspirin for elective hip and knee arthroplasty: a multimodal thromboprophylaxis protocol. International Orthopaedics. 2012:1-8.

51. Lapidus LJ, Ponzer S, Pettersson H, De Bri E. Symptomatic venous thromboembolism and mortality in orthopaedic surgery - An observational study of 45968 consecutive procedures. BMC Musculoskeletal Disorders. 2013;14.

52. Boeije H. A purposeful approach to the constant comparative method in the analysis of qualitative interviews. Quality and Quantity. 2002;36(4):391-409.

53. Australian Orthopaedic Association. National Joint Replacement Registry Annual Report. 2012;

https://aoanjrr.dmac.adelaide.edu.au/documents/10180/60142/Annual\%20Report\%202012. Accessed 08 January 2012.

54. Australian Orthopaedic Association. National Joint Replacement Registry Supplementary Report - Knee Demographics. 2012; https://aoanjrr.dmac.adelaide.edu.au/documents/10180/68968/KneeDemographicsReport_201 2.pdf. Accessed 08 January 2012.

55. Lieberman JR, Hsu WK. Prevention of venous thromboembolic disease after total hip and knee arthroplasty. Journal of Bone and Joint Surgery - Series A. 2005;87(9 I):2097-2112.

56. Borris LC. Barriers to the optimal use of anticoagulants after orthopaedic surgery. Archives of Orthopaedic and Trauma Surgery. 2008:1-5.

57. Kageyama K, Nakajima $\mathrm{Y}$, Shibasaki M, Hashimoto S, Mizobe T. Increased platelet, leukocyte, and endothelial cell activity are associated with increased coagulability in patients after total knee arthroplasty. Journal of Thrombosis and Haemostasis. 2007;5(4):738-745.

58. Lalmohamed A, Vestergaard P, Jansen PAF, et al. Prolonged outpatient vitamin K antagonist use and risk of venous thromboembolism in patients undergoing total hip or knee replacement. Journal of Thrombosis and Haemostasis. 2013;11(4):642-650.

59. Scalzitti DA. Evidence-based guidelines: Application to clinical practice. Physical Therapy. 2001;81(10):1622-1628.

60. White RH, Zhou H, Romano PS. Incidence of symptomatic venous thromboembolism after different elective or urgent surgical procedures. Thrombosis and Haemostasis. 2003;90(3):446-455.

61. Markovic-Denic L, Zivkovic K, Lesic A, Bumbasirevic V, Dubljanin-Raspopovic E, Bumbasirevic M. Risk factors and distribution of symptomatic venous thromboembolism in total hip and knee replacements: Prospective study. International Orthopaedics. 2012;36(6):1299-1305.

62. Bjørnarå BT, Gudmundsen TE, Dahl OE. Frequency and timing of clinical venous thromboembolism after major joint surgery. Journal of Bone and Joint Surgery - Series B. 2006;88(3):386-391.

63. O'Reilly RF, Burgess IA, Zicat B. The prevalence of venous thromboembolism after hip and knee replacement surgery. Medical Journal of Australia. 2005;182(4):154-159.

64. Quinlan DJ, Eikelboom JW, Dahl OE, Eriksson BI, Sidhu PS, Hirsh J. Association between asymptomatic deep vein thrombosis detected by venography and symptomatic venous 
thromboembolism in patients undergoing elective hip or knee surgery. Journal of Thrombosis and Haemostasis. 2007;5(7):1438-1443.

65. Joseph JE, Low J, Courtenay B, Neil MJ, McGrath M, Ma D. A single-centre prospective study of clinical and haemostatic risk factors for venous thromboembolism following lower limb arthroplasty. British Journal of Haematology. 2005;129(1):87-92.

66. Persson LM, Lapidus LJ, Lärfars G, Rosfors S. Asymptomatic Deep Venous Thrombosis is Associated with a Low Risk of Post-thrombotic Syndrome. European Journal of Vascular and Endovascular Surgery. 2009;38(2):229-233.

67. Wille-Jørgensen P, Jorgensen LN, Crawford M. Asymptomatic postoperative deep vein thrombosis and the development of postthrombotic syndrome. A systematic review and metaanalysis. Thrombosis and Haemostasis. 2005;93(2):236-241.

68. Falck-Ytter Y, Francis CW, Johanson NA, et al. Prevention of VTE in orthopedic surgery patients. Antithrombotic therapy and prevention of thrombosis, 9th ed: American College of Chest Physicians evidence-based clinical practice guidelines. Chest. 2012;141(2 SUPPL.):e278S-e325S.

69. Oldmeadow LB, McBurney H, Robertson VJ. Hospital stay and discharge outcomes after knee arthroplasty: Implications for physiotherapy practice. Australian Journal of Physiotherapy. 2002;48(2):117-121.

70. Husted H, Otte KS, Kristensen BB, Ørsnes T, Wong C, Kehlet H. Low risk of thromboembolic complications after fast-track hip and knee arthroplasty. Acta Orthopaedica. 2010;81(5):599-605.

71. Raphael M, Jaeger M, Van Vlymen J. Easily adoptable total joint arthroplasty program allows discharge home in two days. Canadian Journal of Anesthesia. 2011;58(10):902-910.

72. Cram P, Lu X, Kates SL, Singh JA, Li Y, Wolf BR. Total knee arthroplasty volume, utilization, and outcomes among medicare beneficiaries, 1991-2010. JAMA - Journal of the American Medical Association. 2012;308(12):1227-1236.

73. Fouilleron N, Wavreille G, Endjah N, Girard J. Running activity after hip resurfacing arthroplasty: A prospective study. American Journal of Sports Medicine. 2012;40(4):889-894.

74. Jacobs CA, Christensen CP, Berend ME. Sport activity after total hip arthroplasty: Changes in surgical technique, implant design, and rehabilitation. Journal of Sport Rehabilitation. 2009;18(1):47-59.

75. Eikelboom JW, Quinlan DJ, Douketis JD. Extended-duration prophylaxis against venous thromboembolism after total hip or knee replacement: A meta-analysis of the randomised trials. Lancet. 2001;358(9275):9-15.

76. Huo MH, Muntz J. Extended thromboprophylaxis with low-molecular-weight heparins after hospital discharge in high-risk surgical and medical patients: A review. Clinical Therapeutics. 2009;31(6):1129-1141.

77. Muntz J. Thromboprophylaxis in orthopedic surgery: how long is long enough? American journal of orthopedics (Belle Mead, N.J.). 2009;38(8):394-401.

78. Nair V, Kumar R, Singh B, Sharma A, Joshi G, Pathak K. Comparative study of extended versus short term thromboprophylaxis in patients undergoing elective total hip and knee arthroplasty in Indian population. Indian Journal of Orthopaedics. 2013;47(2):161-167.

79. Fisher WD, Gent M, Lassen MR, Kakkar AK, Eriksson B, Turpie AG. Timing of Symptomatic Venous Thromboembolism and Bleeding events After Total Hip Arthroplasty: A pooled Analysis of Rivaroxaban Studies. Journal of Bone and Joint Surgery - Series B. 2011;93(Supp II 90).

80. Buchan DS, Bajorek B. Incidence of venous thromboembolism and thromboprophylaxis after total hip or knee arthroplasty. Journal of Pharmacy Practice and Research. 2008;38(3):200204.

81. Cho KY, Kim KI, Khurana S, Bae DK, Jin W. Is routine chemoprophylaxis necessary for prevention of venous thromboembolism following knee arthroplasty in a low incidence population? Archives of Orthopaedic and Trauma Surgery. 2013;133(4):551-559.

82. Kanchanabat B, Stapanavatr W, Meknavin S, Soorapanth C, Sumanasrethakul C, Kanchanasuttirak P. Systematic review and meta-analysis on the rate of postoperative venous 
thromboembolism in orthopaedic surgery in Asian patients without thromboprophylaxis. British Journal of Surgery. 2011;98(10):1356-1364.

83. Jaffer AK, Barsoum WK, Krebs V, Hurbanek JG, Morra N, Brotman DJ. Duration of Anesthesia and Venous Thromboembolism After Hip and Knee Arthroplasty. Mayo Clinic Proceedings. 2005;80(6):732-738.

84. Niki Y, Matsumoto H, Hakozaki A, Mochizuki T, Momohara S. Rheumatoid arthritis: A risk factor for deep venous thrombosis after total knee arthroplasty? Comparative study with osteoarthritis. Journal of Orthopaedic Science. 2010;15(1):57-63.

85. Sadeghi B, Romano PS, Maynard G, et al. Mechanical and suboptimal pharmacologic prophylaxis and delayed mobilization but not morbid obesity are associated with venous thromboembolism after total knee arthroplasty: A case-control study. Journal of Hospital Medicine. 2012.

86. Chandrasekaran S, Ariaretnam SK, Tsung J, Dickison D. Early mobilization after total knee replacement reduces the incidence of deep venous thrombosis. ANZ Journal of Surgery. 2009;79(7-8):526-529.

87. White RH, Gettner S, Newman JM, Trauner KB, Romano PS. Predictors of rehospitalization for symptomatic venous thromboembolism after total hip arthroplasty. New England Journal of Medicine. 2000;343(24):1758-1764.

88. Barrett J, Baron JA, Losina E, Wright J, Mahomed NN, Katz JN. Bilateral total knee replacement: Staging and pulmonary embolism. Journal of Bone and Joint Surgery - Series A. 2006;88(10):2146-2151.

89. Venous Thromboembolism: reducing the risk of venous thromboembolism (deep vein thrombosis and pulmonary embolism) in patients admitted to hospital. 2 ed. London: National Collaborating Centre for Acute Care, National Clinical Guidance Centre - Acute and Chronic Conditions; 2010.

90. Rosencher N, Noack H, Feuring M, Clemens A, Friedman RJ, Eriksson BI. Type of anaesthesia and the safety and efficacy of thromboprophylaxis with enoxaparin or dabigatran etexilate in major orthopaedic surgery: Pooled analysis of three randomized controlled trials. Thrombosis Journal. 2012;10.

91. Mortazavi SMJ, Hansen P, Zmistowski B, Kane PW, Restrepo C, Parvizi J. Hematoma Following Primary Total Hip Arthroplasty: A Grave Complication. Journal of Arthroplasty. 2013;28(3):498-503.

92. Papakostidis C, Harwood PJ, Karadimas EJ, Tan HB, Giannoudis PV. The timing of drug administration for thromboprophylaxis following orthopaedic surgery: Evidence and controversies related to treatment initiation and duration. Current Vascular Pharmacology. 2011;9(1):11-18.

93. Kakkar AK, Brenner B, Dahl OE, et al. Extended duration rivaroxaban versus short-term enoxaparin for the prevention of venous thromboembolism after total hip arthroplasty: a double-blind, randomised controlled trial. The Lancet. 2008;372(9632):31-39.

94. Hozumi T, Eisenberg M, Sugioka K, et al. Change in coronary flow reserve on transthoracic Doppler echocardiography after a single high-fat meal in young healthy men. Annals of Internal Medicine. 2002;136(7):523-528.

95. Varraso R, Kabrhel C, Goldhaber SZ, Rimm EB, Camargo Jr CA. Prospective study of diet and venous thromboembolism in US women and men. American Journal of Epidemiology. 2012;175(2):114-126.

96. Maccallum PK, Ashby D, Hennessy EM, et al. Cumulative flying time and risk of venous thromboembolism. British Journal of Haematology. 2011;155(5):613-619.

97. Willenberg $\mathrm{T}$, Schumacher A, Amann-Vesti B, et al. Impact of obesity on venous hemodynamics of the lower limbs. Journal of Vascular Surgery. 2010;52(3):664-668.

98. Willenberg T, Clemens R, Haegeli LM, Amann-Vesti B, Baumgartner I, Husmann M. The influence of abdominal pressure on lower extremity venous pressure and hemodynamics: A human in-vivo model simulating the effect of abdominal obesity. European Journal of Vascular and Endovascular Surgery. 2011;41(6):849-855.

99. Stein PD, Beemath A, Olson RE. Obesity as a risk factor in venous thromboembolism. American Journal of Medicine. 2005;118(9):978-980. 
100. Pearse EO, Caldwell BF, Lockwood RJ, Hollard J. Early mobilisation after conventional knee replacement may reduce the risk of post-operative venous thromboembolism. Journal of Bone and Joint Surgery - Series B. 2007;89(3):316-322.

101. Steffen LM, Folsom AR, Cushman M, Jacobs Jr DR, Rosamond WD. Greater fish, fruit, and vegetable intakes are related to lower incidence of venous thromboembolism: The Longitudinal Investigation of Thromboembolism Etiology. Circulation. 2007;115(2):188-195.

102. Janssen $H$, Trevino C, Williams D. Hemodynamic alterations in venous blood flow produced by external pneumatic compression. Journal of Cardiovascular Surgery. 1993;34:441-447.

103. Bergan JJ. The Vein Book: Academic Press; 2006.

104. Kibbe MR, Pearce WH, Yao JST. Modern Trends in Vascular Surgery: Venous Disorders: McGraw-Hill Companies; 2010.

105. Image found on Phlebology Journal website. however no source could be identified.; http://phleb.rsmjournals.com/content/27/5/219/F1.expansion.html.

106. Bayer Pharma. Mechanical Devices. http://www.thrombosisadviser.com/en/currenttreatments/mechanical-devices/index.php. Accessed 09/01/13.

107. Best AJ, Williams S, Crozier A, Bhatt R, Gregg PJ, Hui ACW. Graded compression stockings in elective orthopaedic surgery. An assessment of the in vivo performance of commercially available stockings in patients having hip and knee arthroplasty. Journal of Bone and Joint Surgery - Series B. 2000;82(1):116-118.

108. Geerts WH, Pineo GF, Heit JA, et al. Prevention of venous thromboembolism: The Seventh ACCP Conference on Antithrombotic and Thrombolytic Therapy. Chest. 2004;126(3 SUPPL.):338S-400S.

109. Macatangay C, Todd SR, Tyroch AH. Thromboembolic prophylaxis with intermittent pneumatic compression devices in trauma patients: a false sense of security? Journal of trauma nursing : the official journal of the Society of Trauma Nurses. 2008;15(1):12-15.

110. Hardwick ME, Pulido PA, Colwell Jr CW. A mobile compression device compared with lowmolecular-weight heparin for prevention of venous thromboembolism in total hip arthroplasty. Orthopaedic Nursing. 2011;30(5):312-316.

111. Tsukano Y, Sakanashi Y, Takeshita J. Compression characteristics of graduated compression stockings used for prevention of venous thromboembolism (Abstract only). Japanese Journal of Anesthesiology. 2007;56(11):1347-1352.

112. Kakkos SK, Warwick D, Nicolaides AN, Stansby GP, Tsolakis IA. Combined (mechanical and pharmacological) modalities for the prevention of venous thromboembolism in joint replacement surgery. Journal of Bone and Joint Surgery - Series B. 2012;94 B(6):729-734.

113. Pour AE, Keshavarzi NR, Purtill JJ, Sharkey PF, Parvizi J. Is Venous Foot Pump Effective In Prevention of Thromboembolic Disease After Joint Arthroplasty: A Meta-Analysis. Journal of Arthroplasty. 2012.

114. Lippi G, Favaloro EJ, Cervellin G. Prevention of venous thromboembolism: Focus on mechanical prophylaxis. Seminars in Thrombosis and Hemostasis. 2011;37(3):237-251.

115. Bunescu A, Widman J, Lenkei R, Menyes P, Levin K, Egberg N. Increases in circulating levels of monocyte-platelet and neutrophil-platelet complexes following hip arthroplasty. Clinical Science. 2002;102(3):279-286.

116. Becker RC. Aspirin and the Prevention of Venous Thromboembolism New England Journal of Medicine. 2012;366(21):2028-2030.

117. Tousoulis D, Paroutoglou IP, Papageorgiou N, Charakida M. Recent therapeutic approaches to platelet activation in coronary artery disease. Pharmacology \& Therapeutics. 2010;127(2):108-120.

118. Bayer Pharma. Clot Formation. 2012; http://www.thrombosisadviser.com/en/image.php?image=clot-formation-coagcascade\&category= coagulationcascade.

119. The Australian \& New Zealand Working Party on the Management and Prevention of Venous Thromboembolism. Prevention of venous thromboembolism: Best practice guidelines for Australia and New Zealand. 2008.

120. Stirling Y. Warfarin-induced changes in procoagulant and anticoagulant proteins. Blood Coagulation and Fibrinolysis. 1995;6(5):361-373. 
121. Molnar RB, Jenkin DE, Millar MJ, Campbell D, Harris IA. The australian arthroplasty thromboprophylaxis survey. Journal of Arthroplasty. 2012;27(2):173-179.

122. Kearon C. Duration of Venous Thromboembolism Prophylaxis after Surgery. Chest. 2003;124(6 SUPPL.):386S-392S.

123. Fisher WD. Impact of venous thromboembolism on clinical management and therapy after hip and knee arthroplasty (Abstract only). Canadian Journal of Surgery. 2011;54(5):344-351.

124. Bergqvist D, Arcelus JI, Felicissimo P. Post-discharge compliance to venous thromboembolism prophylaxis in high-risk orthopaedic surgery, results from the ETHOS registry (Abstract only). Thrombosis and Haemostasis. 2012;107(2):280-287.

125. Fanikos J, Stevens LA, Labreche $M$, et al. Adherence to Pharmacological Thromboprophylaxis Orders in Hospitalized Patients (Abstract only). American Journal of Medicine. 2010;123(6):536-541.

126. Turpie AG, Lassen MR, Davidson BL, et al. Rivaroxaban versus enoxaparin for thromboprophylaxis after total knee arthroplasty (RECORD4): a randomised trial. The Lancet. 2009;373(9676):1673-1680.

127. Cao YB, Zhang JD, Shen H, Jiang YY. Rivaroxaban versus enoxaparin for thromboprophylaxis after total hip or knee arthroplasty: A meta-analysis of randomized controlled trials. European Journal of Clinical Pharmacology. 2010;66(11):1099-1108.

128. Gómez-Outes A, Terleira-Fernández AI, Suárez-Gea ML, Vargas-Castrillón E. Dabigatran, rivaroxaban, or apixaban versus enoxaparin for thromboprophylaxis after total hip or knee replacement: Systematic review, meta-analysis, and indirect treatment comparisons. BMJ (Online). 2012;344(7863).

129. Nieto JA, Espada NG, Merino RG, González TC. Dabigatran, Rivaroxaban and Apixaban versus Enoxaparin for thomboprophylaxis after total knee or hip arthroplasty: Pool-analysis of phase III randomized clinical trials. Thrombosis Research. 2012;130(2):183-191.

130. Neumann I, Rada G, Claro JC, et al. Oral direct factor Xa inhibitors versus low-molecularweight heparin to prevent venous thromboembolism in patients undergoing total hip or knee replacement a systematic review and meta-analysis. Annals of Internal Medicine. 2012;156(10):710-719.

131. Makris M, Van Veen JJ, Tait CR, Mumford AD, Laffan M. Guideline on the management of bleeding in patients on antithrombotic agents. British Journal of Haematology. 2013;160(1):35-46.

132. Chahal GS, Saithna A, Brewster M, et al. A comparison of complications requiring return to theatre in hip and knee arthroplasty patients taking enoxaparin versus rivaroxaban for thromboprophylaxis. Ortopedia Traumatologia Rehabilitacja. 2013;15(2):125-129.

133. Miyares MA, Davis K. Newer oral anticoagulants: A review of laboratory monitoring options and reversal agents in the hemorrhagic patient. American Journal of Health-System Pharmacy. 2012;69(17):1473-1484.

134. Levi MM, Eerenberg E, Löwenberg E, Kamphuisen PW. Bleeding in patients using new anticoagulants or antiplatelet agents: risk factors and management. Netherlands Journal of Medicine. 2010;68(2):68-76.

135. Keays AC, Mason M, Keays SL, Newcombe PA. The effect of anticoagulation on the restoration of range of motion after total knee arthroplasty: Enoxaparin versus aspirin. Journal of Arthroplasty. 2003;18(2):180-185.

136. Bek D, Besksaç B, Della Valle AG, Sculco TP, Salvati EA. Aspirin Decreases the Prevalence and Severity of Heterotopic Ossification After 1-Stage Bilateral Total Hip Arthroplasty. Journal of Arthroplasty. 2009;24(2):226-232.

137. AMH. Australian Medicines Handbook. Adelaide2012.

138. Blann AD, Lip GYH, Turpie AG. Thrombosis in Clinical Practice. Vol 1. London: Taylor \& Francis Group; 2005.

139. Campbell D, Smith $\mathrm{P}$, Lewis $\mathrm{P}$, Bruce W. Controversies of thrombophylaxis following knee arthroplasty surgery. ANZ Journal of Surgery. 2010;80(6):391-395.

140. Evensen AE, Sanson-Fisher R, D'Este C, Fitzgerald M. Trends in publications regarding evidence-practice gaps: A literature review. Implementation Science. 2010;5(1). 
141. Wheatley I. Professional Autonomy of Victorian Surgeons in the Context of Law and Ethics, Deakin University; 2012.

142. Ragucci MV, Leali A, Moroz A, Fetto J. Comprehensive deep venous thrombosis prevention strategy after total-knee arthroplasty. American Journal of Physical Medicine and Rehabilitation. 2003;82(3):164-168.

143. Lachiewicz PF, Soileau ES. Mechanical calf compression and aspirin prophylaxis for total knee arthroplasty. Clinical Orthopaedics and Related Research. 2007(464):61-64.

144. Westrich GH, Haas SB, Mosca P, Peterson M. Meta-analysis of thromboembolic prophylaxis after total knee arthroplasty. Journal of Bone and Joint Surgery - Series B. 2000;82(6):795800.

145. Lotke PA, Lonner JH. The benefit of aspirin chemoprophylaxis for thromboembolism after total knee arthroplasty. Clinical Orthopaedics and Related Research. 2006(452):175-180.

146. Morris JK, Fincham BM. Intermittent pneumatic compression for venous thromboembolism prophylaxis in total knee arthroplasty. Orthopaedics. 2012;35(12):e1716-1721.

147. Barrack RL. Current guidelines for total joint VTE prophylaxis: Dawn of a new day. Journal of Bone and Joint Surgery - Series B. 2012;94 B(11 SUPPL.A):3-7.

148. Dorr LD, Gendelman V, Maheshwari AV, Boutary M, Wan Z, Long WT. Multimodal thromboprophylaxis for total hip and knee arthroplasty based on risk assessment. Journal of Bone and Joint Surgery - Series A. 2007;89 A(12):2648-2657.

149. Berend KR, Lombardi Jr AV. Multimodal venous thromboembolic disease prevention for patients undergoing primary or revision total joint arthroplasty: the role of aspirin. American journal of orthopedics (Belle Mead, N.J.). 2006;35(1):24-29.

150. Della Valle AG, Serota A, Go G, et al. Venous thromboembolism is rare with a multimodal prophylaxis protocol after total hip arthroplasty. Clinical Orthopaedics and Related Research. 2006(444):146-153.

151. Patel AR, Crist MK, Nemitz J, Mayerson JL. Aspirin and Compression Devices versus LowMolecular-Weight Heparin and PCD for VTE Prophylaxis in Orthopedic Oncology Patients. Journal of Surgical Oncology. 2010;102:276-281.

152. Jameson SS, Charman SC, Gregg PJ, Reed MR, Van Der Meulen JH. The effect of aspirin and low-molecular-weight heparin on venous thromboembolism after hip replacement: A non-randomised comparison from information in the National Joint Registry. Journal of Bone and Joint Surgery - Series B. 2011;93 B(11):1465-1470.

153. Bozic KJ, Vail TP, Pekow PS, Maselli JH, Lindenauer PK, Auerbach AD. Does aspirin have a role in venous thromboembolism prophylaxis in total knee arthroplasty patients? Journal of Arthroplasty. 2010;25(7):1053-1060.

154. Gesell MW, González Della Valle A, Bartolomé García S, et al. Safety and Efficacy of Multimodal Thromboprophylaxis Following Total Knee Arthroplasty. A Comparative Study of Preferential Aspirin vs. Routine Coumadin Chemoprophylaxis. Journal of Arthroplasty. 2012.

155. Woller SC, Bertin KC, Stevens SM, et al. A Prospective Comparison of Warfarin to Aspirin for Thromboprophylaxis in Total Hip and Total Knee Arthroplasty. Journal of Arthroplasty. 2012;27(1):1-9.e2.

156. AAOS. Clinical Guideline on Prevention of Pulmonary Embolism in Patients Undergoing Total Hip or Knee Arthroplasty. 1st ed. Rosemont: American Academy of Orthopaedic Surgeons; 2007.

157. Rodgers A, MacMahon S, Collins R, Prentice C. Prevention of pulmonary embolism and deep vein thrombosis with low dose aspirin: Pulmonary Embolism Prevention (PEP) trial. Lancet. 2000;355(9212):1295-1302.

158. MacMahon S, Rodgers A, Collins R, Farrell B. Antiplatelet therapy to prevent thrombosis after hip fracture: rationale for a randomised trial. Journal of Bone and Joint Surgery - Series B. 1994;76:521-524.

159. Cohen A, Quinlan D, Cimminiello $\mathrm{C}$, et al. PEP trial (multiple letters). Lancet. 2000;356(9225):247-251. 
160. Smith EB, Parvizi J, Purtill JJ. Delayed surgery for patients with femur and hip fractures Risk of deep venous thrombosis. Journal of Trauma - Injury, Infection and Critical Care. 2011;70(6):E113-E116.

161. Zahn HR, Skinner JA, Porteous MJ. The preoperative prevalence of deep vein thrombosis in patients with femoral neck fractures and delayed operation. Injury. 1999;30(9):605-607.

162. Mangat KS, Mehra A, Yunas I, Porter K. Venous thromboprophylaxis in trauma: A review. Trauma. 2006;8(4):233-247.

163. Jacoby RC, Owings JT, Holmes J, Battistella FD, Gosselin RC, Paglieroni TG. Platelet activation and function after trauma. Journal of Trauma - Injury, Infection and Critical Care. 2001;51(4):639-647.

164. Geerts WH, Bergqvist D, Pineo GF, et al. Prevention of venous thromboembolism: American College of Chest Physicians evidence-based clinical practice guidelines (8th edition). Chest. 2008;133(6 SUPPL. 6):381S-453S.

165. Prevention of Venous Thromboembolism. 5th Edition ed: The Australia and New Zealand Working Party on the Mangement and Prevention of Venous Thromboembolism; 2010.

166. AAOS. Preventing Venous Thromboembolic Disease in Patients Undergoing Elective Hip and Knee Arthroplasty - Evidence BAsed Guideline and Evidence Report 2011. http://www.aaos.org/research/guidelines/VTE/VTE_full_guideline.pdf. Accessed 09/04/2012.

167. Guidelines for VTE prophylaxis for hip and knee arthroplasty: Arthroplasty Society of Australia; 2010.

168. Learhinan ER, Alderman CP. Venous Thromboembolism Prophylaxis in a South Australian Teaching Hospital. Hematology. 2003;37:1398-1402.

169. Sobieraj-Teague DM, Eiketboom JW, Hirsch A. How can we reduce disagreement among guidelines for venous thromboembolism prevention? Journal of Thrombosis and Haemostasis. 2010;8:675-677.

170. Lachiewicz PF. AAOS Guidelines for the Prevention of Symptomatic Pulmonary Embolism after Total Hip and Total Knee Arthroplasty: Advantages and Disadvantages. Seminars in Arthroplasty. 2009;20(4):230-234.

171. Struijk-Mulder MC, Ettema HB, Verheyen CC, B $\tilde{A}^{1 / 4} / 1$ er HR. Comparing consensus guidelines on thromboprophylaxis in orthopedic surgery. Journal of Thrombosis and Haemostasis. 2010;8(4):678-683.

172. Arcelus JI, Felicissimo P. Venous thromboprophylaxis duration and adherence to international guidelines in patients undergoing major orthopaedic surgery: Results of the international, longitudinal, observational DEIMOS registry. Thrombosis Research. 2013;131(6):e240-e246.

173. National Institute of Clinical Studies. Evidence-Practice Gaps. Vol 1. Melbourne 2003.

174. Phillips NM, Heazlewood VJ. Venous thromboembolism prophylaxis audit in two Queensland hospitals. Internal Medicine Journal. 2013;43(5):560-566.

175. Cabana MD, Rand CS, Powe NR, et al. Why don't physicians follow clinical practice guidelines?: A framework for improvement. Journal of the American Medical Association. 1999;282(15):1458-1465.

176. Feder G, Eccles M, Grol R, Griffiths C, Grimshaw J. Clinical guidelines: Using clinical guidelines. British Medical Journal. 1999;318(7185):728-730.

177. Duff J, Walker K, Omari A. Translating Venous Thromboembolism (VTE) Prevention Evidence into Practice: A Multidisciplinary Evidence Implementation Project. Worldviews on Evidence-Based Nursing. 2011;8(1):30-39.

178. Straus S, Tetroe J, Graham ID. Knowledge Translation in Health Care: Moving from Evidence to Practice. Chichester: Blackwell Publishing Ltd.; 2009.

179. Duff J, Walker K, Omari A, Stratton C. Prevention of venous thromboembolism in hospitalized patients: Analysis of reduced cost and improved clinical outcomes. Journal of Vascular Nursing. 2013;31(1):9-14.

180. Sharif KM, Rehman SF, Nunn T, Bagga TK. Implementation of the NICE guidelines for venous thromboprophylaxis; A national survey of hip surgeons (Abstract only). HIP International. 2009;19(1):58-63. 
181. Rodgers A, Gray H, MacMahon S. Pharmacological thromboprophylaxis in hip and knee surgery: A survey of New Zealand orthopaedic surgeons. Australian and New Zealand Journal of Surgery. 1994;64(3):167-172.

182. Walker N, Rodgers A, Gray H. Changing patterns of pharmacological thromboprophylaxis use by orthopaedic surgeons in New Zealand. ANZ Journal of Surgery. 2002;72(5):335-338.

183. Zairul-Nizam ZF, Gul YA. Malaysian orthopaedic surgeons' approach to venous thromboembolic disease prophylaxis: attitudes and practice. Journal of orthopaedic surgery (Hong Kong). 2003;11(2):178-183.

184. White RH, Romano PS, Zhou H, Rodrigo J, Bargar W. Incidence and time course of thromboembolic outcomes following total hip or knee arthroplasty. Archives of Internal Medicine. 1998;158(14):1525-1531.

185. Spyropoulos AC, Hussein M, Lin J, Battleman D. Rates of symptomatic venous thromboembolism in US surgical patients: A retrospective administrative database study. Journal of Thrombosis and Thrombolysis. 2009;28(4):458-464.

186. Dahl OE. Continuing out-of-hospital prophylaxis following major orthopaedic surgery: What now? Haemostasis. 2000;30(SUPPL. 2):101-105.

187. eTG. Antibiotic. 13 ed: Therapeutic Guidelines Limited; 2010: www.tg.org.au. Accessed 22/12/10.

188. Aynardi M, Brown PB, Post Z, Orozco F, Ong A. Warfarin for Thromboprophylaxis Following Total Joint Arthroplasty. Are Patients Safely Anti-Coagulated? Journal of Arthroplasty. 2013.

189. Mantilla CB, Horlocker TT, Schroeder DR, Berry DJ, Brown DL. Risk factors for clinically relevant pulmonary embolism and deep venous thrombosis in patients undergoing primary hip or knee arthroplasty. Anesthesiology. 2003;99(3):552-560.

190. Zhang ZJ, Zhao XY, Kang Y, et al. The influence of body mass index on life quality and clinical improvement after total hip arthroplasty. Journal of Orthopaedic Science. 2012;17(3):219-225.

191. Thorpe C, Ryan B, McLean SL, et al. How to obtain excellent response rates when surveying physicians. Family Practice. 2009;26(1):65-68.

192. Levy RM, Shapiro M, Halpern SD, Ming ME. Effect of personalization and candy incentive on response rates for a mailed survey of dermatologists. Journal of Investigative Dermatology. 2012;132(3 PART 1):724-726.

193. Braithwaite D, Emery J, de Lusignan S, Sutton S. Using the internet to conduct surveys of health professionals: A valid alternative? Family Practice. 2003;20(5):545-551.

194. Kaplowitz MD, Hadlock TD, Levine R. A comparison of web and mail survey response rates. Public Opinion Quarterly. 2004;68(1):94-101.

195. Li F, Walker K, McInnes E, Duff J. Testing the effect of a targeted intervention on nurses' compliance with " best practice" mechanical venous thromboembolism prevention. Journal of Vascular Nursing. 2010;28(3):92-96.

196. Timmermans S. From autonomy to accountability: the role of clinical practice guidelines in professional power. Perspectives in biology and medicine. 2005;48(4):490-501.

197. Rogers BA, Phillips S, Foote J, Drabu KJ. Is there adequate provision of venous thromboembolism prophylaxis following hip arthroplasty? An audit and international survey. Annals of the Royal College of Surgeons of England. 2010;92(8):668-672.

198. Thrombosis Guidelines Provide Weak Recommendation of Aspirin. Heartwire CME. 2012. http://www.medscape.org/viewarticle/759145.

199. Cummings SM, Savitz LA, Konrad TR. Reported response rates to mailed physician questionnaires. Health Services Research. 2001;35(6):1347-1355.

200. Bhandari M, Devereaux PJ, Swiontkowski MF, et al. A randomized trial of opinion leader endorsement in a survey of orthopaedic surgeons: Effect on primary response rates. International Journal of Epidemiology. 2003;32(4):634-636.

201. Groves RM, Peytcheva E. The impact of nonresponse rates on nonresponse bias: A metaanalysis. Public Opinion Quarterly. 2008;72(2):167-189.

202. Livingston EH, Wislar JS. Minimum response rates for survey research. Archives of Surgery. 2012;147(2):110. 
203. Hansen EC. Successful Qualitative Health Research: A practical introduction. Sydney: Allen \& Unwin; 2006.

204. Kennedy TJT, Lingard LA. Making sense of grounded theory in medical education. Medical Education. 2006;40(2):101-108.

205. Tan TP, Stokes T, Shaw EJ. Use of qualitative research as evidence in the clinical guideline program of the National Institute for Health and Clinical Excellence. International Journal of Evidence-Based Healthcare. 2009;7(3):169-172.

206. Mirkazemi C, Bereznicki LR, Peterson GM. Are the national orthopaedic thromboprophylaxis guidelines appropriate? ANZ Journal of Surgery. 2012;82(12):913-917.

207. Heartwire. Medscape Education: Thrombosis Guidelines Provide Weak Recommendation of Aspirin. 2012. Accessed 24 May, 2012.

208. Turner T, Misso M, Harris C, Green S. Development of evidence-based clinical practice guidelines (CPGs): Comparing approaches. Implementation Science. 2008;3(1).

209. Anderson DR, Dunbar MJ, Bohm ER, et al. Aspirin versus low-molecular-weight heparin for extended venous thromboembolism prophylaxis after total hip arthroplasty: A randomized trial. Annals of Internal Medicine. 2013;158(11):800-806.

210. Cook JV, Dickinson HO, Eccles MP. Response rates in postal surveys of healthcare professionals between 1996 and 2005: An observational study. BMC Health Services Research. 2009;9.

211. Prasad V, Vandross A, Toomey C, et al. A Decade of Reversal: An Analysis of 146 Contradicted Medical Practices. Mayo Clinic Proceedings. 2013.

212. Malek LA, Reynolds HR, Forman SA, et al. Late coronary intervention for totally occluded left anterior descending coronary arteries in stable patients after myocardial infarction: Results from the Occluded Artery Trial (OAT). American Heart Journal. 2009;157(4):724732.

213. Moscucci M. Medical reversal, clinical trials, and the "late" open artery hypothesis in acute myocardial infarction. Archives of Internal Medicine. 2011;171(18):1643-1644.

214. Jhaveri RR, Reynolds HR, Katz SD, et al. Heart failure in post-MI patients with persistent IRA occlusion: Prevalence, risk factors, and the long-term effect of PCI in the Occluded Artery Trial (OAT). Journal of Cardiac Failure. 2012;18(11):813-821.

215. Hastings RS, Hochman JS, Dzavik V, et al. Effect of late revascularization of a totally occluded coronary artery after myocardial infarction on mortality rates in patients with renal impairment. American Journal of Cardiology. 2012;110(7):954-960.

216. Prasad V, Gall V, Cifu A. The frequency of medical reversal. Archives of Internal Medicine. 2011;171(18):1675-1676.

217. Fatovich DM. Medical reversal: What are you doing wrong for your patient today? Emergency Medicine Australia. 2013;25:1-3.

218. Guyatt GH, Cook DJ, Jaeschke R, Pauker SG, Schünemann HJ. Grades of recommendation for antithrombotic agents: American College of Chest Physicians evidence-based clinical practice guidelines (8th edition). Chest. 2008;133(6 SUPPL. 6):123S-131S. 